\title{
Driver Speed Behaviour on Freeway Deceleration Speed-Change Lanes
}

\author{
Submitted By \\ Ramy H. Soliman El-Basha, B. Eng.
}

A thesis submitted to the faculty of Graduate Studies and Research in partial fulfillment of the requirements for the degree of Master of Applied Science

\author{
Department of Civil and Environmental Engineering \\ Carleton University \\ Ottawa, Ontario, Canada
}

(C) Ramy H. Soliman El-Basha 2006

The Master of Applied Science in Civil Engineering Program is a joint program with the University of Ottawa, administered by the Ottawa-Carleton Institute of Civil Engineering 


$\begin{array}{ll}\begin{array}{l}\text { Library and } \\ \text { Archives Canada }\end{array} & \begin{array}{l}\text { Bibliothèque et } \\ \text { Archives Canada }\end{array} \\ \begin{array}{l}\text { Published Heritage } \\ \text { Branch }\end{array} & \begin{array}{l}\text { Direction du } \\ \text { Patrimoine de l'édition }\end{array} \\ \begin{array}{l}\text { 395 Wellington Street } \\ \text { Ottawa ON K1A ON4 }\end{array} & \begin{array}{l}\text { 395, rue Wellington } \\ \text { Ottawa ON K1A ON4 } \\ \text { Canada }\end{array}\end{array}$

Your file Votre référence ISBN: 978-0-494-16467-9 Our file Notre référence ISBN: 978-0-494-16467-9

NOTICE:

The author has granted a nonexclusive license allowing Library and Archives Canada to reproduce, publish, archive, preserve, conserve, communicate to the public by telecommunication or on the Internet, loan, distribute and sell theses worldwide, for commercial or noncommercial purposes, in microform, paper, electronic and/or any other formats.

The author retains copyright ownership and moral rights in this thesis. Neither the thesis nor substantial extracts from it may be printed or otherwise reproduced without the author's permission.
AVIS:

L'auteur a accordé une licence non exclusive permettant à la Bibliothèque et Archives Canada de reproduire, publier, archiver, sauvegarder, conserver, transmettre au public par télécommunication ou par l'Internet, prêter, distribuer et vendre des thèses partout dans le monde, à des fins commerciales ou autres, sur support microforme, papier, électronique et/ou autres formats.

L'auteur conserve la propriété du droit d'auteur et des droits moraux qui protège cette thèse. $\mathrm{Ni}$ la thèse ni des extraits substantiels de celle-ci ne doivent être imprimés ou autrement reproduits sans son autorisation.
In compliance with the Canadian

Privacy Act some supporting forms may have been removed from this thesis.

While these forms may be included in the document page count, their removal does not represent any loss of content from the thesis.
Conformément à la loi canadienne sur la protection de la vie privée, quelques formulaires secondaires ont été enlevés de cette thèse.

Bien que ces formulaires aient inclus dans la pagination, il n'y aura aucun contenu manquant.

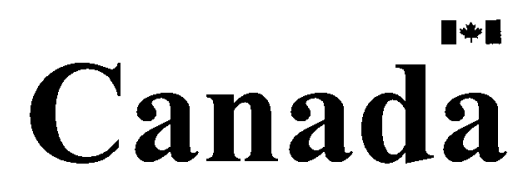




\begin{abstract}
Deceleration speed-change lanes (SCLs) and exit ramp terminals on freeways are typically designed to allow vehicles to depart off the freeway in an organized and efficient driving manner. Freeway diverge areas with adequate SCL lengths should enable the exiting drivers to diverge off the freeway through traffic, decelerate to the desired ramp speed, and exit the freeway safely and comfortably. Safety problems can be expected if drivers are forced to reduce speed on the main traffic lanes or to decelerate at a very high rate. In Canada and the US, the current design guides suggest that operation and safety could be enhanced on freeway SCLs by increasing their lengths. In this study, the driver behaviour at freeway diverge areas was examined using data collected on thirteen exit ramp terminals on Highway 417 in the City of Ottawa, Canada. The speed, geometric, and traffic data were collected and employed to model $85^{\text {th }}$ percentile speeds and deceleration rates using linear regression analysis. The modelling attempts integrated in this study resulted in 34 statistically significant predictive models at $5 \%$ level of significance. Design charts were developed for easy use by highway professionals.
\end{abstract}




\section{ACKNOWLEDGEMENTS}

First, I would like to truthfully thank God (Allah) for helping me accomplish this research. Without him, this would not be possible.

Second, I would like to express my sincere gratitude and appreciation to my thesis supervisors, Professor Yasser Hassan and Professor Tarek A. Sayed, for their profound patience and encouragement during my study period. In addition, I would like to genuinely express my enormous respect to my supervisors in supporting me until the end for the fulfillment of this research. The endless support and gracious guidance of Professor Yasser Hassan helped me to reach to the highest levels of confidence.

Third, the financial support by the Canadian Foundation for Innovation (CFI), the Ontario Innovation Trust (OIT), and the National Sciences and Engineering Research Council of Canada (NSERC) for equipment used in speed data collection is acknowledged. Further, the research assistance received from the NSERC Operating Grant of Professor Tarek A. Sayed (University of British Columbia) is appreciatively and enthusiastically acknowledged.

Fourth, I would like to pay thanks and regards to my colleague researcher Eng. Alauddin M. Ahammed for his cooperation in the speed data collection. Special thanks also go to Eng. Mohamed E. A. Sarhan, Mrs. Allison Giest (Ministry of Transportation Ontario), and Mr. Mike Gallagher (Morrison Hersfield) for providing the necessary materials and data that were used for the completion of this study. Additionally, I would also like to express my regards to Mr. Dick Wells and Mr. Wayne Millet (OttawaCarleton School Board), Mr. Tom Carmody (City of Ottawa), Mr. Eric Booth (Ontario 
Provincial Police), and the Ottawa Police for their cooperation during the speed data collection phase of this study.

Finally, I would like to dedicate this thesis to my dearest father (Eng. Hussein ElBasha), beloved mother (Mrs. Nadia Mustafa), and other family members for their moral and financial support for the past six years. 


\section{TABLE OF CONTENTS}

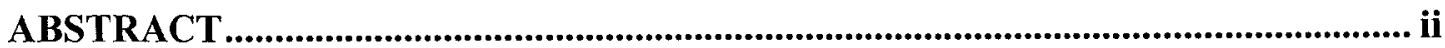

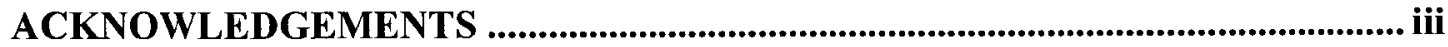

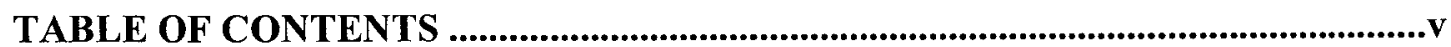

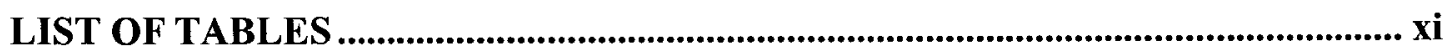

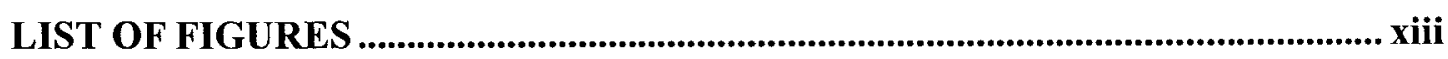

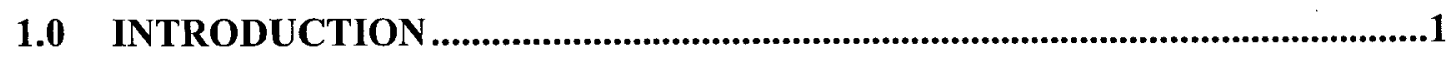

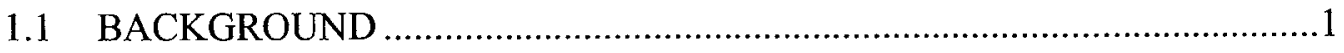

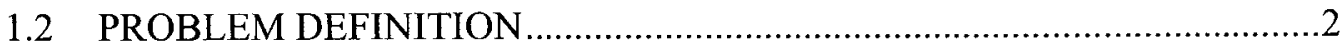

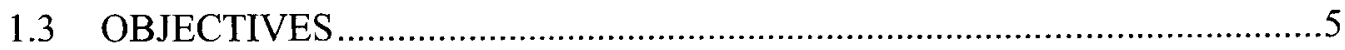

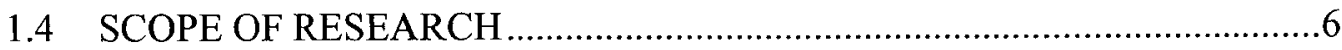

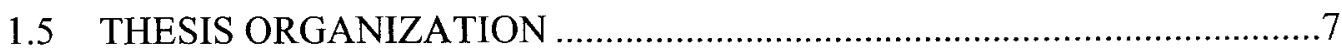

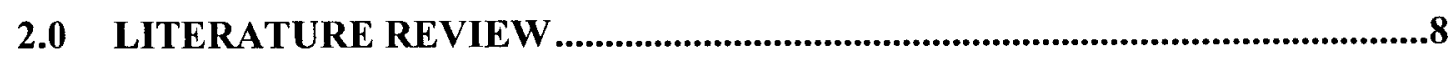

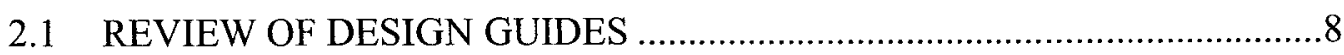

2.1.1 Design Criteria and Interchange Configurations ...........................

2.1.2 Freeway Diverge Areas.........................................................11

2.1.3 Interchange Ramp Terminals ................................................12

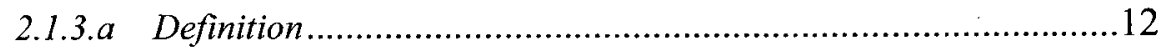

2.1.3.b Design Consistency of Ramps ................................................15

2.1.3.c Ramp Width and Speed ......................................................17

2.1.3.d Gore Area and Physical Nose ...............................................18

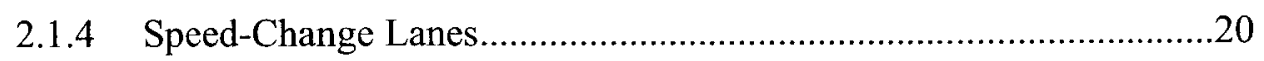

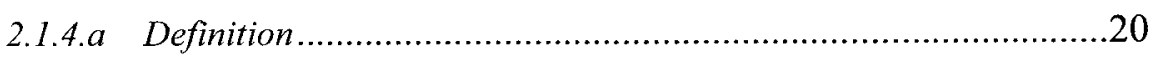

$\mathrm{V}$ 
2.2 REVIEW OF PREVIOUS RESEARCH …......................................27

2.2.1 Driving Characteristics on Freeway Diverge Areas .......................27

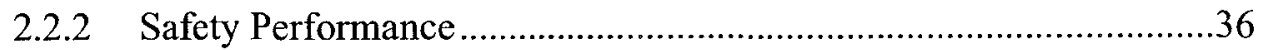

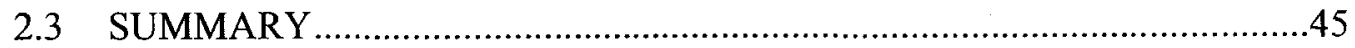

3.0 DATA COLLECTION AND PREPARATION ..............................................46

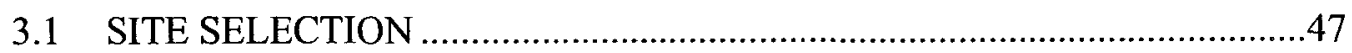

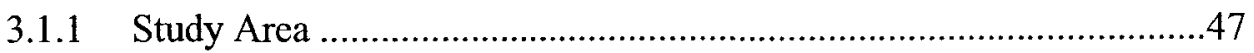

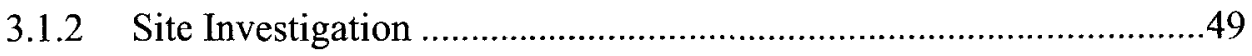

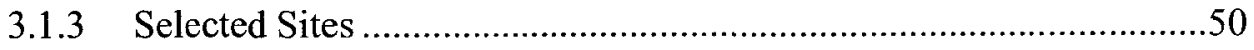

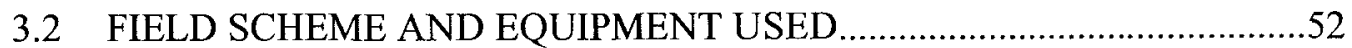

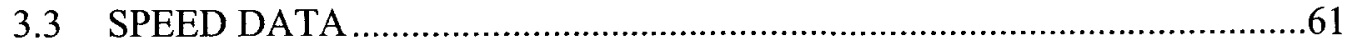

3.3.1 Speed Data Retrieval..............................................................61

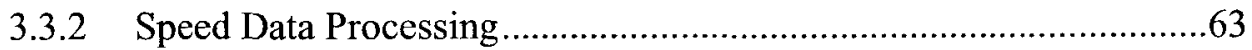

3.3.2.a Correction for Vertical and Horizontal Offsets ..........................64

3.3.2.b Corrected Distance and Speed Calculations ............................68

3.3.2.c Deceleration Calculations and Adjustment of Speed Profiles ........68

3.3.3 Preparation of Aggregated Driver Behaviour Measures...................72

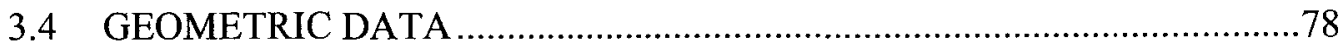

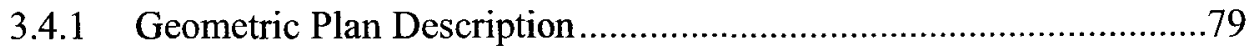

3.4.2 Measurement of Geometric Features ............................................. 81

3.4.2.a Length of Speed-Change Lane ................................................ 81

3.4.2.b Transition Length ........................................................... 81

vi 


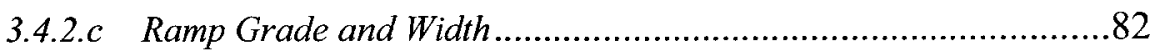

3.4.2.d Number of Basic Freeway Lanes......................................... 82

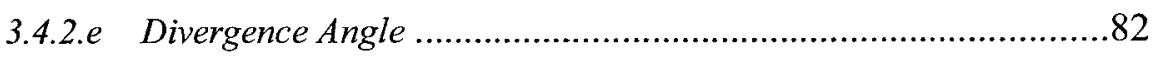

3.4.2.f Lengths of Freeway Segments............................................ 82

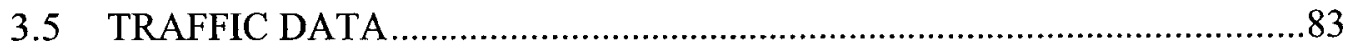

3.5.1 Equivalent Hourly Traffic Volumes ..........................................83

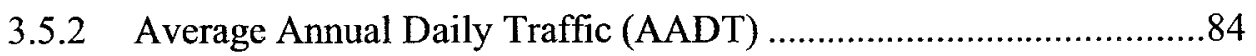

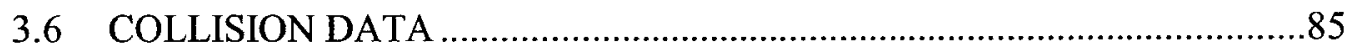

3.6.1 Collision Data Source and Preparation ......................................85

3.6.2 Analysis Parameter for Safety Performance ................................86

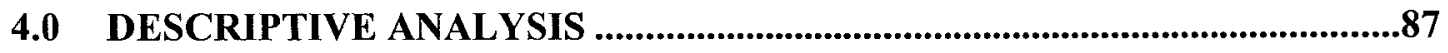

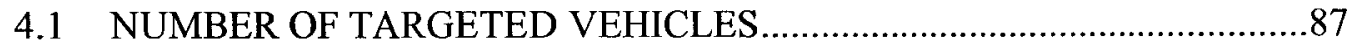

4.2 VEHICLE MOVEMENT DISTRIBUTIONS ON RL AND SCL .................89

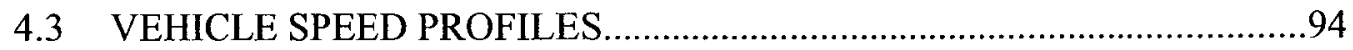

4.4 GEOMETRIC FEATURES ............................................................

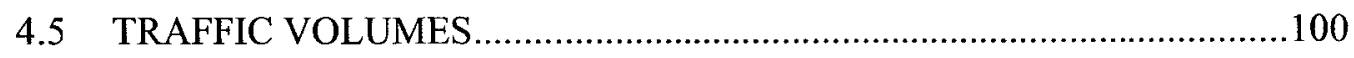

4.5.1 Equivalent Hourly Traffic ......................................................100

4.5.2 Average Annual Daily Traffic (AADT) ...................................105

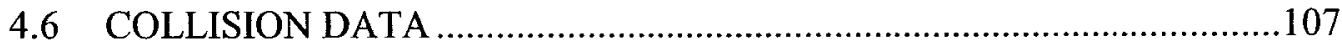

4.7 AGGREGATED DRIVER BEHAVIOUR MEASURES ........................108

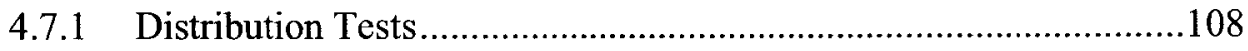

4.7.2 Test for Detecting Outliers....................................................109

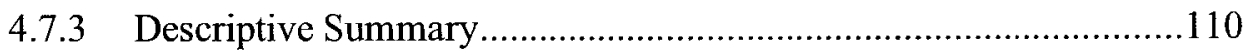

vii 
4.7.4 Distribution of Targeted Heavy Vehicles .....................................112

4.7.5 Distribution of RL Mean Speeds ................................................116

4.7.6 Distribution of Speeds on SCL ...............................................118

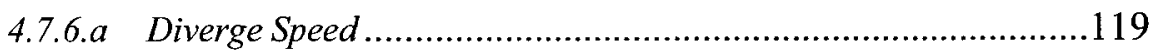

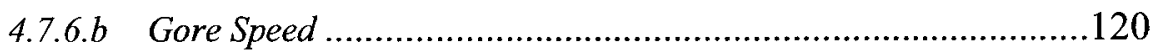

4.7.7 Distribution of Deceleration Rates on SCL ................................122

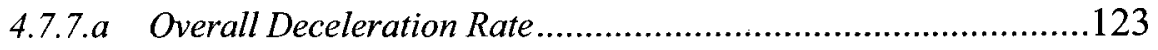

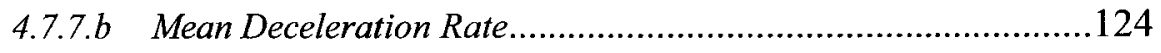

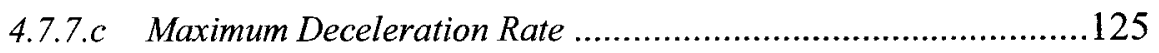

4.7.8 Distribution of Effective Deceleration Distance............................126

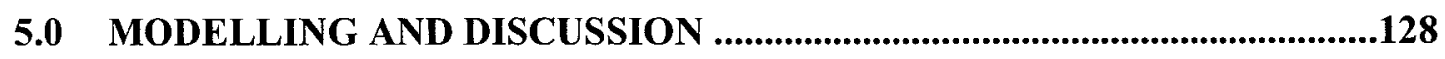

5.1 FREEWAY RIGHT-LANE MEAN SPEED MODELS ….......................131

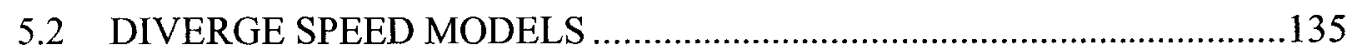

5.2.1 Diverge Speed Models for Combined SCLs................................135

5.2.2 Diverge Speed Models for Limited SCLs..................................138

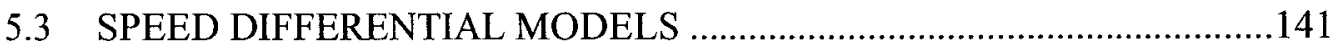

5.3.1 Speed Differential Models for Combined SCLs ...........................141

5.3.2 Speed Differential Models for Limited SCLs .............................143

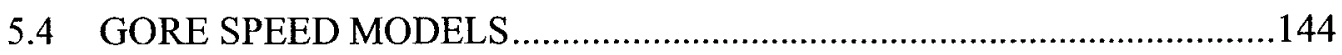

5.4.1 Gore Speed Models for Combined SCLs.....................................144

5.4.2 Gore Speed Models for Limited SCLs.......................................146

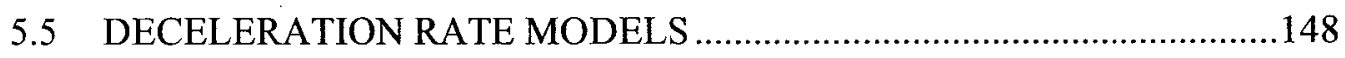

5.5.1 Overall Deceleration Models for Combined SCLs .......................149

viii 
5.5.2 Overall Deceleration Models for Limited SCLs..........................151

5.5.3 Maximum Deceleration Models for Combined SCLs ....................152

5.6 EFFECTIVE DECELERATION DISTANCE MODELS...........................154

5.6.1 Effective Deceleration Distance Models for Combined SCLs ........155

5.6.2 Effective Deceleration Distance Models for Limited SCLs ............156

6.0 APPLICATION OF DEVELOPED MODELS ........................................158

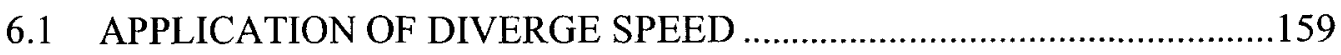

6.2 APPLICATION OF SPEED DIFFERENTIAL ........................................162

6.3 APPLICATION OF GORE SPEED ....................................................166

6.4 APPLICATION OF OVERALL DECELERATION RATE …...................168

6.5 APPLICATION OF MAXIMUM DECELERATION RATE ....................170

6.6 APPLICATION OF EFFECTIVE DECELERATION DISTANCE ............174

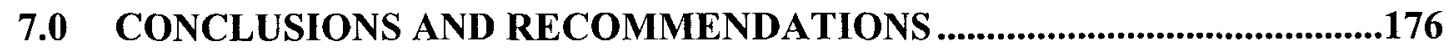

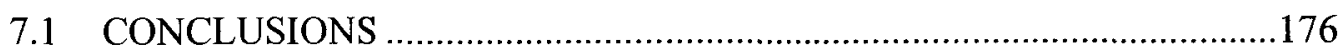

7.2 RECOMMENDATIONS FOR FUTURE RESEARCH ...........................181

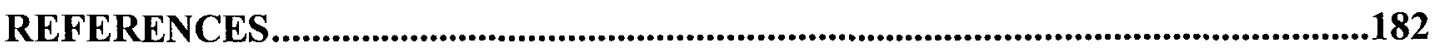

APPENDIX A: VEHICLE MOVEMENT DISTRIBUTION ON SELECTED

STUDY SITES .............................................................................................................191

APPENDIX B: VEHICLE SPEED PROFILES ON DECELERATION SPEED

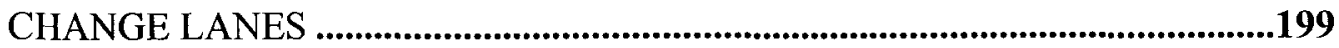

APPENDIX C: DESCRIPTIVE SUMMARY OF SPEEDS, DECELERATION

RATES, AND EFFECTIVE DECELERATION DISTANCES ON SELECTED

STUDY SITES 
APPENDIX D: SUMMARY OF ADDITIONAL SIGNIFICANT MODELS FOR COMBINED SPEED CHANGE LANES .........................................................219 APPENDIX E: SUMMARY OF ADDITIONAL SIGNIFICANT MODELS FOR LIMITED LENGTH TYPE SPEED CHANGE LANES.....................................222 


\section{LIST OF TABLES}

Table 2.1: Ramp Design Speed (TAC 1999, pp. 2.4.6.1) ..........................................18

Table 2.2: Design Length for Deceleration (TAC 1999, pp. 2.4.6.3) .............................24

Table 2.3: Minimum Deceleration Lane Lengths for Exit Terminals with Flat Grades

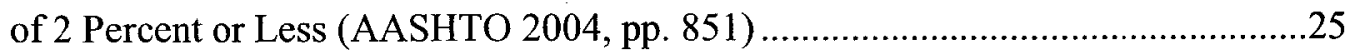

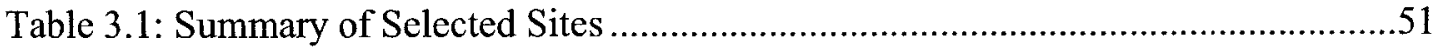

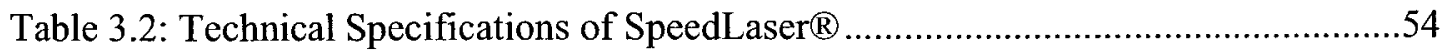

Table 3.3: Worksheet Sample for Recording Vehicles' Class and Movement..................56

Table 3.4: Average Heights of Front and Back Reflective Sides for PCs \& HVs............60

Table 4.1: Summary of Geometric Data for Exit Ramp Terminals Selected for this

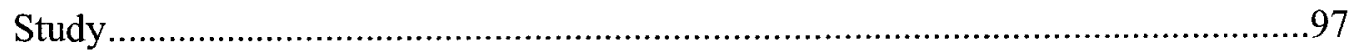

Table 4.2: Descriptive Summary of Geometric Data for Exit Terminals .........................98

Table 4.3: Distributive Summary Sample for Speeds on Bronson (W-NS) ....................111

Table 4.4: Distributive Summary Sample for Deceleration Rates and Effective

Deceleration Distances on Bronson (W-NS) .................................................111

Table 5.1: RL Mean Speed Predictive Models Summary for Limited SCLs ..................133

Table 5.2: Diverge Speed Predictive Models Summary for Combined SCLs..................136

Table 5.3: Diverge Speed Predictive Models Summary for Limited SCLs...................139

Table 5.4: Speed Differential Predictive Model Summary for Combined SCLs ............141

Table 5.5: Speed Differential Predictive Model Summary for Limited SCLs ................143

Table 5.6: Gore Speed Predictive Model Summary for Combined SCLs .....................145

Table 5.7: Gore Speed Predictive Models Summary for Limited SCLs........................146

Table 5.8: Overall Deceleration Predictive Models Summary for Combined SCLs.......149

$\mathrm{xi}$ 
Table 5.9: Overall Deceleration Predictive Model Summary for Limited SCLs

Table 5.10: Maximum Deceleration Predictive Model Summary for Combined SCLs..153

Table 5.11: Effective Dec. Distance Predictive Model Summary for Combined SCLs..155

Table 5.12: Effective Dec. Distance Predictive Model Summary for Length SCLs .......156 


\section{LIST OF FIGURES}

Figure 2.1: Basic Interchange Configurations (AASHTO 2004, pp. 744) ...........................9

Figure 2.2: Capacity of Diverge Areas (HCM 2000, pp. 13-23) ....................................12

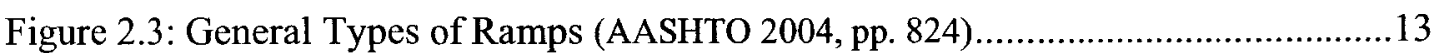

Figure 2.4: Ramp Terminal Spacing (TAC 1999, pp. 2.4.6.16).................................... 14

Figure 2.5: Consistency of Exits (TAC 1999, pp. 2.4.4.3) .............................................

Figure 2.6: Typical Gore Characteristics (AASHTO, 2004: pp. 10-59).............................19

Figure 2.7: Deceleration Speed-Change Lane Types (TAC 1999, pp. 2.4.6.4) ...................20

Figure 2.8: Alternative Methods of Dropping Auxiliary Lanes (AASHTO 2004, pp. 815) 22

Figure 2.9: Length of Freeway Weaving Section (HCM 2000, pp. 13-18) ........................27

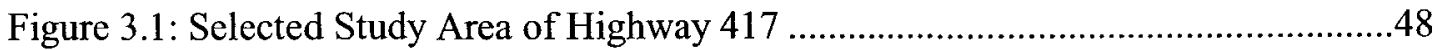

Figure 3.2: SpeedLaser ${ }^{\circledR}$ and Accessories, Laser Atlanta ..........................................53

Figure 3.3: Field Photo of an Extended Length Type SCL, St. Laurent Blvd (W-NS).....58

Figure 3.4: Field Photo of a Limited Length Type SCL, Carp Rd (E-NS) .......................58

Figure 3.5: SpeedLaser ${ }^{\circledR}$ Speed Data Readings File Sample .......................................62

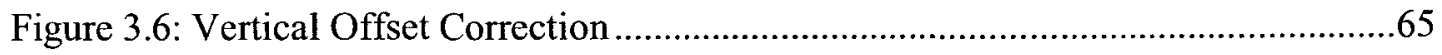

Figure 3.7: Vertical and Horizontal Offset Corrections...........................................65

Figure 3.8: Right-Lane Corrected Speed Profile Sample .......................................... 73

Figure 3.9: Speed-Change Lane Corrected Speed Profile Sample ..................................75

Figure 3.10: Analysis Parameters Summary for all Targeted Vehicles, Carp (E-NS) .........77

Figure 3.11: Analysis Parameters Summary for all Targeted Vehicles, Greenbank (E-NS) 77

Figure 3.12: Sample of ETR Map - With Marking, MTO ….....................................80

Figure 3.13: A Sample of ETR Map - Without Marking, MTO ....................................80

xiii 
Figure 4.1: Frequency Distribution of Number of Vehicles on SCL and RL .88

Figure 4.2: Sample Distribution of Vehicles and their Movement on Bronson (W-NS).....90 Figure 4.3: Sample Distribution of Vehicles and their Movement on St. Laurent (W-NS) 90 Figure 4.4: Sample Distribution of Vehicles and their Movements on Woodroffe (E-NS).93 Figure 4.5: Speed Profiles of All Vehicle Targeted on Vanier Pkwy (W-NS) .................95

Figure 4.6: Speed Profiles of All Vehicle Targeted on Bronson Ave (W-NS).................96

Figure 4.7: Distribution of Equivalent Hourly Traffic Volumes on RLs ......................101

Figure 4.8: Distribution of Equivalent Hourly Traffic Volumes on SCLs .....................101

Figure 4.9: Distribution of Vehicle Classification Percentages on RL-Upstream...........104

Figure 4.10: Distribution of Vehicle Classification Percentages on RL-Gore ................104

Figure 4.11: Distribution of Vehicle Classification Percentages on SCL (Ramp) ..........105

Figure 4.12: Comparison of Peak and Off-Peak Hour Traffic Volumes on Mainline.....106

Figure 4.13: Comparison of Peak and Off-Peak Hour Traffic Volumes on Ramp..........106

Figure 4.14: Frequency Distribution of Collisions ................................................ 108

Figure 4.15: Sample and Population $85^{\text {th }}$ Percentile for Diverge-Speed ........................112

Figure 4.16: Combined PC \& HV vs. PC Only for $85^{\text {th }}$ RL Mean Speed ......................113

Figure 4.17: Combined PC \& HV vs. PC Only for $85^{\text {th }}$ Diverge Speed..........................114

Figure 4.18: Combined PC \& HV vs. PC Only for $85^{\text {th }}$ Gore Speed.............................114

Figure 4.19: Combined PC \& HV vs. PC Only for $85^{\text {th }}$ Overall Deceleration Rate........115

Figure 4.20: Combined PC \& HV vs. PC Only for $85^{\text {th }}$ Effective Deceleration Distance 115

Figure 4.21: Distribution of RL Mean Speeds of All Vehicle Movements .....................116

Figure 4.22: Distribution of RL Mean Speeds of Through Vehicles Only ....................117

Figure 4.23: Comparison between All Movement and Through RL Mean Speeds........118

xiv 
Figure 4.24: Frequency Distribution of Speeds on SCL 119

Figure 4.25: Distribution of Diverge Speed on SCL ............................................. 120

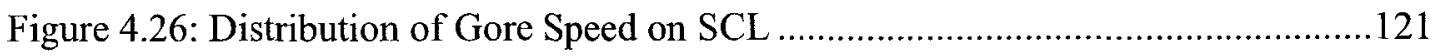

Figure 4.27: Frequency Distribution of Deceleration Rates on SCL ..........................122

Figure 4.28: Distribution of Overall Deceleration Rate on SCL ................................ 124

Figure 4.29: Overall and Mean $85^{\text {th }}$ Percentile Deceleration Rates...............................125

Figure 4.30: Distribution of Maximum Deceleration Rate on SCL.............................126

Figure 4.31: Distribution of Effective Deceleration Distance on SCL ........................127

Figure 4.32: Frequency Distribution of $85^{\text {th }}, 95^{\text {th }}$, Mean Effective Deceleration Distance, and SCL Lengths

Figure 6.1.a: Application of Diverge Speed Based on Length of Deceleration SCL and

Diverging Traffic Volume $\left(V_{R L}=80 \mathrm{~km} / \mathrm{h}\right)$. 159

Figure 6.1.b: Application of Diverge Speed Based on Length of Deceleration SCL and

Diverging Traffic Volume $\left(V_{R L}=90 \mathrm{~km} / \mathrm{h}\right)$.

Figure 6.1.c: Application of Diverge Speed Based on Length of Deceleration SCL and

Diverging Traffic Volume $\left(V_{R L}=100 \mathrm{~km} / \mathrm{h}\right)$

Figure 6.1.d: Application of Diverge Speed Based on Length of Deceleration SCL and

Diverging Traffic Volume $\left(V_{R L}=110 \mathrm{~km} / \mathrm{h}\right)$ 161

Figure 6.1.e: Application of Diverge Speed Based on Length of Deceleration SCL and

Diverging Traffic Volume $\left(V_{R L}=120 \mathrm{~km} / \mathrm{h}\right)$.

Figure 6.2.a: Application of Speed Differential Based on Length of Deceleration SCL and

RL Average Traffic Volume $\left(L_{t}=10 \mathrm{~m}\right)$ 163 
Figure 6.2.b: Application of Speed Differential Based on Length of Deceleration SCL and RL Average Traffic Volume $\left(L_{t}=45 \mathrm{~m}\right)$ 164

Figure 6.2.c: Application of Speed Differential Based on Length of Deceleration SCL and RL Average Traffic Volume $\left(L_{t}=80 \mathrm{~m}\right)$ 164

Figure 6.2.d: Application of Speed Differential Based on Length of Deceleration SCL and RL Average Traffic Volume $\left(L_{t}=115 \mathrm{~m}\right)$ 165

Figure 6.3: Application of Gore Speed Based on Diverging Traffic Volume and Divergence Angle 167

Figure 6.4: Application of Overall Deceleration Rate Based on RL Average Traffic

Volume and Divergence Angle

Figure 6.5.a: Application of Maximum Deceleration Rate Based on RL Average Traffic

Volume, Speed Differential, and SCL Type $(\mathrm{HV} \%=6)$.

Figure 6.5.b: Application of Maximum Deceleration Rate Based on RL Average Traffic

Volume, Speed Differential, and SCL Type $(H V \%=10)$ 171

Figure 6.5.c: Application of Maximum Deceleration Rate Based on RL Average Traffic

Volume, Speed Differential, and SCL Type $(\mathrm{HV} \%=14)$ 172

Figure 6.5.d: Application of Maximum Deceleration Rate Based on RL Average Traffic

Volume, Speed Differential, and SCL Type $(H V \%=20)$ 172

Figure 6.6: Application of Effective Deceleration Distance Based on SCL Length and

Heavy Vehicles Percentage (HV\%)

xvi 


\section{CHAPTER ONE \\ 1.0 INTRODUCTION}

\subsection{BACKGROUND}

The safe and efficient movement of people and goods from one place to another is the main purpose of the road network. According to the respective function in terms of the character of a roadway, the road network is classified into four basic groups; namely: locals, collectors, arterials, and freeways. The Highway Capacity Manual (HCM 2000) defines a freeway as a divided highway with full control of access and two or more lanes for the exclusive use of traffic in each direction. Generally, a freeway has no traffic lights, stop signs, or any other regulations requiring travelling vehicles to stop for crossing traffic at-grade intersections.

Access to and exit from a freeway are constrained to the use of interchange to guarantee uninterrupted flow conditions. An interchange is a road junction that utilizes grade separation to permit traffic on a crossing roadway to pass through the junction without interrupting the freeway main traffic stream. According to the Geometric Design Guide for Canadian Roads of the Transportation Association of Canada (TAC 1999), the basic function of an interchange is to facilitate the safe and efficient movement of vehicles to and from a freeway. Generally, interchanges may connect two freeways, a freeway with an arterial, or two arterial roadways. For any of these cases, interchanges use roadway links, known as loops and ramps, to provide access to and exit from freeways.

The loops and ramps that form a specific interchange are usually designed to help the safe and efficient traffic operation of vehicles to and from freeways. The entrance of 
drivers to and their exit from the freeway is accomplished through on-ramp (entrance terminal) and off-ramp (exit terminal), which permit travellers to proceed to their destinations in an organized and efficient driving manner. There are many factors that affect the freeway traffic operation and safety performance on these areas. Most importantly, the distances available for travellers to accelerate, decelerate, or change lanes while entering, exiting or performing lane change manoeuvres on a freeway are common factor affecting freeway operational and safety performance.

On a freeway, an acceleration speed-change lane (SCL) always follows an entrance ramp terminal while a deceleration SCL always precedes an exit ramp terminal. Typically, a freeway merge area would follow an entrance ramp while a freeway diverge area would go before an exit ramp. Freeway diverge areas with adequate lengths of SCLs adjoining the freeway basic lanes should enable exiting vehicles to diverge off the freeway flow traffic, decelerate to the advisory ramp speed, and exit the freeway in a safe and relaxed manner. Relatively, short distances between freeway interchanges would not enable highway designers to provide adequate SCL lengths that drivers could efficiently and safely use to enter or exit a freeway.

\subsection{PROBLEM DEFINITION}

The 1920s saw the origin of the concept of controlled access facilities for movement of vehicular traffic at high speeds with isolated grade separations. From that time up until today, there has been a continuing evolution in geometric design of freeways and interchanges. Nonetheless, as noted by Koepke (1993), many of the design criteria for interchanges are based on data that is 50 to 60 years old. During last decades 
and with an effort to improve and increase the efficiency of the freeway deceleration SCL and the associated exit ramp, relatively few researchers, outstandingly Fisher (1948), Miller Jr. (1951), and Conklin (1959), have made exhaustive studies of vehicle operating characteristics in an attempt to establish the safest and most efficient design criteria. With such limited research of SCL design, it becomes obvious that more studies are still required. Inconveniently, the interchange design criteria, derived from data collected back in the 1940's and 1950's, are still reflected in the most recent editions of geometric design guides of highways and streets published by the American Association of State Highway and Transportation Officials (AASHTO 2004) and the Transportation Association of Canada (TAC 1999). Understandably then, the applicability of such data and research findings to current drivers, driving patterns, or vehicles may be legitimately questioned.

There are numerous factors that play a role in causing freeway collisions including driver behaviour, freeway design, weather and other factors. The most widely discussed factor related to freeway traffic collisions is the speed of drivers, Kockelman et al. (2004). Hutabarat (2004) also mentioned that the most significant source of driver error on roadways found to be excessive speed. Since choice of speed (noteworthy cause of driver error) has a direct impact on safety and is undeniably the element under full control by the road users, research studies have shown that traffic collisions not always occur in a direct proportional manner to drivers' error. This means that, in addition to road users' error, road characteristics play a major role in collision occurrence on the freeway segments. In addition, Lum and Reagan (1995) stated that approximately $30 \%$ of the reasons behind collisions are associated with road related factors, and thus the 
designers, to a high extent, have responsibility towards the occurrence of unsafe driving manoeuvres.

The most obvious benefit of a freeway is the ease of travel that it provides to its users. Unfortunately, the benefit of travel can be adversely affected by the cost associated with road traffic collisions, which impose a huge public health burden worldwide. Thousands of people are killed on the world's roads each day, with several millions more injured due to traffic collisions. For example, during 2002 in the US, 42,815 deaths and almost three million non-fatal injuries were reported due to traffic collisions. According to Sleet and Branche (2004), traffic collisions are one of the leading causes of injuryrelated death worldwide. In fact, the total monetary costs of collisions are substantial. For example, costs for the year 2000 were estimated to be well over $\$ 200$ billion. In Canada, although there were considerable improvements in the roadway safety in the past decades, the Canadian Motor Vehicle Traffic Collision Statistics (Transport Canada 2004) reported that there were 2,730 people killed on the highway network with 212,347 injuries in only the year of 2004 .

Moreover, a number of research studies have identified interchanges as the most likely area for freeway collisions to occur, especially as it relates to the number and severity of collisions. For instance, based on data provided by the US Fatality Analysis Reporting System (FARS) and General Estimates System (GES), McCartt et al. (2004) reported that an estimated 82,609 interstate freeway collisions $(24,996$ injuries and 544 fatal) were reported at interchanges in year 2001. Although freeway interchanges approximately comprise less than $5 \%$ of the total mileage of freeways, the report further revealed that $18 \%$ of all interstate freeway crashes, $17 \%$ of injury crashes, and $11 \%$ of 
fatal crashes occurred at interchanges. Additionally, Janson et al. (1998) acknowledged that, in the US, as much as $30 \%$ of all freeway truck crashes occur on or near on or off ramps, not even taking into consideration an additional $10-15 \%$ that occur at the intersection of such ramps and surface streets.

\subsection{OBJECTIVES}

Driver behaviour evaluation at freeway diverge influence areas is the main objective of this research. Freeway-to-freeway connectors, which are usually facilitated through high speed ramps, are different from those connecting a freeway to an arterial roadway (i.e. exit ramp terminal). Exiting the freeway to arterials is usually associated with substantial change in speed. Speed disparities might still exist between the freeway through and exiting vehicles although ramps and SCLs are provided to allow speed transitions.

In examining the diverge areas of a freeway, issues related to speed and safety become more critical than those associated with freeway merge areas, due to the higher speeds involved with the deceleration lanes as opposed to acceleration lanes (where speed is gradually increasing). The length of the deceleration lane is critical to allow the safe reduction of speed within the designated area. As stated by Park et al. (2001), collisions are much more likely if the speed of the lead vehicle at a diverge area is underestimated. As mentioned earlier, most design guide criteria are decades old and therefore, efforts were performed in this study to examine various aspects of freeway deceleration SCLs in connection with diverge areas from both operational and safety performance points of 
view. Based on the aforementioned information, the key objectives of this research study were set as follows:

- Review relevant research related to freeway diverge areas and more specifically deceleration lane.

- Develop statistical models to assist in predicting driver speeds on freeway deceleration SCLs and the adjacent right lanes.

- Develop statistical models to assist in predicting deceleration behaviour and effective deceleration distances on freeway deceleration SCLs.

- Develop statistical models to assist in predicting collisions on the freeway diverge areas using five-year collision data.

\subsection{SCOPE OF RESEARCH}

The scope of this study is limited to freeway interchanges as interchanges are not only restricted for use with freeways. The study area selected contained 13 interchanges along Highway 417 located within the City of Ottawa, Ontario, Canada. The selected sites varied in terms of geometric features, traffic, and land-use. This would help capture the effect of length of deceleration SCLs and the prevailing traffic on driver behaviour due to that variation. The speed data collected for 3,098 vehicles on selected sites in this study are limited to dry pavement conditions and daytime. This means that wet pavement surface conditions and poor climate conditions such as rain, snow, or windy circumstances are not considered in this study. Moreover, the speed data collection was confined to free-flow traffic conditions during off-peak hour periods only. 


\subsection{THESIS ORGANIZATION}

After presenting the introduction of this study in Chapter 1.0, Chapter 2.0 presents a review of the design guide criteria with the literature review of the previous studies related to the thesis topic. Chapter 3.0 presents in detail the data collection and preparation process. Then, Chapter 4.0 shows the descriptive analysis of the database collected for this study. In Chapter 5.0, the regression modeling techniques for predicting various driving parameters on freeway deceleration SCLs and the adjacent right lanes are presented. Chapter 6.0 provides the application examples of the developed models. Finally, Chapter 7.0 presents a summary of the study findings with recommendations for future research. 


\section{CHAPTER TWO \\ 2.0 LITERATURE REVIEW}

This chapter covers a review of the current highway design guides and the previous relevant research that addressed the effect of different design criteria on driving behaviour on freeway diverge areas. Section 2.1 presents the review of current highway design guides of the Transportation Association of Canada (TAC 1999) and the American Association of State Highway and Transportation Officials (AASHTO 2004), as well as the Highway Capacity Manual (HCM 2000). Section 2.2 presents a review of the available literature related to freeway deceleration speed-change lanes (SCL) and freeway diverge areas. In addition, the literature review discuses the freeway safety performance from both an implicit and explicit points of view.

\subsection{REVIEW OF DESIGN GUIDES}

This section will provide a brief review of the design criteria and basic definitions provided by current design guides including AASHTO (2004) and TAC (1999). These primary design guides in North America were reviewed concerning interchange design including the freeway diverge areas, interchange ramp terminals, and SCLs.

\subsubsection{Design Criteria and Interchange Configurations}

An interchange is defined by TAC (1999) as "a system of interconnecting roadways in conjunction with one or more grade separations that provides for the movement of traffic between two or more roads on different levels". The obvious distinction between an interchange and a typical at-grade intersection is that an 
interchange allows for much higher traffic volumes on freeways with relatively safer operations and coordinated traffic flow at higher speeds. In addition, the traffic is protected from continual stops and starts that might be seen frequently in other road types. Freeway interchanges prevent crossing of different traffic streams as well as minimize turning conflicts, allowing a smoother flow between traffic movements in different travel directions. As mentioned in AASHTO (2004), interchange configurations include a wide variation ranging from simply one ramp connecting a surface street to the freeway to relatively complex designs that connect two or more freeways. Figure 2.1 illustrates different configurations of interchanges as presented in AASHTO (2004).

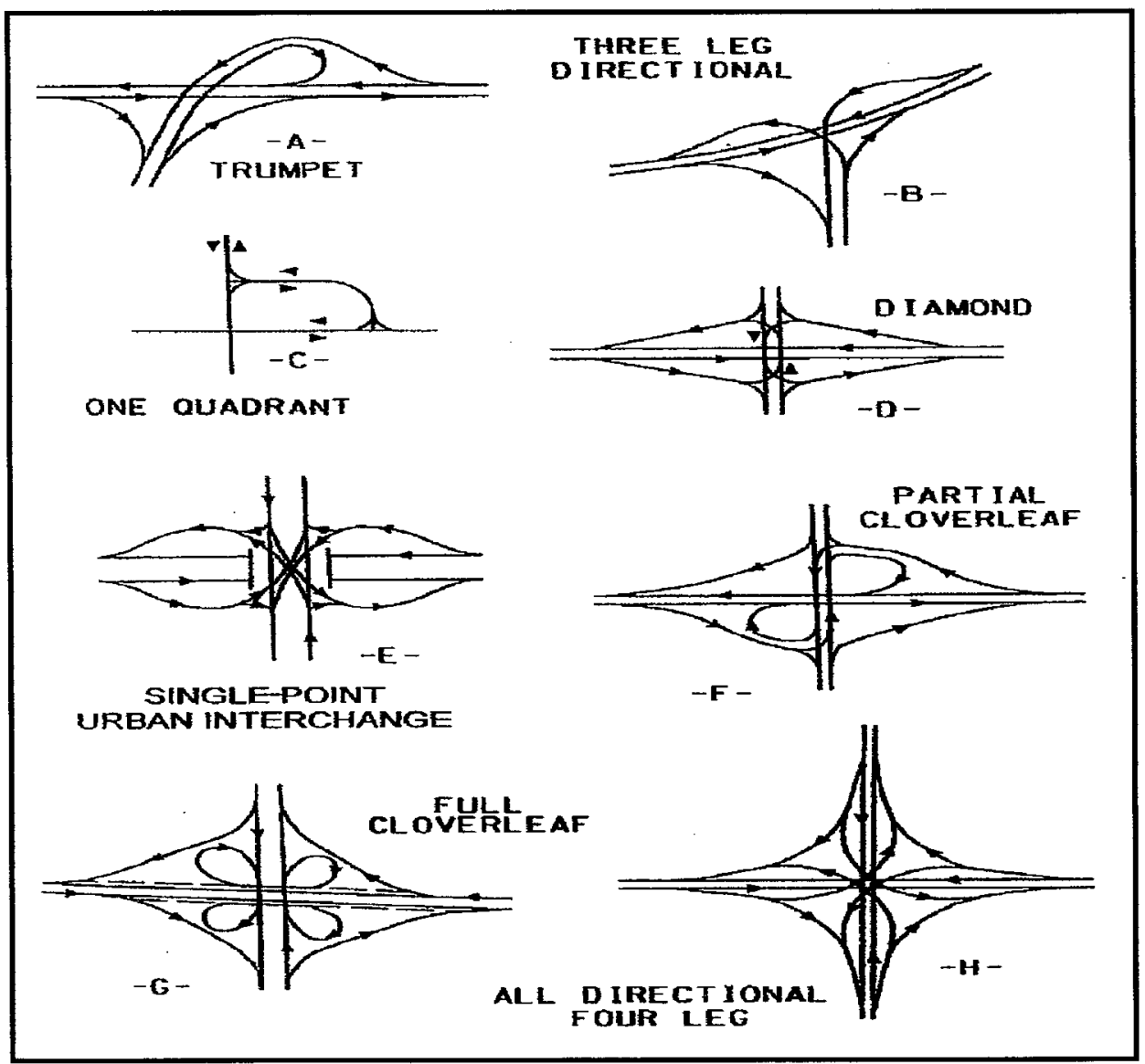

Figure 2.1: Basic Interchange Configurations (AASHTO 2004, pp. 744) 
Freeway interchanges are located at points along the roadway and these locations are typically based on two basic requirements. First requirement involves the need for additional interchanges order to provide additional access for communities in close proximity to the freeway. This is normally needed in central business district (CBD) or highly congested areas, where additional traffic flow would naturally necessitate interchanges. The second requirement is providing enough spacing for drivers to navigate properly that would not result if interchanges were located too closely together. Properly balancing these two diverse, but equally important, needs provides the appropriate design for an efficient freeway operation. Ideal spacing, as cited in TAC (1999), ranges from 3 to $8 \mathrm{~km}$ in rural areas, while in highly populated urban areas, such a lengthy separation between interchanges would not properly serve the surrounding land use. Subsequently, TAC (1999) recommends an optimal spacing of interchanges in urban areas of 2-3 km. There becomes a fine line between the need to provide freeway access to commuters in urban areas and safety concerns. "Freeway collision rates tend to increase as interchange spacing decreases in urban areas. This effect on collision rates is an important consideration in urban area interchange spacing". (TAC 1999, pp. 2.4.3.1)

Furthermore, AASHTO (2004) recommends even shorter distances between interchange spacing than the values mentioned in TAC (1999). These distances are stated to be at least $3 \mathrm{~km}$ in rural areas while a separation of only $1.5 \mathrm{~km}$ is suggested for the densely populated urban areas. However, collector roads could be utilized if there is a necessity for shorter distances between interchanges than the suggested values. Moreover, "the ideal average interchange spacing over a reasonable long section (8 to $10 \mathrm{~km}$ ) of freeway is $3 \mathrm{~km}$ or greater. The minimum average interchange spacing 
considered possible over a substantial length of freeway is $1 \mathrm{~km}$ ", (HCM 2000, pp. 10-6). Upon a quick review, it becomes obvious that there is no universally accepted 'best' distance between interchanges in either rural or urban settings.

\subsubsection{Freeway Diverge Areas}

Generally, a freeway diverge area precedes an off-ramp. The Highway Capacity Manual (HCM 2000) defines a freeway diverge area as "the area at which a single traffic stream is divided into two separate streams". At freeway diverge areas, vehicles separating from the freeway traffic flow initially occupy the deceleration lane and then reduce their speed from a higher speed on the freeway to a lower speed on the exit ramp. Practically, it is therefore critical for the highway designers to provide diverge areas with proper lengths to ensure the safe operations of vehicles exiting the freeway.

HCM (2000) provides an important method for studying diverge areas. In their assessment of evaluating freeway diverge areas, the primary methodology tends to limit the negative effects caused by the volume of exiting traffic. The freeway two through lanes adjacent to the SCL as well as the SCL itself are logically the most affected by the diverging traffic. Freeway through traffic vehicles in these areas might hence prefer to move away from those lanes, as long as volume permits them to do so. Based on HCM (2000), the effective range of these manoeuvres would extend $450 \mathrm{~m}$ from the physical gore of the exit ramp terminal.

Moreover, TAC (1999) indicates that there is no evidence so far that diverge manoeuvres affect the capacity of the freeway mainline. According to HCM (2000) view, the freeway capacity at a diverge area is either the capacity upstream the diverge area $\left(\mathrm{C}_{1}\right.$ 
in Figure 2.2) or the capacity of the freeway basic segment downstream the diverge area $\left(\mathrm{C}_{2}\right.$ in Figure 2.2$)$ in addition to the capacity of the exit ramp $\left(\mathrm{C}_{3}\right.$ in Figure 2.2). However, if the demand of exit volumes through the off-ramp exceeds the capacity, a failure will likely to occur. At the same time, HCM (2000) states that the flow occupying the first two lanes adjacent to the SCL including the off-ramp $\left(\mathrm{C}_{4}\right.$ in Figure 2.2$)$ cannot exceed $4400 \mathrm{pc} / \mathrm{h}$ for off-ramps.

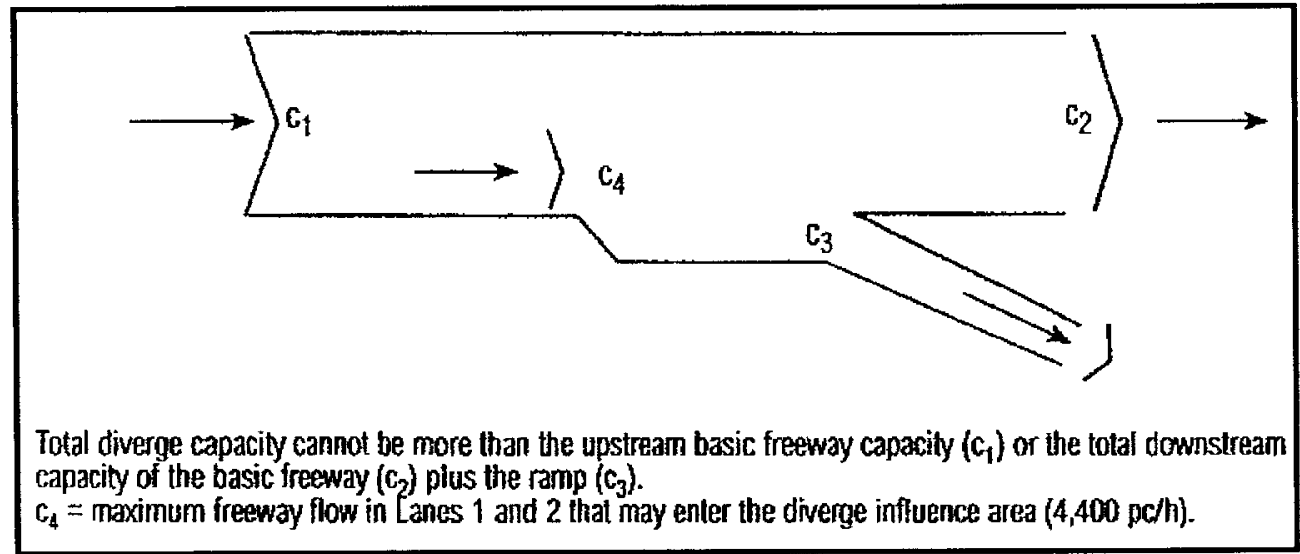

Figure 2.2: Capacity of Diverge Areas (HCM 2000, pp. 13-23)

\subsubsection{Interchange Ramp Terminals}

\subsection{3.a Definition}

TAC (1999) defines an interchange ramp as "a connecting roadway, usually oneway, carrying traffic between two grade-separated through roads". Interchange ramps have different configurations as shown in Figure 2.3. An interchange ramp consists of an exit terminal, the ramp itself (or a connecting roadway), and the entrance terminal. A freeway segment is described as the distance between two successive ramps. The spacing of these ramp terminals includes the distance between interchanges or within the same 
interchange (i.e. the entrance and exit ramps). Freeway segments are generally classified as four primary types: (1) entrance-entrance; (2) entrance-exit; (3) exit-entrance; and (4) exit-exit. These classifications are simply based on the type of the first and second ramp for each individual segment.

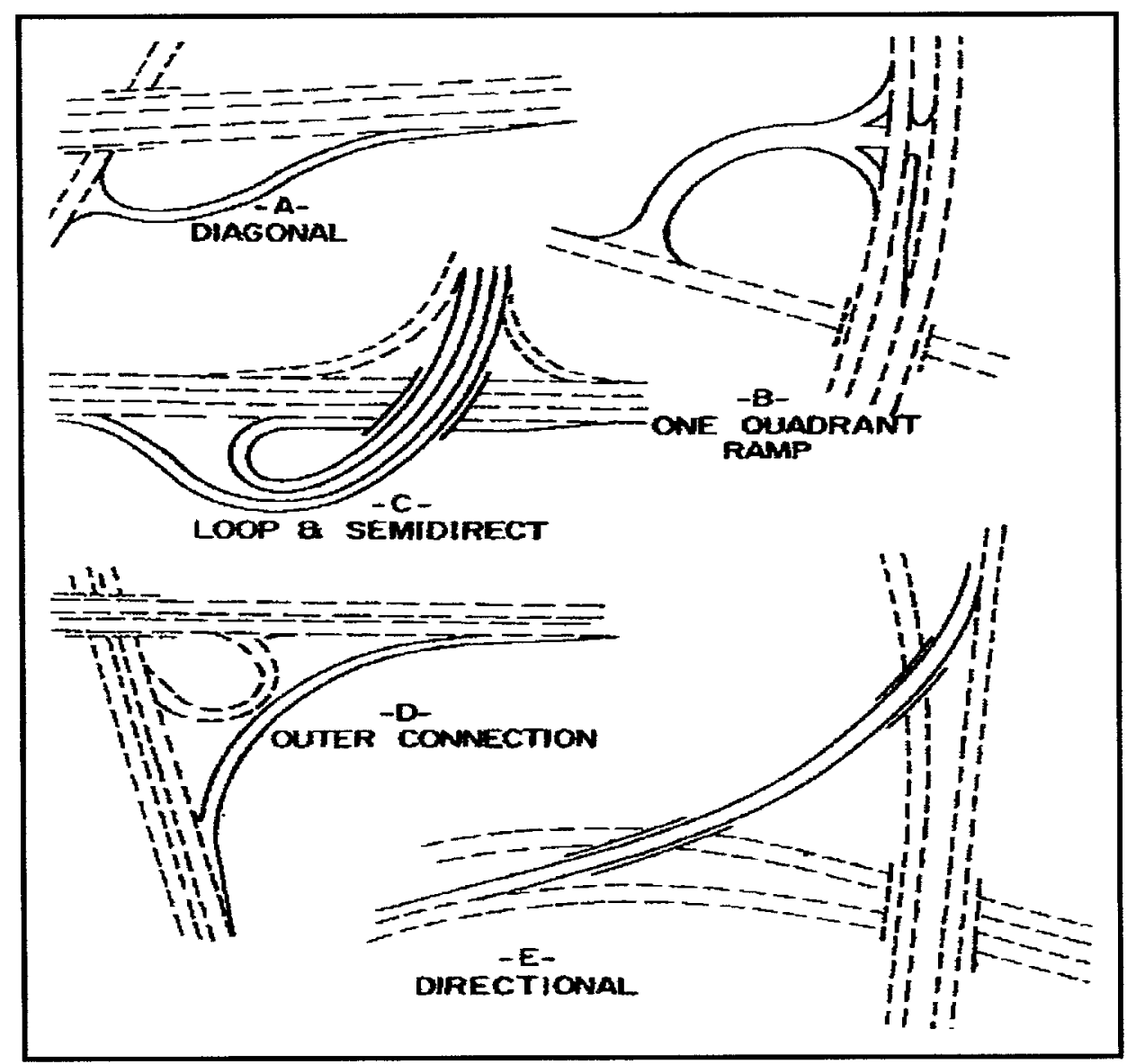

Figure 2.3: General Types of Ramps (AASHTO 2004, pp. 824)

The length of each freeway segment is measured from the physical nose at the gore of the first ramp to the physical nose at the gore of the second ramp - (see Section 2.1.3.d for definition of gore area and physical nose). The empirical values for ramp 
terminal spacing (i.e. distance between two successive ramp terminals) set by TAC (1999) are shown in Figure 2.4.

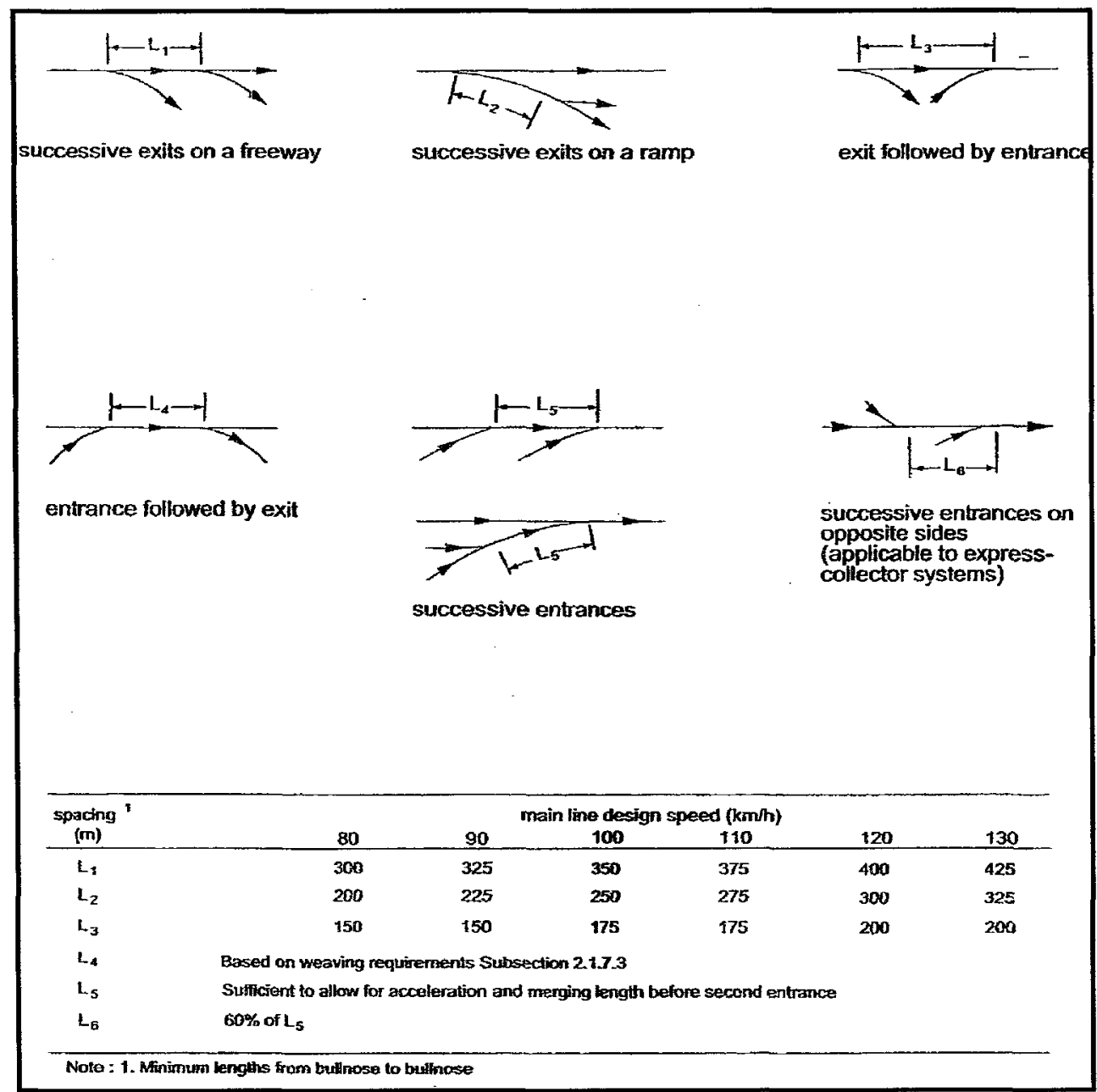

Figure 2.4: Ramp Terminal Spacing (TAC 1999, pp. 2.4.6.16)

The empirical values, published by TAC (1999) and shown in Figure 2.4, were based on various criteria to allow the freeway users to make safe and sufficient driving manoeuvres. These criteria are as follow:

- In the case of two successive exit terminals, the spacing distance should be based on the provision of adequate signing. 
- In the case of two successive entrances, the distance should be based on the merging manoeuvre length required for vehicles joining the freeway from the first entrance.

- In the case of an entrance followed by an exit terminal, the spacing should be based on the weaving requirements.

- In the case of an exit terminal followed by an entrance terminal, the length between these two terminals should be sufficient for vehicles on a through lane to be prepared for the next freeway merge area.

\subsection{3.b Design Consistency of Ramps}

The design guides (TAC and AASHTO) have always stressed on the importance of ramp consistency or uniformity, which is a special application of general design consistency concepts. Consistency between the ramps is one critical factor that should guarantee safe and smooth traffic flow on freeways. There are a number of ways to ensure ramp consistency along a freeway, including maintaining single exits ahead of structures on a specific freeway length. Due to the fact that they are more visible to the driver, which enables the selection of proper speeds and vehicle positioning, ramps located before overpass structures are preferred over exit ramps positioned after the structure. While left-hand exit ramps are used in some areas, TAC (1999) does not recommend such a design. From the standpoint of consistency, the right-hand ramp is much more widely known and anticipated by drivers. In an effort to maintain consistency, left-hand ramps should be avoided unless they are absolutely necessary to maintain the local traffic flow. 
Included in the recommendations of TAC (1999) is the importance of a consistent design for a series of interchanges on a specific section of a highway in order to increase driver comfort, which in turn would increase safety. TAC (1999) presented graphically two sets of consistent and inconsistent exit designs as seen in Figure 2.5.

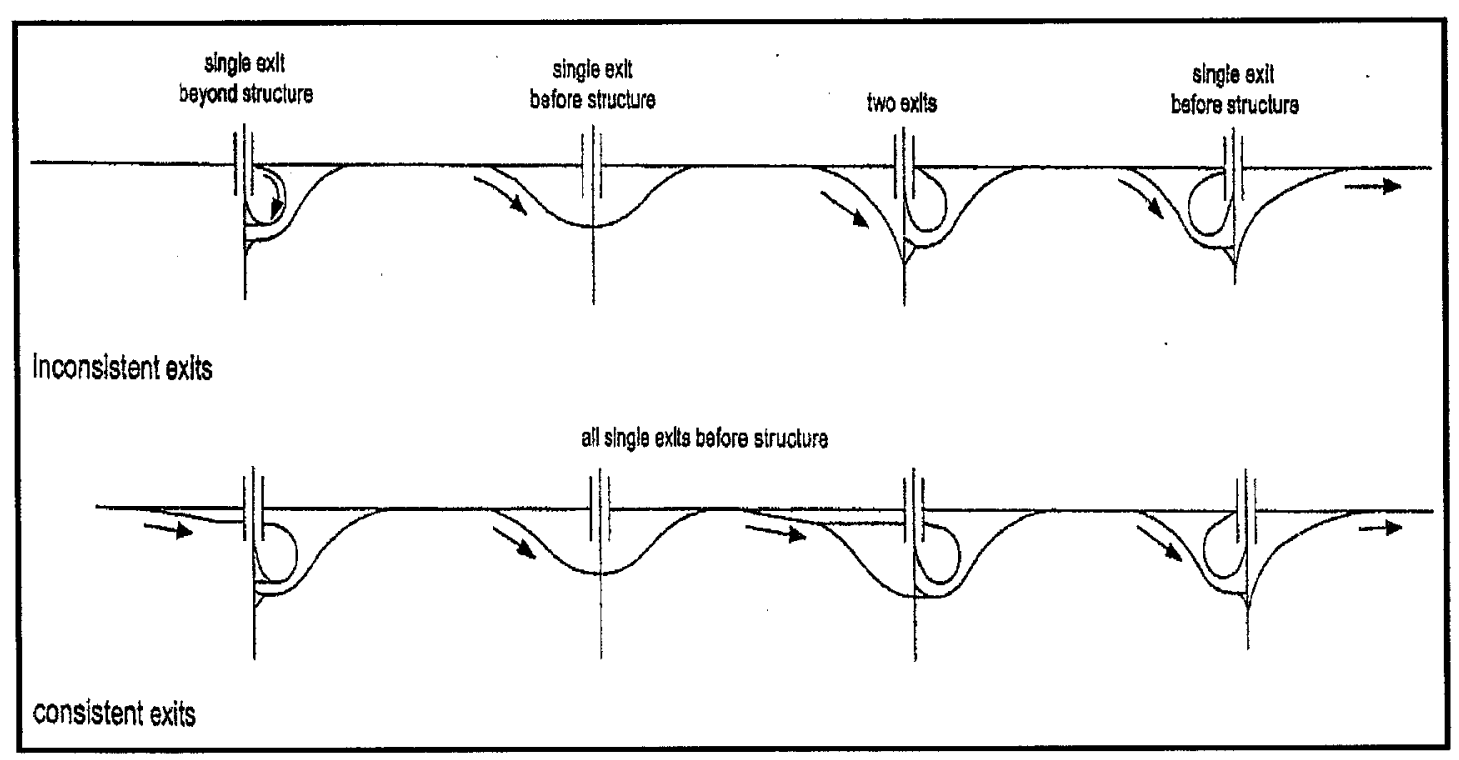

Figure 2.5: Consistency of Exits (TAC 1999, pp. 2.4.4.3)

In Figure 2.5, the consistency concept as described in TAC (1999) is illustrated. The design scheme at the top of this figure illustrates interchanges with a mix of single and double exits. The ramps are placed in some cases before the overpass structure, while in other cases they are located after the structure, which would lead to driver confusion. On the other hand, a consistent and uniform design scheme is further shown in the lower section of Figure 2.5. Each interchange in this second part has a single exit before the structure. This kind of consistency would enable the driver to maintain proper concentration without causing any confusion to the driver and hence, improved operation 
and safety on freeway. Therefore, it is desirable for interchanges on a freeway to have some degree of uniformity in exit design to support driver expectancy. This certainly emphasizes the crucial relationship between design consistency of different roadway elements along a freeway and safety. While a definitive relationship between interchange design features and safety has yet to be quantified (TAC 1999), the interest in explaining such a relationship has become more predominant in transportation engineering.

An identical concept is highlighted by AASHTO (2004) stating that designing a series of interchanges should consider the consistency of the interchanges in addition to each individual interchange. In fact, AASHTO (2004) uses the term "interchange uniformity" specifically to apply to the concept of using consistent exits and entrances on freeways. "Considering the need for high capacity, appropriate level of service, and maximum safety in conjunction with freeway operations, it is desirable to provide uniformity in exit patterns", (AASHTO 2004, pp. 807).

\subsection{3.c Ramp Width and Speed}

Design speeds of ramps must be carefully set in order to maintain the uniformity or consistency concept for freeways. Table 2.1 lists the recommended design speeds for ramps as covered in TAC (1999). Theoretically, the design speeds of ramps should be as close to the speed of the freeway as possible, making the transition of exiting from and entering the freeway as smooth as possible. For that reason, the design guide recommends the upper limits of the ramp design speeds in Table 2.1 (i.e. the design domain). TAC (1999) also recommends that the minimum ramp design speed should be $40 \mathrm{~km} / \mathrm{h}$. Suitable ramp widths, according to TAC (1999), range from $4.8 \mathrm{~m}$ to $5.0 \mathrm{~m}$ for 
single ramp designs. Ramps utilizing two lanes would more appropriately use a minimum width of 3.7 meters, and should be widened for controlling ramp curve of radius $>50 \mathrm{~m}$, TAC (1999).

Table 2.1: Ramp Design Speed (TAC 1999, pp. 2.4.6.1)

\begin{tabular}{|c|c|}
\hline $\begin{array}{c}\text { Roadway Design Speed } \\
(\mathbf{k m} / \mathbf{h})\end{array}$ & $\begin{array}{c}\text { Ramp Design Speed, Design Domain } \\
(\mathbf{k m} / \mathbf{h})\end{array}$ \\
\hline 60 & $50-40$ \\
\hline 70 & $60-40$ \\
\hline 80 & $70-40$ \\
\hline 90 & $80-50$ \\
\hline 100 & $90-50$ \\
\hline 110 & $100-60$ \\
\hline 120 & $110-60$ \\
\hline 130 & $110-70$ \\
\hline
\end{tabular}

\subsection{3.d Gore Area and Physical Nose}

AASHTO (2004) defines the term gore as "an area downstream from the shoulder intersection points" as shown in Figure 2.6. The physical nose is defined by AASHTO (2004) as "a point upstream from the gore, having some dimensional width, occurring at the separation of the roadways", while the painted nose is defined as " $a$ point, having no dimensional width, occurring at the separation of the roadways", (see Figure 2.6). AASHTO (2004) states that the geometric layout of such terms (gore, physical nose, and painted nose) is a significant part of the exit ramp terminal design. The gore area is considered as a decision point area where drivers should evidently see and understand the entire area as they approach the exit ramp terminal. It is desirable to have consistent gore areas that have same appearance to drivers along a freeway. The width at the gore nose is typically between $6.0 \mathrm{~m}$ to $9.0 \mathrm{~m}$ including the shoulders of both the 
mainline and ramp, but AASHTO (2004) recommends increasing these values if the speeds in excess of $100 \mathrm{~km} / \mathrm{h}$ are expected to be common.

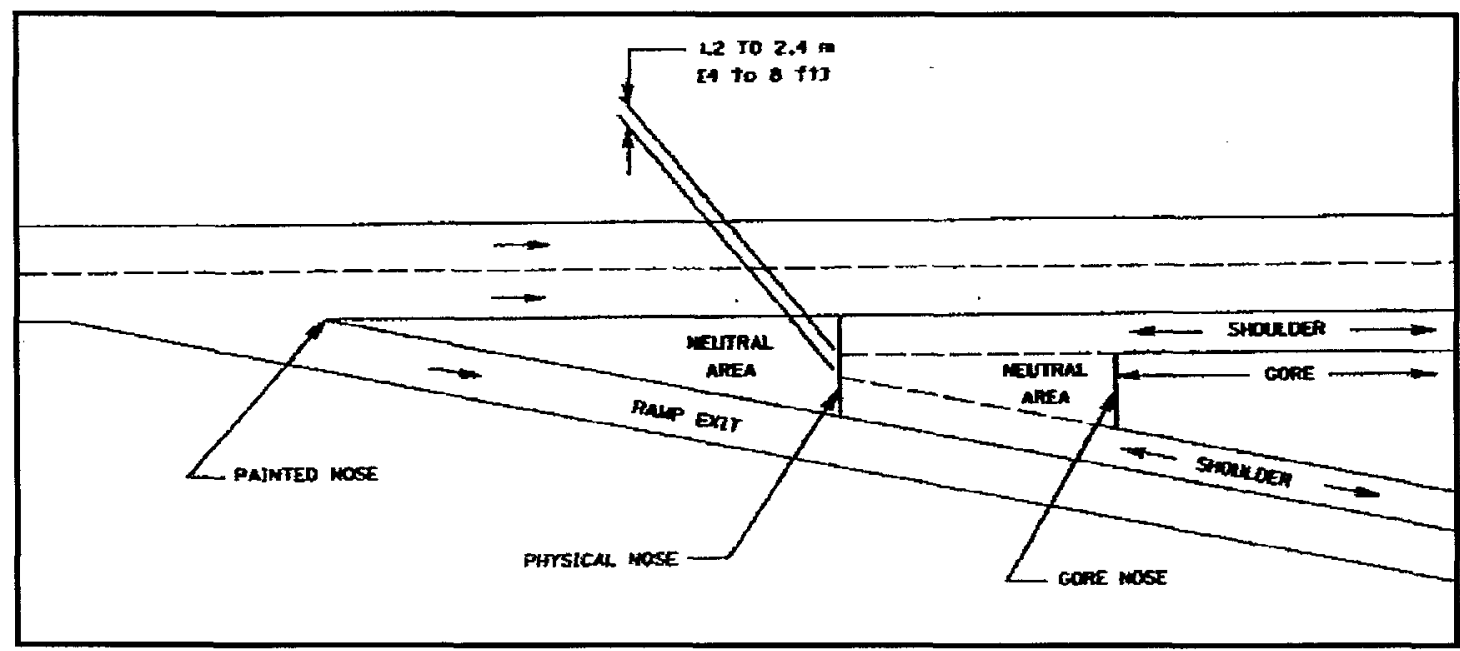

Figure 2.6: Typical Gore Characteristics (AASHTO, 2004: pp. 10-59)

The term "gore" generally refers to the area between a through roadway and a diverging exit ramp or a converging entrance ramp, as published by the TAC design guide. TAC (1999) referred to the gore as the "bullnose" and states that the collision rates near gore areas are higher than at other locations. For this reason, the gore area is recommended to be kept free of obstructions as possible and to offset the exit ramp away from the freeway main stream to provide a recovery area for errant vehicles. TAC (1999) did not suggest offset values at the bullnose, rather it states that such separations vary depending on the speeds of the roadway. 


\subsubsection{Speed-Change Lanes}

\subsection{4.a Definition}

TAC (1999) defines a speed-change lane (SCL) as "the added lane adjoining the travelled way of the roadway and does not necessarily imply a definite lane of uniform width". Figure 2.7 shows two types of deceleration SCLs as shown in the TAC (1999) design guide.

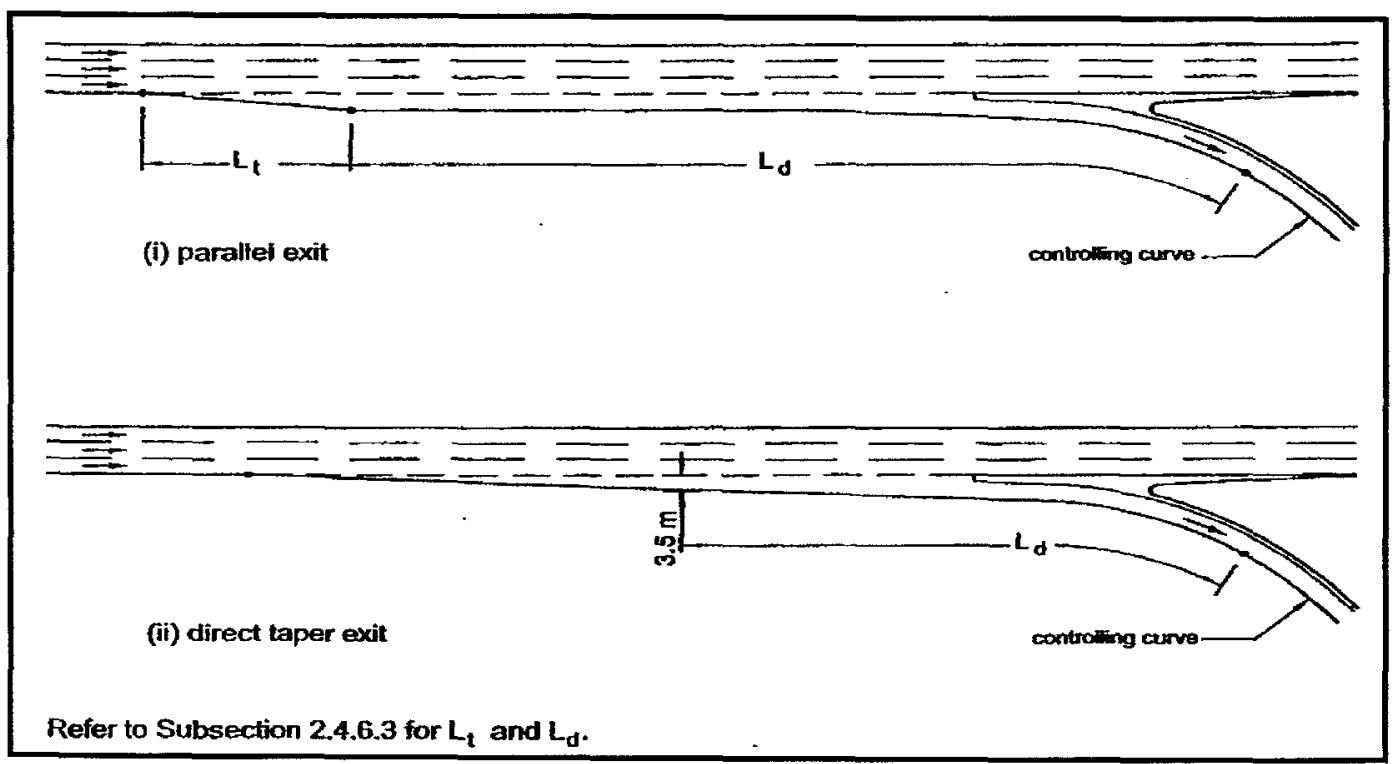

Figure 2.7: Deceleration Speed-Change Lane Types (TAC 1999, pp. 2.4.6.4)

The design guide also describes the SCL to be part of the elongated ramp terminal area. The length of a SCL needs to be sufficient to ensure the safe and comfort operation of any vehicle using it. TAC (1999) states that the length of a SCL is based on the following three factors:

1. The running speed on the through lanes - this is the speed at which drivers manoeuvre into a deceleration lane. 
2. The control speed of the ramp proper - this is the speed at which drivers drive the controlling ramp curve.

3. The manner of decelerating - this is the manner of which the drivers decelerate on the speed-change lane.

Characteristically, parallel and direct taper are the two types of speed-change lane designs used on freeways. The parallel SCL type is a limited lane that consists of the following two basic design elements; (1) $L_{d}$ : a constant width for gradual speed change between the freeway and the ramp, or (2) $L_{t}$ : a gradual change in width to guarantee an adequate rate of lateral movement. On the other hand, the direct taper SCL type is a limited lane that consists of the $L_{t}$ element to assure a gradual change in lane width along the lane's entire length. This second SCL type works on the principle of a direct entry or exit at a flat angel (TAC 1999).

The SCL was also described in AASHTO (2004) as an auxiliary lane including tapered area mainly for deceleration of vehicles leaving the through-traffic lanes of the freeway. AASHTO (2004) defined an auxiliary lane as "the portion of the roadway adjoining the travelled way for speed change". In general, the purpose of the SCL was stated "to comply with the concept of lane balance, to comply with capacity needs, or to accommodate speed changes, weaving, and manoeuvring of entering and leaving traffic" (AASHTO 2004, pp. 814).

It is important for a freeway to minimize the disturbance of the through traffic operation at diverge areas. This occurs using properly designed SCLs that enable drivers to make the necessary speed change between the freeway and the ramp in a comfortable manner. AASHTO (2004) also recommended that the width of the auxiliary lane should 
match the width of the through lane. Although some drivers use small portion of the available speed-change lanes, the lanes are still necessary to improve the flow of traffic on freeways, knowing that the extent of drivers' use of speed-change lanes depends on the freeway traffic volumes. AASHTO (2004) additionally comments that the use of SCLs with a long taper fits more the behaviour of most drivers.

In addition, AASHTO (2004) recommends the use of continuous auxiliary lanes between exit and entrance ramps in any of the following three cases: (1) when there are two interchanges that are close to each other, (2) when the distance between the end of the taper on the entrance terminal and the beginning of the taper on the exit terminal is relatively short, or (3) when local frontage roads do not exist. Figure 2.8 shows the basic alternative methods of dropping auxiliary lanes on freeways.

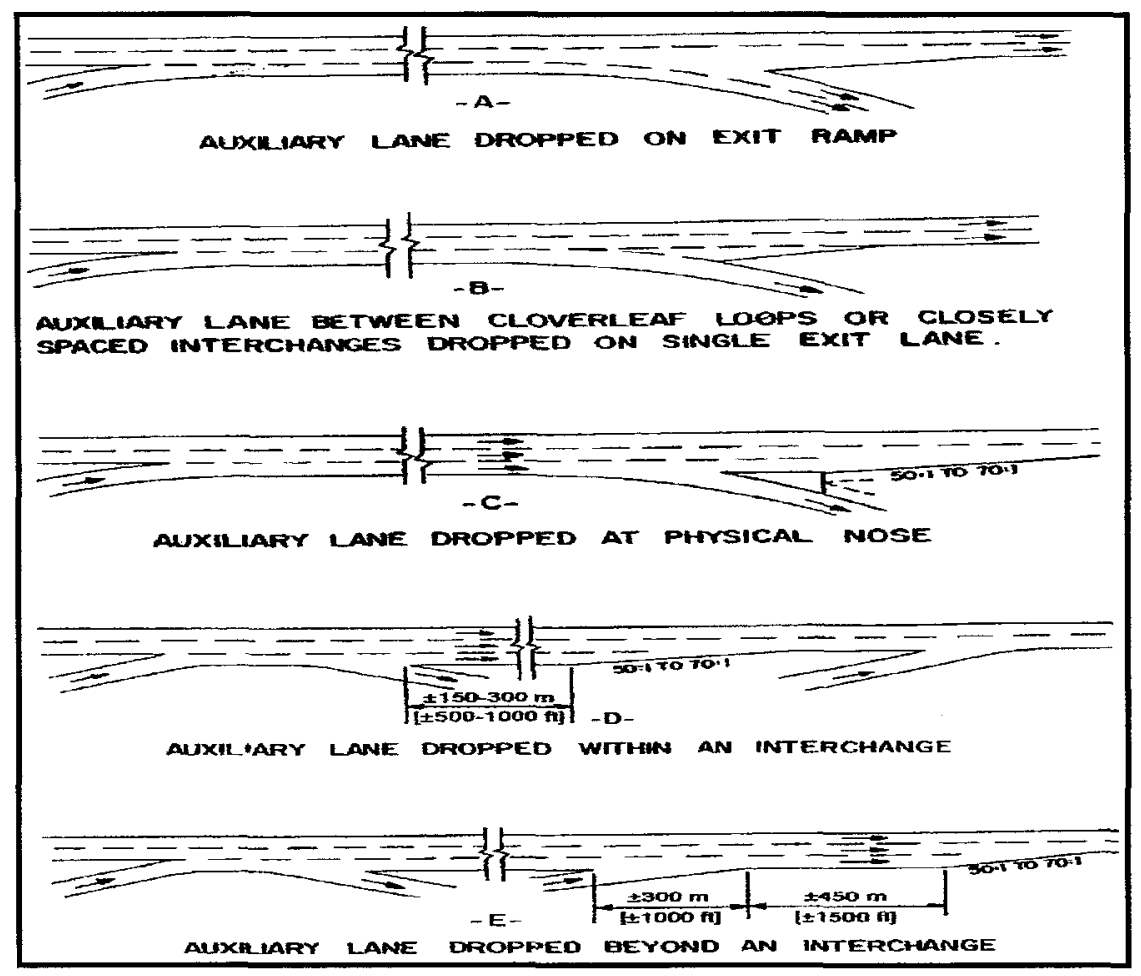

Figure 2.8: Alternative Methods of Dropping Auxiliary Lanes (AASHTO 2004, pp. 815) 
As shown in Figure 2.8, the auxiliary lane could be terminated by either a twolane exit ramp (Figure 2.8.A), or single-lane exit ramp (Figure 2.8.B). An additional method of terminating an auxiliary lane is to carry the full width auxiliary lane to the nose before tapering it into the throughway (Figure 2.8.C). In case a single-lane exit experience disturbance due to vehicles attempting to get back on the through lane, the recovery lane should be extended 150 to 300 meters before the taper (Figure 2.8.D). Whereas in the case of large interchanges, the design should extend the auxiliary lane for approximately 750 meters to allow for proper merging of vehicles onto the freeway (Figure 2.8.E).

Nevertheless, it was also stated in AASHTO (2004) that it is not exactly known what should be the effective length of the introduced auxiliary lane for any of the abovementioned circumstances. But experience indicates that a minimum distance of about $750 \mathrm{~m}$ for auxiliary lanes should produces the desired operational conditions, balance for the traffic load, and maintain a more uniform level of service on the freeway.

\subsection{4.b Length of Deceleration Lane}

According to TAC (1999), as mentioned earlier, the design domain for deceleration SCLs is based on three dominant factors for determining SCL lengths. Table 2.2 shows the design domain for lengths of deceleration lanes in single-lane exits on grades equal to or less than 3\% published by TAC (1999). Moreover, TAC (1999) stated that the third factor for determining the length of deceleration lane - the manner of deceleration - includes a domain of two different concepts. These two domains are 
provided on the basis of the lower and the upper values of the design domain shown in Table 2.2.

Table 2.2: Design Length for Deceleration (TAC 1999, pp. 2.4.6.3)

\begin{tabular}{|c|c|c|c|c|c|c|c|c|c|c|}
\hline \multicolumn{2}{|c|}{$\begin{array}{l}\text { Speed of Roadway } \\
(\mathrm{km} / \mathrm{h})\end{array}$} & \multirow{3}{*}{$\begin{array}{c}\text { Length of } \\
\text { Taper (m) } \\
L_{1}\end{array}$} & \multicolumn{8}{|c|}{$\begin{array}{l}\text { Length of Deceleration Lane Excluding Taper (m) } \\
\qquad L_{d}\end{array}$} \\
\hline \multirow[b]{2}{*}{ Design } & \multirow[b]{2}{*}{$\begin{array}{l}\text { Assumed } \\
\text { Operating }\end{array}$} & & \multicolumn{8}{|c|}{ Design Speed of Turning Roadway Curve $(\mathrm{km} / \mathrm{h})$} \\
\hline & & & $\begin{array}{c}\text { Stop } \\
\text { Condition }\end{array}$ & 20 & 30 & 40 & 50 & 60 & 70 & 80 \\
\hline $\begin{array}{l}60 \\
70 \\
80 \\
90 \\
100 \\
110 \\
120 \\
130\end{array}$ & $\begin{array}{c}55-60 \\
63-70 \\
70-80 \\
77-90 \\
85-100 \\
91-110 \\
98-120 \\
105-130\end{array}$ & $\begin{array}{c}55 \\
65 \\
70 \\
80 \\
85 \\
90 \\
95 \\
100\end{array}$ & $\begin{array}{c}90-115 \\
110-145 \\
130-170 \\
150-195 \\
170-215 \\
185-250 \\
200-320 \\
215-340\end{array}$ & $\begin{array}{c}85-110 \\
105-140 \\
120-165 \\
140-185 \\
160-210 \\
175-245 \\
190-315 \\
205-335\end{array}$ & $\begin{array}{c}80-105 \\
100-130 \\
115-160 \\
135-180 \\
155-205 \\
170-240 \\
185-310 \\
200-330\end{array}$ & $\begin{array}{c}70-90 \\
90-120 \\
105-150 \\
125-170 \\
145-200 \\
160-230 \\
180-305 \\
190-325\end{array}$ & $\begin{array}{c}55-60 \\
75-105 \\
95-130 \\
115-160 \\
135-185 \\
150-220 \\
170-300 \\
180-320\end{array}$ & $\begin{array}{c}60-70 \\
80-105 \\
100-135 \\
120-165 \\
140-205 \\
155-280 \\
170-300\end{array}$ & $\begin{array}{c}80-110 \\
100-145 \\
120-190 \\
135-270 \\
150-285\end{array}$ & $\begin{array}{l}100-165 \\
120-240 \\
135-270\end{array}$ \\
\hline Notes: & $\begin{array}{l}\text { 1. The se } \\
\text { 2. The uf } \\
\text { Highw }\end{array}$ & of of ral & based c & eratio & tance & $\begin{array}{l}2.4 .6 . \\
\text { es in th }\end{array}$ & $\begin{array}{l}\text { ald be } \\
\text { ometr }\end{array}$ & $\begin{array}{l}\text { red. } \\
\text { sign St: }\end{array}$ & ards for & ntario \\
\hline
\end{tabular}

For both design concepts, TAC (1999) assumes that the drivers diverge from the freeway through lanes onto the deceleration SCL and travel at the beginning of the SCL (start of taper section) at the operating speed. Afterward, the drivers are to maintain their operating speed until the end of taper in the case of parallel lane type or until the SCL is 3.5 meters in width in the case of taper lane type. On one hand, regarding the first design concept, TAC (1999) assumes that braking begins at the start of the parallel section (parallel type lane) or at the $3.5 \mathrm{~m}$ wide point (taper type lane) to decelerate to the ramp controlling speed. On the other hand, regarding the second design concept, TAC (1999) assumes that the vehicle travels for 2-4 seconds in gear without braking before performing a braking action in a relaxed manner until reaching the ramp controlling speed. 
For the case of two-lane exit terminals, the driver actions while exiting a freeway are different from those on a single-lane exit. TAC (1999) states that two-lane exit ramp terminals require more distance for SCLs than that for single-lane exit terminals. The determination of the SCL length with a two-lane exit ramp is based on a different concept and thereby the manoeuvre of the vehicle exiting using the left-hand lane of the two-lane exit ramp is governed by the ramp curve to the physical nose of the exit terminal. TAC (1999) mentions that for mainline roadway design speeds of $100 \mathrm{~km} / \mathrm{h}$ or greater, a SCL (including taper) of $400-450 \mathrm{~m}$ in length is recommended by both AASHTO and the Geometric Design Standards for Ontario Highways.

In addressing deceleration lanes and referring to AASHTO (2004), the minimum deceleration SCL lengths for various design speeds of both highway and ramp roadway as suggested by the design guide are shown in Table 2.3 .

Table 2.3: Minimum Deceleration Lane Lengths for Exit Terminals with Flat Grades of 2 Percent or Less (AASHTO 2004, pp. 851)

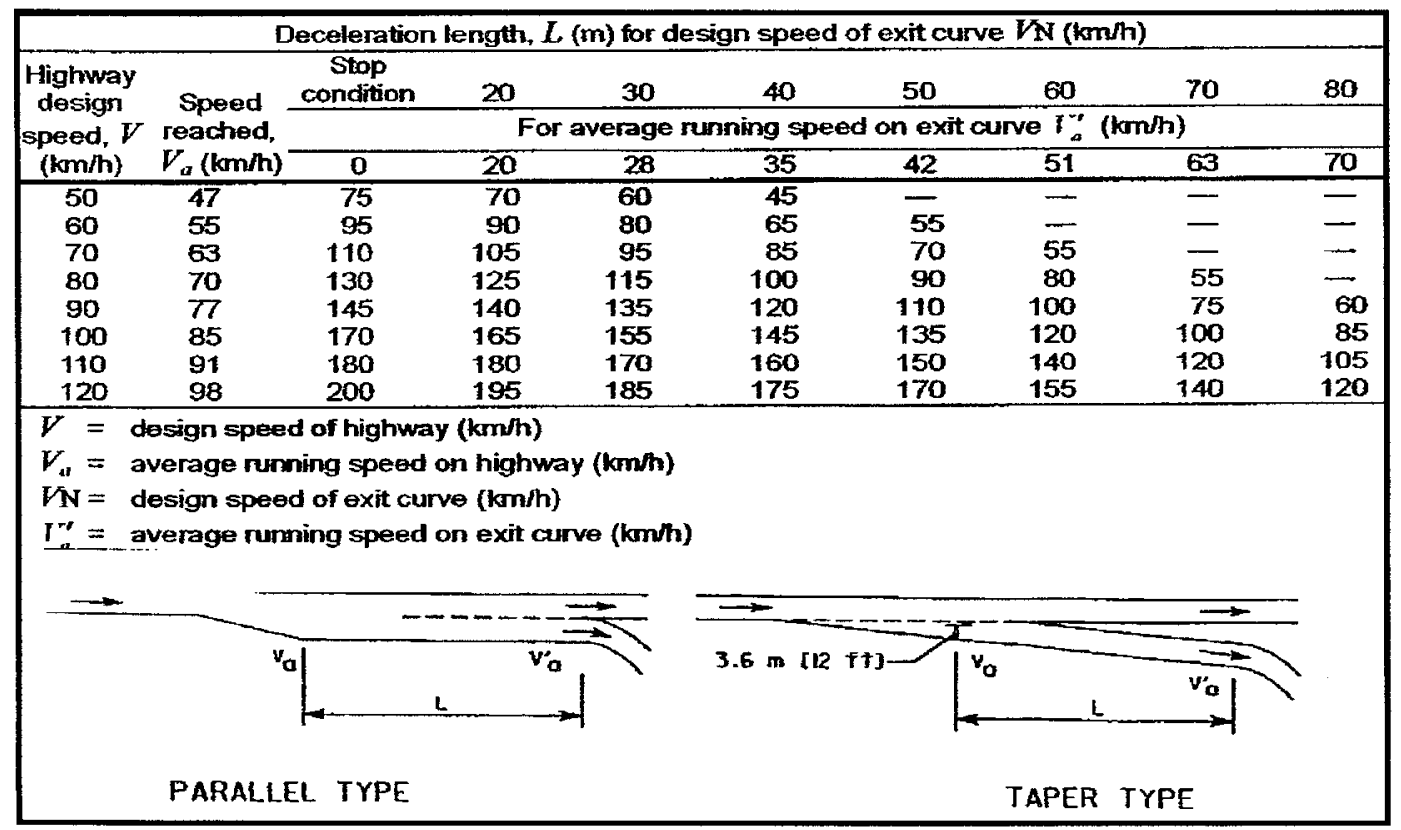


AASHTO (2004) recommends that the length of a parallel type deceleration lane should be at least 240 meters. However, the entire SCL is recommended to be $400-450 \mathrm{~m}$ for highway speeds of $100 \mathrm{~km}$ or above, with two lanes exiting the freeway. AASHTO (2004) further suggests that a short taper should be used on such ramps, since they allow drivers to be more aware of the approaching added lane. AASHTO (2004) states that the length of a taper type deceleration lane is usually measured from the point where the right edge of the tapered section is 3.6 meters away from the right edge of the freeway right most through lane to the point where the first horizontal curve on the ramp starts. On the other hand, the length of a parallel type deceleration lane is measured from the point where the added lane (that should attain a 3.6 meters width) to the point where the right edge of the freeway right most through lane intersects with the left edge of the exit ramp, AASHTO (2004).

TAC (1999) states that an auxiliary lane that is to be extended beyond an entrance terminal to the following exit ramp terminal should be continued for a distance of $700 \mathrm{~m}$ to $900 \mathrm{~m}$ to allow the entering and exiting traffic to merge into and diverge away from the freeway through lanes. The length of the weaving segment, as suggested by HCM (2000), is measured from a point where the right edge of the freeway through lane is 0.6 meters away from the left edge of the entrance ramp, to a point where the freeway right edge and the exit ramp left edge are 3.7 meters apart. An illustration of a weaving area is shown in Figure 2.9, as published by the HCM (2000). TAC (1999) similarly recommends a weaving measurement starting at the entrance gore, where the spacing between the two pavement edges (right edge of freeway through right most lane and left 
edge of exit ramp) is 0.5 meters, and ending at the exit gore where the two pavement edges are 3.7 meters apart.

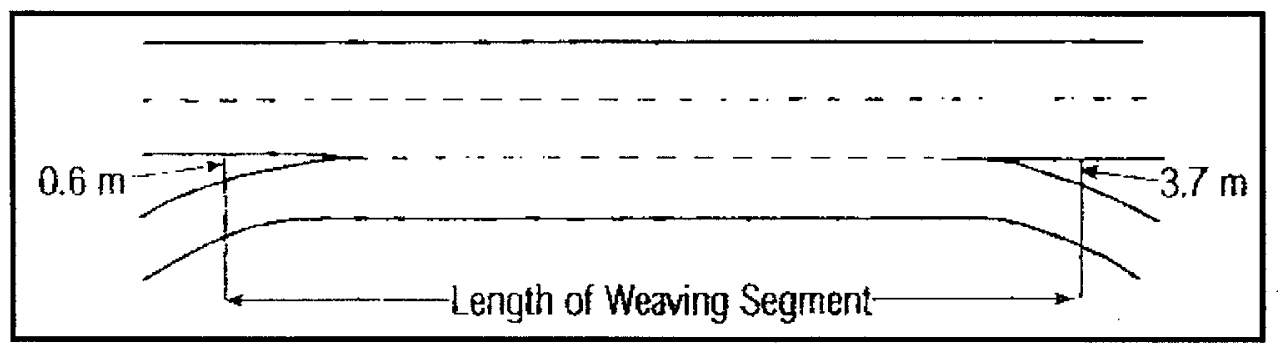

Figure 2.9: Length of Freeway Weaving Section (HCM 2000, pp. 13-18)

\subsection{REVIEW OF PREVIOUS RESEARCH}

After the brief review of the design guides that was presented in the previous section, this section extends to include the previous studies that addressed the effect of design criteria on driving behaviour specifically on freeway diverge areas. The main focus of these studies is the freeway deceleration SCLs. This section also provides a review that focuses on freeway safety performance at freeway diverge areas.

\subsubsection{Driving Characteristics on Freeway Diverge Areas}

In early 1950's, there have been debates on advantages and disadvantages of using the two main types of freeway off-ramps; namely: parallel lane and direct taper lane. Conklin (1959) compared the operating characteristics of the two types of ramps. Two locations were selected for the study on the R. H. Baldock freeway, a part of the Interstate Highway System between Salem and Portland. The use of portable radar units assisted in recording speeds at the beginning, center, and near the gore point of the off- 
ramps. The speeds of through vehicles in the outside lane of the freeway were also recorded for the study with a minimum of 100 vehicles sample. Moreover, the lateral placement at which vehicles cleared the outside lane of the freeway was also determined. Conklin (1959) concluded that the direct taper design is definitely superior to the parallel type in terms of vehicle operating characteristics. The reason was that a direct taper is more closely follows the path that most drivers select when approaching the ramp. Vehicles using the direct taper type off-ramp consistently leave the freeway at about the same location and the manner the SCL was designed. This indicates a smooth and more orderly movement of the traffic at the diverge area. Exiting vehicles were also observed of leaving the outside lane of the freeway at relatively high speeds, reducing possibilities of collision occurrence on these areas. In the case of the parallel type off-ramp, drivers used the SCL in different manners. It was concluded that vehicles exiting the freeway using the parallel type lanes did not follow a particular manner, rather varied from full use of the deceleration lane to almost no use of the lane. This indicates unsafe freeway areas with high collision potential.

Berry et al. (1963) studied the effect of different operational aspects on three lefthand exit ramps compared to a right-lane exit ramp on a six-lane section of the Congress Street Expressway in Chicago, Illinois. The research attempted to carry out detailed analyses of volume, speeds, density, existing paths, and hazardous manoeuvres to study the operational behaviour patterns of traffic at high-volume left-hand exit ramps. Many of the states interviewed for the study had designed extra length into left-hand deceleration lanes in an attempt to minimize possible safety issues. Based on the information gathered in this study, it was concluded that there were no appreciable 
increases in percentage of total volumes than at an approach to a right-lane exit ramp. Traffic speeds were not also affected by left-hand exit ramp design. In addition, no noticeable increases in density upstream left-hand exit ramps were found during the study. It was also concluded that no indications of hazardous manoeuvres were highlighted due to the locations of directional signing in advance of the required lane change manoeuvre.

Fukutome and Moskowitz (1963) studied traffic behaviour at off-ramp terminals on a number of California freeways. The data for the study were collected using Traffic Analyzer equipment furnished and operated by personnel of the U.S. Bureau of Public Roads. This equipment recorded the flow of traffic over the exit ramps. Freeway offramps in California are designed with a direct taper rather than the parallel deceleration lane. After it was observed that almost no drivers actually followed the reverse curve alignment required by the parallel design, the design standard was changed to use direct taper type off-ramps. However, several aspects such as reported accidents indicated that the deceleration distance associated with direct taper lanes were not adequate in many cases. Therefore, the study was conducted to determine whether the deceleration distance to the ramp curve past the curb nose is adequate and has any effect on operating speed of exiting vehicles. The researchers collected speed data at 8 existing off-ramps for studying the operating characteristics of the traffic as it leaves the freeway. It was concluded that in designing an off-ramp every effort should be made to provide a high-speed design keeping in mind not to provide geometrics that might attract through vehicles onto the off-ramp. However, the driver should be provided with sufficient length to decelerate to 
the speed of the terminal control. This length of the deceleration lane should be controlled by the ramp terminal curve.

The study that was carried out by Davis and Williams (1968) considered different operating characteristics such as headways, speeds, lateral placements, and deceleration rates on six various types of outer loop and diamond exit ramps located on Highway 401 in the Toronto area, Ontario, Canada. Vehicle movements of 1,574 vehicles were recorded on film for operational pattern analysis on deceleration lanes as well as the adjacent through lanes. Among the key findings of the research was that there is sufficient evidence that deceleration lanes are not being used as designed. This is primarily due to the fact that many vehicles do not enter the deceleration lane at its beginning. This may cause inconvenient interfering with drivers who desire to maintain full speed in the through lanes. It was suggested that a direct taper design at exit ramps would be preferable to possibly eliminate ant traffic conflicts. Davis and Williams (1968) concluded that the variation of vehicle's speed in diverge areas may influence even more than resulted by speeding drivers on a freeway basic section.

Pahl (1972) notes that there are specific driver-related problems that may be intensively observed at interchanges - particularly when drivers approach an off-ramp. In a study conducted in the Los Angeles area of California, photographic shots of vehicles upstream off-ramps were recorded. It was found that a driver approaching an off-ramp reacts in a different way than that on a freeway through lane. Typically, while drivers attempt to pass slower vehicles in the right lane, drivers would slow down to remain in contact with the right lane when they tend to exit the freeway. As the study admits, "such differences in lane-change characteristics of exiting vehicle drivers in the vicinity of their 
intended exit from those of through vehicle drivers may result in special operational and safety problems in the vicinity of off-ramps".

Another study conducted by Livneh et al. (1988) addressed vehicle behaviour at deceleration lanes. The focused on traffic behaviour at three rural deceleration lanes: one on a major freeway, the second on an expressway, and the third on a two-lane two-way highway. Paths of cars and trucks were recorded through continuous video photography of the traffic flow on selected deceleration lanes. Speed profiles were obtained for the recorded vehicles along both deceleration and through lanes. It was noticed in this study that the actual behaviour of drivers on deceleration lanes is not always consistent with the assumptions listed in the AASHTO design guide. The researchers based their conclusion on three primary observations: (1) the speeds of diverging vehicles at the beginning of the deceleration lane were lower than those for the through vehicles; (2) the deceleration values obtained were lower than values suggested by AASHTO; and (3) the average initial deceleration in gear lasted three times as long as the value (3-seconds) assumed for design purposes. The study findings led to the formulation of a deceleration lane length model that could be written as follow:

$$
L=7.75 \times S T+\frac{\left[(0.667 \times S T)^{2}-(S R)^{2}\right]}{2 d}
$$

Where, $L=$ length of deceleration lane $(\mathrm{m}) ; S T=$ average running speed of vehicles on freeway through lane $(\mathrm{m} / \mathrm{s}) ; S R=$ average running speed of vehicles on $\operatorname{ramp}(\mathrm{m} / \mathrm{s})$; and $d=$ deceleration rate while braking $\left(\mathrm{m} / \mathrm{s}^{2}\right)$.

Nevertheless, Livneh et al. (1988) stated that although the developed model for determining the length of a deceleration lane is based on limited data, it is preliminary in its nature. The developed model uses the average running speed on freeway and ramp as 
well as it does not require the determination of speed at the end of the braking (deceleration) behaviour on the deceleration lane.

In the study by Fazio et al. (1990), a general behavioural model of diverging from a freeway to an exit area was developed. Mathematically, the developed model defined three sequential response elements that together characterize the length of speed-change lanes. These three elements were reported as: (1) a criterion for a driver to diverge from a freeway (based on the angular velocity of the exit ramp gore), (2) the distance required for a driver to complete a steering-control manoeuvre on SCL, and finally (3) the distance at which a driver begins to brake to move from a SCL to a curve of an exit ramp. The researchers compared the distances predicted from the developed model to observed existing deceleration distances using video records of traffic on seven exit ramps and concluded that the model is a reasonable representation of the existing driving manoeuvres. The model offered a rational basis for deceleration speed-change lane design rather than the usual empirical means based on vehicle characteristics.

Harwood and Mason (1993) examined the relationship between ramp speeds and design considerations. This study focused on operational and safety issues that may result from ramp design speeds that are too low. The study concluded that the important factor for designers to consider is the relationship between ramp design speed and freeway design speed. An effort must always be made for these two speeds to be as close together as safely feasible.

Koepke (1993), compared between taper and parallel ramps using a survey of 45 states. It was mentioned that 4 states preferred the use of the parallel design, 11 used a taper design exclusively, and 30 states used a combination of both designs. However, 
those 30 states that use a combination of both designs use taper type exclusively for exit ramps and use parallel type for entrance ramps. These results would seem to indicate that the taper design style is highly preferred for exit ramps on nearly all freeways. However, Koepke (1993) noted that the gore of exit ramps appears to be the area where many freeway collisions occur. It was also mentioned that many drivers have problems with driver gap acceptance on freeways and in proximity to exit terminals. The conclusion was that a majority of drivers either does not know how to properly use the speed-change lanes or simply choose not to use them properly.

Al-Kaisy et al. (1999) also studied freeway diverge areas with an emphasis on capacity and operational performance. Their research discussed the use of computer traffic simulations to examine five variables related to the diverge area including (1) total upstream demand, (2) off-ramp demand, (3) length of deceleration lane, (4) off-ramp free-flow speed, and (5) number of lanes at mainline. Using these variables, the researchers considered key geometric characteristics and traffic conditions as they related to operational performance and highway capacity. The study used a typical section of a Canadian freeway. The findings indicated that, contrary to HCM (1994 and 1997) procedures, drivers' manoeuvres at diverge areas would significantly impact freeway capacity at these areas. Additionally, it was found that the proportion of diverge traffic had a more significant on performance during times of heavy to moderate traffic volumes.

Retting et al. (2000) discussed the effect that pavement marking has on speeds at freeway exits. In their study, the effect of narrowing the lane width of the curve along with a portion of the tangent section leading into the curve was examined. The narrowing 
of the lane was done by using a gradual inward taper of existing edge line. Speeds were measured six weeks prior and two weeks after installing the pavement marking that narrowed the lane width in the ramp approach. The authors focused on the changes that took place in mean speeds, $90^{\text {th }}$ percentile speeds, and percentage of passenger vehicles exceeding posted advisory speeds by more than specific values $(16.1 \mathrm{~km} / \mathrm{h}$ and $8 \mathrm{~km} / \mathrm{h})$. The study concluded that installing the marking resulted in a drop in the percentage of passenger vehicles traveling more than $16.1 \mathrm{~km} / \mathrm{h}$ above the posted speed by $6 \%, 13 \%$, and $17 \%$ for three sites out of the four sites examined. No results were gathered from the fourth site. Significantly, the proportion of large trucks found exceeding the posted speeds by $8 \mathrm{~km} / \mathrm{h}$ have decreased by $22 \%, 17 \%$, and $19 \%$ for the same three sites.

Liapis et al. (2001) studied operating speeds $\left(V_{85}\right)$ on curved sections of the ramp proper. The rate of change of curvature $(C C R)$, superelevation rate $(e)$, pavement width, width of paved shoulder, sight distance, grade configuration, and the operating speed data for twenty on and off-ramps in Greece were collected for that study. Liapis (2001) succeeded in developing two prediction models, one for on-ramps $\left(R^{2}=0.73\right)$ and other for off-ramps $\left(R^{2}=0.75\right)$. However, $C C R$ and the superelevation rate were the only two statistically significant parameters that explained the variability in the operating speeds on the collected sites. It was also concluded that speeds on on-ramps were higher than those on off-ramps by approximately $10 \mathrm{~km} / \mathrm{h}$ for the same $C C R$ and superelevation rate.

According to a study by Dutta et al. (2002), poor sign location and arrangement may be a contributing factor in some cases of poor driver performance on freeways. That study assessed the effect of the design of overhead signs on driver behaviour during weaving manoeuvres. The signs located on freeways in the area of Logan International 
Airport in Boston, Massachusetts were examined to determine their affect on safety. It was stated that poorly designed exit signs could lead to missing the desired exit or making a decision at the last moment to switch lanes, leading to potentially unsafe manoeuvres. Problems were found with the existing signs, including non-centered positioning over lanes, which lead to directional arrows pointing at the improper lane. Using a simulation, Dutta et al. (2002) tested the performance of drivers with four different types of overhead signs. In general, the study recommended that better performance could be achieved on weaving sections by using more clear and understandable signs.

Garcia and Romero (2005) studied the behaviour of vehicles on deceleration lanes of various lengths in Valencia, Spain. The study proposed a kinematic model for deceleration lanes of tapered type. The model is based on the behaviour of passenger cars since heavy trucks need longer distances to decelerate. However, longer deceleration lanes are not recommended since average speed for heavy vehicles are lower than those for passenger cars. The researchers suggested that a longer deceleration could actually impact safety since it would provide too much distance for deceleration allowing following vehicles to overtake a preceding vehicle on the lane. It was stated that exiting freeways is carried out based on two manoeuvres, (1) lane change with deceleration and (2) braking. The study found that if deceleration lanes are too long, an acceleration manoeuvre may become necessary, and if it is too short, the lane change manoeuvre occurs within the braking deceleration. In conclusion, they found the length that balances the effects on the main roadway with the best functionality and safety of the deceleration lane is the one that allows successful joining of the two manoeuvres. 


\subsubsection{Safety Performance}

According to Harwood (1995), who studied geometric design improvements, there is little coordination between operational improvements and safety considerations in most freeway design. The study noted that, "in the real world of highway design, there is a clear need for operational analysts and geometric designers to be familiar with existing accident patterns at a site and the likely safety performance of candidate alternative solutions". The overall recommendation of the study was to encourage producing geometric design policies that fit the needs of each specific roadway, which may require making exceptions to currently accepted standards.

Worldwide, many researchers have dedicated their research work to develop models that are capable of estimating individual consistency measures for design consistency evaluations and safety concerns on two-lane highways. In fact, less consistency and safety work has been conducted for freeways. Nevertheless, following are the relevant research work related to freeway deceleration SCLs and diverge areas.

In one of the earliest studies of its kind, Lundy (1965) studied 722 freeway ramps being used by a total of two billion vehicles. It was discovered that $32 \%$ of the ramps had experienced no collisions at all, while a total of 1643 collisions were experienced on the remainder of the ramps. The ramps considered for the study experienced $18 \%$ of the total collisions on the freeways they were connected with. Lundy's study was designed to classify the geometric features of ramp types and examine the relationship between each type and related safety issues. The researcher concluded that there was no statistically significant difference between ramps that had experienced collisions and those that experienced no collisions. As has been the case in many other studies under review, it 
was determined that exit ramps have a much higher rate of accidents than entrance ramps. Recommendations included using an acceleration lane length of $243 \mathrm{~m}(800 \mathrm{ft})$ or greater and a deceleration lane length of $274 \mathrm{~m}(900 \mathrm{ft})$ in order to increase the safety in these areas. In spite of this, there were two limitations evident in this study. First, collisions were assumed to be related to traffic volumes only, regardless of the length of the ramp. Second, using the collision rate has recently been considered unsatisfactory since it is based on the false assumption of linear relationship between number of collisions and traffic volumes.

Cirillo (1970) investigated the relationship between the geometry of an interstate highway system and safety. This study included the effect of weaving length, acceleration and deceleration lanes on safety performance for specific segments. The study examined the accident rate per hundred million vehicles using accident data from 2,288 highway sections, collected during 1961-1965 in twenty US states. It was concluded that increasing the length of weaving sections is recommended to increase safety, specifically by reducing collision rates. Therefore, additional length of both acceleration and deceleration speed-change lanes would be beneficial to reduce the collision rates for these areas. Nevertheless, the increased length of acceleration lanes appeared to produce a greater sensitivity of safety improvement than increasing the length of deceleration lanes. This is primarily due to the fact that deceleration lanes are generally safer than acceleration lanes regardless of the length of lanes or extent of traffic flow on diverge or merge area.

Harwood and Mason (1993) stated that AASHTO policies for freeway ramp design are adequate to maintain a high level of safety, as long as drivers regulate their 
speed to levels less than or equal to the design speed. For vehicles, especially trucks, travelling with higher speed than the design speed on ramp curves, safety problems are more likely to arise. The study reported that the most critical conditions may occur on horizontal curves with lower design speeds of 20 to $30 \mathrm{mph}$ ( 32 to $48 \mathrm{~km} / \mathrm{h}$ ). A truck on a curve with a $20 \mathrm{mph}(32 \mathrm{~km} / \mathrm{h})$ design speed has a very small margin of error since it can rollover when travelling at or with only $25 \mathrm{mph}(40 \mathrm{~km} / \mathrm{h})$ and may skid off the road under serious wet-pavement conditions when travelling at about $27 \mathrm{mph}(43 \mathrm{~km} / \mathrm{h})$. Additionally, a truck on a ramp curve with a $30 \mathrm{mph}(48 \mathrm{~km} / \mathrm{h})$ design speed can experience a rollover at a speed of about $38 \mathrm{mph}(61 \mathrm{~km} / \mathrm{h})$. Harwood and Mason (1993) further recommended that the design policy for horizontal curves should ensure an adequate safety margin in an attempt to prevent both rollover and skidding at the actual speeds used by vehicles in reality. In other words, the design of the ramp curve should consider how fast drivers will actually be travelling on the ramp, and not simply be an exercise in design for its own sake.

Models for predicting accident rates using 1988 and 1989 data were developed by Persaud and Dzbik (1993). The macroscopic model in this study used annual accident rates per kilometre in addition to the average daily traffic volumes data from nearly 500 segments of freeways in Ontario, Canada. The researchers determined that there was a lower risk for collisions on freeways consisting of four lanes as opposed to those with more than four lanes. The higher risk for collisions on the larger freeways was due higher volumes of traffic at rush hour in urban areas.

Twomey et al. (1993) conducted a study on the safety of interchange design features, reviewed in terms of accident rates per 100 million vehicles. This research 
indicated that by providing an enhanced geometric design to both entrance and exit terminals, safety could be increased. The recommendation was for auxiliary or acceleration lanes to have a minimum length of $800 \mathrm{ft}(244 \mathrm{~m})$ and deceleration lanes to be $900 \mathrm{ft}(274 \mathrm{~m})$ in length. Lengthening these lanes was only one step that the study concluded to improve the safety of freeway operation. Other factors also included adding ramp lanes, optimizing existing ramp lanes or installing new traffic signals, adding collector-distributor roads and lengthening weave areas.

Bauer and Harwood (1997) evaluated the relationship between traffic accidents, geometric design features and volume of traffic through development of several statistically significant models at 10\%-20\% significance level. These models included interchange ramps as well as SCLs with $R^{2}$ of 0.10 to 0.42 . The 3 -year traffic collision data on 551 ramps in Washington State (US) from 1993 to 1995 were used in the modelling attempts using the negative binomial regression technique. The models showed that the variability in the collision data were explained by different parameters such as ramp average annual daily traffic ( $A A D T)$, ramp type (rural or urban), ramp configuration (exit or entrance), and combined length of the ramp and the speed-change lane (including taper). The developed models showed that the independent variables explained $10 \%$ to $42 \%$ of the variability in the collision data. It was noted that about between $50 \%$ and $80 \%$ of the ramps have experienced no collisions or only one collision. The study used the taper length as well as the length of the SCL in the analysis, as a lack of necessary data in the design guides on how to determine the SCL length. It was concluded that modelling the total number of collisions occurred on ramps in addition to 
the adjacent SCL were more significant than those resulted from disaggregating the collision data into two separate parts.

Awad and Janson (1998) studied the prediction of commercial truck collisions on freeway ramps in the Washington State. All collisions that took place on ramps, acceleration/deceleration lanes, or in the lanes adjacent to the acceleration/deceleration lanes were included in this study. In the study, 82 out of the total 391 interchanges were examined and have experienced no collisions at all during the study period of the study. The analysis encompassed four ramp categories; namely: diamond, loop, outer connector, and directional. Additionally, a dataset that contained records of 1,030 truck collisions, which occurred at 581 ramps, was used to model truck collisions. The research revealed a total of ten factors that accounted for more than $75 \%$ of the total variation in the collision data. However, out of those ten factors, only four were found to be sufficiently relevant to the study. These four factors were (1) the distance between the gore and the taper, (2) volume of traffic on ramps, (3) volume of traffic on through road, and (4) the percentage of truck traffic on the main road. Other factors that were considered insignificant included ramp length, truck percentage on the ramp, weather conditions, urban/rural location, visibility, and road surface conditions. Either Poisson or negative binomial regression was favoured in the study rather than the traditional linear regression modelling.

Khorashadi (1998) studied the relationships between ramp configuration, ramp type, ramp geometry, and collisions using a three-year collision data retrieved from 13,325 ramps on the California State Highways. This study found no statistically significant difference in collision rates between different types of ramps. The most 
common areas to experience high collision rates per million vehicles were scissor ramps, rest area ramps, and slip ramps. Rural ramps were notably safer, accounting for only $2.82 \%$ of the collisions occurring on rural highways. On the other hand, urban ramps accounted for $18.41 \%$ of the collisions occurring on the urban highways.

Bared et al. (1999) studied safety and economic evaluation techniques related to acceleration and deceleration lanes in the Washington State. The study used previously developed collision models (developed based on a sample of 276 exit ramps and 192 entrance ramps) to help highway planners and designers assess safety improvement benefits for interchange ramps and the adjacent speed-change lane. Using previously reported statistical relationship between collision frequency and speed-change lane length, a benefit/cost analysis were conducted to evaluate the effect of increasing the length of speed-change lanes to achieve an economically acceptable design. Considering interchanges for reconstruction or when they are experiencing higher collision frequencies, the proposed methodology is a useful tool for safety evaluation especially when existing collision records are not required. It was recommended that lengthening deceleration lanes in particular would be more beneficial, as they experience a higher number of collisions than the acceleration lanes.

Khan et al. (1999) developed collision models for urban and rural segments separately using ten-year collision data on an interstate highway I-25 (between Denver and Pueblo) in Colorado, US. The collision data included injury, property damage, and total collisions of 97 segments of Highway I-15 covered a portion of 160 miles $(258 \mathrm{~km})$. The developed models used Poisson regression with log-transformed parameters for two different sets of variables (1) segment length (SL) and average daily traffic (ADT) and (2) 
vehicle miles traveled $(V M T)$. It was established that both $S L$ and $A D T$ were more significant in predicting injury and damage collisions, while the $V M T$ was more significant for predicting fatal collisions only.

Golob and Recker (2003) used linear and nonlinear multivariate statistical analysis to determine how the various types of freeway collisions are related to weather, volume of traffic, and lighting condition. Six freeways in Southern California were used as the basis of the study. The data collected from nearly 1200 vehicle collisions during the year 1998 were used in the research. The collisions were grouped according to the type and location of the collision, number of vehicles involved, and collision severity (in terms of injury versus property damage only).The study stated that wet road surfaces were more likely to produce collisions involving vehicles hitting a side-object as well as collisions involving multiple vehicles. However, these same kinds of collisions were also found to be related to relatively stable traffic flow characterized by low volumes and high steady speeds. It was interesting that the study also found that rear-end collisions were more likely to occur on dry roads during daylight hours, where there is a high disparity in relatively low speeds. The primary factor influencing collision severity, according to Golob and Recker (2003), is the volume of traffic. In general, the higher the traffic flow, the greater the risk for severe collisions.

Kockelman and Ma (2004) examined average speed and speed variation patterns and their relationship with collision occurrence. Six orange country freeways in California were used to collect data upstream and within 2,000 $\mathrm{ft}(610 \mathrm{~m})$ of the collision locations using single loop detectors. The study concluded that speed has no significant influence on collision occurrences on freeways. 
McCartt et al. (2004) examined a sample of 1,150 crashes that occurred on 176 of heavily traveled urban interstate ramps in Virginia. Nearly half of these collisions occurred when at-fault drivers were exiting the freeway while $36 \%$ occurred when drivers were entering the freeways. Additionally, only six percent of collisions occurred on midpoint of access roads or on ramps connecting two separate freeways. Research concluded that the crash type most frequently associated with exiting was run-off-road, while the crashes most common with vehicles entering the freeway were rear-end or sideswipe/cutoff types. Suggestions to enhance safety performance included increasing the ramp design speeds, extension of the acceleration lanes and enforcement of existing laws.

Sarhan (2004) developed several models for the prediction of five-year collisions using data from 94 segments and 26 interchanges of Highway 417 located in the City of Ottawa, Ontario. The study objective was to quantify the effect of ramp terminal spacing and traffic volumes on safety performance of freeways through regression analysis. Negative binomial regression was found to better fit the collision data over the Poisson regression. The study resulted in 24 statistically significant models at $95 \%$ confidence level. Several explanatory variables were examined including traffic volume, traffic exposure, type and length of speed-change lane, number of lanes, weaving types, and advisory speed on exit ramps. Sarhan (2004) recommended the use of two specific significant models, out of the 24 models developed, as useful ones in improving the safety performance of freeway merge and diverge areas. These two models had a unique characteristic that the explanatory variables explained the variability in the dependant variables and appeared in the models are design elements that can be controlled for safer 
operational conditions on freeways. The model for predicting the number of collision on diverge areas was given as follows:

$T C o l=e^{(2.7238+0.0136(\text { Expo })-0.0015(L D e c)+1.2143(\text { DecCo })}$

Where, $T C o l=$ Total five-year collisions for a freeway segment including the diverge area $;$ Expo $=$ Traffic Exposure in million-vehicle-km of travel; $L D e c=$ Length of deceleration lane in meters; $\mathrm{Dec} C \mathrm{O}=$ Code of deceleration lane $(0$ for limited length type SCL and 1 for extended length type SCL).

Additionally, Sarhan (2004) concluded that increasing the number of exiting vehicles at a specific freeway segment would cause number of collisions on this segment to increase. Additionally, the number of collision is expected to increase while carrying the full width of the SCL from an entrance terminal to the following exit terminal. The study recommended use of tapered portion at the beginning of a deceleration SCL, and did not recommend extending the SCL to the next ramp in cases where the basic number of lanes is to be increased or decreased.

Maze et al. (2005) studied the characteristics of safety performance on expressway segments near at-grade intersections using a three-year collision data (19992001 in Minnesota and 1998-2000 in Iowa). The study developed two statistical models for Minnesota and Iowa State Expressways. It was concluded that safety is highly affected by traffic volumes $(A D T)$ and crash density. The researchers recommended the use of grade separation with full access control for safety benefits on high-volume expressway segments, as safety found to deteriorate with increasing the traffic volumes. 


\subsection{SUMMARY}

Based on the information presented in this chapter that addressed the effect of different design criteria on driving behaviour at freeway diverge areas and SCLs, it is evident that inconsistency in some design criteria existed. Such inconsistent design criteria would not properly represent current driving behaviours on freeways. To date, relatively little research has been given to the synchronization and effect of geometric design elements of freeway diverge areas on driver behaviour. Therefore, this study is intended to examine and model driver speeds and deceleration behaviours on deceleration SCLs using geometric features and traffic volume. Moreover, this study will attempt to model safety performance of freeway deceleration SCLs using related speed, geometric, traffic, and collision data. It is hoped that the models to be developed in this study, if any, would be in assistance for highway designers in selecting the proper geometry of deceleration SCLs for efficient and safe operation of freeway diverge areas. 


\section{CHAPTER THREE \\ 3.0 DATA COLLECTION AND PREPARATION}

This chapter covers a detailed description of the database collected and prepared for the purpose of this study. Based on the study objectives and the literature review presented in previous chapters, proper data have been collected and prepared for developing operational and safety performance models that would help evaluate and predict drivers' behaviour on deceleration speed-change lanes (SCLs) and the adjacent right lanes of freeways. The data included operating speeds, traffic counts, freeway geometric, and collision data for candidate sites selected for this study. The speed data were collected from deceleration SCLs and the adjacent right most through lanes on Highway 417 in Ontario, Canada. Two research students had worked together in the speed data collection process for the purpose of this study and another concurrent research study (Ahammed, 2005).

Section 3.1 presents the study area and the sites selected with the relevant constraints behind this selection. Then, Section 3.2 provides a description of the equipment used for collecting the speed and traffic data. The speed data collected as well as the preparation of different speed and deceleration parameters (driving behaviour measures) useful for the modelling procedures are presented in Section 3.3. Then, Section 3.4 describes the geometric plans used to extract the needed geometric data for the selected sites, and Section 3.5 presents the traffic data preparation. Finally, Section 3.6 presents the collision data used for safety modelling activities. 


\subsection{SITE SELECTION}

This section describes the proposed study area, site investigation process, and finally the sites selected for the purpose of this study. As previously mentioned, the main objective of this study is to develop operational and safety performance prediction models to help evaluate drivers' behaviour on freeway deceleration SCLs and the adjacent right lanes. Therefore, a suitable and acceptable database with adequate numbers of observations is necessary to accomplish the study goals.

\subsubsection{Study Area}

The study area included in this research covered a long portion of Highway 417, also known as the Queensway. Highway 417 is a major freeway in Ontario, Canada that extends from Route 40 (the Quebec border) in the east to Highway 17 (Amprior town) in the west. The selected study area covers the section from Interchange 110 (intersection with Walkley Road) east to Interchange 145 (intersection with Highway 7) west. Queensway is the only freeway running through the City of Ottawa, Ontario, Canada, serving a high level of commuter traffic with a speed limit of $100 \mathrm{~km} / \mathrm{h}$. Under favourable traffic conditions, drivers are more likely to select speeds close to or above the Queensway's speed limit, whereas during the rush hours periods, congestion builds up and drivers are forced to reduce their operating speed.

Shown in Figure 3.1 is the selected study area that covers two distinctive land-use types located within the City of Ottawa. The Eastern and Western regions of the study area are classified as urban commercial and industrial areas, while the central region is classified as central business district (CBD) area. Moreover, the central region is 
associated with higher numbers of interchanges and limited right-of-way, serving the city core, resulting in shorter spaces between the successive ramp terminals in both directions of the freeway (i.e. shorter SCLs). Furthermore, the traffic along the Queensway varied from one interchange to another depending on the type of the land-use being served.

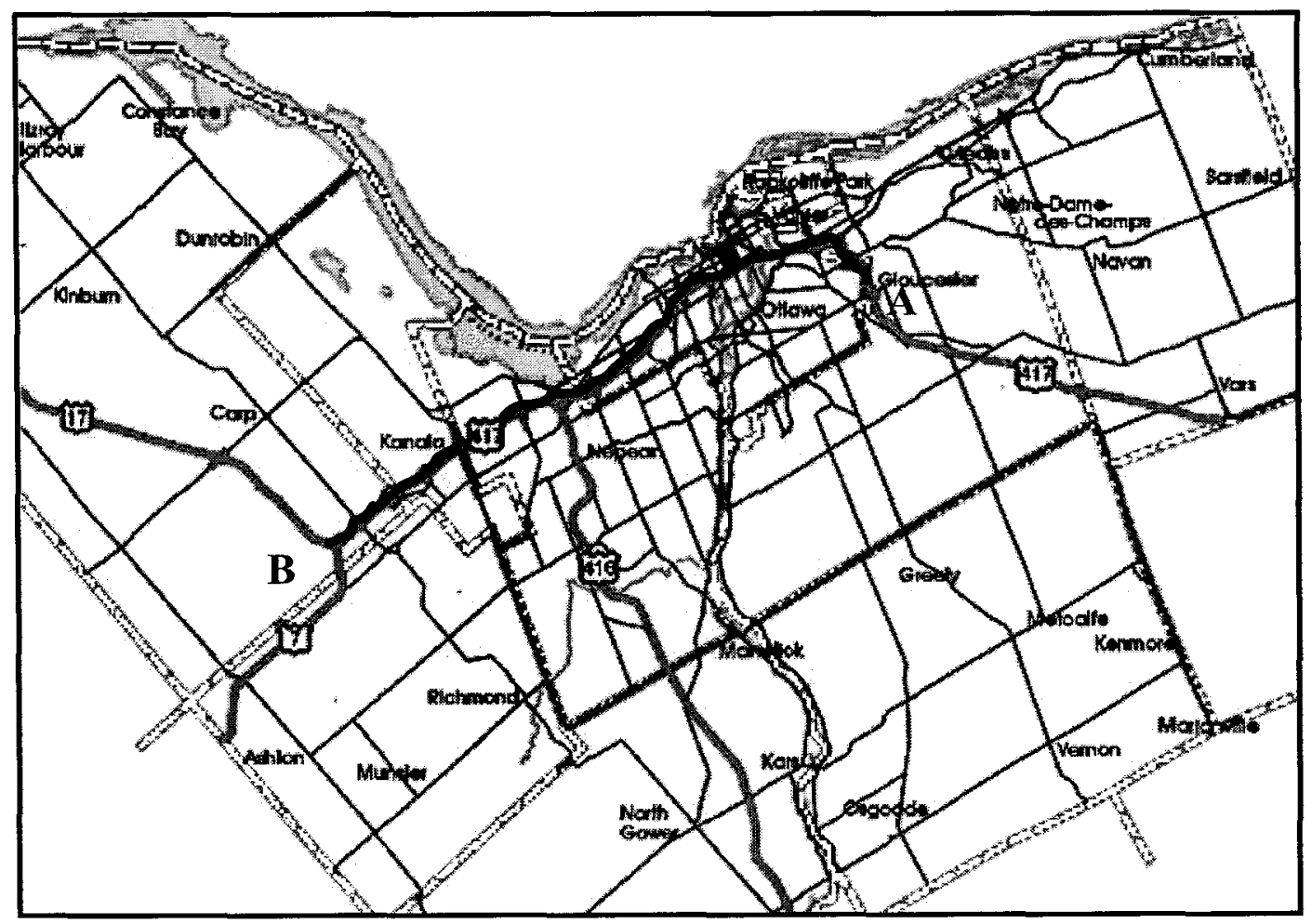

Figure 3.1: Selected Study Area of Highway 417

The selected study area was found to exhibit a noticeable variability in the geometric and traffic data from one interchange to another along the freeway. Such variability should help reveal any existing effects on drivers' behaviour on deceleration SCLs and the adjacent right lanes of freeway diverge areas from operational and safety performance points of view. 


\subsubsection{Site Investigation}

After visiting the proposed study area, 27 interchanges with 43 exit ramp terminals, in both directions of the freeway, were available for study including the newly constructed interchange (Castlefrank Road - IC\# 139). The section of the Queensway, between Eagleson Road (IC\# 110) and Terry Fox Drive (IC\# 140) interchanges, where the Castlefrank Road is located, was designated as a construction area but open for public use. During the speed data collection process, this section of the freeway was still under the responsibility of Morrison Hersfield - a multidisciplinary engineering and management firm. Therefore, Morrison Hersfield were consulted regarding their permission for use of the Castlefrank overpass as well as the pedestrian overpass, that was reconstructed near the Castlefrank interchange.

Excluding freeway-to-freeway exit ramps, 40 exit ramp terminals were the candidate sites to select amongst them the suitable locations for speed data collection. The selection of the appropriate locations for the speed data collection process from the Queensway was controlled by the following key constraints:

- Operator's visibility: Locations with limited visibility of deceleration SCLs and the adjacent right lanes to the operators were excluded.

- Driver's distraction: Locations where the operator would be visible to the drivers and thus could affect their driving behaviour were excluded.

- Operator's safety: Locations that represented over due hazard to the operators were excluded.

Based on those constraints, four possible alternative equipment (operator) positions were established. These four possible locations were: (1) an overpass of an 
interchanges, (2) a pedestrian overpass, (3) roofs or upper floors with side windows of elevated buildings adjacent to the Queensway, or (4) nearby mall parking areas. Subsequently, 27 exit ramp terminals were excluded from the study area due to the following reasons:

- The site has not satisfied any of the four alternatives.

- Property owners of the buildings, adjacent to the freeway that were chosen for operators' positions, refused to authorize the use of their property for speed data collection activities.

- The traffic volume on the exit ramp was less than five veh/h.

Finally, the site investigation process resulted in 13 exit ramp terminals at 11 interchanges. The following sub-section (Section 3.1.3) describes the sites selected for the purpose of this study. Since the Queensway is a provincial property, the Ontario Provincial Police (OPP) patrols it. All other local streets of the City of Ottawa, Ontario (including freeway overpasses) are under the jurisdiction of the Ottawa Police. For that reason, both the OPP and the Ottawa Police were informed about the dates, times and the equipment used regarding the speed data collection activities. In addition, the Ministry of Transportation (MTO) and the OPP were updated on a weekly basis of the schedule of the speed data collection process, while the Ottawa Police were updated on a daily basis before heading to the designated site(s).

\subsubsection{Selected Sites}

With regard to the information previously mentioned, 13 exit ramp terminals at 11 different interchanges were selected and used for collecting actual speed data for the 
purpose of this study. The speed data from 12 exit ramp terminals (deceleration SCLs and the adjacent right lanes) were collected using the interchange overpasses, while the speed data for the Bronson W-NS exit ramp terminal were collected from the roof of an Ottawa-Carleton School Board building located next to the Queensway. Table 3.1 provides a summary of the sites selected for the purpose of this study. As shown in the table, seven sites were classified as limited length type SCLs (parallel or taper length type), while the other six sites were classified as auxiliary deceleration SCLs (extended length type). In addition, there were eight sites located on the eastbound direction while five on westbound direction.

Table 3.1: Summary of Selected Sites

\begin{tabular}{|c|c|c|c|}
\hline$\#$ & SCL Name & Equipment/Operator Position & SCL Type \\
\hline 1 & St. Laurent Blvd (W-NS) & Belfast Rd Overpass & Extended (Dual-Exit) \\
\hline 2 & Vanier Pkwy (W-NS) & Vanier Pkwy Overpass & Extended (Dual-Exit) \\
\hline 3 & Woodroffe Ave (E-NS) & Woodroffe Ave Overpass & Extended (Single-Exit) \\
\hline 4 & Woodroffe Ave (W-NS) & Woodroffe Ave Overpass & Extended (Single-Exit) \\
\hline 5 & Greenbank/Pinecrest Rd (E-NS) & Greenbank/Pinecrest Rd Overpass & Extended (Dual-Exit) \\
\hline 6 & Parkdale Ave (W-NS) & Parkdale Pedestrian Overpass & Limited (Taper) \\
\hline 7 & Prom Island Park Dr (E-N) & Parkdale Pedestrian Overpass & Limited (Taper) \\
\hline 8 & Terry Fox Dr (E-NS) & Castlefrank Rd Overpass & Limited (Parallel) \\
\hline 9 & Moodie Dr (W-NS) & Moodie Dr Overpass & Limited (Parallel) \\
\hline 10 & Terry Fox Dr (W-NS) & Terry Fox Dr Overpass & Limited (Parallel) \\
\hline 11 & Ch. Carp Rd (E-NS) & Ch. Carp Rd Overpass & Limited (Parallel) \\
\hline 12 & Eagleson Rd (W-NS) & Castlefrank Pedestrian Overpass & Extended (Single-Exit) \\
\hline 13 & Bronson Ave (W-NS) & Ottawa-Carleton School Board Building & Limited (Taper) \\
\hline
\end{tabular}




\subsection{FIELD SCHEME AND EQUIPMENT USED}

After the selection of the 13 exit-ramp sites for collecting actual (operating) speed data and prevailing traffic data on freeway deceleration SCLs and the adjacent right lanes, it was important to select the appropriate equipment that can be used to achieve the study goals. The equipment used for the data collection process in the field included the following:

1) SpeedLaser ${ }^{\circledR}$ Equipment

2) Video Camera

3) Laptop Computer

4) $\operatorname{Tripod}(\mathrm{s})$

5) Measuring Tape

6) GPS Unit

7) Reflective Safety Jackets and Traffic Cones

The SpeedLaser ${ }^{\circledR}$ is a laser-based speed gun, manufactured by Laser Atlanta, which has several advantages including the ability to precisely target and record continuous speed profile and/or speed values of a single vehicle on busy roadways. The foremost advantage of SpeedLaser ${ }^{\circledR}$ as compared to a radar gun is that the SpeedLaser ${ }^{\circledR}$ is capable of measuring and recording speed, distance, and time values of a target vehicle on a roadway as it travels along its path. The SpeedLaser ${ }^{\circledR}$ calculates the time it takes a pulse of a Class 1 eye-safe laser light to travel from the equipment position to the target vehicle for determining the relative distance (i.e. the vehicle's position from the operator). Hence, the equipment combines individual distance and time measurements to 
determine relative speed measurements. The SpeedLaser ${ }^{\circledR}$ captures data point readings (speed, distance, and time) and records these readings on a PCMCIA type II SRAM card.

Moreover, SpeedLaser ${ }^{\circledR}$ has the advantage of recording the speed of both approaching (oncoming) and receding targets and can easily differentiate between the two types by means of \pm sign, displayed next to the speed values on the rear panel display. A negative sign means that the target is receding away from the operator's position, while a positive sign means that the target is approaching the operator. Furthermore, the use of the monocular accessory facilitates the operator's operation of the device by improving the coordination between his/her eye-focus and hand-stability while aiming the laser beam on the target vehicle. Figure 3.2 shows an image of the SpeedLaser ${ }^{\circledR}$ used and the associated accessories. Moreover, Table 3.2 provides a summary of the technical specifications of SpeedLaser ${ }^{\circledR}$ equipment.

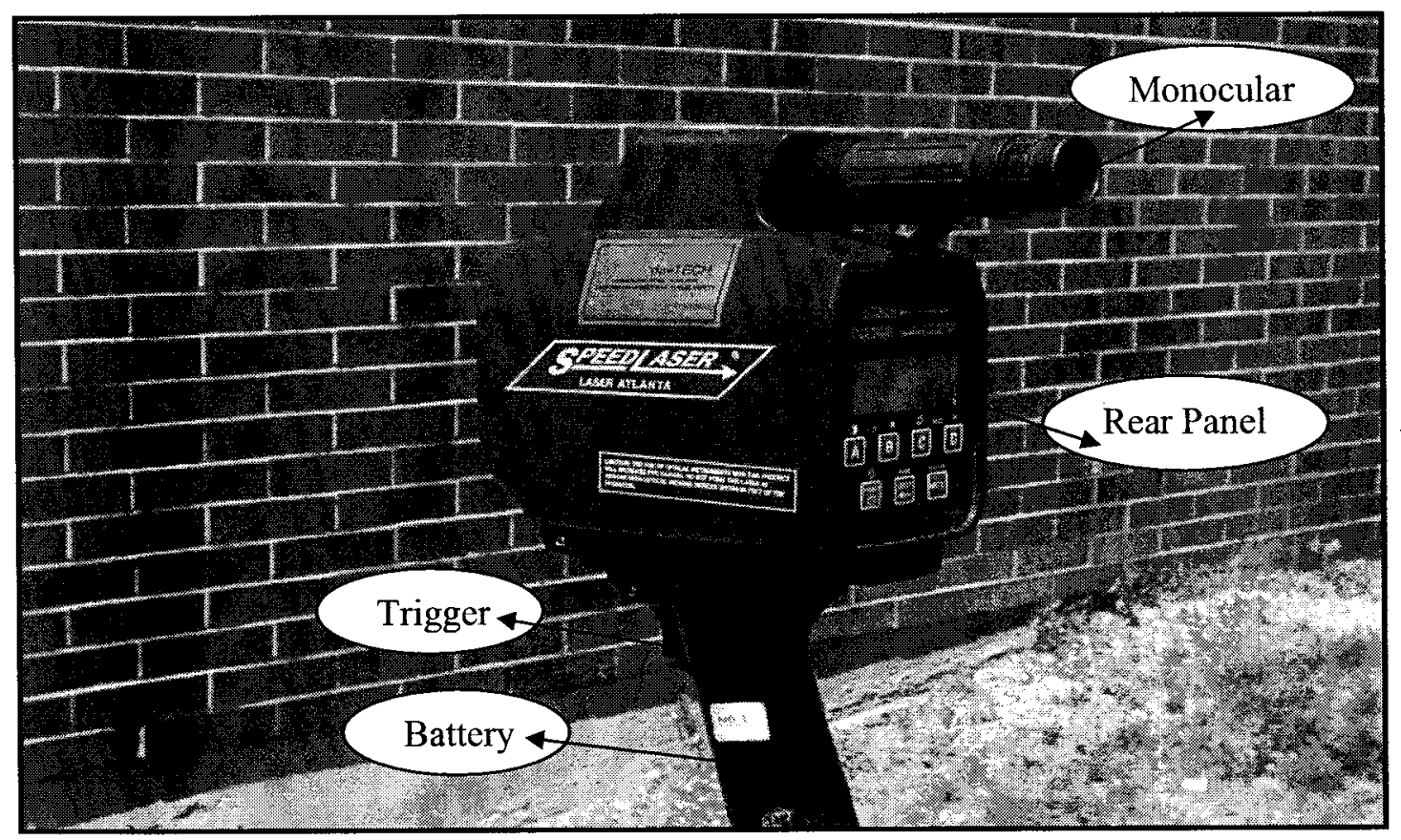

Figure 3.2: SpeedLaser® and Accessories, Laser Atlanta 
Table 3.2: Technical Specifications of SpeedLaser®

\begin{tabular}{|c|c|}
\hline Dimensions & $\mathrm{w} 11.5 \mathrm{x} \mathrm{h} 19.0 \mathrm{x} \mathrm{d} 21.5 \mathrm{~cm}$ \\
\hline Weight & $2.2 \mathrm{~kg}$, with battery \\
\hline Laser Classification & Class 1 eye-safe \\
\hline Speed Range & Maximum of $1,220 \mathrm{~m}$ \\
\hline Speed Accuracy & $\pm 1.6 \mathrm{~km} / \mathrm{h}$ \\
\hline Speed Measurement Limits & $16 \mathrm{~km} / \mathrm{h}$ to $320 \mathrm{~km} / \mathrm{h}$ \\
\hline Distance Range & $1.5-9,146 \mathrm{~m}$ \\
\hline Distance Accuracy & $\pm 0.3 \mathrm{~m}$ \\
\hline Data Capture & PCMCIA SRAM Type 2 card and slot \\
\hline Operating Temperature & $-30^{\circ} \mathrm{C}$ to $60^{\circ} \mathrm{C}$ \\
\hline
\end{tabular}

Two SpeedLaser ${ }^{\circledR}$ devices were used in the field to record operating speed data for vehicles diverging onto the freeway deceleration SCLs, in addition to vehicles travelling on the freeway adjacent right most through lanes. Hence, two operators worked together (side-by-side) to collect such vehicular speed profiles data on freeway deceleration SCLs and the adjacent right lanes.

On site, three tripods were used to support the two SpeedLaser $®$ devices to record speed data and a video camera for recording the traffic volumes on freeway diverge areas. While collecting the speed data, the PCM card sometimes reached its full capacity depending on the data recorded. When the PCM card got full, a laptop computer was used to transfer and store the recorded data. In addition, traffic cones were placed between the outer edge of the local traffic lane and the curb/sidewalk edge on overpasses with narrow sidewalk areas, to heighten the alertness of the motorists on the overpass.

Part of the field scheme was to determine the horizontal and vertical offsets of the equipment position with respect to the centerline of the individual SCL or the adjacent right lane designated for collecting the speed data. The horizontal offsets, measured on the overpass surface from the equipment position to the centerline of the respective SCL 
or the right lane, were determined by means of a measuring tape. On the other hand, the vertical offset values, measured from the pavement surface to the mid-point between the two front lenses of the SpeedLaser ${ }^{\circledR}$, were determined using both the SpeedLaser ${ }^{\circledR}$ and the measuring tape. First, the distance from the pavement surface to the top edge of the overpass fence was measured using the SpeedLaser ${ }^{\circledR}$. Then the distance from the top edge of the overpass fence to the mid-point between the equipment's two lenses was measured using the measuring tape. However, the vertical offset from the pavement surface to the top of the roof of the Ottawa-Carleton School Board building, and the vertical offset from the pavement surface to the top of the bridge deck at Parkdale pedestrian overpass, were both determined using a GPS unit. Whereas, the vertical offset between the pavement surface and the top of the bridge deck at Castlefrank pedestrian overpass was obtained from Morrison Hersfield. It should be noted that many of the horizontal offset values associated with the selected sites resulted in zero values, where the equipment position is exactly located on the centerline of the targeted lane.

As previously mentioned, two operators were collecting speed data for vehicles on the freeway deceleration SCLs and adjacent right lanes from the thirteen sites selected for this study. At each individual site, the first operator recorded speed data on the deceleration SCL while the other operator recorded speed data on the adjacent right lane. The speed data collection process involved targeting several vehicle movements with respect to the occupied lane on the freeway diverge area. For this purpose, a worksheet was prepared prior to the speed data collection process to manually record different vehicle movements of randomly targeted vehicles in the field. In addition, the worksheet was used to record all the information associated with the relevant site like the lane 
description, the horizontal and vertical offset measurements, and the vehicle classification. Table 3.3 shows a sample worksheet for Greenbank (E-NS) with examples of all possible vehicle movements expected to occur on a freeway diverge area with an extended (dual exit) deceleration SCL.

Table 3.3: Worksheet Sample for Recording Vehicles' Class and Movement

\begin{tabular}{|c|c|c|c|c|c|}
\hline \multirow{4}{*}{\multicolumn{3}{|c|}{$\begin{array}{l}\text { DATE: } \text { Oct. } 27 \mathrm{th} \\
\text { LANE DESCRIPTION: (E-NS) Extended, Dual } \\
\text { OPTERATOR POSITION: Greenbank Overpass } \\
\text { HORIZONTAL OFFSET: } \underline{\mathrm{SCL}=5.2, \mathrm{RL}=0}\end{array}$}} & \multicolumn{3}{|l|}{ IC\#: 129} \\
\hline & & & \multicolumn{3}{|c|}{ IC $\overline{N A M E}$ : Greenbank/Pinecrest Rd } \\
\hline & & & \multicolumn{3}{|c|}{ HWY DIRECTION: Westbound } \\
\hline & & & \multicolumn{3}{|c|}{ VERTICAL OFFSET: $\underline{\mathrm{SCL}=8.19, \mathrm{RL}=8.33}$} \\
\hline \multicolumn{3}{|c|}{ SPEED CHANGE LANE (SCL) } & \multicolumn{3}{|c|}{ RIGHT LANE (RL) } \\
\hline File \# & Vehicle Movement & $\mathrm{HV}$ & File \# & Vehicle Movement & $\mathrm{HV}$ \\
\hline 001 (Start) & & & 001 (Start) & & $\checkmark$ \\
\hline 007 & $\mathrm{D}$ & & 003 & ML & $\checkmark$ \\
\hline 032 & & $\checkmark$ & 004 & Ex & \\
\hline 044 & ML & & 014 & $\mathrm{D}$ & \\
\hline 062 & $\mathrm{D}$ & $\checkmark$ & 080 & $\mathrm{D}$ & $\checkmark$ \\
\hline 082 & Out & & 087 & Out & \\
\hline 135 (End) & & & 155 (End) & & \\
\hline \multicolumn{6}{|c|}{$\begin{array}{l}\text { HV = Heavy Vehicle } \\
\mathrm{D}=\text { Diverged from RL onto SCL } \\
\text { ML = Already Occupying SCL and Merged Left onto Inner Lane } \\
\text { Blank = SCL - Already Occupying SCL \& Depart; } R L \text { - Continued Through on Right-Lane } \\
\text { Ex = Exit onto Left-hand Ramp Lane of two-lane off-ramp from RL (Weaving Type B only) } \\
\text { Out = Corrupted File - Target Vehicle Lost }\end{array}$} \\
\hline
\end{tabular}

The vehicle movements associated with an auxiliary (extended length type) SCLs were different from those associated with limited length type SCLs (parallel or taper). For extended length type SCLs, there would be vehicles already occupying the SCL after entering the freeway from the previous entrance ramp terminal of the preceding interchange. Those vehicles may either continue on the SCL (i.e. the auxiliary lane) and exit the freeway, or merge left onto the freeway through lanes. Moreover, other vehicles would diverge from the freeway right most through lane onto the extended SCL for exiting the freeway. Alternatively, for limited length type SCLs, all vehicles exiting the 
freeway are those diverging from the freeway right most through lane onto the limited SCL. Whereas, the vehicles movement on the freeway adjacent right lanes would perform one of the following driving actions: merge left onto the inner lane, diverge onto the SCL (extended or limited), or continue through along the freeway right lane. Exit ramp terminals with two-lane off-ramps provided an additional alternative for vehicles travelling on the freeway right most through lane and willing to directly exit the freeway using the left-hand ramp lane. Figure 3.3 and Figure 3.4 show two field photos of an extended length type SCL and a limited length type SCL, respectively.

On extended length type SCLs (Figure 3.3), vehicles already occupying the SCL and exiting the freeway were targeted from a point upstream the freeway diverge area and along the SCL until the gore area (physical nose). While vehicles already occupying the SCL that decided to merge left onto the freeway right lane, were targeted until the individual target vehicle is half-way between the SCL and the freeway right lane. Likewise, vehicles diverging from the freeway right lane onto the limited or the extended SCLs were targeted from a point where the individual target vehicle is about to completely perform a lane change manoeuvre and along the SCL until it reaches the gore area. 


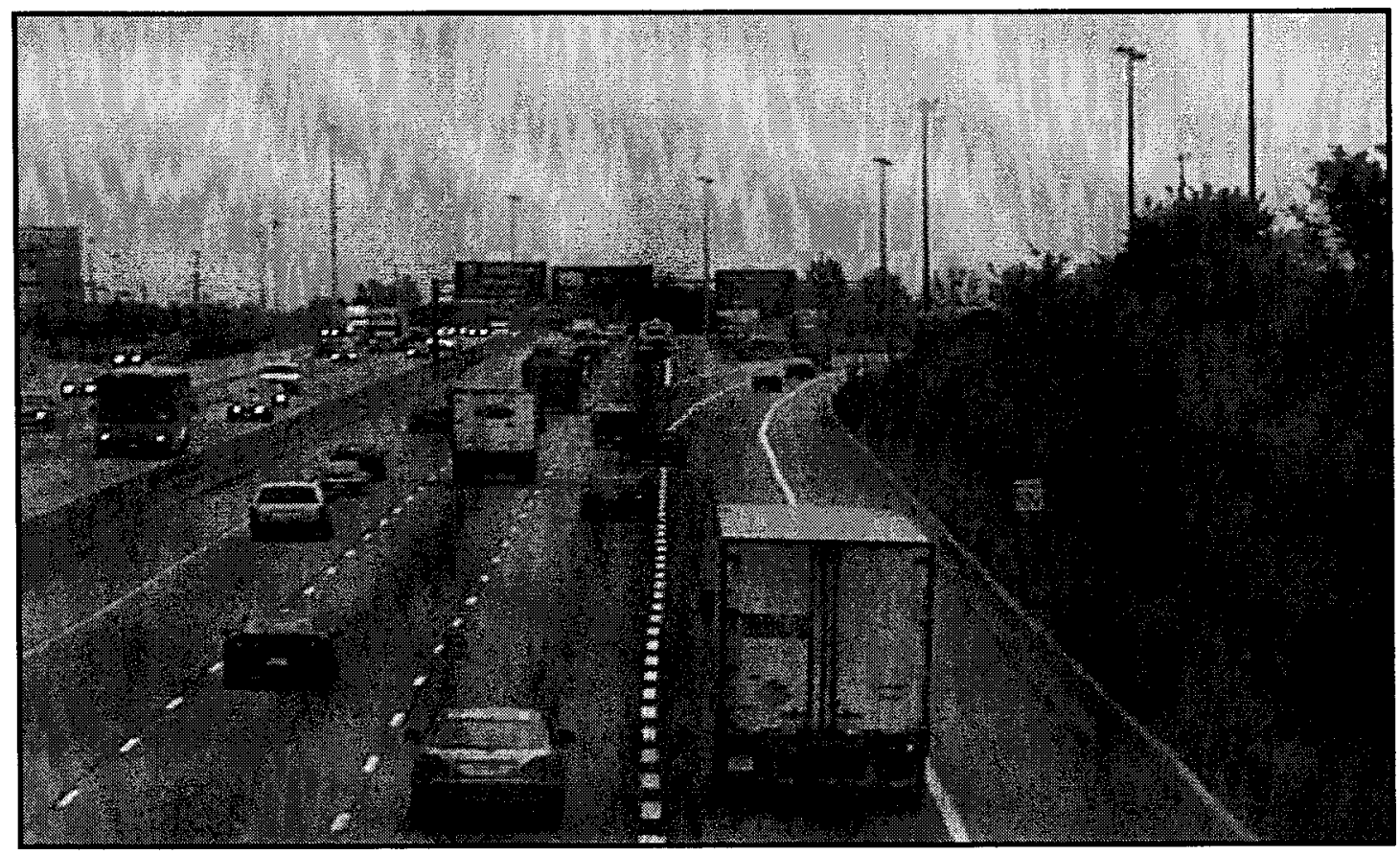

Figure 3.3: Field Photo of an Extended Length Type SCL, St. Laurent Blvd (W-NS)

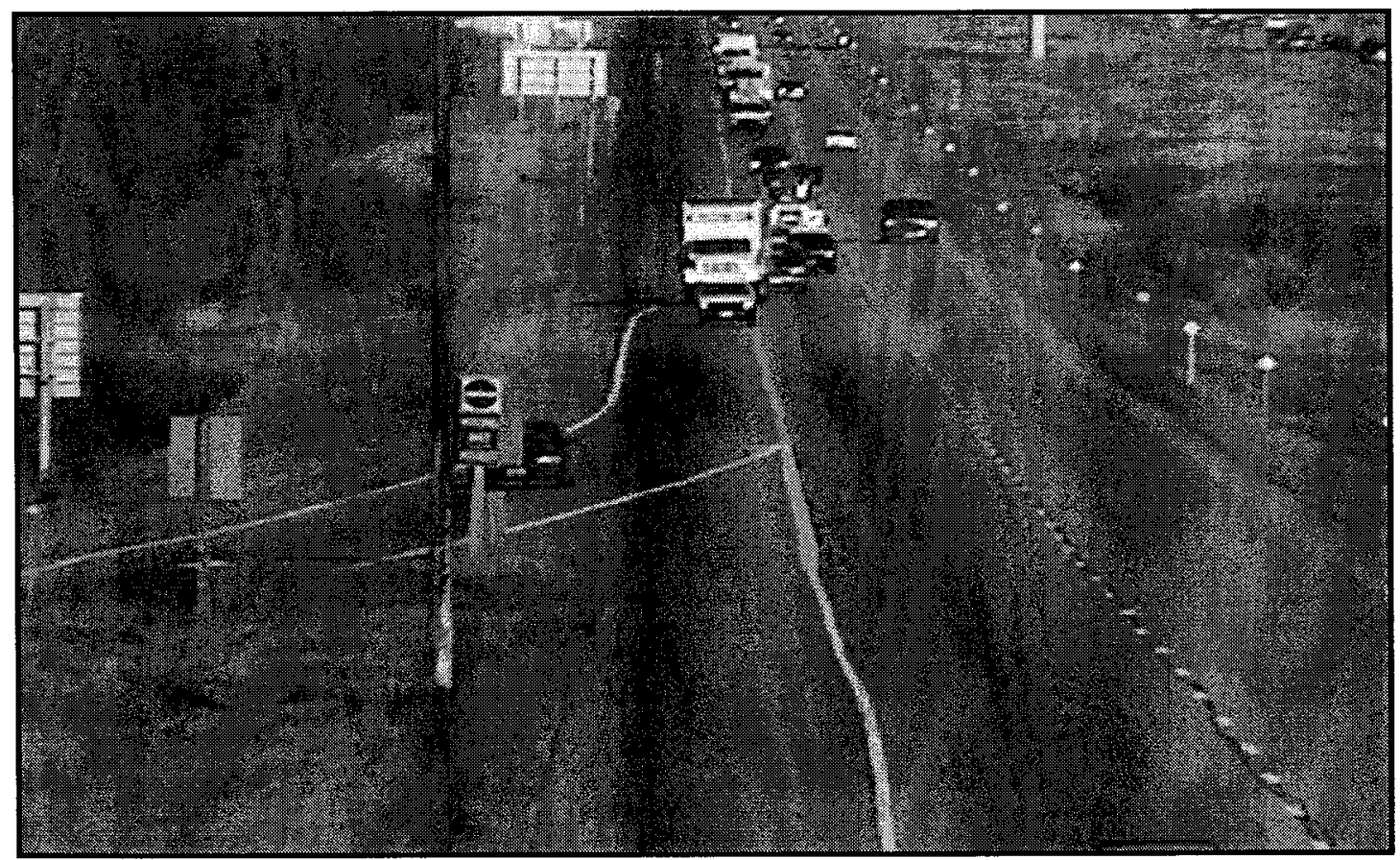

Figure 3.4: Field Photo of a Limited Length Type SCL, Carp Rd (E-NS) 
For both cases of extended and limited SCLs, vehicles on the freeway right lanes were targeted upstream the freeway diverge area and along the freeway right lane until the individual target vehicle would perform one of the following driving decisions:

- Merge left onto an inner freeway through lane,

- Diverge onto the deceleration SCL,

- Continue through on the freeway right most lane until it passes the limits of the exit ramp area, or

- Exit the freeway using the left-hand lane of the two-lane exit ramp terminals.

As previously mentioned, the targeted vehicles were manually recorded on the worksheet in the field according to their movement and vehicle classification. From Table 3.3, a corrupted file that is marked as "Out" is a speed data profile of an individual vehicle that was missed while being targeted. This could happen because another following vehicle had obstructed the targeted vehicle or because the disturbance of the SpeedLaser ${ }^{\circledR}$ equipment, due to the vibration of the overpass produced by the movement of a heavy vehicle, caused the operator to lose his target. Fortunately, not many vehicles were recorded as corrupted files during the speed data collection process, confirming the high performance of the operators in the field. Later, the corrupted files were deleted from the database right after transferring the speed data profiles to the laptop computer.

Additional work of the field scheme was to determine a typical height of a target vehicle travelling on the freeway. Therefore, the heights of a target vehicle's front and back reflective sides were measured, using a measuring tape, from the pavement surface to an approximate point where the laser light-beam of the SpeedLaser ${ }^{\circledR}$ is aimed while targeting the vehicle on the roadway. First, it was important to determine the height of the 
target vehicle approaching or receding away from the equipment position. As mentioned earlier, the travelling vehicles were targeted either from the front or from the back reflective side of the vehicle. Therefore, the front and the back average heights of three different passenger car types, in addition to four different heavy vehicle types were calculated and used for the speed correction process (only with respect to the vertical offsets). Table 3.4 shows the types of the vehicles with their front/back heights used for calculating the average vehicle heights for a target vehicle.

Table 3.4: Average Heights of Front and Back Reflective Sides for PCs \& HVs

\begin{tabular}{|c|c|c|c|}
\hline \multicolumn{4}{|c|}{ Passenger Cars (PCs) } \\
\hline & & Front & Back \\
\hline \multirow{3}{*}{ Vehicle Type } & Sedan & 0.51 & 1.00 \\
\hline & Minivan (MPV) & 0.52 & 0.97 \\
\hline & $4 \times 4$ (SUV) & 0.57 & 1.05 \\
\hline \multicolumn{2}{|c|}{ Average Target Height } & $0.53 \mathrm{~m}$ & $1.01 \mathrm{~m}$ \\
\hline \multicolumn{4}{|c|}{ Heavy Vehicles $(H V s)$} \\
\hline & & Front & Back \\
\hline \multirow{4}{*}{ Vehicle Type } & Medium Truck & 1.35 & 1.88 \\
\hline & Bus & 0.93 & 1.47 \\
\hline & Heavy Truck & 1.20 & 2.75 \\
\hline & Semi-Trailer Truck & 0.87 & 2.00 \\
\hline \multicolumn{2}{|c|}{ Average Target Height } & $1.09 \mathrm{~m}$ & $2.03 \mathrm{~m}$ \\
\hline
\end{tabular}

From Table 3.4, the typical average passenger-car front and back heights used in the speed correction procedures were 0.53 meters and 1.01 meters, respectively. On the other hand, the typical average front and back heights for a heavy vehicle were 1.09 meters and 2.03 meters, respectively. 


\subsection{SPEED DATA}

The speeds of 3,098 vehicles were recorded on the 13 freeway diverge areas selected on Highway 417 (i.e. the Queensway) in the Ottawa region to examine operational driving patterns on freeway deceleration SCLs and the adjacent right lanes. The speed data were collected during the off-peak hour periods (between 10:00 am to 3:00 pm) for a two-month period of October-to-November 2004. Subsequently, the speed data files were processed to prepare appropriate speed and deceleration driving parameters (meaningful behaviour measures) useful for modelling vehicular behaviour on freeway diverge areas, from operational and safety performance points of view. The following sub-sections cover the speed data retrieval (Section 3.3.1), the speed data processing procedures (Section 3.3.2), and finally the preparation of aggregated driver behaviour measures for the next stage of the study (Section 3.3.3).

\subsubsection{Speed Data Retrieval}

Preparation for the equipment before a field workday involved the inclusion of null files (called zero-length files) onto the PCM cards. By the end of a field workday, the speed data readings recorded on the PCM cards were retrieved to a laptop computer for later processing. For this reason, more than 350 zero-length files were created and saved on the laptop computer to be transferred to the PCM cards in the field after the files on the PCM card were utilized and retrieved. The zero-length files were created with zero capacity using the DOS language for recording speed data readings for the targeted vehicles. The recorded speed data readings are readable by the MS-Excel software, and 
Figure 3.5 shows a sample output file of the SpeedLaser ${ }^{\circledR}$ speed data readings for an individual vehicle recorded on the deceleration SCL on Bronson Ave (W-NS).

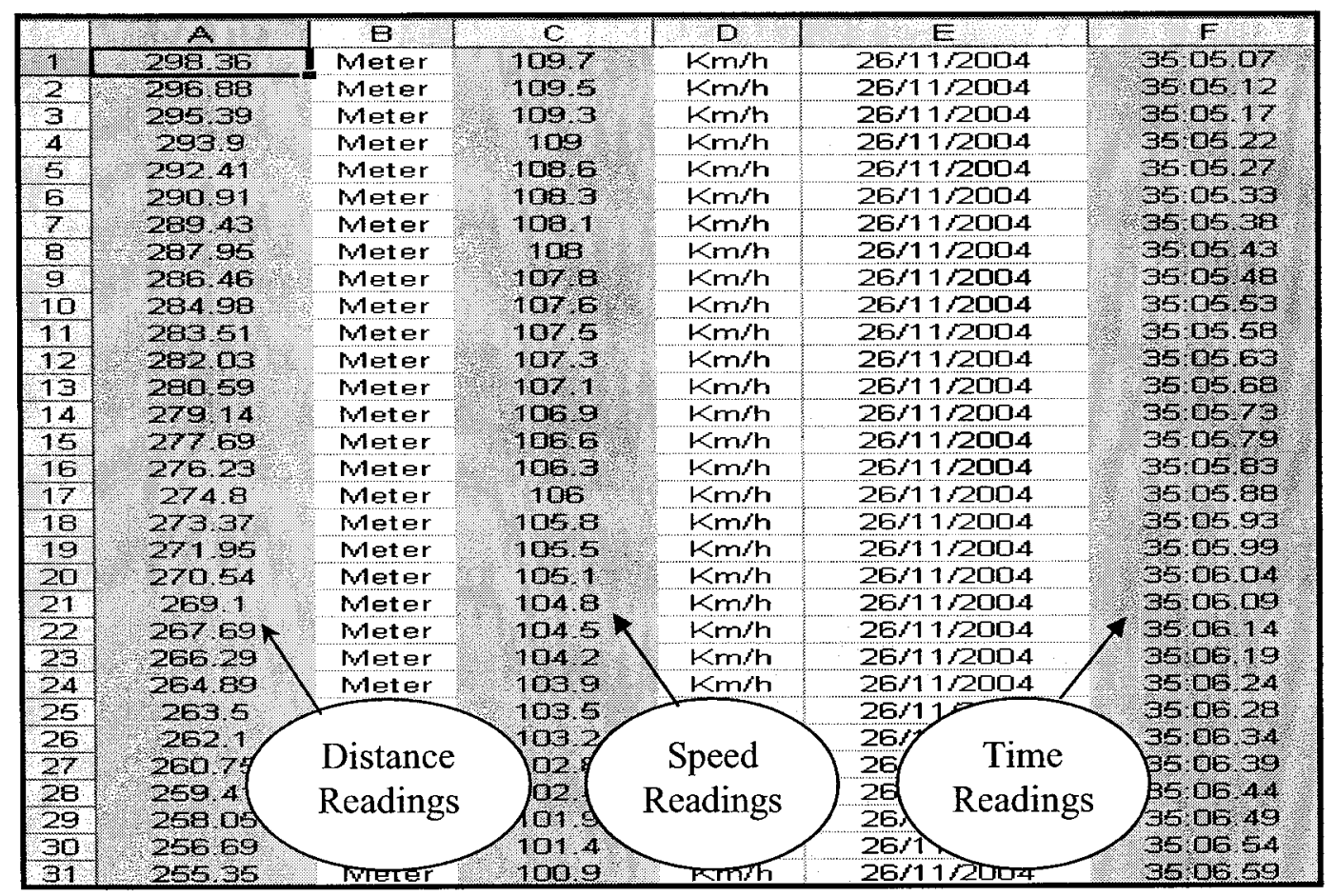

Figure 3.5: SpeedLaser ${ }^{\circledR}$ Speed Data Readings File Sample

As seen in Figure 3.5, the SpeedLaser ${ }^{\circledR}$ output files include speed, distance, and time measurements per each reading row. On each row, the distance of a vehicle's position from the equipment position is given in meters along with the relative speed value in $\mathrm{km} / \mathrm{h}$. These output data points of a vehicle represent the driver's behaviour along the freeway section travelled by the vehicle while being targeted. Hence, the recorded data were first processed and then used to extract meaningful operational parameters (driving behaviour measures) that help model the drivers' behaviour on freeway diverge areas (i.e. deceleration SCLs and the adjacent right lanes). 


\subsubsection{Speed Data Processing}

First, a visual review for all speed data profiles (excel files), collected from the thirteen SCLs and the adjacent right lanes, was carried out to detect and delete the data points (rows) that have no readings or have repeated distance and time readings. Generally, the data points that have no readings resulted from the equipment's trigger release by fault or the loss of the targeted vehicle and then retargeting it. While, the repeated distance and time readings result from the equipment's precisions for speed, distance, and time measurements. The SpeedLaser ${ }^{\circledR}$ precision of $0.1 \mathrm{~km} / \mathrm{h}, 0.01$ meters and 0.01 seconds for speed, distance, and time, respectively, would sometimes allow the speed readings to be recorded with no change in the distance or time readings.

Further visual reviews regarding the identification of the data points that do not integrate within an individual speed profile, due to the targeting of another vehicle on the roadway, were successfully completed for all the data profiles collected for the purpose of this study. Afterwards, the conversion of negative speed data readings recorded for vehicles receding away from the operator's position were also performed successfully. Finally, the 3,098 speed profiles collected for the purpose of this study $(1,550$ on freeway SCLs and 1,548 on the adjacent right lanes) were grouped based on the vehicle classification (passenger cars and heavy vehicles), and their movement on the freeway. This was executed by utilizing the relevant field worksheets that were used to record vehicle class and movement on the freeway diverge areas (refer to Table 3.3).

Afterwards, all the speed data profiles were used for correction calculations with respect to the vertical and horizontal offsets that were measured on the field for the relevant selected sites. Next is a detailed description of the methodology carried out for 
the speed correction procedures for all the speed data profiles collected on the freeway SCLs and the adjacent right lanes.

\subsection{2.a Correction for Vertical and Horizontal Offsets}

As mentioned earlier, the heights of the equipment from the freeway's pavement surface to the mid-point between the two lenses of the SpeedLaser ${ }^{\circledR}$, measured at each of the respective sites selected for this study, were determined for the purpose of the vertical and horizontal offset corrections. Again, the vertical offsets $(Y)$ were calculated as the height of the equipment $\left(H_{E q}\right)$ (measured from the freeway pavement surface) minus the typical average height of the target vehicle $\left(H_{V e h}\right)$ (presented in Table 3.4). On the other hand, in case of an equipment position not located on the extension of the centerline of the respective lane of the targeted vehicle, the horizontal offsets $(X)$ were measured on the overpass's sidewalk surface from the equipment position to the extension of the centerline of the occupied lane. The vertical and horizontal offsets for all sites ranged from 7.26 meters to 12.59 meters and from 0.0 meters to 5.2 meters, respectively.

Depending on the equipment position to the respective lane occupied by a targeted vehicle on the freeway, there were two possible positions to be considered when correcting for speed data profiles. First, when the equipment was located on the extension of the centerline of the occupied respective lane, the speed profile of a targeted vehicle is to be corrected for the vertical offset only. While, the other possible position to be considered was when the equipment was shifted away from the extension of the centerline of the occupied respective lane, and so the speed profile of a targeted vehicle is to be corrected for both the vertical and horizontal offsets. Figure 3.6 and Figure 3.7 
graphically show the two possible equipment positions while targeting a travelling vehicle on the freeway lane (SCL or through right lane).

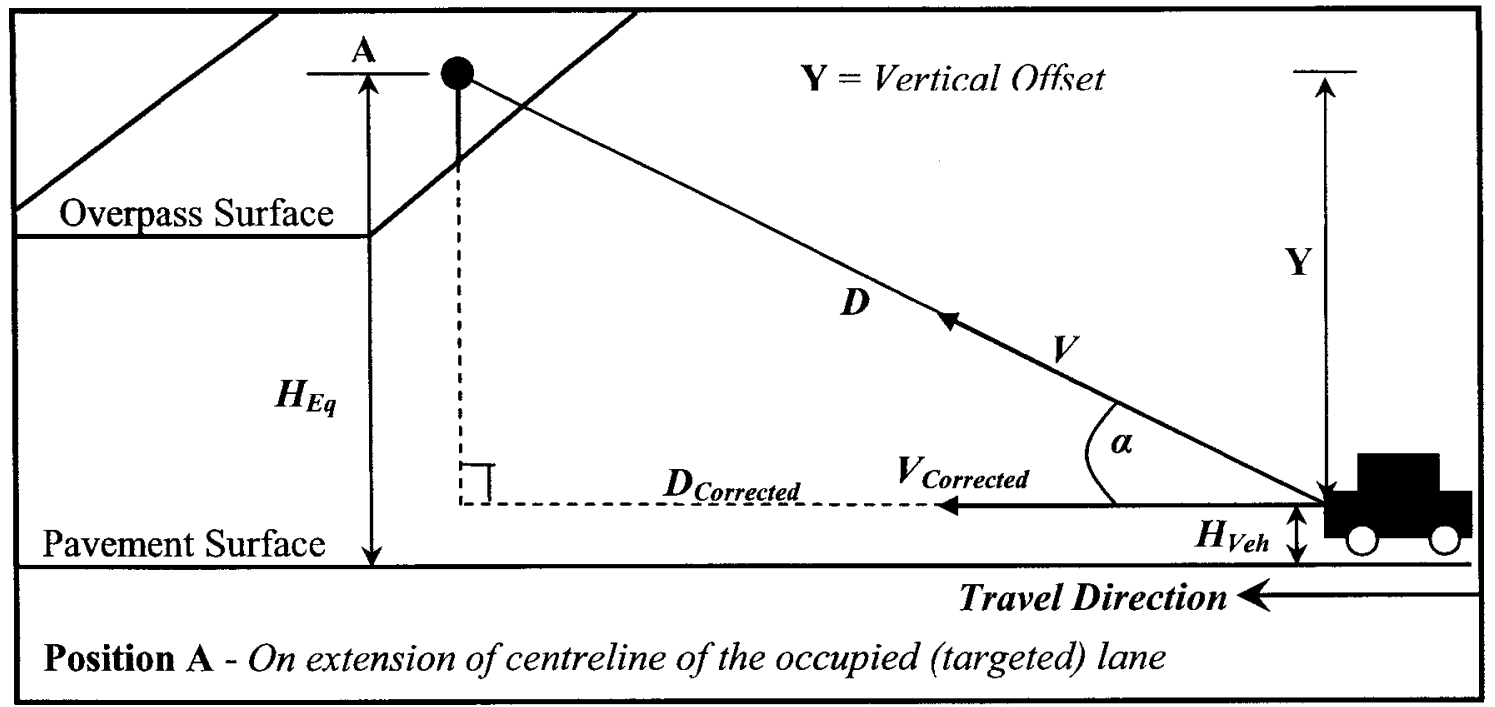

Figure 3.6: Vertical Offset Correction

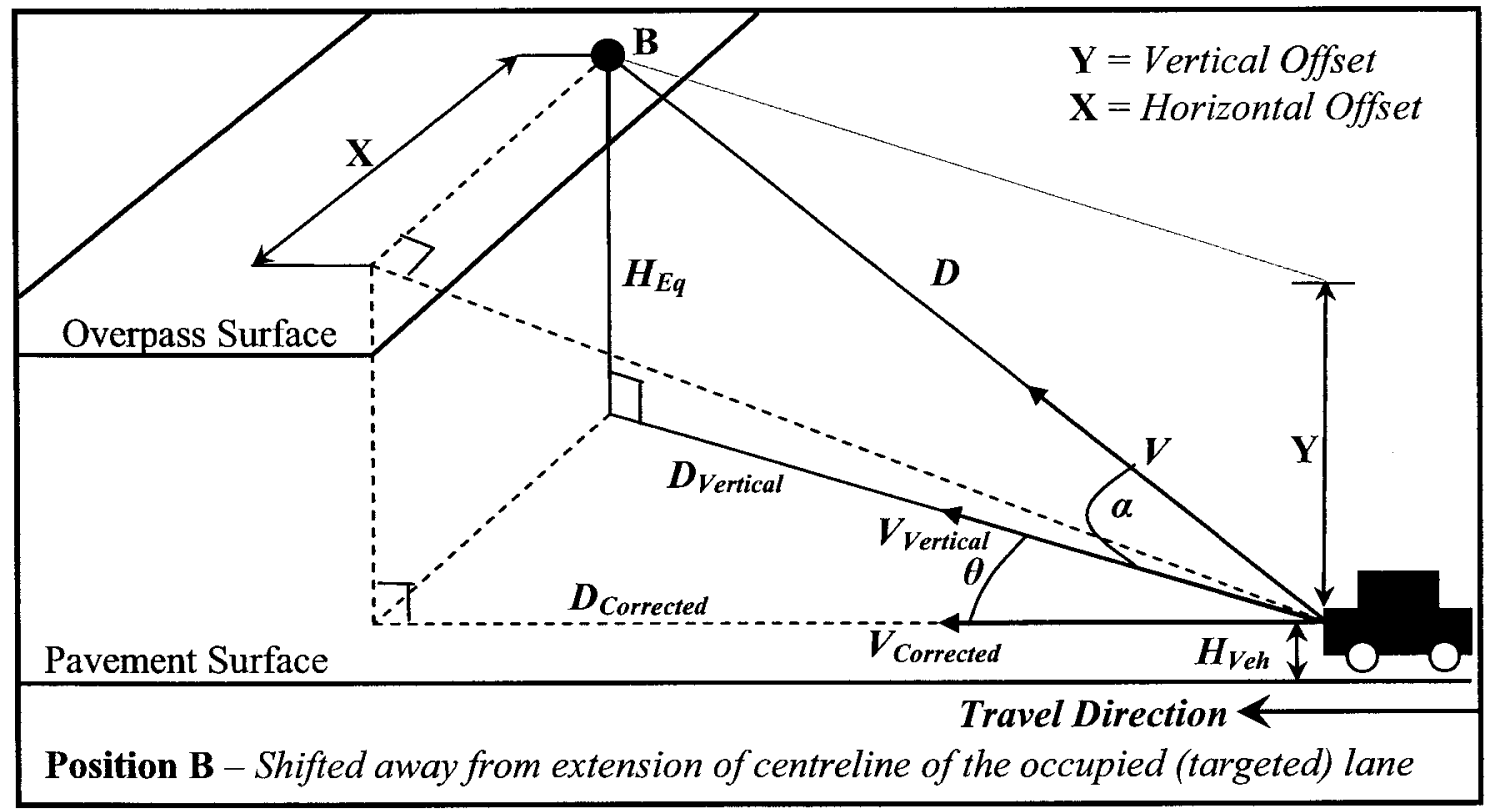

Figure 3.7: Vertical and Horizontal Offset Corrections 
Figure 3.6 shows a sample equipment position (Position ' $\mathrm{A}$ ' on the figure) that is located on the extension of the centerline of the occupied respective lane while targeting a travelling vehicle on the freeway lane (SCL or through right lane). On the other hand, Figure 3.7 shows a sample equipment position (Position ' $\mathrm{B}$ ' on the figure) shifted away from the extension of the centerline of the occupied respective lane while targeting a travelling vehicle on the freeway SCL lane or through right lane. The 3-D illustrations shown on the figures facilitate the correction for vertical offset only or both the vertical and horizontal offset, where applicable. The Pythagoras theorem and vector mechanics principles together were implemented for the speed and distance correction tasks. For any of the two equipment positions presented in Figure 3.6 and Figure 3.7, the right-angled triangle created in the vertical plane between the targeted vehicle and the equipment position has its three sides as follows:

- The height as the vertical offset $(Y)$.

- The hypotenuse as the measured distance $(D)$ with the recorded speed $(V)$ vector.

- The base as the corrected distance $\left(D_{\text {Corrected }}\right)$ with the corrected speed $\left(V_{\text {Corrected }}\right)$ vector.

Given the hypotenuse and the height values of the vertical right-angled triangle, the base corrected distance and the vertical offset corrected speed were calculated using the following trigonometry ratios:

$$
\begin{aligned}
& D_{\text {Corrected }}=\sqrt{D^{2}-Y^{2}} \\
& \operatorname{Cos} \alpha=D_{\text {Corrected }} / D
\end{aligned}
$$




$$
V_{\text {Corrected }}=V / \operatorname{Cos} \alpha=V \times\left(D / D_{\text {Corrected }}\right)
$$

Additionally, the equipment position that have created vertical and horizontal right-angled triangles as presented in Figure 3.7 and would therefore require corrections for both vertical and horizontal offsets. Again, the right-angled triangle created in the vertical plane between the targeted vehicle and the equipment position has its three sides as follows:

- The height as the vertical offset $(Y)$.

- The hypotenuse as the measured distance $(D)$ with the recorded speed $(V)$ vector.

- The base as the vertical corrected distance $\left(D_{\text {Vertical }}\right)$ with the vertical corrected speed $\left(V_{\text {Vertical }}\right)$ vector.

Then, the right-angled triangle created in the horizontal plane between the targeted vehicle and the equipment position has its three sides as follows:

- The height as the horizontal offset $(X)$.

- The hypotenuse as the vertical corrected distance $\left(D_{\text {Vertical }}\right)$ with the vertical corrected speed $\left(V_{\text {Vertical }}\right)$ vector.

- The base as the corrected distance ( $\left.D_{\text {Corrected }}\right)$ with the corrected speed $\left(V_{\text {Corrected }}\right)$ vector.

The correction process for both the vertical in addition to the horizontal offset correction follows the same concept as the correction for the vertical offset only through the implementation of Equation 3.1 as well as using the following equations:

$$
D_{\text {Corrected }}=\sqrt{D_{\text {Vertical }}^{2}-X^{2}}
$$




$$
\begin{gathered}
\operatorname{Cos} \theta=D_{\text {Corrected }} / D \\
V_{\text {Corrected }}=V / \operatorname{Cos} \theta=V \times\left(D / D_{\text {Corrected }}\right)
\end{gathered}
$$

\subsection{2.b Corrected Distance and Speed Calculations}

In case of a vehicle targeted from an equipment position that is exactly on the centerline of the occupied respective freeway lane, Equations 3.1 and 3.3 are the only two equations to be used for calculating the corrected distances and speeds. It should be noted that the calculations performed took into consideration the direction of travel of the target vehicle (approaching or receding) with the vehicle classification (PC or HV), where applicable. The direction of travel of a targeted vehicle was considered in determining the typical height of the vehicle's front or back side according to its class to calculate the vertical offset measurements. The vertical angles $(\alpha)$ and the horizontal angles $(\theta)$ that were created with relevance to the vehicle's position on the freeway from the equipment position were less than $5^{\circ}$ and have resulted in very small correction measurements for

the distance and the speed values collected using the SpeedLaser ${ }^{\circledR}$. Specifically, the vertical and the horizontal offset corrections increased the vehicle speeds by $0.1 \mathrm{~km} / \mathrm{h}$ up to $1.4 \mathrm{~km} / \mathrm{h}$ and slightly decreased the vehicle distances by 0.02 meters up to 1.09 meters.

\subsection{2.c Deceleration Calculations and Adjustment of Speed Profiles}

As mentioned earlier, among the objectives of this study is to evaluate the drivers' deceleration behaviour on the freeway deceleration SCLs. Therefore, the deceleration rates between each two successive data points (rows) for all vehicles on the freeway deceleration SCLs were calculated through the application of equations of motion. The 
distance and the time readings recorded by the SpeedLaser ${ }^{\circledR}$ were used to determine distance and time intervals between each two successive data points. This was then followed by the calculation of the deceleration rates based on these distance and time intervals.

The distance and time intervals, determined along an individual vehicle profile, were both incorporated into the calculations of the deceleration rates by means of two distinctive equations of motion namely; the velocity-time and velocity-displacement relationships. These two equations are symbolized as follows:

$$
\begin{aligned}
& d_{\text {Time }}=\frac{V_{f}-V_{o}}{T_{\text {Interval }}} \\
& d_{\text {Dist }}=\frac{V_{f}^{2}-V_{o}^{2}}{2 \times D_{\text {Interval }}}
\end{aligned}
$$

Where, $d_{\text {Time }}=$ the deceleration rate between the two successive data points based on the time interval $\left(\mathrm{m} / \mathrm{sec}^{2}\right) ; d_{D i s t}=$ the deceleration rate between the two successive data points based on the distance interval $\left(\mathrm{m} / \mathrm{sec}^{2}\right) ; V_{f}=$ the final speed at the second data point $(\mathrm{m} / \mathrm{sec}) ; V_{o}=$ the initial speed at the first data point $(\mathrm{m} / \mathrm{sec}) ; T_{\text {Interval }}=$ the travel time interval between the two successive data points $(\mathrm{sec}) ; D_{\text {Interval }}=$ the distance interval between the two successive data points $(\mathrm{m})$

Certainly, for each interval of a speed profile, the two successive speed data readings bounding the interval represent the initial speed $\left(V_{o}\right)$ and the final speed $\left(V_{f}\right)$. The calculated deceleration/acceleration rates along an individual speed data file resulted in values with negative or positive signs indicating a deceleration or an acceleration rate, respectively. It was noted that the driver/vehicle behaviour on the freeway deceleration 
SCLs incorporated partial acceleration behaviour, depending on the geometry of the SCL, the traffic volumes, the diverging speed from the freeway right most through lane onto the deceleration SCL, and/or the gore speed at the freeway exit gore area.

The calculations of the deceleration/acceleration rates based on time and distance intervals ( $d_{\text {Time }}$ and $d_{D i s t}$ ) for all the speed data files that were collected on the freeway deceleration SCLs were performed successfully. Afterwards, the successive deceleration/acceleration rates, calculated using Equations 3.7 and 3.8, were compared to sometimes find deceleration rates that were calculated based on the distance interval have higher values than those calculated based on the time taken to travel such distance interval. The reason for such impractical situations inherent in the fact that there were few distance intervals with very small values (less than 0.5 meters) and therefore have generated very high deceleration/acceleration rates. Consequently, adjustments for all speed profiles on the freeway deceleration SCLs were carried out to maintain an interval value between each two successive speed data point readings capable of capturing changes in vehicle's manoeuvres on the freeway deceleration SCL. These adjustments were accomplished by deleting an intermediate speed data point reading bounding the deceleration/acceleration rates under question (i.e. a very high deceleration/acceleration rates calculated based on distance). The criterion used in examining the SCL speed profiles was that the two deceleration/acceleration rates calculated using both the velocity-time and velocity-displacement relationships should be of almost the same value. Afterwards, for each SCL speed profile, the average $\left(d_{\text {Average }}\right)$ of the two deceleration/acceleration rates was calculated as the final rate for analysis purposes in this study. 
As previously mentioned, with regard to the freeway deceleration SCL speed profiles, vehicles were targeted as they diverge onto the deceleration SCL and while they travel along the SCL until a point nearby the gore area (physical nose). After a targeted vehicle travel on the deceleration SCL and reaches the gore area, the operator decides to stop shooting his target somewhere at the proximity of the gore area. Thus, the speed profiles collected on the freeway deceleration SCLs had different extents. Afterwards, the determination of an absolute reference point (for example the beginning of the controlling ramp-curve or the end of the freeway deceleration SCL) to which all the SCL speed profiles should be adjusted to that same point was necessary for consistency of all the SCL speed profiles. As a result, the length of the freeway deceleration SCL (see Section 3.4.2.a for SCL measurements) was defined to be the effective area for evaluating the driver behaviour as confined by the study objectives.

Suggestions involved interpolation and/or extrapolation calculations to adjust for longer and/or shorter speed profiles with respect to the end of the relevant deceleration SCL. Longer speed profiles involved interpolation calculations to set for the point at which the deceleration SCL ends, with respect to the equipment position. The distances from the equipment position to the end of the selected deceleration SCLs were determined using the Engineering and Title Records (ETR) geometric plans, provided by the Ontario Ministry of Transportation (MTO). Having these distances, interpolations were performed between the two successive distance readings at which the relevant deceleration SCL ends to calculate corresponding speed and time readings of a vehicle profile at that certain location. Afterwards, the rest of an interpolated speed profile was erased (i.e. speed data recorded from the end of the deceleration SCL to the proximity of 
the gore area). On the other hand, concerning short speed profiles, where a targeted vehicle has the last distance reading shorter than the distance measured from the equipment position to the end of the SCL, decisions were taken not to implement extrapolation calculations to prevent any misleading results. In the end, all the SCL speed profiles were adjusted to the end of the corresponding deceleration SCL with the exclusion of short speed profiles.

\subsubsection{Preparation of Aggregated Driver Behaviour Measures}

All the speed profiles, collected on freeway deceleration SCLs and the adjacent right lanes on Highway 417, were used to determine driving behaviour parameters for the analysis procedures for the purpose of this study. Decisions were taken to determine and calculate meaningful and significant parameters competent in representing the speed profiles collected on the freeway deceleration SCLs and the adjacent right most through lanes. The following parameters listed below are those calculated for each vehicle collected on the freeway right most lanes and that to be used in the analysis of this study.

- Mean Speed - for vehicles travelled on the freeway right most lane until they pass the limits of the exit ramp area.

- Diverge Speed - for vehicles travelled on the freeway right most lane and then diverged onto the SCL.

Figure 3.8 shows a sample of a typical right lane corrected speed profile with the calculated driving behaviour measures that represent the relevant targeted vehicle. The typical corrected speed profile on the freeway right lane, graphically illustrated in Figure 3.8 , simply describes the overall behaviour of the targeted vehicle on a freeway right 
lane. As mentioned earlier, the distance readings (corrected for vertical and horizontal offsets) refer to the vehicle's position as it travel on the freeway roadway with respect to the SpeedLaser ${ }^{\circledR}$ position. In Figure 3.8, the mean speed for an individual vehicle was calculated as the average of all the successive corrected speed readings, while the diverge speed was determined as the last corrected speed reading of the targeted vehicle that diverged onto the deceleration lane.

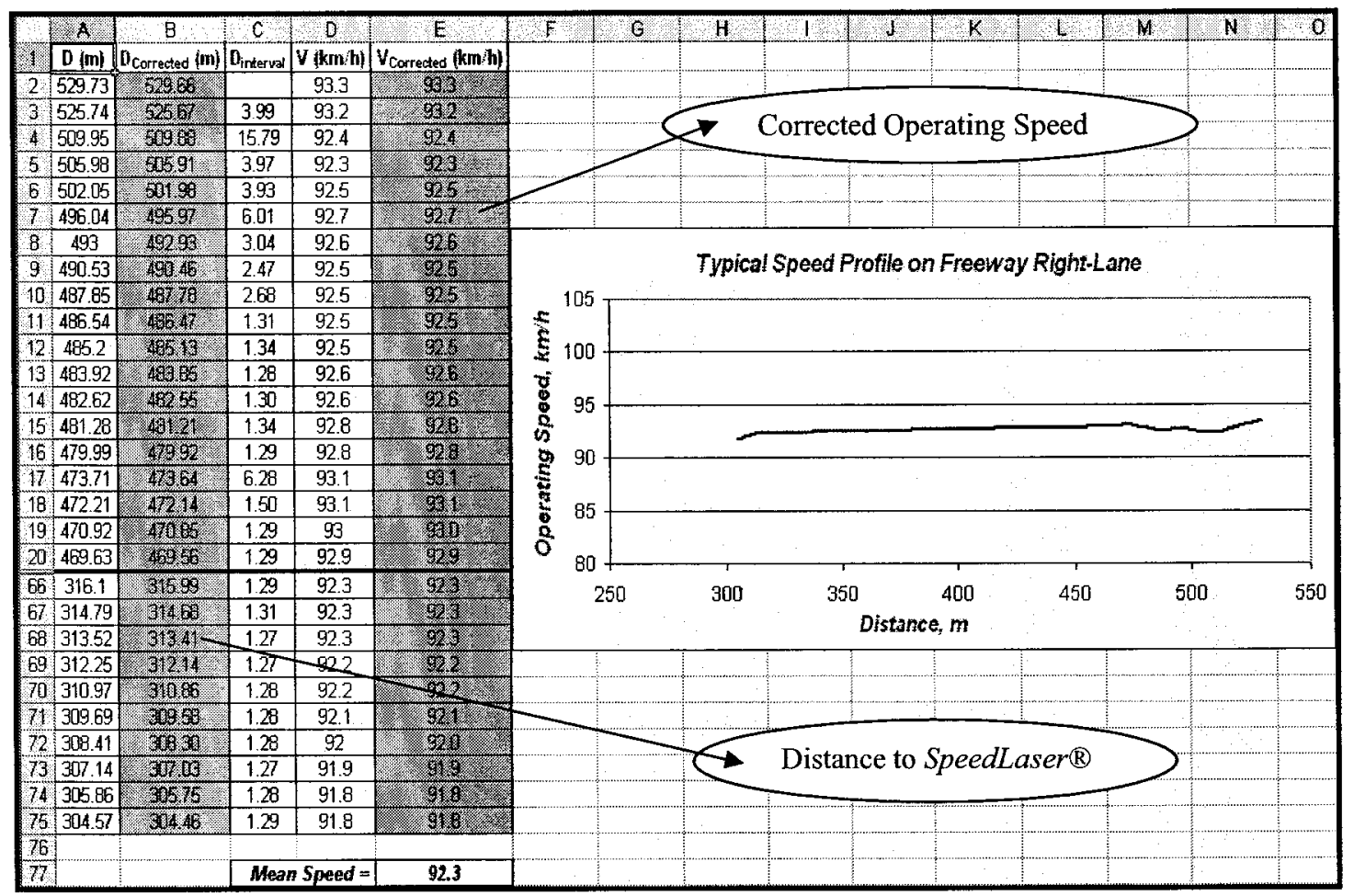

Figure 3.8: Right-Lane Corrected Speed Profile Sample

On the other hand and after finishing all work related to the freeway right-lane speed profiles, it was necessary to process the speed profiles collected on freeway deceleration speed-change lanes. The following parameters are those determined and calculated for each vehicle (speed profile) diverged from the main traffic and travelled on 
the freeway deceleration SCL before exiting the freeway, for use in the driver behaviour analysis of this study.

- Diverge Speed

- Maximum Speed

- Mean Speed

- Gore Speed

- Maximum Deceleration Rate

- Mean Deceleration Rate

- Overall Deceleration Rate

- Deceleration Distance travelled from diverge point to end of the SCL

Figure 3.9 shows a sample of a corrected speed profile for a targeted vehicle on a freeway deceleration SCL with the calculated necessary driving behaviour parameters. The corrected speed profiles for all the targeted vehicles on the freeway deceleration SCLs were processed and compiled into groups (according to the vehicle class and their movements on the freeway) to express the overall behaviour on the freeway diverge areas. 


\begin{tabular}{|c|c|c|c|c|c|c|c|c|c|c|c|c|c|c|}
\hline & X) & $\mathrm{B}$ & $c$ & 0 & E & $F$ & $G$ & $H$ & 1 & d & K & L & $M$ & $N$ \\
\hline 1. & $0(m)$ & $D_{\text {Verticat }}(\mathrm{m})$ & D $_{\text {Horizontal }}(\mathbf{m})$ & $D_{\text {Irtervad }}$ & Cum. D D nterval & $V(\mathrm{~km} / \mathrm{h})$ & $V_{\text {Latreceof }}(\operatorname{math})$ & $\mathrm{v}_{\text {Corrected }}(\mathrm{m} / \mathrm{sec})$ & Time & $\mathrm{T}_{\text {indervat }}$ (sec) & Cum. $1_{\text {Irierual }}$ & $d_{\text {time }}\left(\mathrm{m} / \mathrm{s}^{2}\right)$ & $d_{\text {gistance }}\left(m / s^{2}\right)$ & $\mathrm{d}_{\text {puerage }}\left(\mathrm{m} / \mathrm{s}^{2}\right)$ \\
\hline 2 & 595.01 & 594.96 & 594.94 & & & 97.6 & 96 & 27.1 & 55.34 .73 & & 0 & & & 5 \\
\hline 3. & 593.59 & 59354 & 593.52 & 1.42 & 1.42 & 970 & 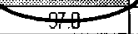 & 27.2 & 55.34 .79 & 0.06 & 0.06 & 0.926 & 1.062 & 1964 \\
\hline 4 & 592.38 & 592.33 & \multirow{3}{*}{\multicolumn{3}{|c|}{ Diverge Speed }} & 98 & 98.0 & 27.2 & \begin{tabular}{|l|l|}
$55: 34.34$ \\
\end{tabular} & 0.05 & 0.11 & 1.112 & 1.249 & $1 / 160$ \\
\hline 5 & 590.74 & 590.69 & & & & 98.3 & 98.3 & 27.3 & $55: 34.89$ & 0.05 & 0.16 & 1.667 & 1.386 & $1 \times 1 x^{2}$ \\
\hline 6 & 589.3 & 589.25 & & & & 98.7 & 98.7 & 27.4 & \multirow{2}{*}{\multicolumn{4}{|c|}{ Deceleration/Acceleration }} & 2.112 & Th \\
\hline 7 & 587.9 & 587.65 & 587.83 & 1.40 & 7.11 & 99 & 99.0 & 27.5 & & & & & 1.635 & 18Y) \\
\hline 8 & 586.51 & 586.46 & 586.44 & 1.39 & 8.50 & 99.2 & 99.2 & 27.6 & & & & & 1.101 & (10) \\
\hline 9 & 585.1 & 58505 & 585.03 & 1.41 & 9.91 & 99.3 & 99.3 & 27.6 & & & & & 0.544 & 1360 \\
\hline 10 & 583.79 & 50374 & 58.72 & 1.31 & 11,22 & 99.4 & 99.4 & 27.6 & $55: 35.14$ & 0.05 & 0.41 & 0.556 & 0.586 & 031 \\
\hline 11 & 582.54 & 582.49 & 582.47 & 1.25 & 12.47 & 99.4 & 99.4 & 27.6 & $55: 35.19$ & 0.05 & 0.46 & 0.100 & 0.000 & 1) \\
\hline 151 & 294.51 & 294.41 & 294.36 & 1.07 & 300.57 & 77.3 & 77.3 & 21.5 & $55: 46.57$ & 0.05 & 11.84 & -0.554 & 2557 & 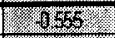 \\
\hline 152 & 293.42 & 29332 & 293.27 & 1.09 & 301.66 & 77.1 & 77.1 & 21.4 & \begin{tabular}{|l|}
55.45 .62 \\
\end{tabular} & 0.05 & 11.89 & -1.110 & -1.098 & III) \\
\hline 153 & 292.33 & 292.23 & 292.18 & 109 & 302.76 & 77.1 & 77.1 & 21.4 & $55: 46.67$ & 0.05 & 11.94 & 0.002 & 0.002 & $0 \mathrm{WH}$ \\
\hline 154 & 291.23 & 291.13 & 291.08 & 1.10 & 303.86 & 77.1 & 77.1 & 21.4 & 55.46 .72 & 0.05 & 11.99 & 0.002 & 0.002 & 1010 \\
\hline 166 & 290.13 & 290.03 & 289.98 & 110 & 304.96 & 77.2 & \multirow{3}{*}{\multicolumn{2}{|c|}{ Gore Speed }} & $55: 46.77$ & 0.05 & 12.04 & 0.557 & 0.543 & 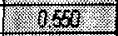 \\
\hline 156 & 289.03 & 208.93 & 286.86 & 1.10 & 306.06 & 77.3 & & & $55: 46.82$ & 0.05 & \multirow{3}{*}{\multicolumn{3}{|c|}{ Overall Time }} & (1) \\
\hline 15 & $287.97^{2}$ & \multirow{3}{*}{\multicolumn{3}{|c|}{ Overall Distance }} & 307.16 & 775 & & & 55.46 .87 & 0.05 & & & & y. \\
\hline 158 & 2868 & & & & 308.24 & 77.6 & 776 & 21.6 & $55: 46.92$ & 0.05 & & & & 1.67\% \\
\hline 159 & 285. & & & & 309.29 & 77.6 & 67.6 & 21.6 & 55:46.98 & 0.06 & 127 & 0.001 & 0.002 & MOV \\
\hline 160 & 284.74 & 284.64 & 284.59 & 1.05 & 310.35 & 77.6 & 77.6 & 21.6 & 56.47 .03 & 0.05 & 3 & 0.002 & 0.002 & Ora \\
\hline 61 & 283.69 & 283.59 & 283.54 & 1.05 & गा1.40 & 77.5 & 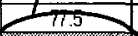 & 21.5 & $55: 47.09$ & 0.05 & & -0.554 & -0.568 & W161 \\
\hline 162 & 282.66 & 282.56 & 262.51 & $t .01$ & XII 3 & 77.2 & 72 & 21.5 & $55: 47.13$ & 0.05 & 14 & -1.666 & -1.738 & 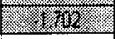 \\
\hline \multicolumn{15}{|l|}{63} \\
\hline 164 & & & & & & & & & & & & Maximum D & ecceleration $=$ & -2.065 \\
\hline 185 & & & & \multicolumn{3}{|c|}{$<$ Overall Deceleration time $=$} & -0.456 & & \multicolumn{3}{|c|}{ Average of } & \multicolumn{2}{|c|}{ Mean Decelesation = } & -0.440 \\
\hline 66 & & & & \multicolumn{3}{|c|}{ Overall Deceleration_Distance $=$} & -0.440 & & & & & \multicolumn{2}{|c|}{ Overall Deceleration $=$} & -0.448 \\
\hline 167 & & & & & & & & & & & & \multicolumn{2}{|c|}{ Diverge Speed $=$} & 97.6 \\
\hline 160 & & & & & & & & & & & & \multicolumn{2}{|c|}{ Maximtm Speed $=1$} & 99.5 \\
\hline 169 & & & $\mathrm{Eq}$ & tip & 7 & & & & & & & \multicolumn{2}{|c|}{ Mean Speed $=$} & 90.7 \\
\hline 179 & & & & & & & & & & & & \multicolumn{2}{|r|}{ Gore Speed = } & 77.2 \\
\hline 171 & & & & & & & & & & & & \multicolumn{2}{|c|}{ Deceleration Distance $=$} & 312.43 \\
\hline
\end{tabular}

Figure 3.9: Speed-Change Lane Corrected Speed Profile Sample

From the sample, illustrated in Figure 3.9, the diverge speed was determined as the first corrected speed data reading of the targeted vehicle (speed profile). On the other hand, the last speed data reading of a corrected speed profile represents the gore speed, which was recorded at the proximity of the gore area. The maximum speed reached by a targeted vehicle on the deceleration SCL was determined as that maximum speed data point of all the successive corrected speed readings along the speed profile. Finally, the mean speed was calculated as the average of all the successive speed data readings of the corrected speed profile. Moreover, as shown in Figure 3.9, the maximum deceleration rate along a vehicle's profile on a deceleration SCL was calculated as the minimum deceleration value of all the successive deceleration/acceleration rates, on account of the negative sign of the deceleration rates. The mean deceleration rate of a vehicle's profile 
along a freeway deceleration SCL was calculated as the average of all the successive deceleration/acceleration rates. Moreover, the overall deceleration rate was computed as the average of the two individual overall deceleration rates, calculated based on the overall distance travelled on the freeway deceleration SCL and the overall time elapsed during such deceleration behaviour. It should be noted that the overall deceleration rate considers the entire vehicle's profile as one interval. Finally, the effective deceleration distance used for deceleration on the freeway SCL is another important parameter and was calculated as the total distance travelled on the deceleration lane from the diverge point to the end of the deceleration SCL.

Afterwards, all of the diverge speeds, the gore speeds, the maximum speeds, the mean speeds, the overall deceleration rates, the maximum deceleration rates, the mean deceleration rates, and the effective deceleration distances were summarized based on vehicle classification (PC or $\mathrm{HV}$ ) and vehicle movement. Figure 3.10 and Figure 3.11 show two summarized samples of all the analysis parameters (driving behaviour measures) calculated for all vehicles targeted on a limited length type deceleration SCL (Carp E-NS) and a dual-exit extended length type auxiliary SCL (Greenbank E-NS), respectively. Since short speed profiles have their last speed readings not representing the gore speed, therefore decisions were taken to mark short speed profiles as "short movement" and not to consider any of their driving behaviour parameters for further analysis, except for maximum deceleration rate, diverge speed, and the maximum speed. 


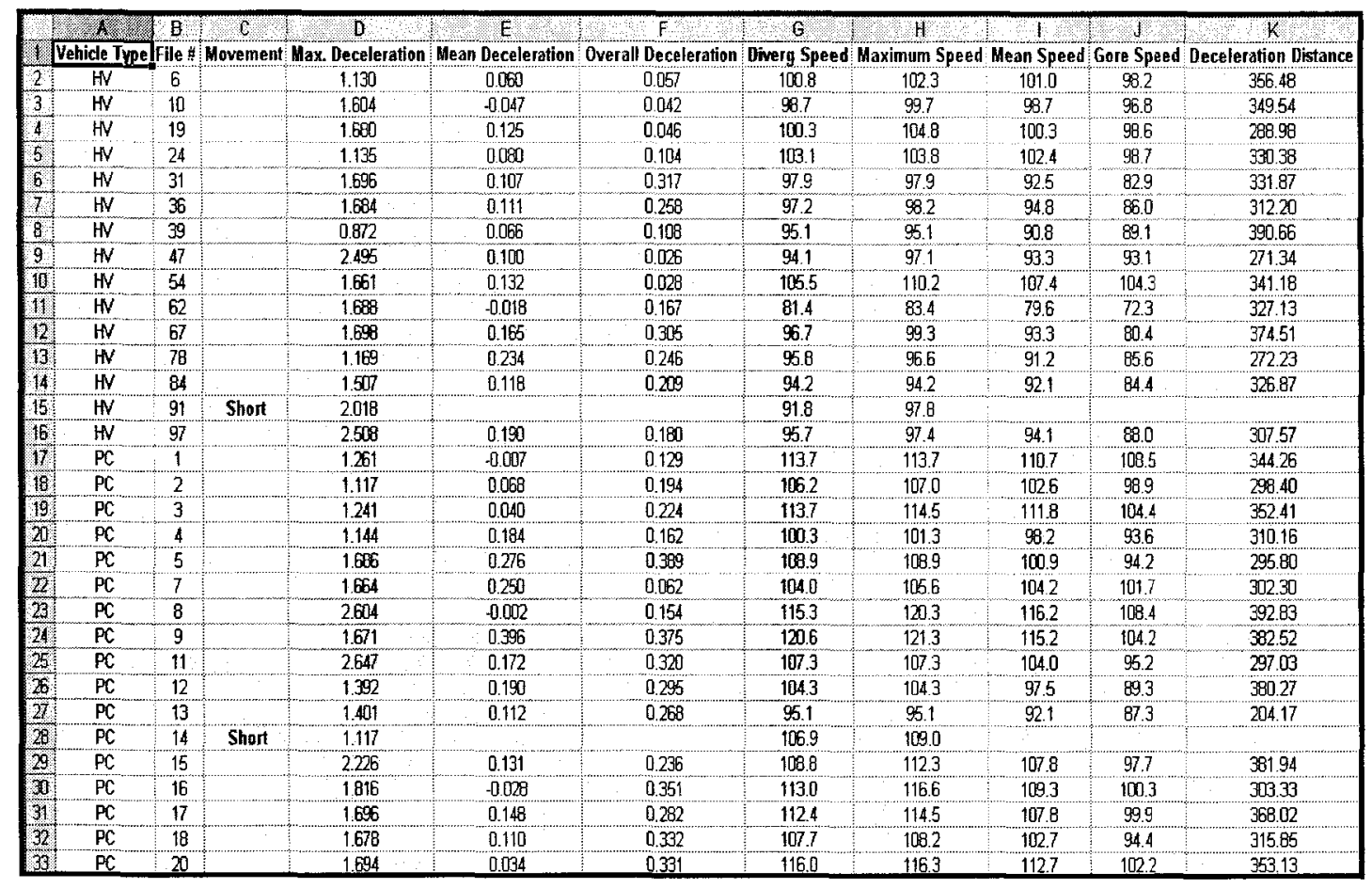

Figure 3.10: Analysis Parameters Summary for all Targeted Vehicles, Carp (E-NS)

\begin{tabular}{|c|c|c|c|c|c|c|c|c|c|c|c|}
\hline & xis & B & $c$ & (1) & $E$ & $F$ & 6 & 17 & 1 & I! & $K$ \\
\hline 1 & Vehicle Iype & File \# & Movement & Max. Deceleration & Mean Deceleration & Overali Deceleration & Initial Speed & Haximum Speed & Mean Speed: & Gore Speed & Deceleration Distance \\
\hline 2 & $\mathrm{HV}$ & 32 & & 2.927 & 0.029 & 0.356 & 118.9 & 120.0 & 114.7 & 95.9 & 544.22 \\
\hline 3 & $\mathrm{HN}$ & 33 & & 2.237 & 0.049 & 0.342 & 94.4 & 94.9 & 86.5 & 76.9 & 344.24 \\
\hline 4 & $H V$ & 49 & & 1.780 & 0.157 & 0.548 & 96.9 & 97.7 & 88.7 & 66.7 & 354.45 \\
\hline 5 & HV & 74 & & 2.459 & 0.097 & 0.120 & 94.5 & 101.4 & 95.3 & 84.8 & 581.62 \\
\hline 6 & WV & 97 & & 2271 & 0.289 & 0.192 & 889 & 88.9 & 82.5 & 73.8 & 501.03 \\
\hline 7 & $\mathrm{HV}$ & 101 & & 1.914 & 0.221 & 0.272 & 113.1 & 116.5 & 104.0 & 96.0 & 517.32 \\
\hline 9 & $\mathrm{HV}$ & 105 & & 2.272 & 0.246 & 0.262 & 96.4 & 96.4 & 90.1 & 82.5 & 371.73 \\
\hline 10 & WV & 106 & & 1.703 & 0.300 & 0.347 & 90.4 & 930 & 84.9 & 75.8 & 276.11 \\
\hline 11 & HN & 112 & & 2.409 & 0.474 & 0.302 & 1.06 .9 & 107.7 & 102.7 & 93.3 & 354.20 \\
\hline 12 & NV & 119 & & 1.702 & 0.153 & 0.121 & 71.9 & 71.9 & 66.7 & 60.4 & 494.48 \\
\hline 13 & N & 123 & & 2.253 & 0.239 & 0.265 & 93.6 & 942 & 90.1 & 787 & 380.70 \\
\hline 14 & $H N$ & 134 & & 2399 & 0.373 & 0.366 & 1119 & 1124 & 106.9 & 84.9 & 581.60 \\
\hline 15 & $\mathrm{PC}$ & 1 & Sh & 1527 & & & 104.3 & 104.3 & & & \\
\hline 16 & $\mathrm{PC}$ & 2 & & 1.697 & 0.515 & 0.513 & 86.7 & 86.7 & 80.6 & 68.4 & 214.25 \\
\hline 94 & PC & 132 & & 1.694 & 0.349 & 0.359 & 111.7 & 111.7 & 103.8 & 94.9 & 375.50 \\
\hline 95 & PC & 139 & $\mathbf{S h}$ & 2.397 & & & 1177 & 117.8 & & & \\
\hline 96 & PC & 44 & $\mathrm{ML}$ & 1135 & 0.018 & 0.116 & 93.3 & 98.2 & 95.9 & 97.6 & 276.13 \\
\hline 97 & PC & 80 & 网 & 2.241 & 0.321 & 0.063 & 1019 & 101.9 & 98.4 & 99.4 & 301.16 \\
\hline 98 & PC & 130 & $\mathrm{ML}$ & 1684 & 0.248 & 0.074 & 1300 & 130.0 & 129.5 & 128.7 & 175.55 \\
\hline 99 & PC & 13 & D & 2.825 & 0.656 & 0.469 & 105.6 & 105.7 & 92.8 & 80.0 & 400.87 \\
\hline (16) & $P C$ & 16 & D & 2513 & 0.263 & 0.558 & 129.8 & 129.8 & 120.4 & 95.4 & 553.28 \\
\hline (101) & $P C$ & 19 & D & 2.49 & 0.623 & 0.698 & 105.7 & 105.8 & 100.0 & 835 & 233.81 \\
\hline 102 & PC & 21 & D & 3.161 & 0.237 & 0.494 & 100.8 & 1000.8 & 89.8 & 80.7 & 285.56 \\
\hline 10 & $\mathrm{PC}$ & 23 & D & 2256 & 0.376 & 0.329 & 107.5 & 108.1 & 102.3 & 94.1 & 269.30 \\
\hline 104 & PC & 31 & D & 2.259 & 0.088 & 0.367 & 96.8 & 98.5 & 939 & 82.1 & 282.30 \\
\hline 105 & PC & 35 & D, Sh & 2060 & & & 107.6 & 108.1 & & & \\
\hline 435 & PC & 135 & D & 1.659 & 0.155 & 0.258 & $\overline{121.1}$ & 121.1 & 1160 & 108.6 & 428.79 \\
\hline \multicolumn{12}{|l|}{136} \\
\hline 137 & & Sh $=$ & Shont & & & & & & & & \\
\hline 138 & & $M L=$ & Merged Let & & & & & & & & \\
\hline 139 & & $D=$ & Diverging fror & $m$ Right-Lane & & & & & & & \\
\hline
\end{tabular}

Figure 3.11: Analysis Parameters Summary for all Targeted Vehicles, Greenbank (E-NS) 
Further adjustments were performed for converting signs associated with the calculated deceleration/acceleration rates (i.e. maximum, mean, and overall deceleration rates). Therefore, for the purpose of this study, a deceleration value having a negative sign means an acceleration rate. At that point, all the speed profiles collected on the freeway thirteen diverge areas (the deceleration SCLs and the adjacent right lanes) were ready to proceed to the next phase of this study.

\subsection{GEOMETRIC DATA}

The geometric data that were required to be part of the database gathered for completing this research were compiled from the geometric plans provided by the Ontario Ministry of Transportation (MTO). The supplied plans were a printout of the Engineering and Title Records (ETR) for all projects implemented on Highway 417. During the site investigation process, it was necessary to validate the plans with the existing conditions of the Highway to confirm they are all up to date.

Nevertheless, there was a newly constructed interchange at the Castlefrank Road between the Eagleson Road and the Terry Fox Drive. The Castlefrank interchange contained two ramps, one off-ramp (E-NS) and another on-ramp (NS-E). These two ramps of the Castlefrank Rd. interchange were connected to two other ramps $\mathrm{N}-\mathrm{W}$ and W-NS of the following interchange (Eagleson Rd.) through two auxiliary SCLs, respectively. At time of speed data collection phase of this research, Morrison Hersfield was in charge of the Castlefrank interchange and to the renovation of the Castlefrank pedestrian overpass. Therefore, Morrison Hersfield was contacted for an attempt to obtain a copy of the existing Highway conditions. In fact, Morrison Hersfield cooperated 
in providing a copy of the new plans for that newly constructed section of the Highway. Furthermore, Morrison Hersfield provided the vertical offset, measured from the pavement surface to the top of bridge deck of the newly constructed Castlefrank pedestrian overpass, as previously mentioned in Section 3.2.

\subsubsection{Geometric Plan Description}

Figure 3.12 and Figure 3.13 show two map samples of the ETR maps provided by MTO. The printed MTO plans were used to extract the required geometric data for the fulfilment of this research. The information that were required for this study included lengths of deceleration SCLs, transition lengths (from end of SCLs to controlling rampcurves), divergence angle at the gore area, ramp width, lengths of freeway segments (for safety performance modelling), and number of freeway basic lanes. Some geometric plans were found to have the edge of the pavement and lane marking, as the sample plan shown in Figure 3.12, and those were ideal to extract the required lengths and other geometric features. However, there were other geometric plans that only contained the general pavement boundaries with no lane markings as shown in the sample in Figure 3.13 . 


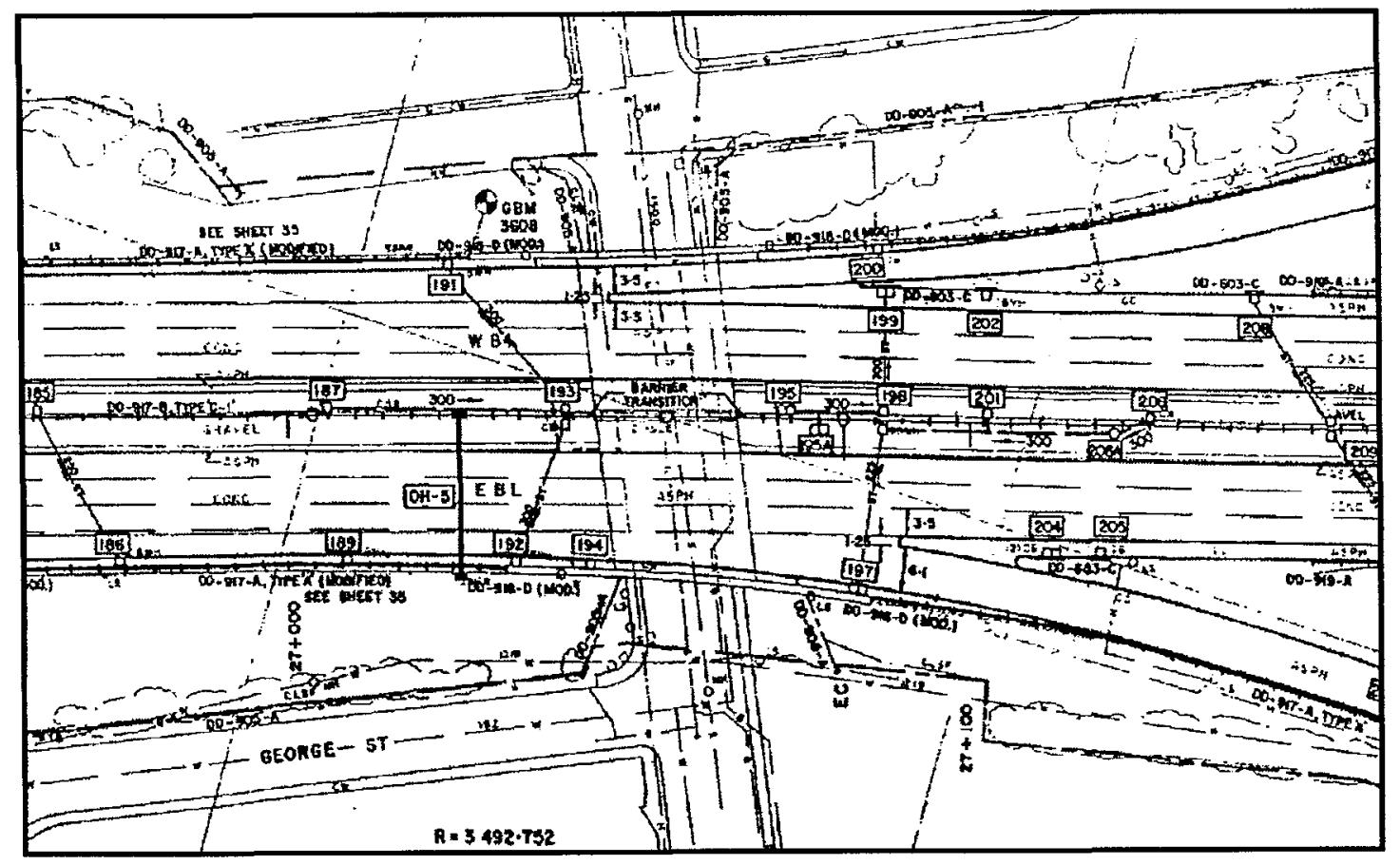

Figure 3.12: Sample of ETR Map - With Marking, MTO

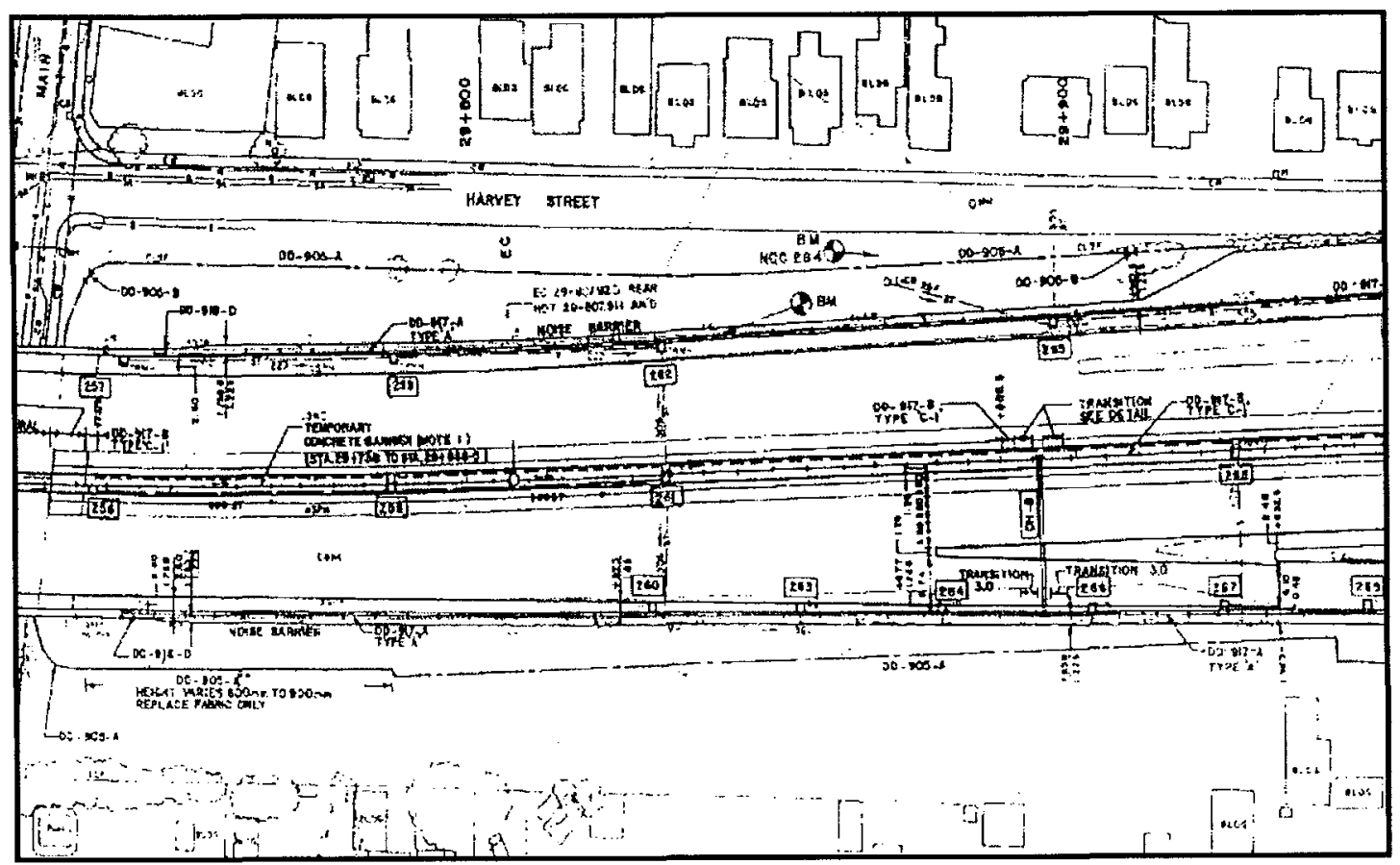

Figure 3.13: A Sample of ETR Map - Without Marking, MTO 


\subsubsection{Measurement of Geometric Features}

Different geometric features were extracted from the MTO plans. The lengths of the selected freeway deceleration SCLs, in addition to the transition lengths, ramp grade and width, the divergence angle between the exit-ramp and the freeway right most lane, and finally the number of basic freeway lanes were all extracted to study their effect on driver behaviour on freeway diverge areas. Next is a description of how each of these geometric features was extract from the MTO plans.

\subsection{2.a Length of Speed-Change Lane}

According to the geometric design guides, the lengths of limited length type deceleration SCLs were measured from the point where the pavement edge of the freeway right most through lane intersects with the pavement edge of the deceleration SCL, to the point where the two pavement edges of the freeway right lane and off-ramp are 1.25 meters apart. For extended length type deceleration SCLs, the lengths were measured from a point where the freeway mainline and the on-ramp pavement edges are separated by 1.25 meters to a similar point at the following off-ramp (where the two painted pavement edges of the mainline and the off-ramp lanes are 1.25 meters apart).

\subsection{2.b Transition Length}

The transition lengths were measured from the end of the freeway deceleration SCL (the 1.25 m marking separation) to the beginning of controlling off-ramp curve at the physical nose of the exit terminal. 


\subsection{2.c Ramp Grade and Width}

The ramp widths of the off-ramp lane(s) were directly extracted form the ETR geometric plans or were measured on the geometric plans using a ruler. In addition, during site investigation process, ramp grades were determined as an upgrade or downgrade depending on the inclination and slope direction of the ramp in accordance to its geometric structure.

\subsection{2.d Number of Basic Freeway Lanes}

The number of basic lanes of the freeway was simply counted in the field or on the geometric plans for the different sections of the freeway. Extended deceleration SCLs were not considered part of the freeway basic lanes.

\subsection{2.e Divergence Angle}

The divergence angles were measured from the geometric plans at the physical nose of the exit terminal using a protractor. The angles' vertex were determined as the point where the edge of the freeway right most through lane intersects with the extension of the edge of the exit-ramp lane at the gore area.

\subsection{2.f Lengths of Freeway Segments}

The lengths of freeway segments were measured using the same criterion of the deceleration SCLs' length determination, but with respect to the following and the preceding freeway segment. The lengths of the freeway segments were specifically measured for the purpose of safety performance. For that reason, lengths of freeway 
through segments in addition to the following deceleration SCLs and lengths of freeway deceleration SCLs only were measured and prepared for use in the analysis of safety performance.

\subsection{TRAFFIC DATA}

The study's primary objective was to develop operational and safety performance predictive models to help evaluate the drivers' behaviour on freeway diverge areas. Accordingly, two different traffic data sets were collected as part of the database gathered for the purpose of this research. The traffic volumes recorded in the field, using a video camera during the speed data collection process, were used as part of the database prepared to examine the driving performance to the prevailing traffic conditions. In addition, the average annual daily traffic (AADT) volumes, in relation to the collision data prepared for the safety performance modelling, were also used as part of the research database. Presented next is a description of the equivalent hourly traffic volumes followed by an explanation of the AADT data used for safety performance in this study.

\subsubsection{Equivalent Hourly Traffic Volumes}

The prevailing traffic volumes on all the sites selected for the purpose of this study were recorded using a video camera during the speed data collection process. These videos were used for later traffic count on both the freeway deceleration SCLs and the adjacent right lanes. During the speed data collection phase, the prevailing traffic volumes were recorded for periods of 30 to 60 minutes (the same time it took to collect the speed data on the respective site). The traffic count process incorporated counts for 
passenger cars (PC), heavy vehicles (HV), and recreational vehicles (RV) for different vehicle movements on the freeway diverge areas (i.e. changing-lane manoeuvres).

The equivalent hourly traffic volumes (in passenger cars per hour per lane $(\mathrm{pc} / \mathrm{h} / \mathrm{ln}))$ were obtained and calculated as the summation of passenger cars and the equivalent PC volumes for heavy and recreational vehicles counted during the playback of the recorded DVDs and then converted to hourly volumes. The HCM (2000) recommends the use of heavy vehicle (HV) and recreational vehicle (RV) adjustment factors for level terrain of 1.5 and 1.2 , respectively. These factors were used for calculating the equivalent traffic volumes. For DVDs that were recorded for less than 60 minutes, the equivalent traffic counts were then multiplied by the duration of the DVD (in minutes) and divided by sixty to obtain the equivalent hourly traffic volumes. Moreover, the heavy vehicle percentages incorporated into the traffic counts were calculated and summarized for the study sites. Section 4.1.5, in the next chapter, provides a detailed description of the equivalent hourly traffic volumes and the vehicle classification on the selected freeway deceleration SCL sites.

\subsubsection{Average Annual Daily Traffic (AADT)}

In addition to the equivalent hourly traffic volumes mentioned earlier, the traffic volumes of Highway 417 that have been summarized by Sarhan (2004) were used as part of the data prepared for the purpose of safety performance. The traffic volumes on the freeway mainline and ramps of Highway 417, collected from the MTO traffic volume database for years 2000 and 2002, consisted of two data sets. One data set contained both the peak-hour and the AADT volumes, while the other data set contained only the peak- 
hour volumes. Afterwards, Sarhan (2004) converted the peak-hour volumes to AADT using a conversion factor of $9.5 \%$, which was found to be very close to the typical value of $9.3 \%$ for urban roads as mentioned in the HCM (2000). Hence and for the purpose of this study, the peak-hour traffic volumes and the AADT data, associated with the selected thirteen freeway diverge areas, were extracted and prepared for use in the safety performance modelling attempts.

\subsection{COLLISION DATA}

The five-year period (1998-2002) collision data, collected and used by Sarhan (2004) for developing explicit safety performance models on freeway merge and diverge areas, were used to extract the collision data relevant to the sites selected in this study. Likewise, the prepared collision data were used in safety performance activities. Explanation of the source of the collision data and its preparation followed by the analysis parameter selected for safety performance modelling are presented next.

\subsubsection{Collision Data Source and Preparation}

The collision data, collected by Sarhan (2004) from the City of Ottawa, classified the collisions according to the type of the collision as fatal, injury, or property damage only (PDO). Afterwards, Sarhan (2004) formed two main classes namely: "Total Collisions" and "Equivalent Property Damage Only (EPDO) Collisions" for safety modelling process. The "Total" class was simply the summation of all collisions regardless of the type of collision. On the other hand, the "EPDO" class was the summation of weighted collision frequencies based on the type of collision using 
recommended weights of 1,6 , and 12 for PDO, injury, and fatal collisions, respectively. Sarhan (2004) attempts for developing safety models using the second class (EPDO) were not successful. Therefore, and for the purpose of this research, only total collision frequencies were used in safety performance modelling attempts.

\subsubsection{Analysis Parameter for Safety Performance}

As mentioned earlier, the collision data summary, prepared by Sarhan (2004), was used to extract the collision data associated with the freeway diverge areas selected for this study. The collision data were prepared to help evaluate the effect of freeway geometry, traffic exposure, and speed data on collision prediction on freeway diverge areas using two safety parameters. These safety parameters (collision data classes) were performed for safety performance modelling attempts on freeway diverge areas in this study. The following is a description of the two safety parameters prepared for use in safety performance modelling attempts:

1) Collisions on Freeway Through-Segment and the Deceleration SCL, $\left(\mathrm{Col}_{\text {ThDec }}\right)$

$\mathrm{Col}_{\text {ThDec }}$ is the summation of collisions on the freeway through segment plus the following deceleration SCL, excluding the preceding terminal.

2) Collisions on Freeway Deceleration SCL, $\left(\mathrm{Col}_{D e c}\right)$

$\mathrm{Col}_{D e c}$ is the number of collisions on the freeway deceleration SCL. 


\section{CHAPTER FOUR \\ 4.0 DESCRIPTIVE ANALYSIS}

This chapter covers a detailed descriptive analysis summary of the database collected in this study. Various frequency distribution arrangements are presented to describe the basic features of the collected data after processing. Section 4.1 presents the number of vehicles targeted at the selected sites. Section 4.2 presents the vehicle movement distributions on freeway speed-change lanes (SCLs) and the adjacent right lanes (RLs) for the study sites. Then, Section 4.3 presents vehicle speed profiles created for vehicles targeted on the freeway deceleration SCLs selected for the study. Descriptions of the geometric features of the selected sites are presented in Section 4.4. Section 4.5 presents the calculated equivalent hourly traffic volumes for selected sites as well as the average annual daily traffic (AADT) volumes prepared for the purpose of safety performance. Then, Section 4.6 presents a description of the collisions occurred on the selected sited during the five-year period 1998-2002. Finally, Section 4.7 provides a descriptive summary of the aggregated driver behaviour measures on the selected sites including the speeds (diverge, gore, and maximum), deceleration rates (overall, mean, and maximum), and the effective deceleration distances on the freeway deceleration SCLs as well as the mean speeds on the freeway adjacent RLs on selected sites.

\subsection{NUMBER OF TARGETED VEHICLES}

As mentioned in the Chapter 3.0, eleven interchange on Highway 417 with thirteen exit terminals have been selected for the purpose of this study to collect actual speed data on both the freeway speed-change lanes and the adjacent right lanes. The 
number of vehicles targeted on the freeway deceleration SCLs and the adjacent RLs varied among the selected sites. Figure 4.1 shows the distribution of the total vehicles (combined PC and HV) targeted on site for the thirteen exit terminals, excluding the speed data files that were deleted during the data processing phase. In Figure 4.1, the first seven deceleration SCLs (counted from left) are limited length type SCLs, while the last six sites are extended length type deceleration lanes.

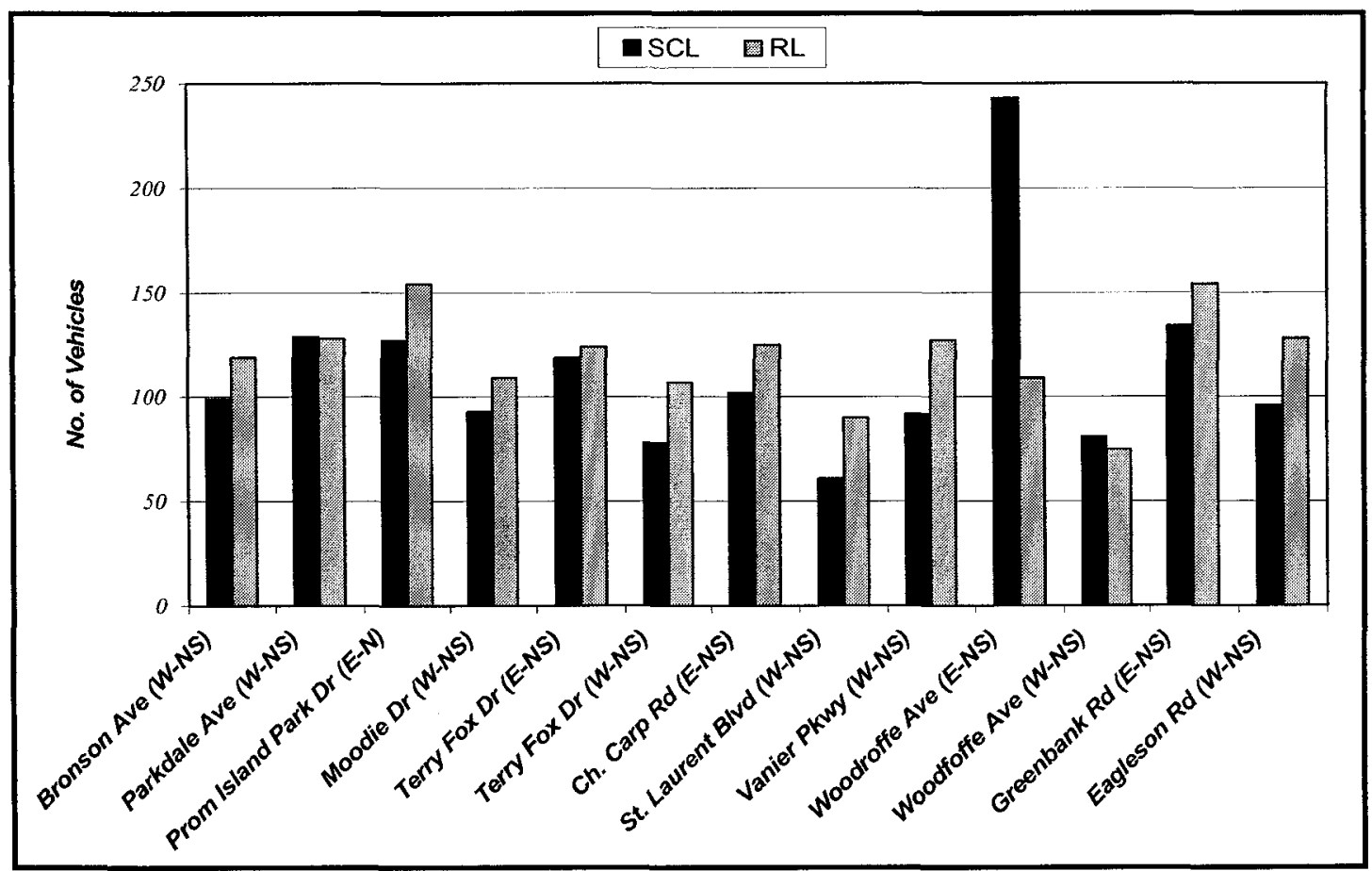

Figure 4.1: Frequency Distribution of Number of Vehicles on SCL and RL

The number of targeted vehicles on both the freeway deceleration SCLs and the adjacent RLs at all the selected sites, used for collecting the actual speed data in this study, ranged from 60 to 155 vehicles, except for one site - Woodroffe (E-NS). As shown in Figure 4.1, the total number of targeted vehicles on Woodroffe Ave (E-NS) SCL was 
as high as 243 , with 109 vehicles targeted on its adjacent RL. Such a large number of targeted vehicles on Woodroffe (E-NS) SCL was due to the special geometric feature this site comprises as compared to other sites. Clarification of the geometric nature of Woodroffe (E-NS) SCL is provided in the following sections.

\subsection{VEHICLE MOVEMENT DISTRIBUTIONS ON RL AND SCL}

As mentioned in the previous chapter, in order to record speed data for vehicles travelling on the freeway deceleration SCLs and the adjacent RLs at the selected sites, two researchers (operators) worked together side-by-side for this purpose. The first operator targeted the vehicles travelling on the freeway deceleration SCL, while the second operator targeted the vehicles travelling on the freeway adjacent RL. The distribution of vehicles and their movements on the thirteen freeway deceleration SCLs and the adjacent RLs were created to graphically illustrate the traffic movements on the freeway diverge areas. Figure 4.2 and Figure 4.3 are two samples of vehicle distribution and their movements on two different types of exit terminals, Bronson W-NS and St. Laurent W-NS, respectively. Bronson W-NS is a limited (taper) length type deceleration SCL, whereas St. Laurent W-NS is an extended length type SCL. The figures illustrating the distributions of vehicle movement for all the thirteen sites used in this study are available in Appendix A. It should be noted that the balance of targeted vehicles in the vehicle movement distribution figures is not step because of outliers (presented in Section 4.7.2) and/or short speed profiles. 


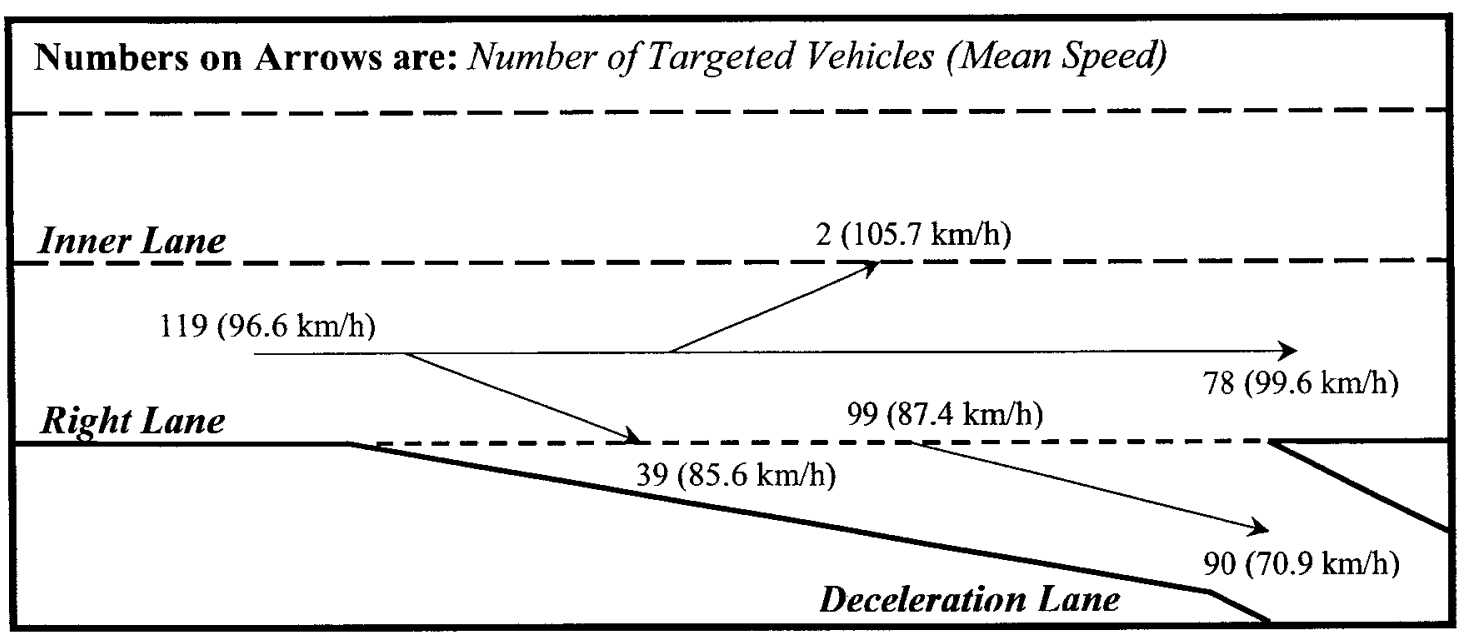

Figure 4.2: Sample Distribution of Vehicles and their Movement on Bronson (W-NS)

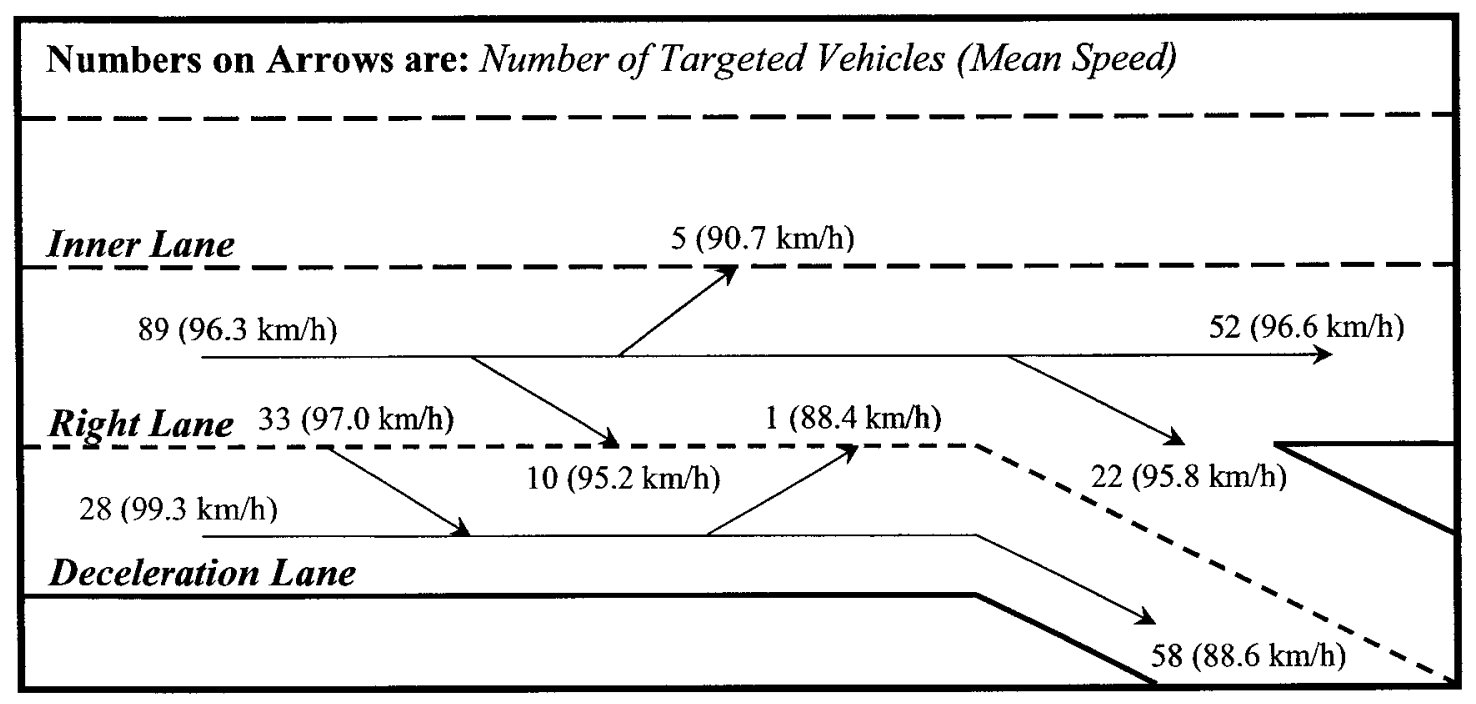

Figure 4.3: Sample Distribution of Vehicles and their Movement on St. Laurent (W-NS)

The arrows on the drawings indicate the direction of travel for all possible traffic movements. As shown in the figures, for the traffic streams on freeway limited length type SCLs, the information between the brackets indicate the mean diverge speed and mean gore speed of all vehicles targeted diverging onto the SCL and exiting the freeway. 
As mentioned earlier, the vehicles diverged from the freeway RL onto the SCL that had short speed profiles (ended before the end of the relevant SCL) have no gore speeds. Concerning the traffic streams on freeway extended length type SCLs, the information between the brackets indicate the mean initial speed, mean diverge speed, mean merge speed and mean gore speed for targeted vehicles that already occupied the SCL, diverged onto the SCL, merged left onto the adjacent RL, and exited the freeway, respectively.

On the other hand, for the traffic streams on freeway RLs, the numbers between the brackets indicate the mean average speed, mean diverge speed, and mean merge speed for vehicles targeted upstream the freeway diverge area and continued through until the limits of the exit ramp gore, diverged onto the SCL, and merged left onto the freeway inner lane, respectively. Finally, for cases of deceleration SCLs of weaving type "B", where the left hand-lane of the two-lane exit ramps permits vehicles (desired to depart) to directly exit the freeway, the number between the brackets indicate the mean exit speed of those vehicles exiting the freeway using the left-hand lane of a two-lane exit ramp (see Figure 4.3). It should be noted that these exit speeds were not recorded at the $1.25 \mathrm{~m}$ separation (end of SCL) and hence, they are not gore speeds.

For example, in Figure 4.3 , the total number of vehicles targeted upstream the freeway diverge area of St. Laurent (eastbound direction) was 89 with a mean average speed of $96.3 \mathrm{~km} / \mathrm{h}$. Out of those 89 vehicles, 52 vehicles continued along the freeway $\mathrm{RL}$ with a mean average speed downstream the freeway diverge area (near the physical nose) of $96.6 \mathrm{~km} / \mathrm{h}$. The other 37 vehicles were changed lanes as follows:

- Ten vehicles diverged onto the SCL with a mean diverge speed of $95.2 \mathrm{~km} / \mathrm{h}$. 
- Five vehicles merged left onto the freeway inner lane with a mean merge speed of $90.7 \mathrm{~km} / \mathrm{h}$.

- Twenty-two vehicles exited the freeway via the left-hand lane of the two-lane exit ramp with a mean exit speed of $95.8 \mathrm{~km} / \mathrm{h}$.

Moreover, for the vehicles targeted on St. Laurent SCL in Figure 4.3, there were 29 vehicles already traveling in the SCL and the mean initial speed of those vehicles was equal to $99.3 \mathrm{~km} / \mathrm{h}$. Among those vehicles, only one vehicle merged left onto the adjacent RL with a merge speed of $88.4 \mathrm{~km} / \mathrm{h}$. In addition, 33 vehicles were targeted while diverging from the freeway RL to the SCL with a mean diverge speed of $97.0 \mathrm{~km} / \mathrm{h}$. In the end, only 58 vehicles exited the freeway via the exit ramp lane connected to the deceleration SCL with a mean gore speed at the $1.25 \mathrm{~m}$ separation (end of the SCL) of $88.6 \mathrm{~km} / \mathrm{h}$. It should be noted that there were two vehicles that either had their profiles ended before the end of the St. Laurent SCL (i.e. short speed profiles) and therefore no gore speed was recorded at the $1.25 \mathrm{~m}$ separation or were determined as an outlier(s) as will be explained later in Section 4.7.

As mentioned earlier, Woodroffe (E-NS) had a unique geometric feature of two exit ramps in two separated directions (northbound and southbound). Figure 4.4 shows the Woodroffe (E-NS) SCL with the distribution of targeted vehicles and their movements as well as their mean speeds, as applicable. The Woodroffe E-NS is an extended length type deceleration SCL connecting the "Maitland Ave." and the "Woodroffe Ave." interchanges in the westbound direction. 


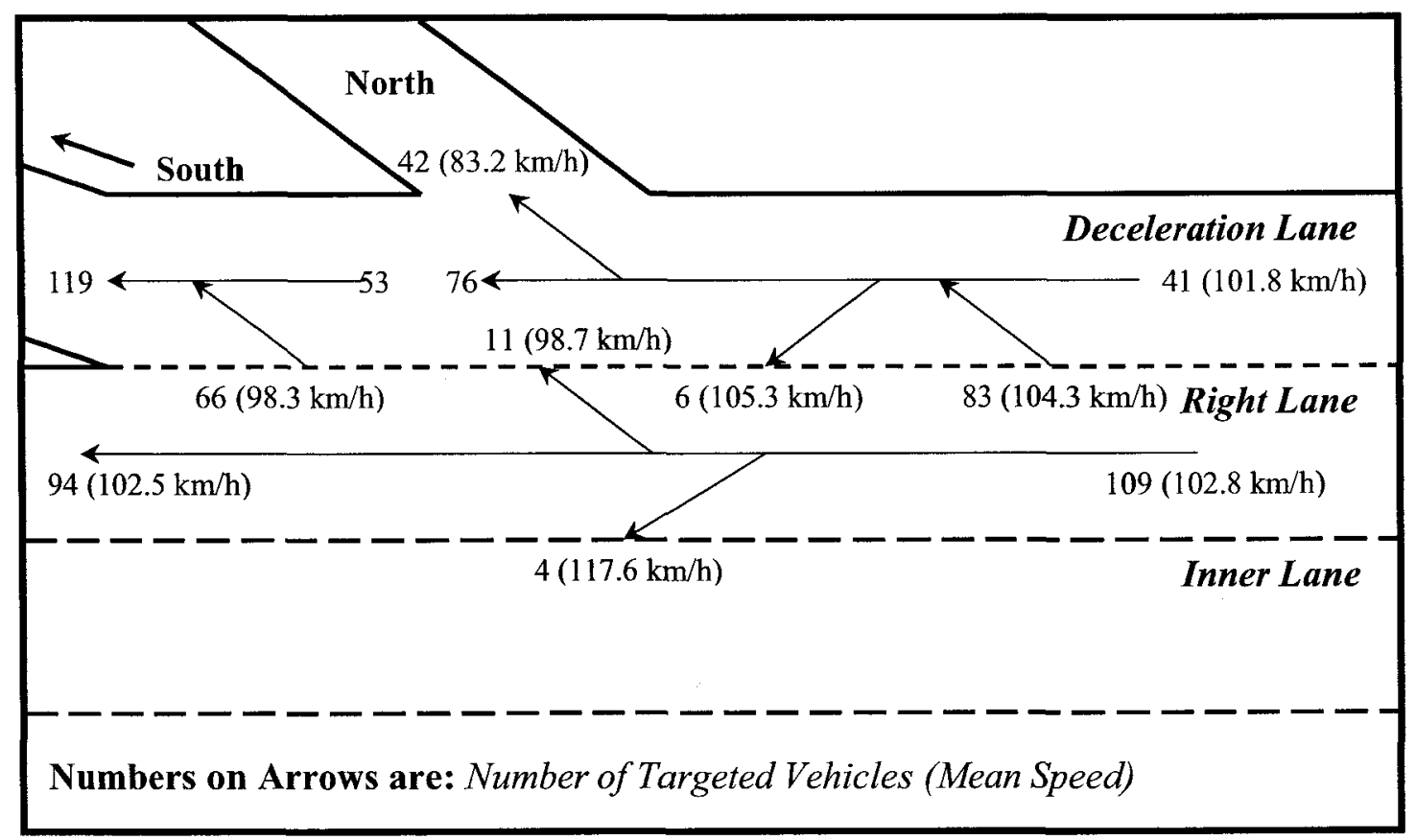

Figure 4.4: Sample Distribution of Vehicles and their Movements on Woodroffe (E-NS)

The speed data for 243 vehicles were recorded on Woodroffe (E-NS) SCL for two different segments along the Woodroffe (E-NS) SCL with respect to the north exit ramp; before the north exit ramp and after the north exit ramp, respectively. First, 124 vehicles (41 vehicles already occupying the SCL and 83 vehicles that diverged from the freeway RL onto the SCL), were targeted on Woodroffe (E-NS) SCL from a point upstream the freeway diverge area to a point in the proximity of the physical nose of the north exit ramp and were distributed as follows:

- Forty-two vehicles exited the freeway via the north exit ramp.

- Six vehicles merged left onto the freeway RL.

- Seventy-six vehicles continued on the SCL towards the south exit ramp.

It should be noted that the 76 vehicles that continued on the deceleration SCL and headed towards the south exit ramp had their speed profiles ended before the end of 
Woodroffe (E-NS) SCL and therefore their end operating speeds were not gore speed values. Second, 119 vehicles (53 vehicles already occupying the SCL plus 66 vehicles that diverged from the freeway $\mathrm{RL}$ ) were targeted from a point near the physical nose of the north exit ramp to a point near the physical nose of the south exit ramp. Because the operators' position on the "Woodroffe Ave" overpass was right over the painted nose of the freeway deceleration SCL at the south exit ramp, it was not possible to record gore speeds for the targeted vehicles exiting the freeway via the south exit ramp.

\subsection{VEHICLE SPEED PROFILES}

As mentioned in Chapter 3.0, the speed data collected on the freeway deceleration SCLs on the thirteen study sites were used to create vehicle speed profiles. In case of an extended length type SCL, the speed profile of a vehicle already occupying the targeted SCL begins with an initial speed and ends with a gore speed or a merge speed depending on whether that vehicle exited the freeway or merged left onto the freeway RL. The speed profile for a vehicle diverging from the freeway RL onto an extended or a limited length type SCL and exiting the freeway begins with a diverge speed and ends with a gore speed. On the other hand, in case of a limited length type SCL, a speed profile always begins with a diverge speed and ends with a gore speed only if that vehicle was targeted up to a point in the proximity of the physical nose. Consequently, short speed profiles end with insignificant speeds that were recorded before the $1.25 \mathrm{~m}$ separation (i.e. end of $\mathrm{SCL}$ ). For this reason, short speed profiles were only used in determining the diverge speed, the maximum speed reached on the $\mathrm{SCL}$, the maximum deceleration rate performed on the SCL, and the effective deceleration distance used for deceleration on 
the SCL (measured from point where such vehicle diverged onto the SCL to end of the SCL).

Figure 4.5 and Figure 4.6 show two speed profiles samples for all vehicles targeted (all vehicle movement) on Vanier W-NS, an extended length type SCL, and Bronson W-NS, a limited length type SCL, respectively. The figures showing the speed profiles for all thirteen deceleration SCLs used in this study are available in Appendix B. The point where the mainline and the ramp pavement edges are $1.25 \mathrm{~m}$ apart (end of the relevant SCL), the physical nose, and the beginning of the SCL (for limited length types only) were all indicated in each of these figures for easy reference.

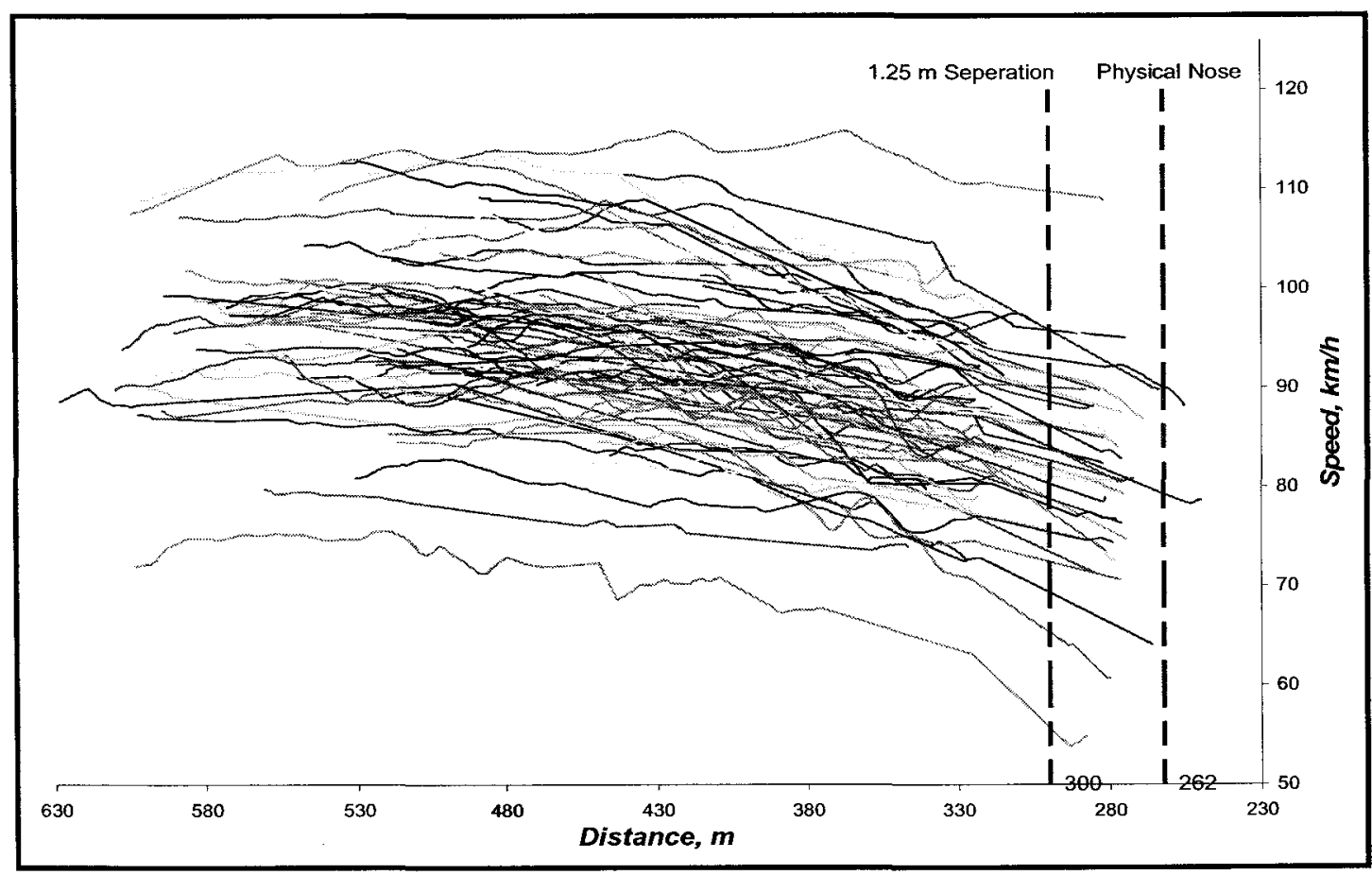

Figure 4.5: Speed Profiles of All Vehicle Targeted on Vanier Pkwy (W-NS) 


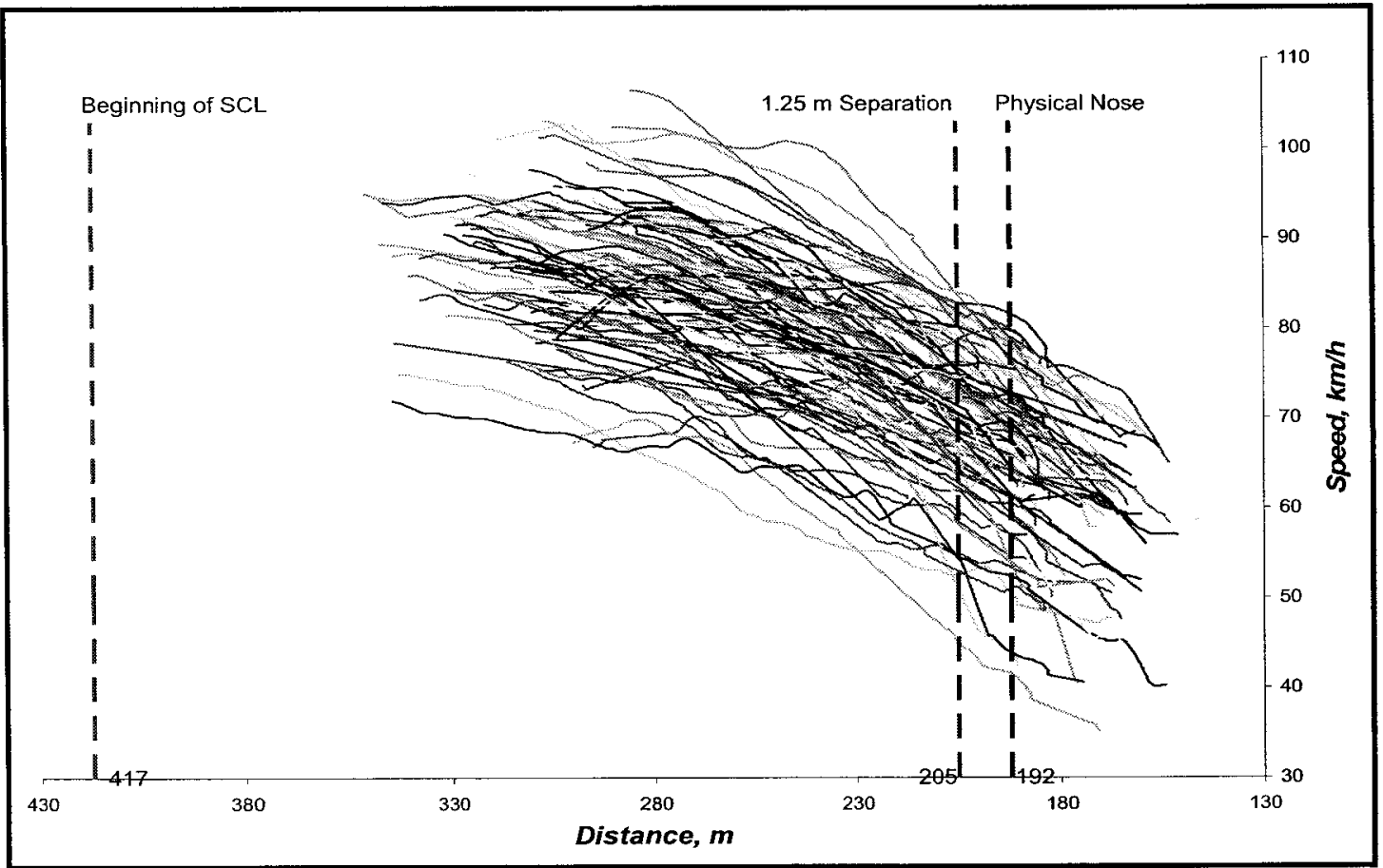

Figure 4.6: Speed Profiles of All Vehicle Targeted on Bronson Ave (W-NS)

As previously mentioned in Section 3.3.2.c, speed profiles were adjusted to only use the speed data readings between the beginning (for limited SCLs) and the end of the deceleration SCL in further analysis, whereas additional speeds recorded beyond the end of the deceleration relevant SCL were erased.

\subsection{GEOMETRIC FEATURES}

As mentioned earlier, the geometric data related to the selected study sites were also collected, and several geometric elements were extracted for each site as shown in Table 4.1. 
Table 4.1: Summary of Geometric Data for Exit Ramp Terminals Selected for this Study

\begin{tabular}{|c|c|c|c|c|c|c|c|c|c|c|}
\hline \multirow{2}{*}{ IC \# } & \multirow{2}{*}{ SCL Name } & \multirow{2}{*}{$\begin{array}{l}\text { Weaving } \\
\text { Type }\end{array}$} & \multicolumn{2}{|c|}{ Length, m } & \multirow{2}{*}{$\begin{array}{l}\text { SCL } \\
\text { Type }\end{array}$} & \multirow{2}{*}{$\begin{array}{l}\text { Ramp } \\
\text { Grade }\end{array}$} & \multirow{2}{*}{$\begin{array}{c}\text { Ramp } \\
\text { Width, m }\end{array}$} & \multirow{2}{*}{$\begin{array}{c}\text { Freeway } \\
\text { Basic } \\
\text { Lanes }\end{array}$} & \multirow{2}{*}{$\begin{array}{l}\text { Divergence } \\
\text { Angle, deg. }\end{array}$} & \multirow{2}{*}{$\begin{array}{l}\text { Ramp Advisory } \\
\text { Speed, km/h }\end{array}$} \\
\hline & & & SCL & Transition & & & & & & \\
\hline $121 \mathrm{~A}$ & $\begin{array}{c}\text { Bronson } \\
\text { (W-NS) }\end{array}$ & A & 212 & 13 & Taper & Down & 6.1 & 4 & 11 & 40 \\
\hline 122 & $\begin{array}{c}\text { Parkdale } \\
\text { (W-NS) }\end{array}$ & A & 179 & 41 & Taper & Down & 6.1 & 4 & 12 & 50 \\
\hline 123 & $\begin{array}{c}\text { Island Park } \\
(\mathrm{E}-\mathrm{N})\end{array}$ & A & 58 & 22 & Taper & Down & 6.8 & 4 & 18 & 40 \\
\hline 134 & $\begin{array}{l}\text { Moodie } \\
\text { (W-NS) } \\
\end{array}$ & A & 292 & 83 & Parallel & $\mathrm{Up}$ & 4.8 & 2 & 7 & 50 \\
\hline 140 & $\begin{array}{c}\text { Terry Fox } \\
\text { (E-NS) }\end{array}$ & A & 388 & 112 & Parallel & Up & 4.6 & 2 & 6.5 & 50 \\
\hline 140 & $\begin{array}{c}\text { Terry Fox } \\
\text { (W-NS) }\end{array}$ & A & 446 & 124 & Parallel & Up & 4.6 & 2 & 6 & 60 \\
\hline 144 & $\begin{array}{c}\text { Carp Rd } \\
\text { (E-NS) }\end{array}$ & A & 430 & 49 & Parallel & $\mathrm{Up}$ & 5.2 & 2 & 4 & 70 \\
\hline 115 & $\begin{array}{c}\text { St. Laurent } \\
\text { (W-NS) }\end{array}$ & B & 873 & 96 & Extended & Down & 7.5 & 3 & 5 & 60 \\
\hline 117 & $\begin{array}{c}\text { Vanier } \\
(\mathbf{W}-\mathbf{N S})\end{array}$ & $B$ & 707 & 38 & Extended & Down & 7.5 & 3 & 12 & 60 \\
\hline 127 & $\begin{array}{c}\text { Woodroffe } \\
\text { (E-NS) }\end{array}$ & A & 1320 & 59 & Extended & $\mathrm{Up}$ & 4.8 & 3 & 5 & 40 \\
\hline 127 & $\begin{array}{c}\text { Woodroffe } \\
(\text { W-NS) }\end{array}$ & A & 1358 & 63 & Extended & Up & 4.8 & 3 & 7 & 60 \\
\hline 129 & $\begin{array}{c}\text { Greenbank } \\
\text { (E-NS) }\end{array}$ & $B$ & 1047 & 38 & Extended & Up & 7.5 & 3 & 12 & 50 \\
\hline 138 & $\begin{array}{c}\text { Eagleson } \\
\text { (W-NS) }\end{array}$ & A & 697 & 115 & Extended & Up & 4.8 & 2 & 7 & 40 \\
\hline
\end{tabular}


From the information presented in Table 4.1, the lengths of extended and limited deceleration SCLs varied from one location to another for all the study sites. The lengths of limited length type SCLs were measured from the point where the painted pavement right-edge of the SCL intersects with the mainline pavement edge to the point where the mainline and exit ramp pavement edges are at a distance of $1.25 \mathrm{~m}$. The lengths of the limited length type deceleration SCLs (including taper) ranged from $58 \mathrm{~m}$ to $446 \mathrm{~m}$ with a mean value of $286.43 \mathrm{~m}$ and a standard deviation of $144.73 \mathrm{~m}$. On the other hand, the lengths of extended length type SCLs consider the distance connecting the preceding and the following bounding ramps and were measured between the two points where the two pavement edges of the mainline and the ramp are $1.25 \mathrm{~m}$ apart at the painted nose of the two bounding ramps. The lengths of the extended length type deceleration SCLs ranged between $697 \mathrm{~m}$ and $1358 \mathrm{~m}$ with a mean value of $1000.33 \mathrm{~m}$ and a standard deviation equal to 292.08 meters. Table 4.2 summarizes the descriptive statistics values of the lengths for both limited and extended deceleration SCLs, as well as for transition lengths, ramp widths, and divergence angles.

Table 4.2: Descriptive Summary of Geometric Data for Exit Terminals

\begin{tabular}{|c|c|c|c|c|c|}
\hline Statistics & $\begin{array}{c}\text { Limited } \\
\text { Length, } \mathbf{m}\end{array}$ & $\begin{array}{c}\text { Extended } \\
\text { Length, } \mathbf{m}\end{array}$ & $\begin{array}{c}\text { Transition } \\
\text { Length, m }\end{array}$ & $\begin{array}{c}\text { Ramp } \\
\text { Width, m }\end{array}$ & $\begin{array}{c}\text { Divergence } \\
\text { Angle, deg. }\end{array}$ \\
\hline Minimum & 58 & 697 & 13 & 4.6 & 4 \\
\hline Maximum & 446 & 1358 & 124 & 7.5 & 18 \\
\hline Mean & 286.43 & 1000.33 & 65.62 & 5.75 & 8.65 \\
\hline SD & 144.73 & 292.08 & 36.92 & 1.19 & 4.03 \\
\hline
\end{tabular}

During the speed data collection process, the transition pavement distances, connecting the end of the deceleration SCLs to the beginning of controlling off-ramp 
curves, were observed to comprise high disparities among the selected sites and are most probably to affect the drivers' behaviour on the freeway diverge areas. As shown in Table 4.2, the measured transition lengths are distributed around their mean value of $65.62 \mathrm{~m}$ with a standard deviation of $36.92 \mathrm{~m}$ while ranging from a minimum value of 13 meters to a maximum value of 124 meters. In Table 4.2 , the widths of pavement ramps at the physical nose range from 4.6 to 7.5 meters with a mean width of $5.75 \mathrm{~m}$ and a standard deviation of $1.19 \mathrm{~m}$ for all sites. It should be noted that the widths of pavement ramps (the recovery areas) at the physical nose for two-lane exit-ramps attained a value of 7.5 meters as the case for St. Laurent (W-NS), Vanier (W-NS), and Greenbank (E-NS).

The divergence angle was another geometric feature that has been measured and summarized to study its effect on driver deceleration behaviour on freeway diverge areas. The values of the divergence angles for all selected sites varied between $4^{\circ}$ and $18^{\circ}$ with a mean divergence angle of $8.65^{\circ}$ and a standard deviation of $4.03^{\circ}$. From the divergence angle values shown in Table 4.1, the limited length type SCLs (combined taper and parallel) had a mean divergence angle of $9.21^{\circ}$ and a std. dev. of $4.79^{\circ}$. Taper length type SCLs engaged with higher divergence angles than those with parallel length type SCLs. On the other hand, the extended length type SCLs attained a lower range of divergence angles with a mean value of $8^{\circ}$ and a standard deviation of $3.22^{\circ}$.One more geometric element that is collected was the number of the freeway basic through lanes adjacent to the deceleration SCLs that also varied from one location to another (see Table 4.1). The advisory speeds posted on all thirteen exit ramps selected for this study were collected as shown in Table 4.1 . 
Finally, among the collected geometric data, there were three categorical variables; namely: the SCL configuration, the weaving type, and the ramp grade (Table 4.1). First, there were seven deceleration SCLs of limited length type and six extended length type deceleration SCLs. The limited length type deceleration SCLs consisted of three taper length type lanes and four parallel type lanes. Second, the ramp grades for the sites selected were classified into two categories, upgrade and downgrade. In Table 4.1, eight upgrade ramps and five downgrade ramps define the ramp grade distribution of all selected sites. Finally, the weaving type of the deceleration SCLs took two types as "A" and "B". There were three extended deceleration lanes of weaving type "B" at St. Laurent (W-NS), Vanier (W-NS), and Greenbank (E-NS), leaving the rest of the deceleration SCLs with weaving type "A" (see Table 4.1). It should be noted that the weaving type variable also represents the number of exit lanes. A freeway deceleration SCL of weaving type "A" has a one-lane exit ramp whereas a deceleration SCL of weaving type "B" has a two-lane exit-ramp.

\subsection{TRAFFIC VOLUMES}

\subsubsection{Equivalent Hourly Traffic}

As mentioned in Chapter 3.0, the equivalent hourly traffic volumes on the freeway diverge areas of the selected sites were estimated from the recorded DVD's on both deceleration SCL and the adjacent RL. Figure 4.7 and Figure 4.8 show the frequency distribution of equivalent hourly traffic volumes during the speed data collection on the freeway RLs and the deceleration SCLs, respectively. 


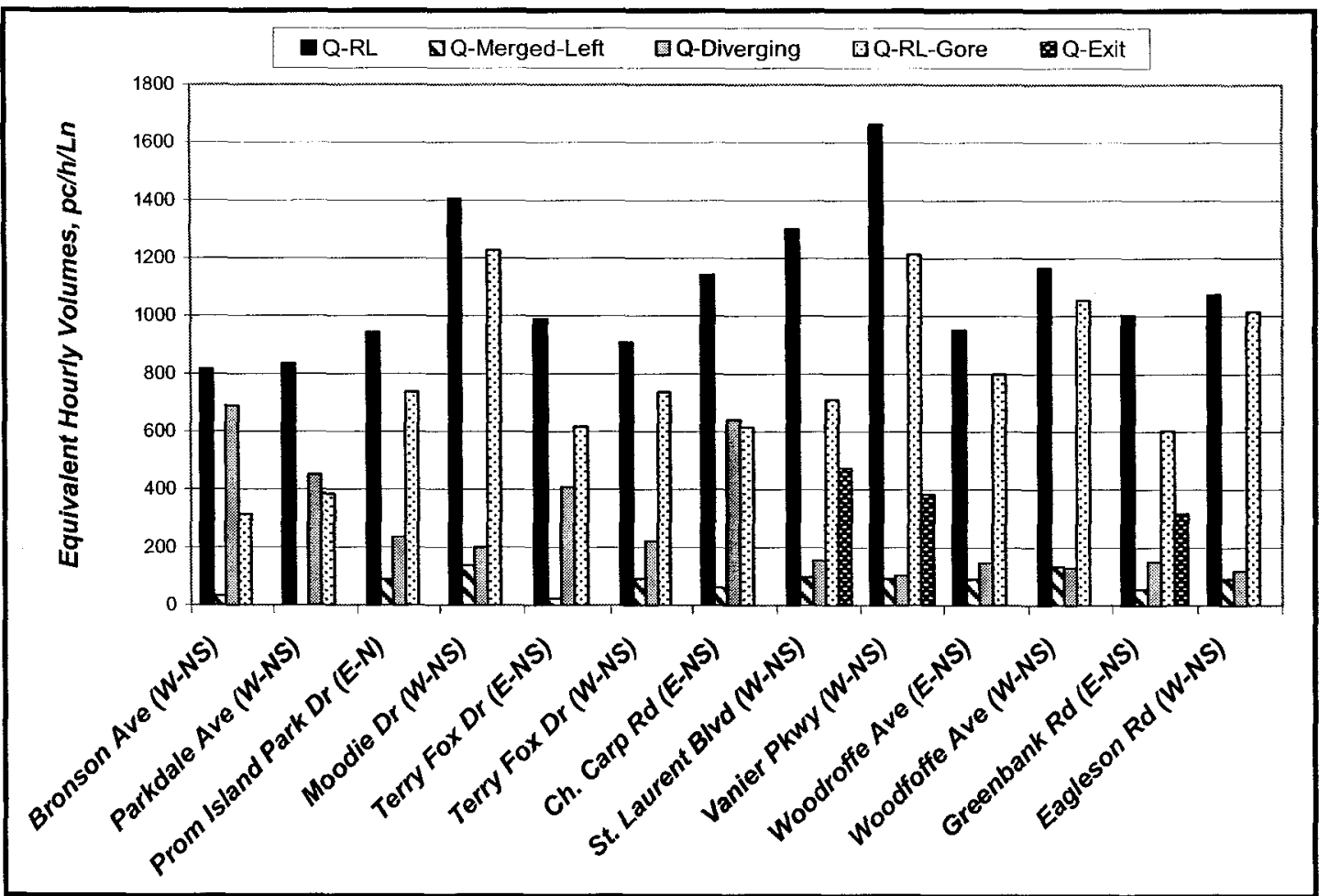

Figure 4.7: Distribution of Equivalent Hourly Traffic Volumes on RLs

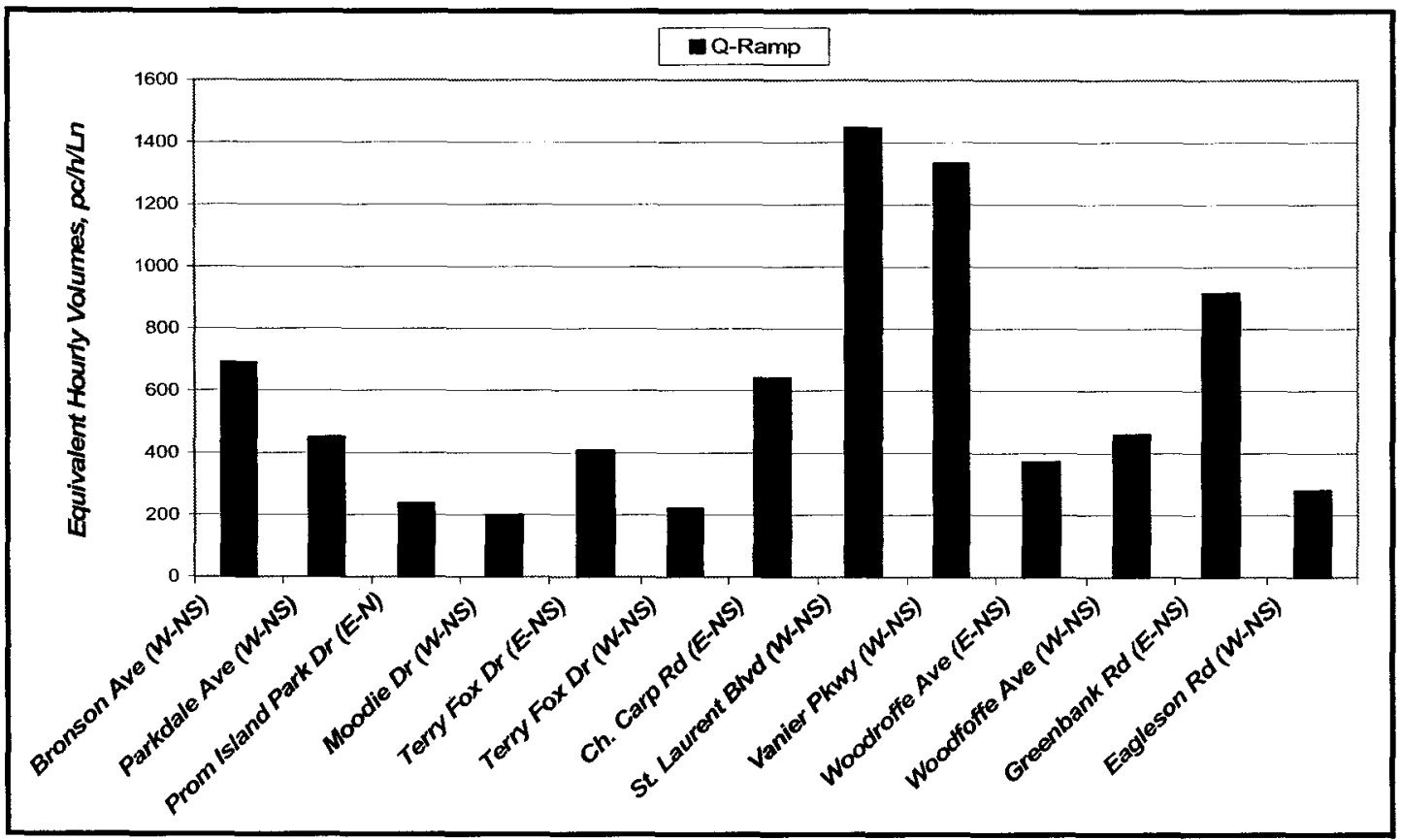

Figure 4.8: Distribution of Equivalent Hourly Traffic Volumes on SCLs 
The equivalent hourly volumes on the freeway RLs were calculated upstream and downstream the freeway diverge areas. The right lane upstream equivalent traffic volumes $\left(\mathrm{Q}_{R L}\right)$ included all vehicle movements (merged left onto the freeway inner lane, diverged onto the SCL, or continued through on the same RL), whereas the right lane downstream equivalent traffic volumes $\left(\mathrm{Q}_{R L \text {-Gore }}\right)$ represent the through vehicles only. The equivalent hourly traffic volumes for vehicles that merged left from the freeway RL onto the freeway inner lane ( $\left.Q_{\text {Merged-Left }}\right)$ as well as the equivalent hourly traffic volumes for vehicles that diverged from the freeway RL onto the freeway deceleration SCL ( $\left.Q_{\text {Diverging }}\right)$ were both calculated for all selected sites. Finally, the equivalent hourly traffic volumes for vehicles that exited the freeway via the left-hand lane of a two-lane exit ramp $\left(Q_{E x i t}\right)$ were calculated for all of St. Laurent (W-NS), Vanier (W-NS), and Greenbank (E-NS) sites. On the other hand, regarding the freeway SCLs, the equivalent hourly traffic volumes downstream the deceleration SCLs at the gore area were calculated for both limited and extended length type SCLs to represent the ramp traffic volumes $\left(Q_{\text {Ramp }}\right)$.

In Figure 4.7, the RL-upstream equivalent hourly traffic volumes $\left(\mathrm{Q}_{R L}\right)$ ranged from 816 to $1664 \mathrm{pc} / \mathrm{h} / \mathrm{ln}$ with a mean volume of 1093 and a standard deviation of 243.67, whereas the $\mathrm{RL}$-gore equivalent traffic volumes $\left(\mathrm{Q}_{R L \text {-Gore }}\right)$ ranged from 314 to $1228 \mathrm{pc} / \mathrm{h} / \mathrm{ln}$ and had a mean volume of $771 \mathrm{pc} / \mathrm{h} / \mathrm{ln}$ and a standard deviation of 286.56 $\mathrm{pc} / \mathrm{h} / \mathrm{ln}$. In addition, the average traffic volumes of the two RL-upstream and RL-gore equivalent traffic volumes were found to range from a minimum of $565 \mathrm{pc} / \mathrm{h} / \mathrm{ln}$ to a maximum of $1438 \mathrm{pc} / \mathrm{h} / \mathrm{ln}$ with a mean traffic volume equal to $932 \mathrm{pc} / \mathrm{h} / \mathrm{ln}$ and a standard deviation of $250.32 \mathrm{pc} / \mathrm{h} / \mathrm{ln}$. 
Figure 4.8 shows the distribution of the ramp equivalent hourly traffic volumes on the freeway deceleration SCLs. For limited length type SCLs, the equivalent traffic volume at the exit ramp is equal to the traffic volume of the vehicles that diverged from the freeway RL onto the relevant SCL. On the other hand, for extended length type SCLs, the equivalent traffic volume at the exit ramp is equal to the traffic volume of vehicles diverged from the freeway RL, vehicles already occupied the relevant SCL, and vehicles exited the freeway via the left-hand lane for two-lane exit ramp sites only. The ramp traffic volumes for all study sites ranged from a minimum of $199 \mathrm{pc} / \mathrm{h} / \mathrm{ln}$ to a maximum of $1448 \mathrm{pc} / \mathrm{h} / \mathrm{ln}$ with a mean volume of $589 \mathrm{pc} / \mathrm{h} / \mathrm{ln}$ and a standard deviation of 411.56 $\mathrm{pc} / \mathrm{h} / \mathrm{ln}$.

The following three figures (Figure 4.9, Figure 4.10, and Figure 4.11) show the distribution of vehicle classifications (percentages of $\mathrm{PC}$ and HV) on right lane upstream the freeway diverge area (RL-upstream), on right lane downstream the freeway diverge area (RL-gore), on SCL downstream the freeway diverge area at the physical exit gore (SCL-ramp), respectively. The percentages of heavy vehicles (trucks, buses, and RVs) on the RL-upstream ranged from 2.2 to $17.1 \%$ with a mean of $10.2 \%$. The percentages of HVs on the RL-gore had a mean of $12.1 \%$ ranging between 1.6 and $23.0 \%$. Whereas the $\mathrm{RL}$ average $\mathrm{HV}$ percentages along selected freeway diverge areas ranged from 1.9 to 20.1 $\%$ with a mean value of $11.2 \%$. Moreover, diverging HV percentages ranged from 1.1 to $11.0 \%$ with a mean of $4.6 \%$. Finally, the HV percentages on the SCL-ramp ranged between 1.1 and $11.0 \%$ with a mean of $5.2 \%$. 


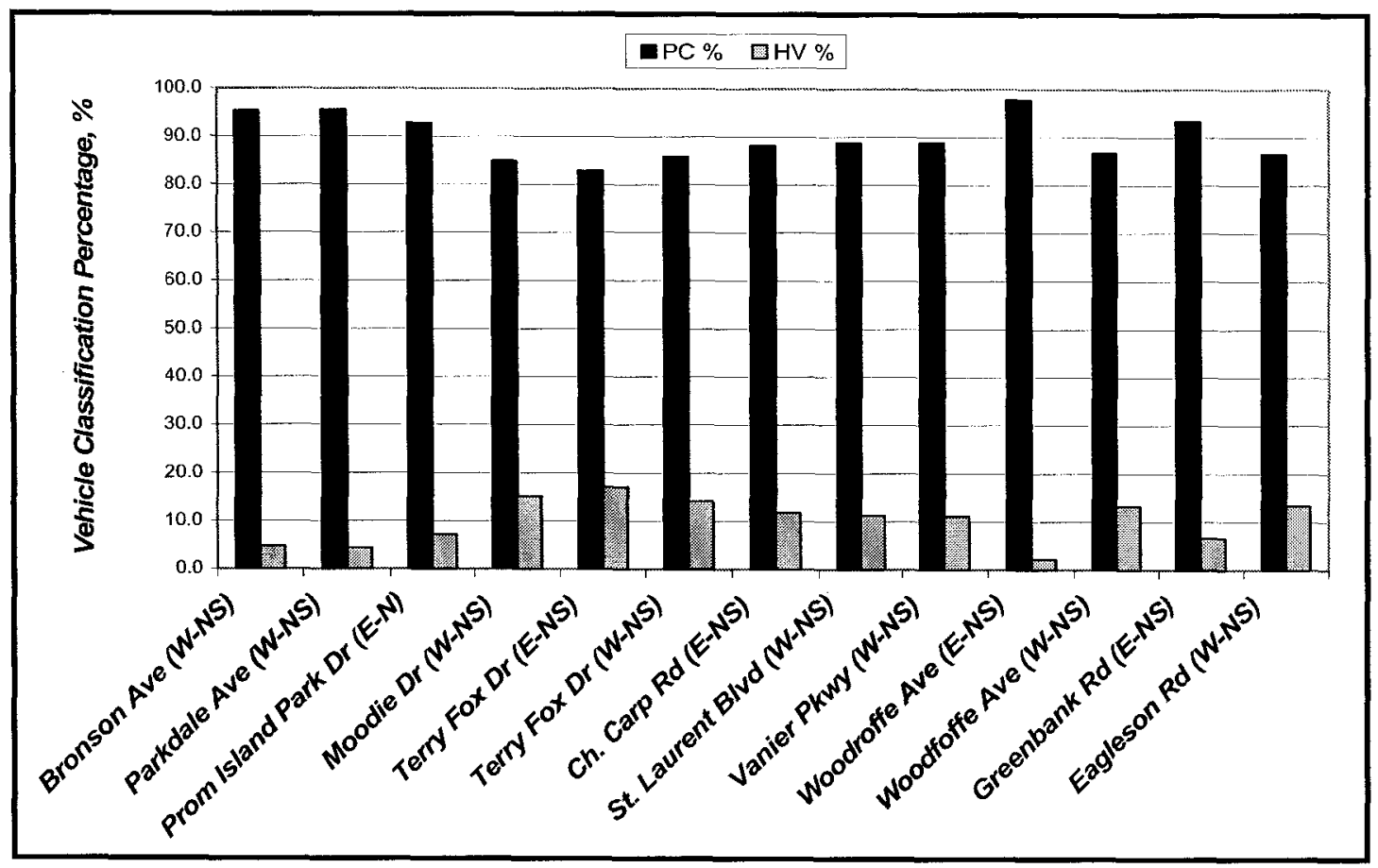

Figure 4.9: Distribution of Vehicle Classification Percentages on RL-Upstream

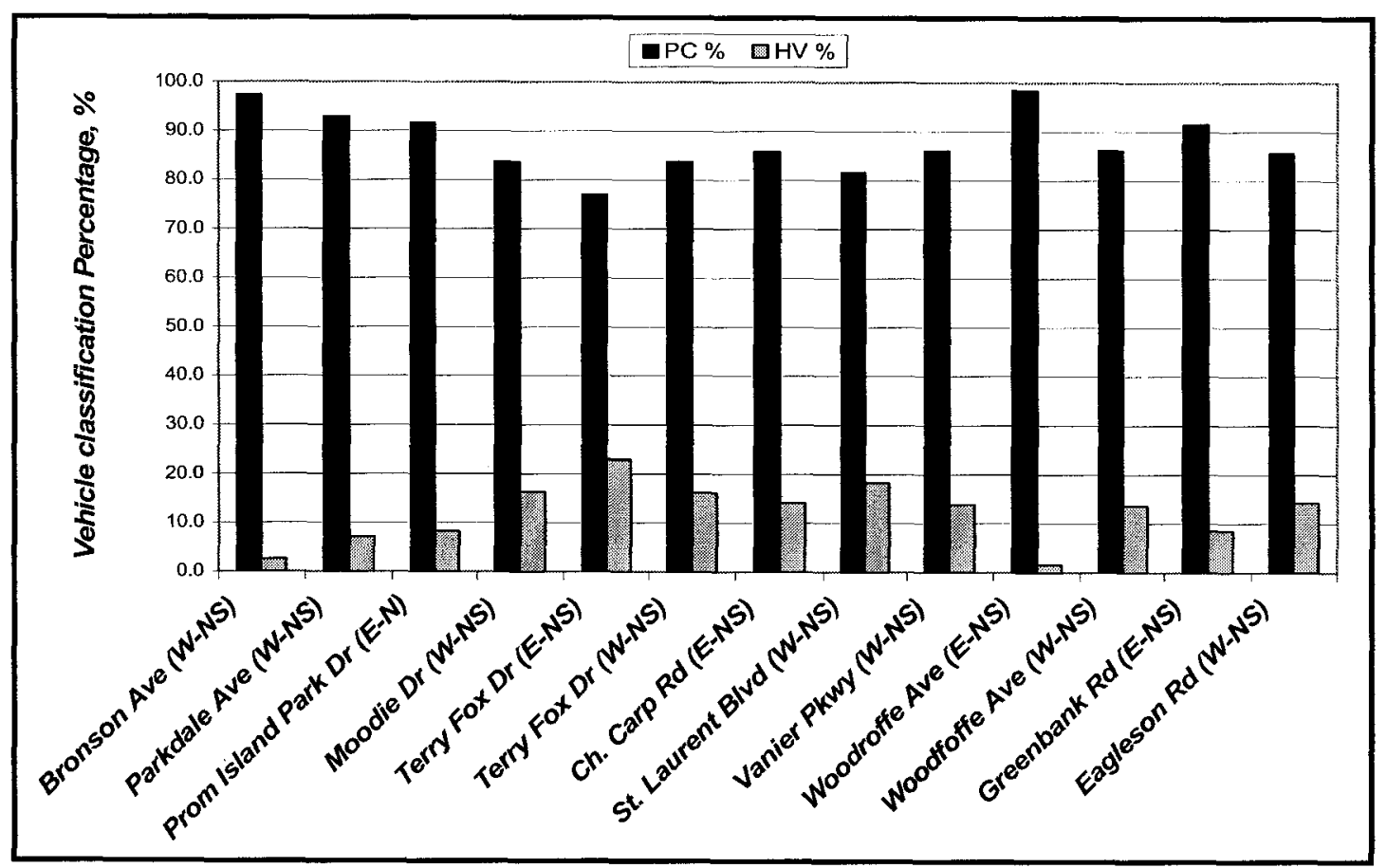

Figure 4.10: Distribution of Vehicle Classification Percentages on RL-Gore 


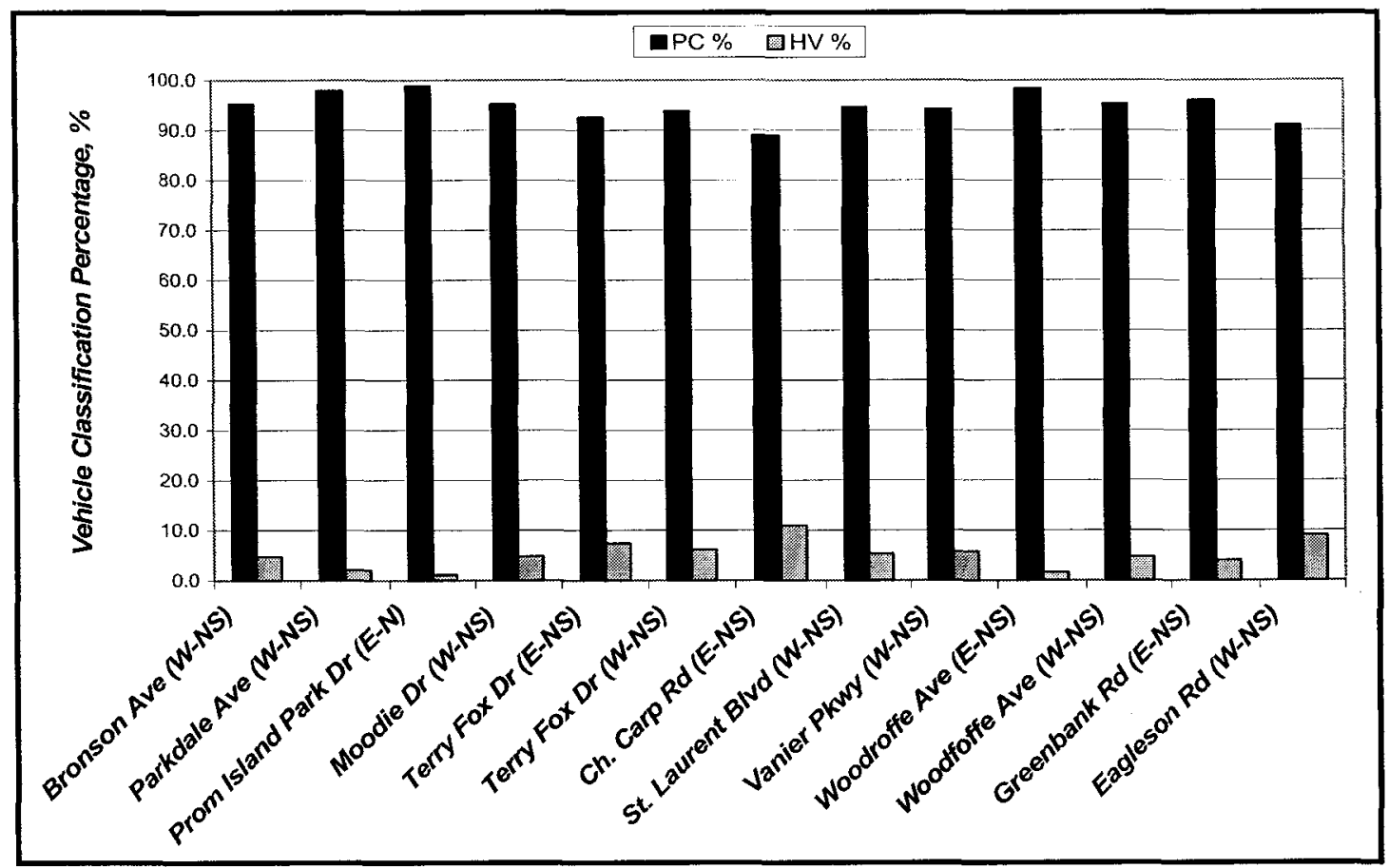

Figure 4.11: Distribution of Vehicle Classification Percentages on SCL (Ramp)

\subsubsection{Average Annual Daily Traffic (AADT)}

As mentioned earlier, the traffic volumes on the freeway mainline and ramps of Highway 417, collected by Sarhan (2004) from the MTO traffic volume database for years 2000 and 2002, were used to extract both the AADT and the peak-hour traffic volumes for the purpose of safety performance analysis. Hence, the off-peak equivalent hourly traffic volumes, collected during the year 2004 (data collection period), were compared to the peak-hour volumes extracted from the database used by Sarhan (2004) on freeway mainline and exit-ramp terminals. Figure 4.12 and Figure 4.13 demonstrate such a comparison in the form of frequency distribution charts on both freeway right lanes (mainline) and deceleration SCLs (exit ramps), respectively. It should be noted that 
the average of equivalent hourly traffic volumes on freeway right lanes were those used in comparison with the peak-hour volumes and are shown in Figure 4.12.

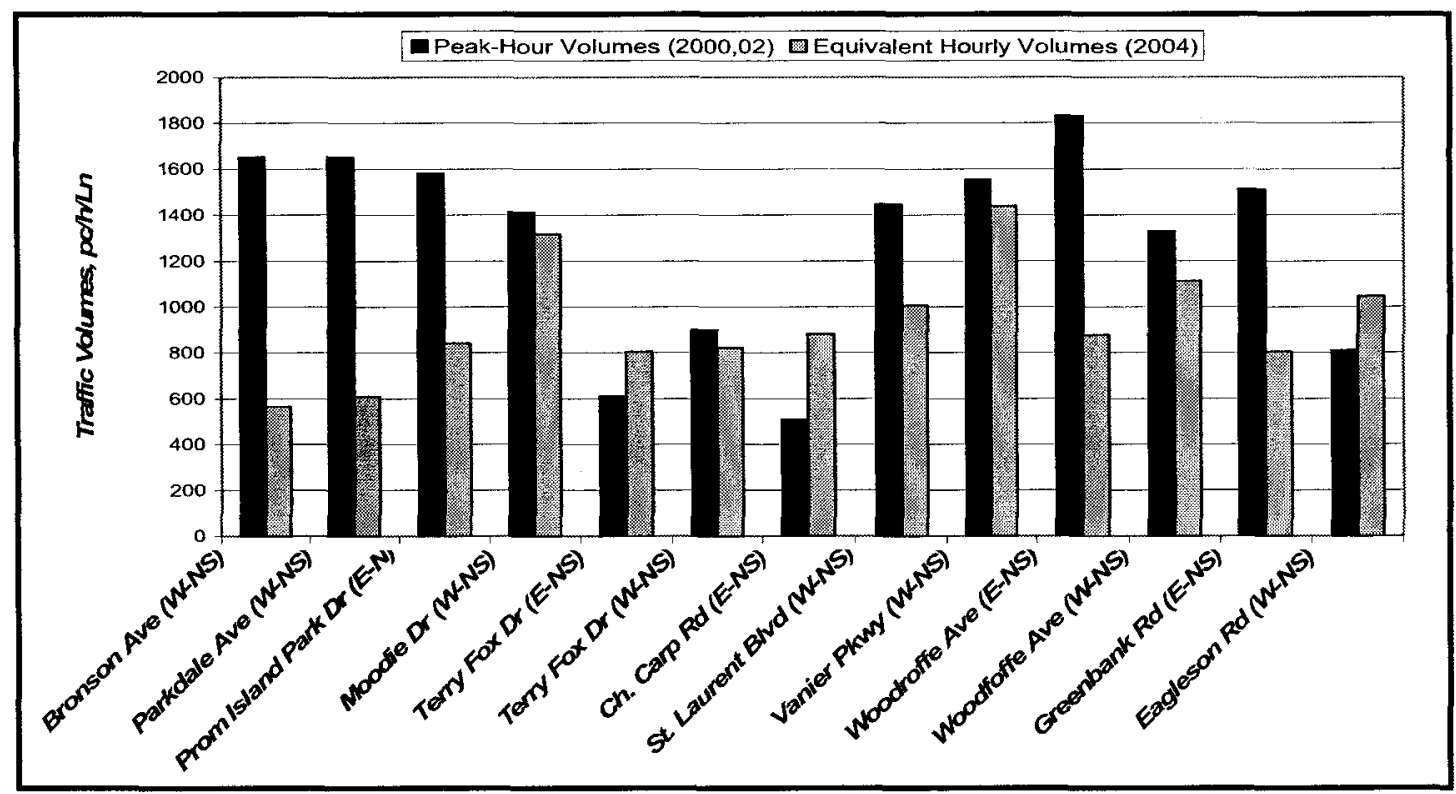

Figure 4.12: Comparison of Peak and Off-Peak Hour Traffic Volumes on Mainline

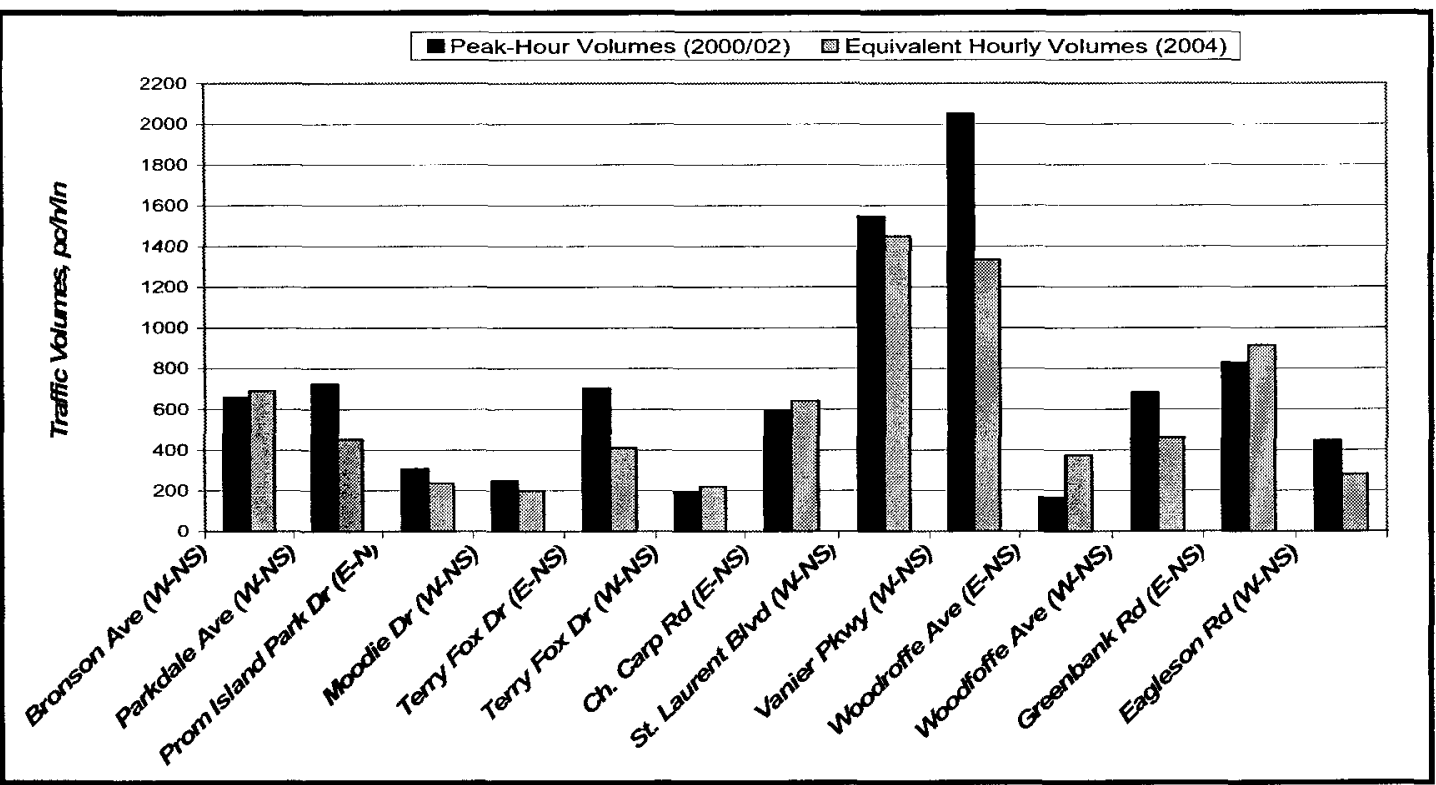

Figure 4.13: Comparison of Peak and Off-Peak Hour Traffic Volumes on Ramp 
In Figure 4.12, it is obvious that the mainline off-peak volumes are lower than the peak-hour volumes at all sites except for three sites - Terry Fox Drive E-NS, Carp Road E-NS, and Eagleson Road W-NS. As previously mentioned, the construction of the new interchange at the Castlefrank Road between Eagleson Road and Terry Fox Drive interchanges, in addition to the population growth in the western area together most likely have resulted in the redistribution of traffic conditions in this area. On the other hand, the sites in Figure 4.13 where the ramp off-peak volumes are higher than the peak-hour volumes indicate a traffic growth rates on certain exit terminals of Highway 417.

\subsection{COLLISION DATA}

As mentioned Chapter 3.0, the collision data for a five-year period (1998-2002), collected from the City of Ottawa and used by Sarhan (2004) for developing safety performance models on freeway segments, were used to extract collision data corresponding to the sites selected in this study. Afterwards, two safety parameters; collisions on freeway through segment in addition to the following deceleration SCL and the collision on the freeway deceleration SCL only $\left(\mathrm{Col}_{T h D e c}\right.$ and $\left.\mathrm{Col}_{D e c}\right)$, were prepared for analysis of safety performance on freeway diverge areas. Figure 4.14 shows the collision distributions for these two different classes. In Figure 4.14, the frequency distribution of total collisions on freeway through segments in addition to the following deceleration SCL $\left(\mathrm{Col}_{\text {ThDec }}\right)$ ranged from 7 to 110 with an average of 52 collisions and a standard deviation value of 31.8 . On the other hand, the collision frequencies on freeway deceleration SCLs only $\left(\mathrm{Col}_{D e c}\right)$ ranged from zero collisions at St. Laurent (W-NS) to 28 collisions at Vanier (W-NS) with a mean of 9 collisions and a standard deviation of 7.6. 


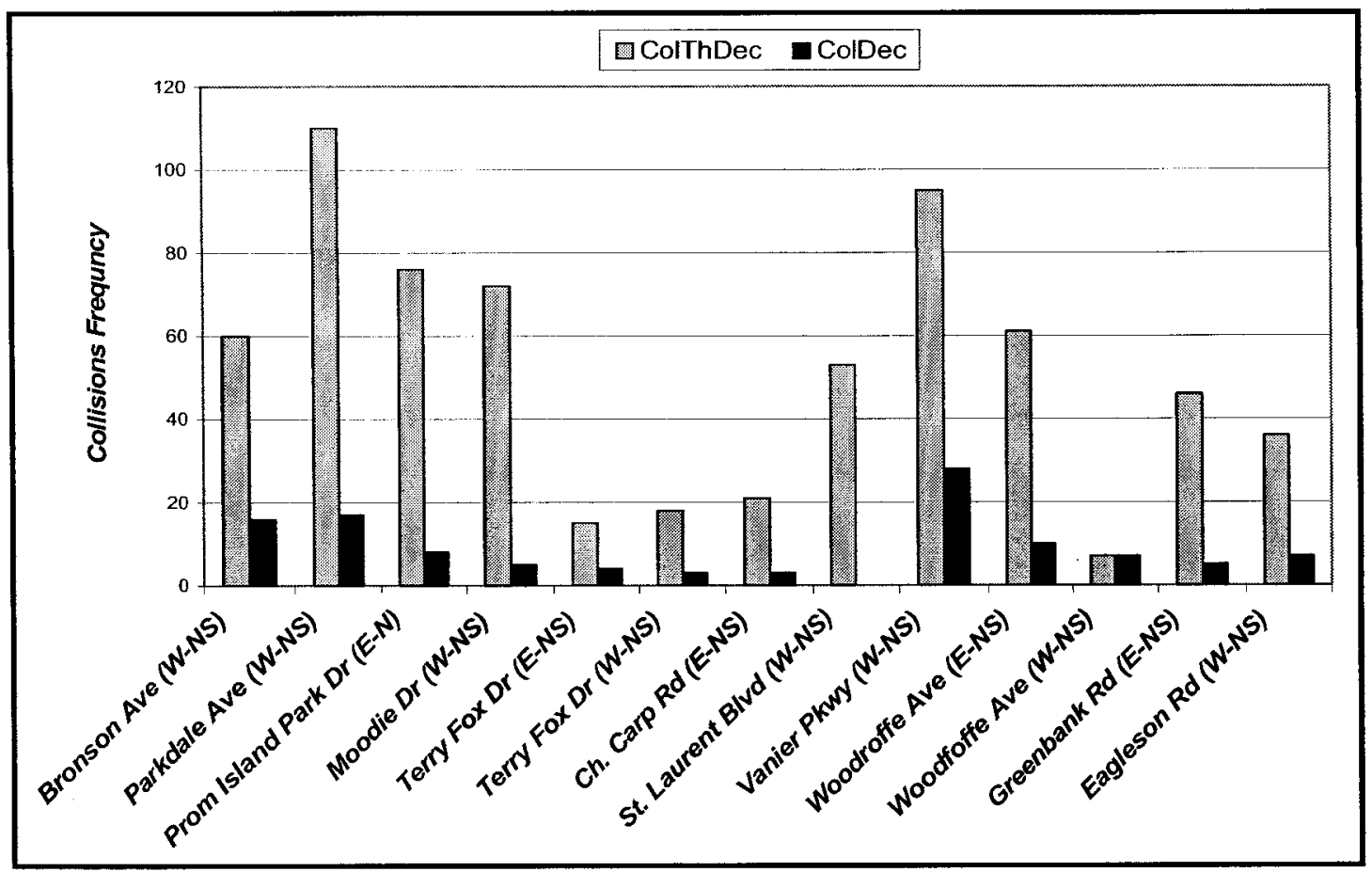

Figure 4.14: Frequency Distribution of Collisions

\subsection{AGGREGATED DRIVER BEHAVIOUR MEASURES}

\subsubsection{Distribution Tests}

The speed data collected, prepared, and described earlier consisted of several groups and sub-groups (i.e. observations with different sample sizes). Hence, distribution tests were applied to test for the distribution of the various groups of the collected data and to examine whether the groups confirm the hypothesized population distribution from which the samples were driven. For the purpose of this study, tests for skewness and the kurtosis were both used for normality testing of all the prepared data samples. By definition, skewness is a measure of symmetry of the distribution of a data set. A normally distributed sample has a skewness value of zero. Therefore, any symmetrical data sample is expected to have a skew value close to zero. On the other hand, kurtosis is 
a measure of how flat or peaked a data sample is, relative to the normal distribution pattern. Therefore, a standardized normally distributed sample is expected to have a kurtosis value equal to zero.

Accordingly, the skewness and kurtosis tests for evaluating normality were used with the assumption that any data sample that has a skewness or kurtosis value larger than \pm 1 is probably not normally distributed. It was found that all the speed, deceleration rates, and effective deceleration distance (on the freeway deceleration SCLs) as well as the mean speed (on the freeway adjacent RLs) samples, even those $<30$ and $>10$ observations, were normally distributed and had a skewness and kurtosis values within the ranges for normality assumptions. Only the samples with observations $<10 \mathrm{did}$ not satisfy the normality assumption.

\subsubsection{Test for Detecting Outliers}

An outlier is a case in which its value is different from the data values of the majority of cases in a data sample. It is important to determine and remove outliers, as they are most likely to affect the behaviour of the data sample and so change the result of the data analysis. A commonly accepted technique of determining outlier(s) is to calculate the standardized z-scores of all the data sample points (cases) and to check whether they lie between the acceptable ranges. For the purpose of this study, any data point (case) within a data sample of 30 observations or more that has a standard z-score greater than \pm 3.0 is considered an outlier and is excluded from the data sample. However, for samples with observations less than 30 and greater than 10 , data points that have a standard z-score greater than an absolute value of 2.5 were identified as outliers 
(Walpole, 2002). The standard $z$-score of a data point sample is equal to the data point value minus the data sample mean and then divided by the data sample standard deviation. . It should be noted that for each time an outlier was detected and deleted from a sample; both the sample mean and sample standard deviation were recalculated. Additionally, the total number of outliers identified for all the data samples prepared and used in this study was less than $2 \%$.

\subsubsection{Descriptive Summary}

After detecting and removing the outliers, the cumulative frequency percentage values for all data samples were calculated to determine the $85^{\text {th }}$ percentile values for the sample and population distributions. Accordingly, the student's t-distribution and zdistribution were implemented to determine the true $85^{\text {th }}$ percentile values of the population distributions of all speed data groups in this study. The commonly used statistics for describing a data set are the minimum, mean, maximum and the standard deviation measurements. Therefore, the minimum, mean, maximum, and standard deviation as well as the $85^{\text {th }}$ percentile values for all speed data samples were determined, summarized, and tabulated. Additionally, the $85^{\text {th }}$ and $95^{\text {th }}$ percentile values for the population distribution of speeds, deceleration rates, and effective deceleration distances were calculated and summarized for all samples in this study. Table 4.3 shows a descriptive summary example for the speeds (diverge, maximum, mean, and minimum) on Bronson W-NS SCL for combined PC \& HV as well as for passenger cars only, respectively. Similarly, Table 4.4 shows a descriptive summary example for the deceleration rates (overall, maximum, and mean) and the effective deceleration distances 
used on Bronson W-NS SCL for combined PC \& HV as well as for passenger cars only, respectively.

Table 4.3: Distributive Summary Sample for Speeds on Bronson (W-NS)

\begin{tabular}{|c|l|c|c|c|c|c|c|c|c|}
\hline \multirow{2}{*}{ Statistics } & \multicolumn{9}{|c|}{ Speed (km/h) } \\
\cline { 3 - 11 } \multicolumn{2}{|c|}{} & \multicolumn{3}{|c|}{ Combined PC \& HV } & \multicolumn{4}{c|}{ PC Only } \\
\cline { 2 - 11 } & Diverge & Max. & Mean & Gore & Diverge & Max. & Mean & Gore \\
\hline \multirow{5}{*}{ Sample } & N & 138 & 90 & 90 & 88 & 124 & 85 & 85 & 84 \\
\cline { 2 - 11 } & $\mathbf{8 5}^{\text {th }}$ & 94.3 & 96.8 & 90.5 & 78.6 & 94.7 & 97.3 & 90.7 & 78.7 \\
\cline { 2 - 11 } & Min. & 65.1 & 69.9 & 62.0 & 52.6 & 65.1 & 69.9 & 62.0 & 52.6 \\
\cline { 2 - 11 } & Max. & 110.0 & 109.8 & 100.6 & 87.8 & 110.0 & 109.8 & 100.6 & 87.8 \\
\cline { 2 - 11 } & Mean & 86.9 & 88.0 & 81.3 & 70.5 & 87.8 & 88.3 & 81.6 & 70.9 \\
\cline { 2 - 11 } & SD & 8.54 & 8.28 & 8.49 & 7.69 & 8.14 & 8.29 & 8.40 & 7.51 \\
\hline \multirow{3}{*}{ Population } & $\mathbf{8 5}^{\text {th }}$ & 95.7 & 96.6 & 90.1 & 78.4 & 96.6 & 96.9 & 90.3 & 78.7 \\
\cline { 2 - 11 } & $\mathbf{9 5}^{\text {th }}$ & 100.9 & 101.7 & 95.3 & 83.1 & 101.8 & 101.9 & 95.5 & 83.2 \\
\hline
\end{tabular}

Table 4.4: Distributive Summary Sample for Deceleration Rates and Effective Deceleration Distances on Bronson (W-NS)

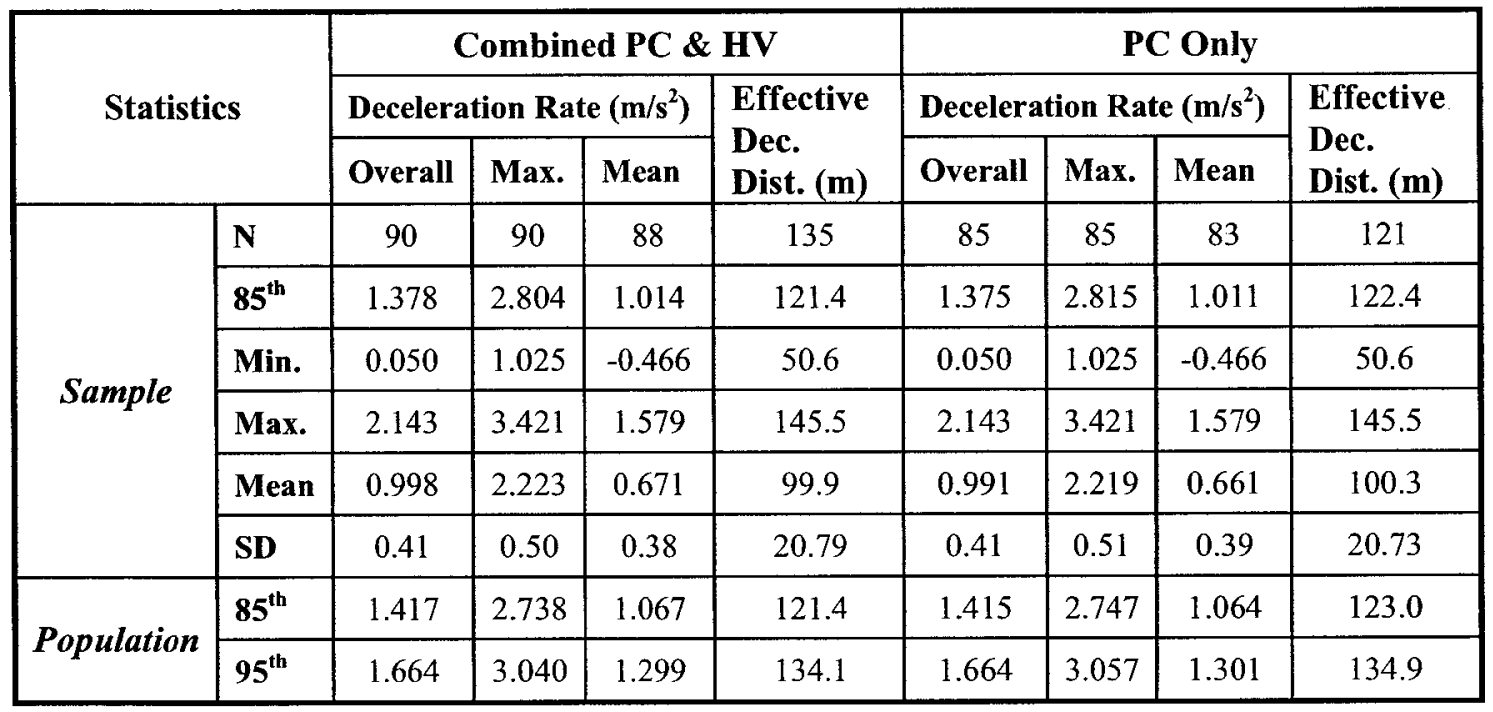


Tables summarizing the sample size, sample $85^{\text {th }}$ percentile, minimum, maximum, mean, standard deviation, and the population $85^{\text {th }}$ and $95^{\text {th }}$ percentile values for all speed data groups were prepared for further analysis and are available in Appendix C. The sample and the population $85^{\text {th }}$ percentile values for all the aggregated driver behaviour measures (analysis parameters) showed no significant discrepancies. Figure 4.15 shows an example of the sample and population $85^{\text {th }}$ percentile diverge speed on all sites selected in this study. For that reason, the population's $85^{\text {th }}$ percentile values were used for modelling procedures (presented in the next chapter).

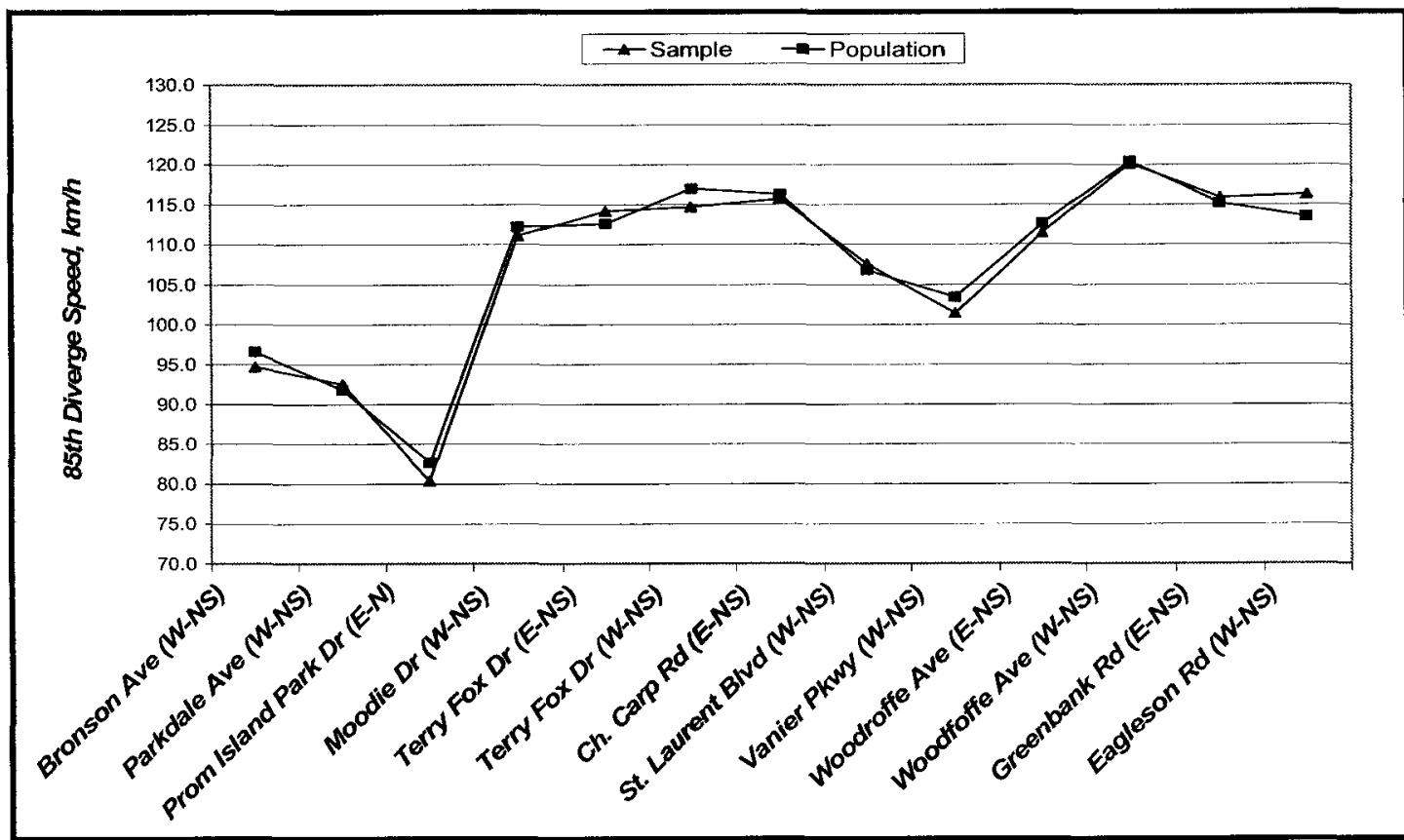

Figure 4.15: Sample and Population $85^{\text {th }}$ Percentile for Diverge-Speed

\subsubsection{Distribution of Targeted Heavy Vehicles}

The percentage of targeted heavy vehicles (HV \%) on freeway deceleration SCLs and adjacent RLs were found not to have any significant effect on the driver behaviour on 
freeway diverge areas. The two-tailed t-tests proved that differences between combined PC \& HV and passenger cars only, associated with HV percentage for all variables, were not statistically significant at $5 \%$ level of significance (all p-values $<0.05$ ). The following are five graphical presentations for the most vital variables considered in this study; namely: $R L$ mean speed (all vehicle movement), diverge speed, gore speed, overall deceleration rate, and effective deceleration distance, to illustrate the nondifference between the $85^{\text {th }}$ percentile values for combined PC \& HV and PC only.

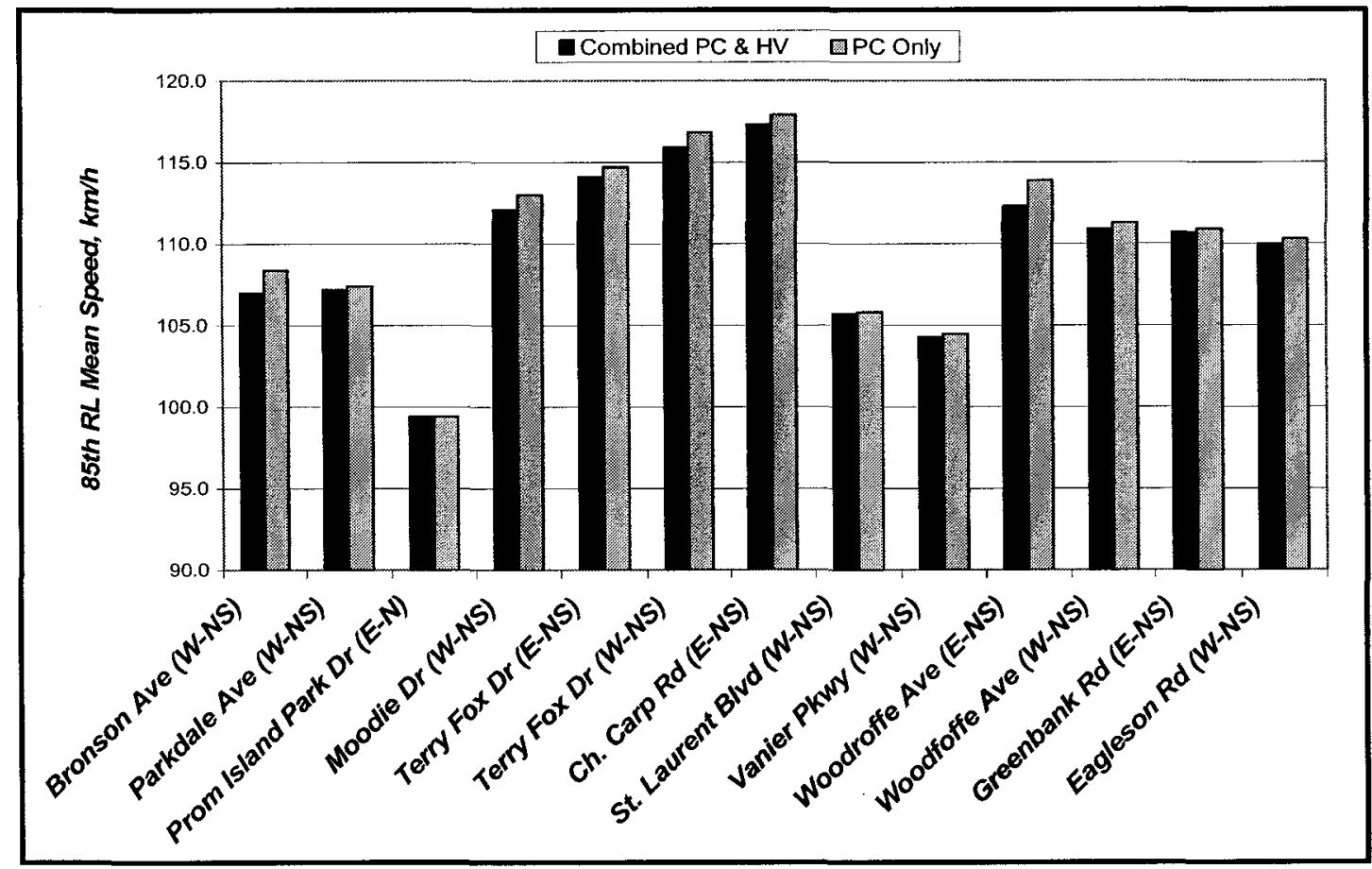

Figure 4.16: Combined PC \& HV vs. PC Only for $85^{\text {th }}$ RL Mean Speed 


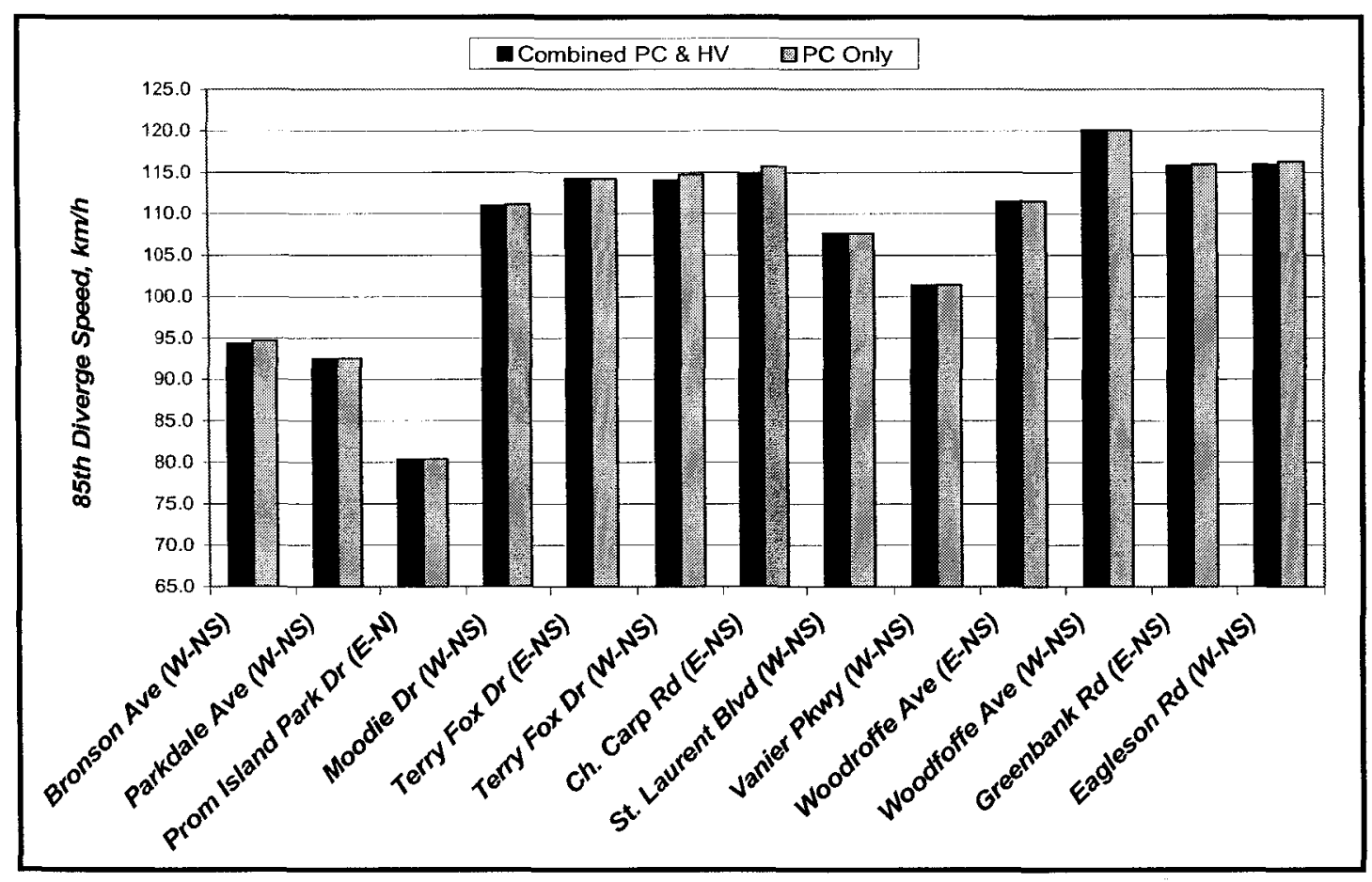

Figure 4.17: Combined PC \& HV vs. PC Only for $85^{\text {th }}$ Diverge Speed

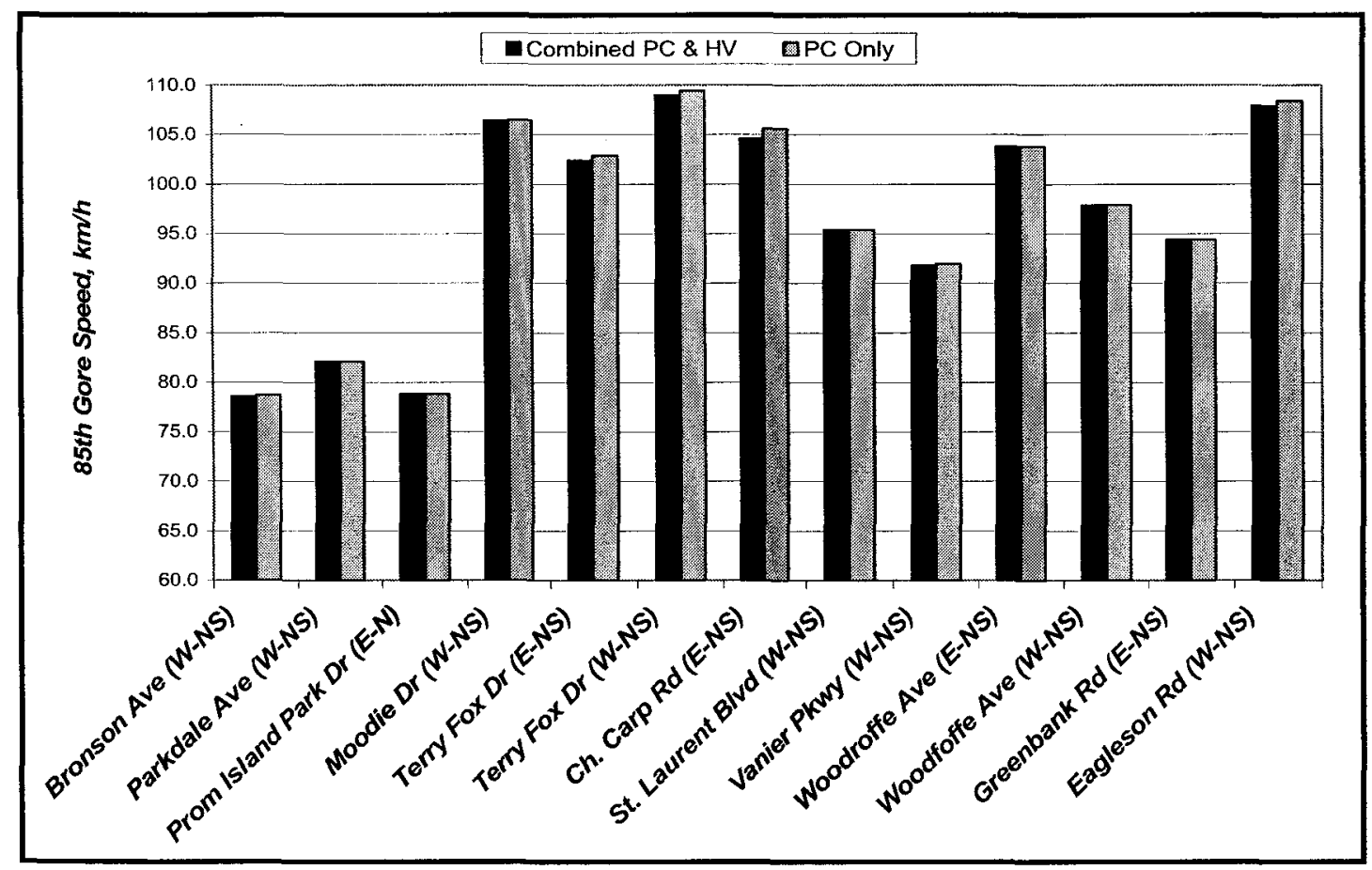

Figure 4.18: Combined PC \& HV vs. PC Only for $85^{\text {th }}$ Gore Speed 


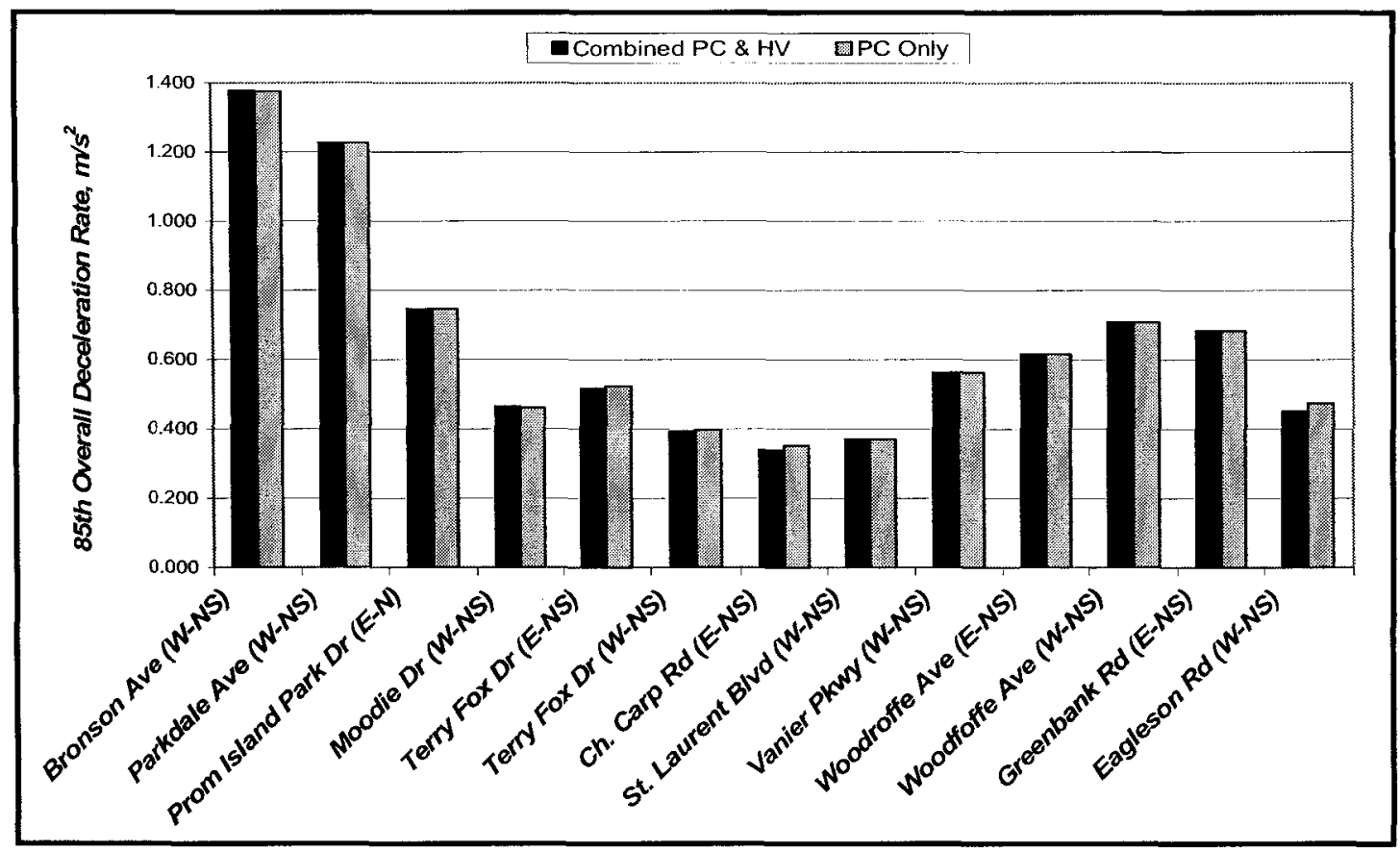

Figure 4.19: Combined PC \& HV vs. PC Only for $85^{\text {th }}$ Overall Deceleration Rate

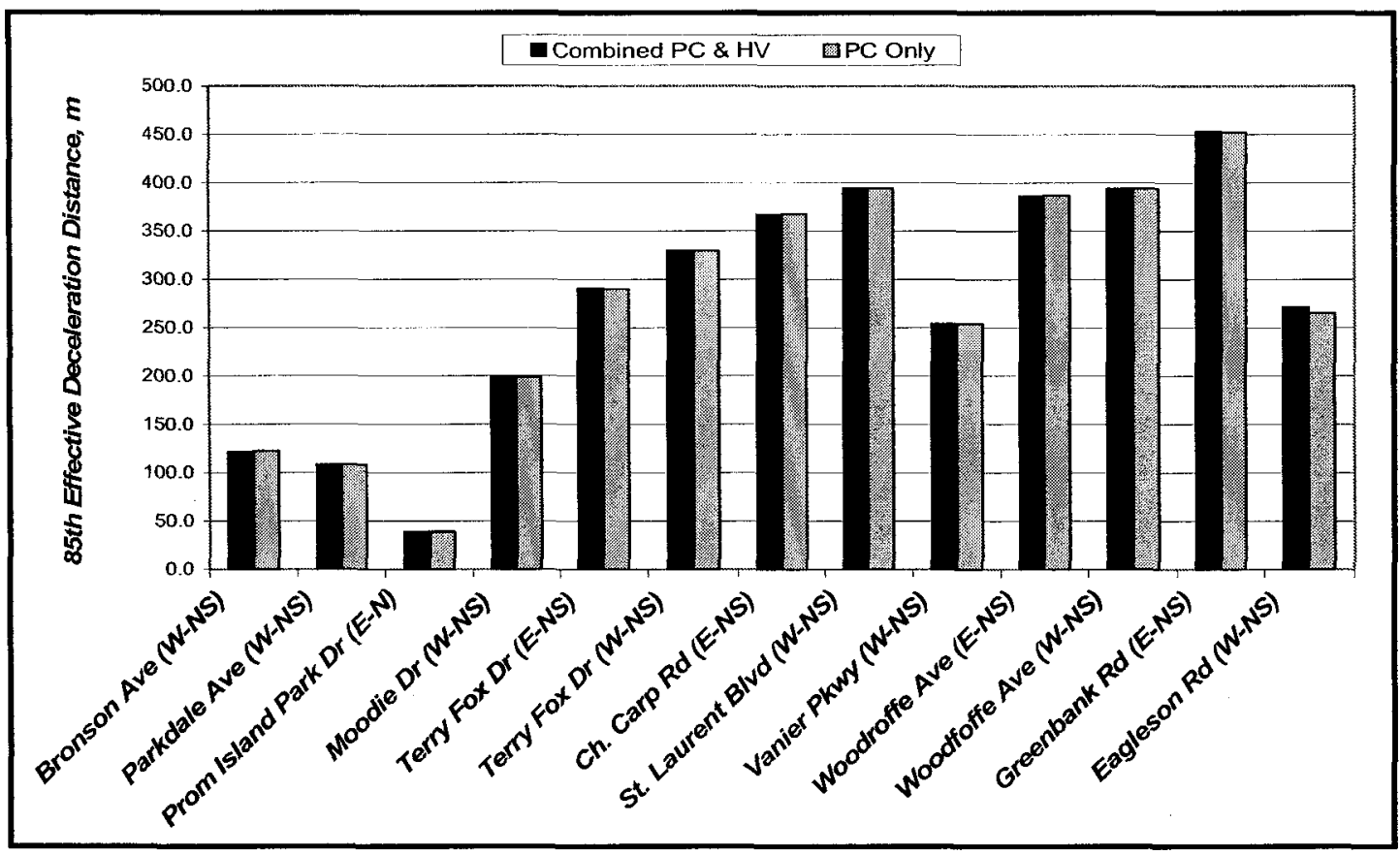

Figure 4.20: Combined PC \& HV vs. PC Only for $85^{\text {th }}$ Effective Deceleration Distance 


\subsubsection{Distribution of RL Mean Speeds}

The speed data collected on freeway RLs adjacent to deceleration SCLs on the selected study sites were categorized into two main categories of vehicles on freeway RL namely: "all vehicle movements" and "through vehicles only". All vehicle movements refer to vehicles recorded upstream the freeway diverge area including those diverging onto the SCL and/or merging left onto freeway inner lane. On the other hand, through vehicles only are those continuing on the freeway $\mathrm{RL}$ and recorded to the proximity of the gore area (limits of exit ramp terminal). Figures 4.21 and 4.22 show distributions of RL mean speeds for all vehicle movements and through vehicles, respectively.

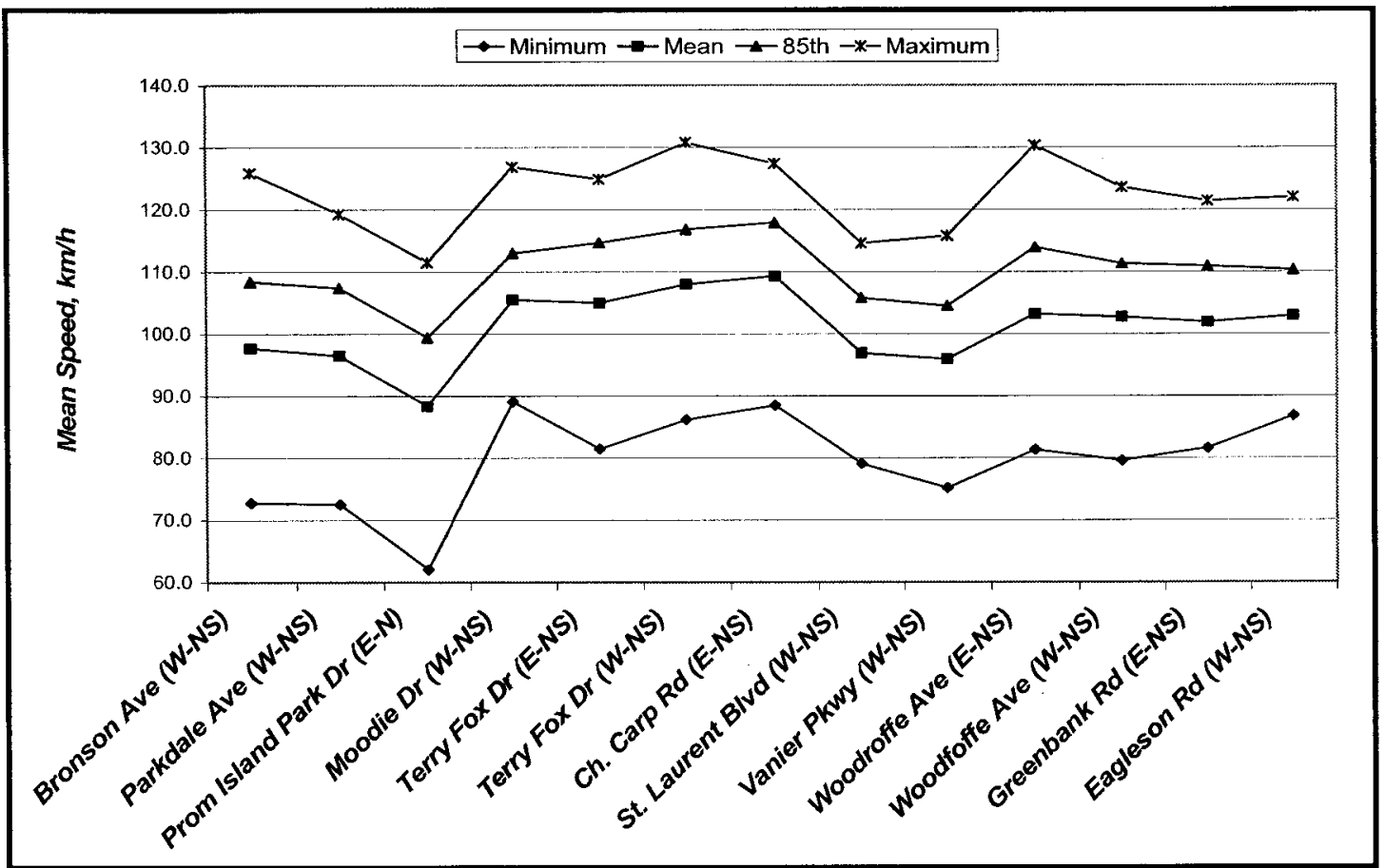

Figure 4.21: Distribution of RL Mean Speeds of All Vehicle Movements 


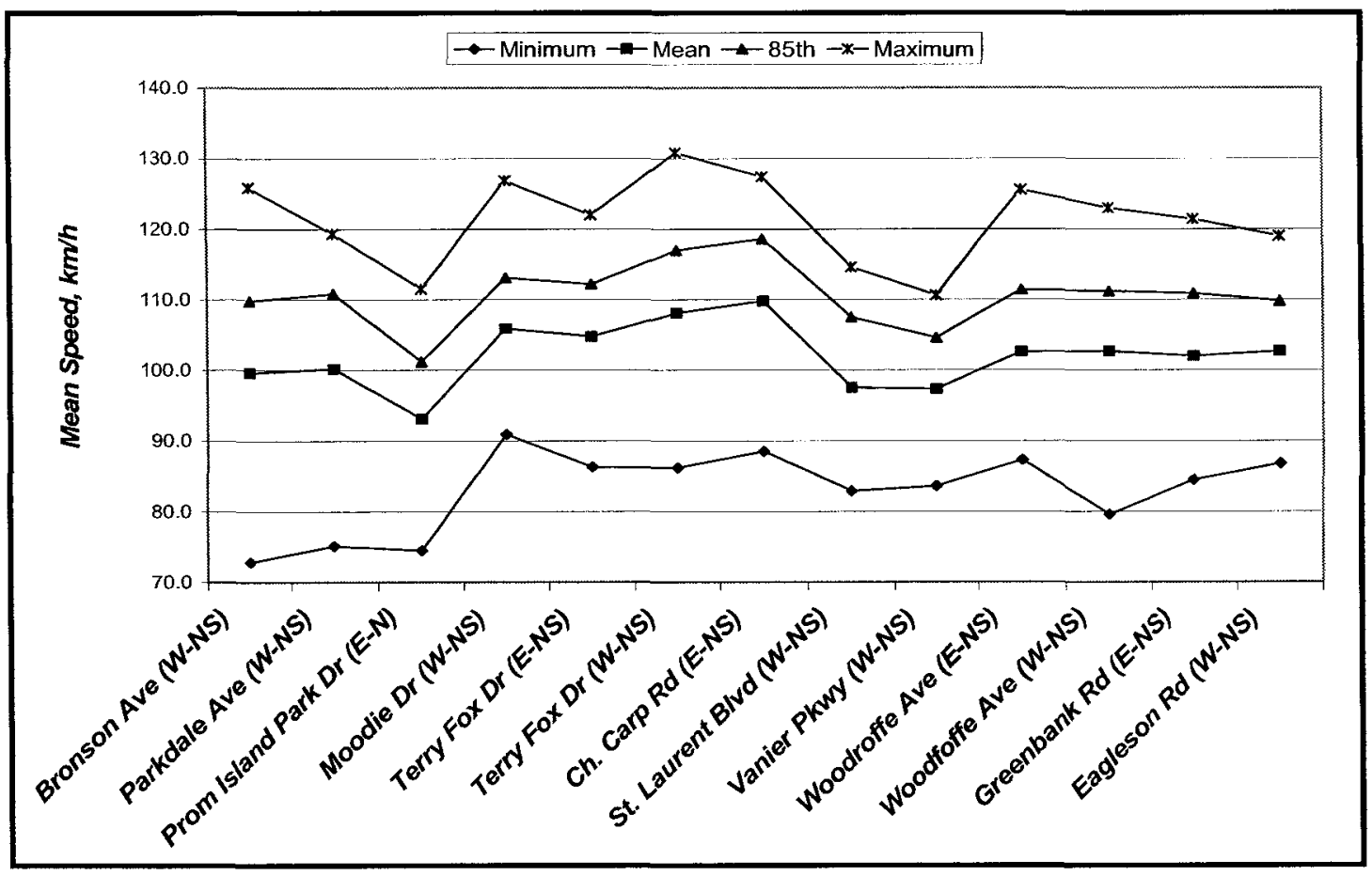

Figure 4.22: Distribution of RL Mean Speeds of Through Vehicles Only

As shown in the figures, RL mean speeds ranged from $62.1 \mathrm{~km} / \mathrm{h}$ to $130.8 \mathrm{~km} / \mathrm{h}$ and from $72.8 \mathrm{~km} / \mathrm{h}$ to $130.8 \mathrm{~km} / \mathrm{h}$ for all movements and through vehicles, respectively. However, the $85^{\text {th }}$ percentile RL mean speed ranged from $99.4 \mathrm{~km} / \mathrm{h}$ to $117.9 \mathrm{~km} / \mathrm{h}$ and from $101.2 \mathrm{~km} / \mathrm{h}$ to $118.6 \mathrm{~km} / \mathrm{h}$ for all movements and through vehicles, respectively. The data revealed that RL mean speeds for all vehicles movements generated lower speeds than through vehicles only. This is due to the presence of vehicles diverging onto SCL and/or merging left onto inner lane amongst all vehicles movements. Hence, twotailed $\mathrm{t}$-tests were performed to test for differences between RL mean speeds of all vehicle movements and through vehicles only for all sites in the study. The t-tests showed that the differences were not statistically significant at $5 \%$ level of significance (all $p$ values $<0.05$ ) for all sites. Figure 4.23 shows graphical distribution of $85^{\text {th }}$ percentile and 
mean RL mean speed on all sites for such a comparison between all vehicle movements and through vehicles only. Thus, further analysis performed in the study considered RL mean speeds for all vehicle movements to take into consideration the effect of diverging and/or merging vehicles onto the freeway SCL and/or inner lane.

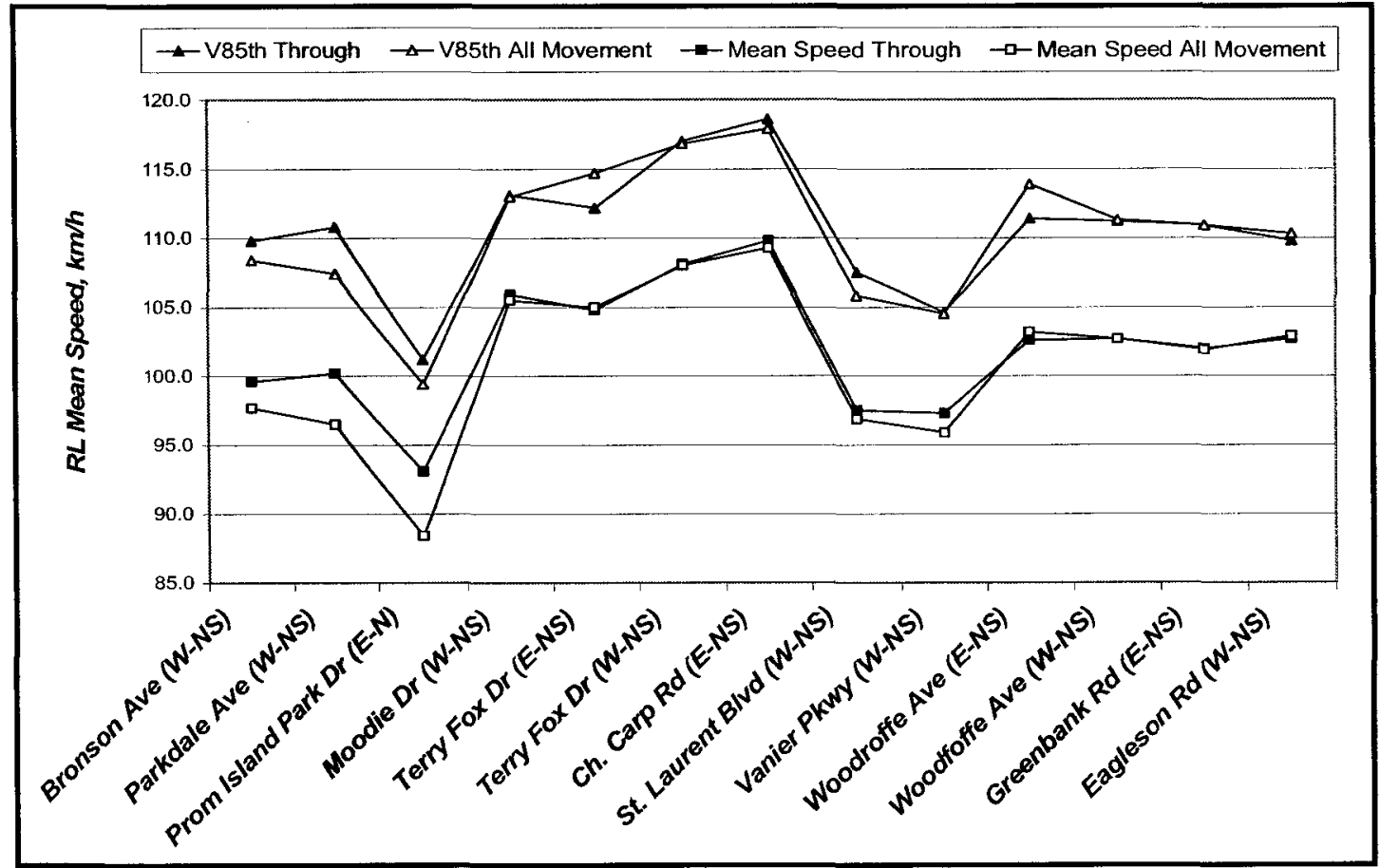

Figure 4.23: Comparison between All Movement and Through RL Mean Speeds

\subsubsection{Distribution of Speeds on SCL}

As mentioned earlier, fours measures were extracted for the collected speed data on the deceleration lanes; namely: diverge speed, gore speed, mean speed, and maximum speed. Figure 4.24 displays the $85^{\text {th }}$ percentile distribution of these four speed measures on all sites. This section provides a description of the diverge and gore speed measures and their distributions among the study sites. 


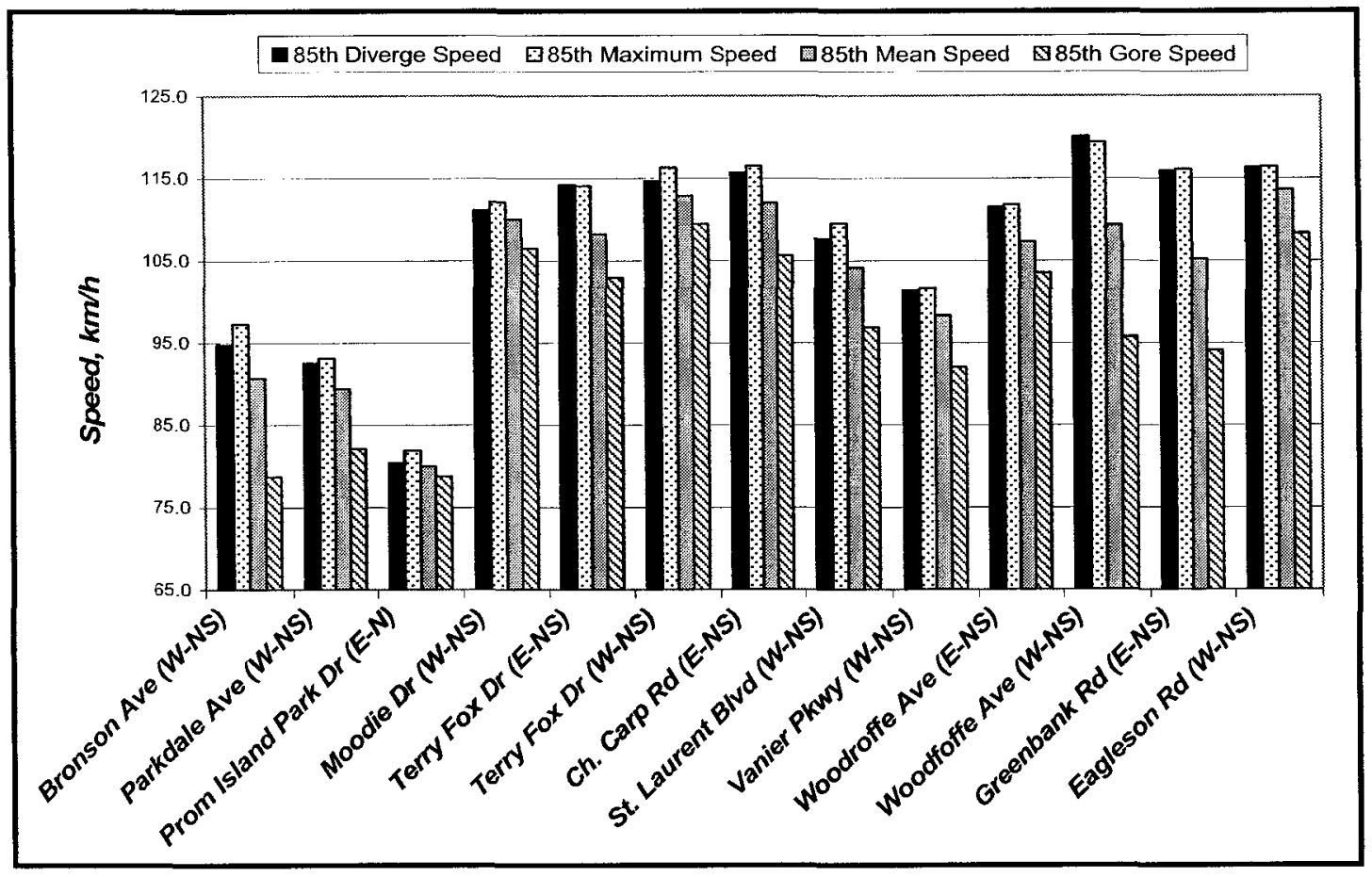

Figure 4.24: Frequency Distribution of Speeds on SCL

\subsection{6.a Diverge Speed}

Figure 4.25 shows the distribution of minimum, mean, $85^{\text {th }}$ percentile, and maximum diverge speeds on all sites. The diverge speed among all study sites ranged from a lowest diverge speed of $53.0 \mathrm{~km} / \mathrm{h}$ to a highest diverge speed of $138.0 \mathrm{~km} / \mathrm{h}$. The $85^{\text {th }}$ percentile diverge speeds ranged from $80.4 \mathrm{~km} / \mathrm{h}$ at Island Park E-N to $120.1 \mathrm{~km} / \mathrm{h}$ at Woodroffe W-NS with mean $85^{\text {th }}$ percentile diverge speed of $107.4 \mathrm{~km} / \mathrm{h}$ for all thirteen sites. It should be noted that Island Park E-N is the shortest limited length type deceleration lane with a length of $58 \mathrm{~m}$, while Woodroffe W-NS is an extended length type deceleration lane with a length equal to 1358 meters. Moreover, it can be seen in Figure 4.24 that Woodroffe W-NS experienced diverge speed higher than the maximum speed. This is due to the contribution of the diverging vehicles targeted on the freeway 
right lane. Those diverging vehicles (targeted on right lane up to the diverge point) were not targeted after travelling on the deceleration SCL and therefore no maximum speed was recorded for them on the SCL.

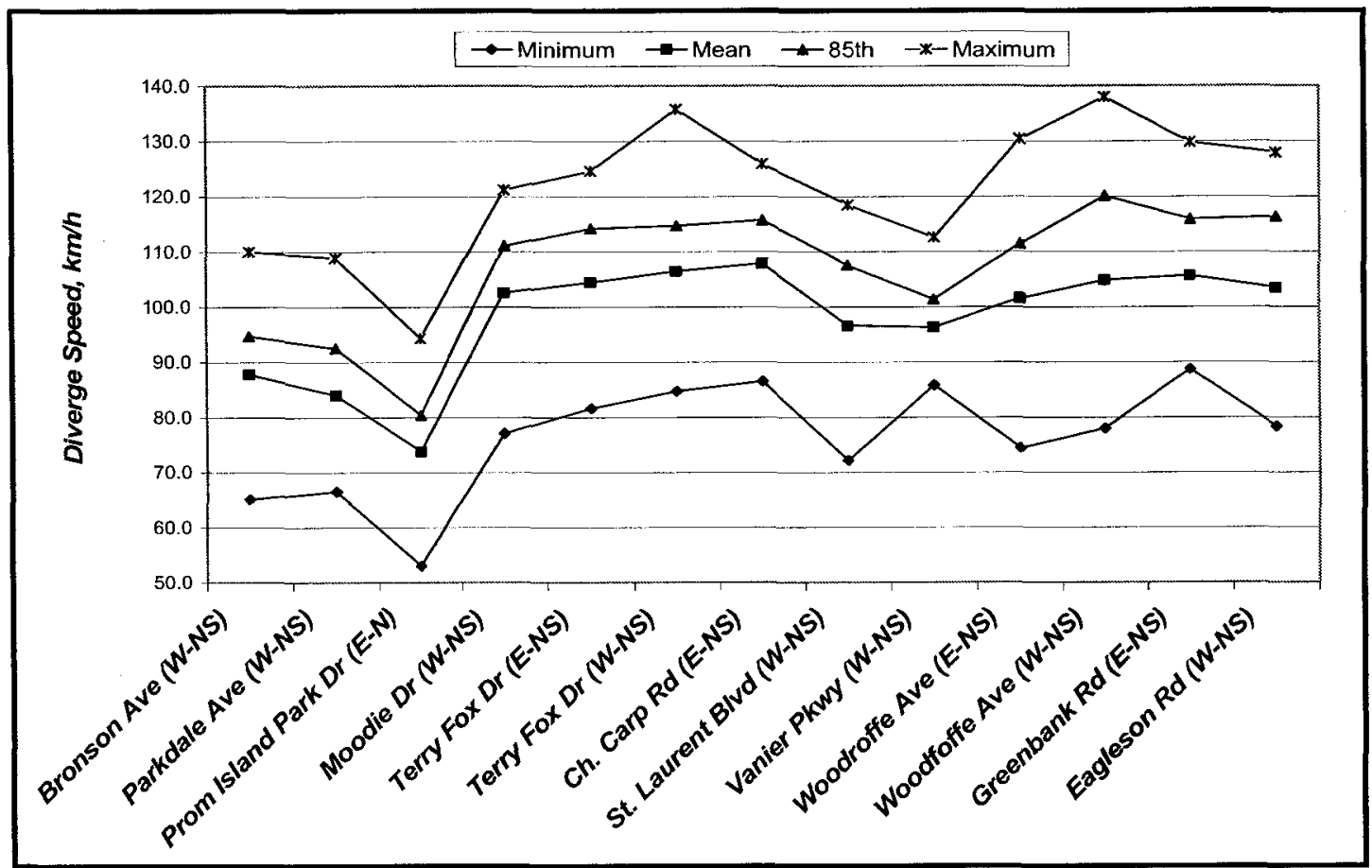

Figure 4.25: Distribution of Diverge Speed on SCL

\subsection{6.b Gore Speed}

Figure 4.26 shows the distribution of minimum, mean, $85^{\text {th }}$ percentile, and maximum gore speeds on all sites. The gore speed among all sites ranged from a lowest gore speed of $48.1 \mathrm{~km} / \mathrm{h}$ to a highest gore speed $126.6 \mathrm{~km} / \mathrm{h}$. The $85^{\text {th }}$ percentile gore speeds ranged from $78.7 \mathrm{~km} / \mathrm{h}$ at both Bronson W-NS and Island Park E-N to $109.4 \mathrm{~km} / \mathrm{h}$ at Terry Fox W-NS with mean $85^{\text {th }}$ percentile gore speed of $96.6 \mathrm{~km} / \mathrm{h}$. In Figure 4.26 , it should be noted that gore speeds for Woodroffe E-NS are not recorded at end of SCL, but 
somewhere before the end of the SCL (1.25 $\mathrm{m}$ separation), as previously explained in Sections 4.2 and 4.3. Hence, this data point was not included for further analysis.

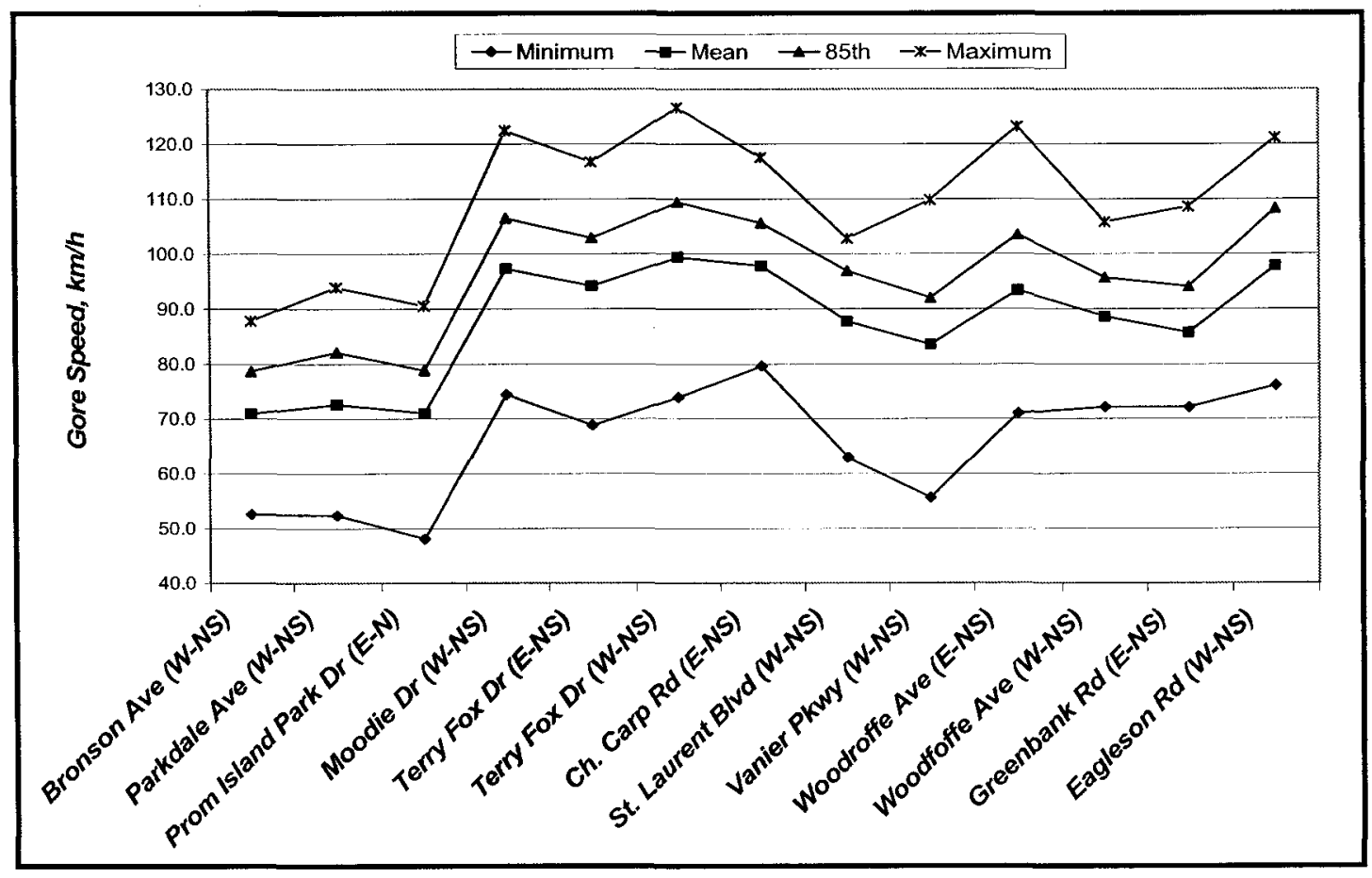

Figure 4.26: Distribution of Gore Speed on SCL

Once again, Island Park E-N was the shortest deceleration lane with a length of 58 meters among all sites and showed a gore speed of $78.8 \mathrm{~km} / \mathrm{h}$. The same $85^{\text {th }}$ gore speed was shown on Bronson W-NS that has a length of 212 meters. On the other hand, Terry Fox W-NS has the longest deceleration limited length of $446 \mathrm{~m}$ among sites and showed a percentile $85^{\text {th }}$ gore speed of $109.4 \mathrm{~km} / \mathrm{h}$ (highest among sites). 


\subsubsection{Distribution of Deceleration Rates on SCL}

As mentioned earlier, three measures were extracted for the deceleration speed profiles collected on the freeway deceleration lanes; namely: overall deceleration rate, mean deceleration rate, and maximum deceleration rate. Figure 4.27 displays the $85^{\text {th }}$ percentile distribution of these three deceleration measures on all sites. This section provides a description of the overall, mean, and maximum deceleration rate measures and their distributions among the study sites.

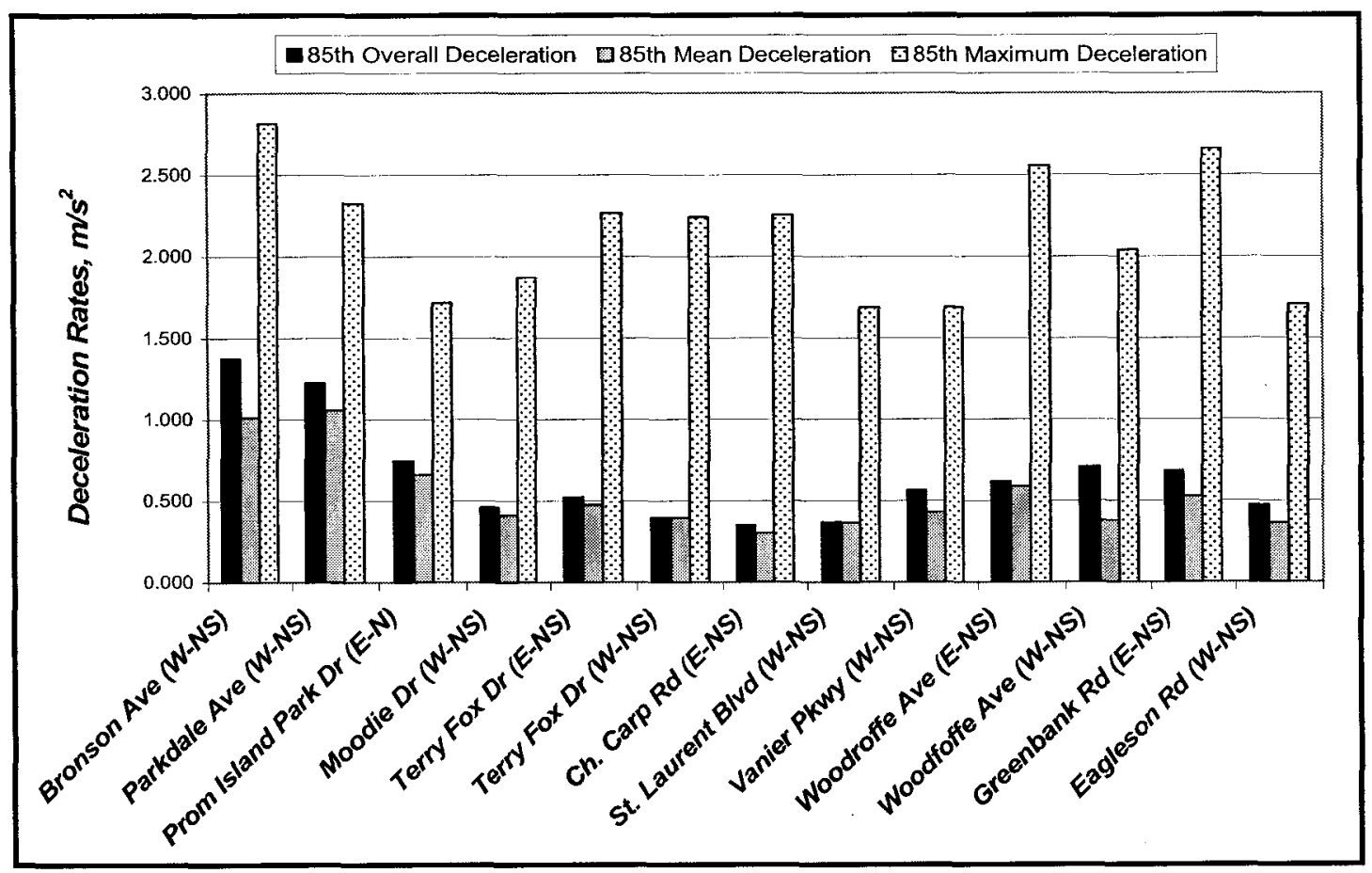

Figure 4.27: Frequency Distribution of Deceleration Rates on SCL

The $85^{\text {th }}$ overall deceleration rates among all sites ranged from $0.351 \mathrm{~m} / \mathrm{sec}^{2}$ at Carp E-NS to $1.375 \mathrm{~m} / \mathrm{sec}^{2}$ at Bronson W-NS with a mean overall deceleration rate equal to $0.654 \mathrm{~m} / \mathrm{sec}^{2}$. Similarly, the $85^{\text {th }}$ mean deceleration rates among all sites ranged from a 
minimum of $0.301 \mathrm{~m} / \mathrm{sec}^{2}$ at Carp E-NS to maximum of $1.055 \mathrm{~m} / \mathrm{sec}^{2}$ at Parkdale W-NS with a mean value of $0.535 \mathrm{~m} / \mathrm{sec}^{2}$. Finally, the $85^{\text {th }}$ maximum deceleration rates among all sites ranged from $1.688 \mathrm{~m} / \mathrm{sec}^{2}$ at both St. Laurent W-NS and Vanier W-NS to 2.815 $\mathrm{m} / \mathrm{sec}^{2}$ at Bronson W-NS with a mean rate of $2.140 \mathrm{~m} / \mathrm{sec}^{2}$.

\subsection{7.a Overall Deceleration Rate}

Figure 4.28 shows the distribution of minimum, mean, $85^{\text {th }}$ percentile, and maximum overall deceleration rates on all sites. The deceleration rate among all sites ranged from a lowest overall deceleration rate of $-0.509 \mathrm{~m} / \mathrm{sec}^{2}$ (i.e. an acceleration rate) to a highest overall deceleration rate of $2.143 \mathrm{~m} / \mathrm{sec}^{2}$. The $85^{\text {th }}$ overall deceleration rates ranged from $0.351 \mathrm{~m} / \mathrm{sec}^{2}$ at Carp E-NS to $1.375 \mathrm{~m} / \mathrm{sec}^{2}$ at Bronson W-NS. The two lowest overall deceleration rates of $0.370 \mathrm{~m} / \mathrm{sec}^{2}$ and $0.397 \mathrm{~m} / \mathrm{sec}^{2}$ were shown on Terry Fox W-NS and St. Laurent W-NS. It should be noted that St. Laurent W-NS is an extended length type SCL, while Carp E-NS and Terry Fox W-NS are two limited length types SCLs with lengths of $430 \mathrm{~m}$ and $446 \mathrm{~m}$ (longest two limited deceleration lanes), respectively. On the other hand, Bronson W-NS showed the highest overall deceleration rate of $1.375 \mathrm{~m} / \mathrm{sec}^{2}$ and it has a length of 212 meters. 


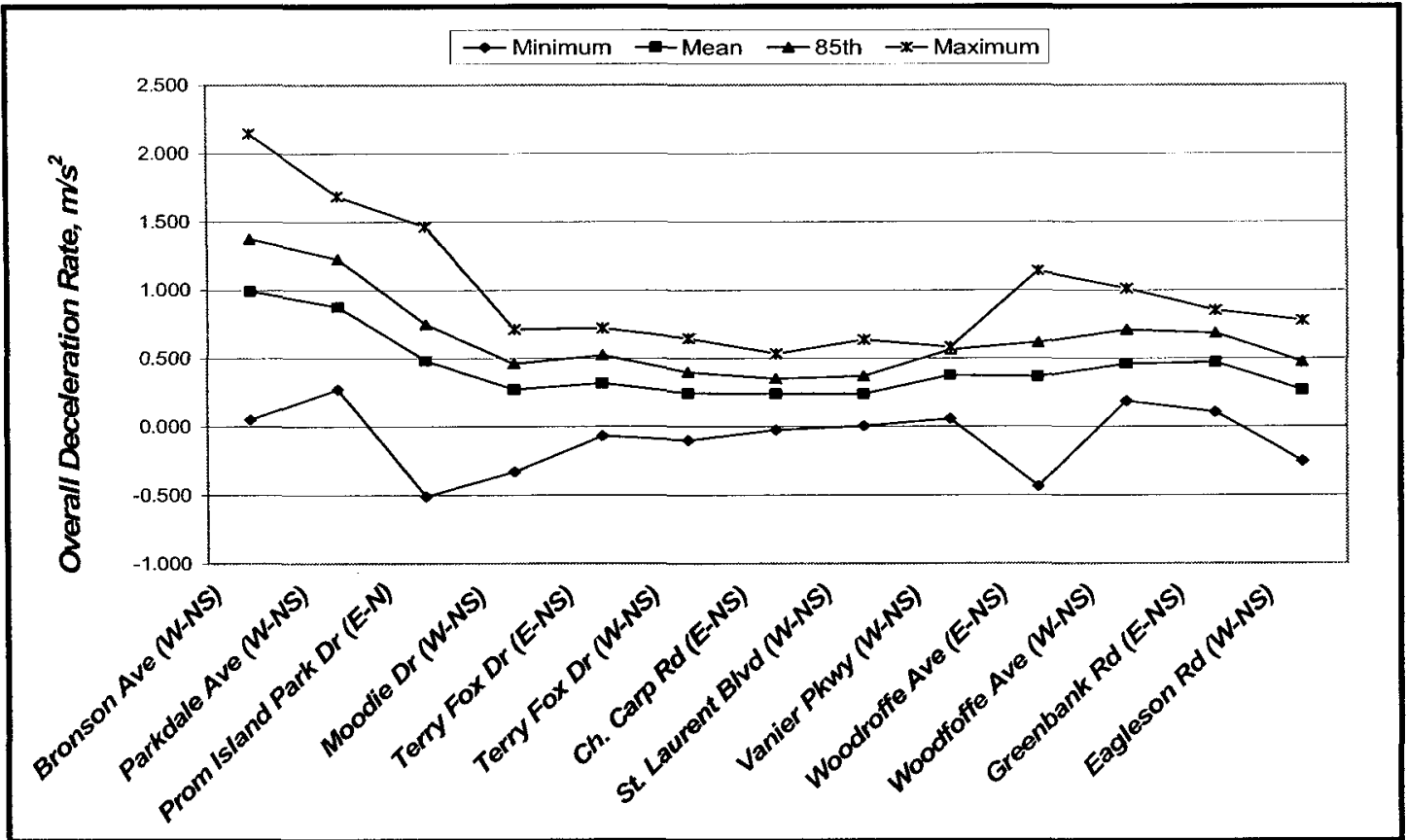

Figure 4.28: Distribution of Overall Deceleration Rate on SCL

\subsection{7.b Mean Deceleration Rate}

Figure 4.29 shows difference between the $85^{\text {th }}$ overall and $85^{\text {th }}$ mean deceleration rates on all sites under study. Graphically, the differences between the overall and mean deceleration rates were evident at some sites. Hence, two-tailed t-tests were conducted to test for the difference between these two deceleration rates among all sites. The t-tests proved that the differences between $85^{\text {th }}$ overall and $85^{\text {th }}$ mean deceleration rates were statistically significant at $5 \%$ level of significance for Bronson W-NS, Parkdale W-NS, Vanier W-NS, and Woodroffe W-NS (all p-values $>0.05$ ), but were not statistically significant for rest of sites (all $p$-values $<0.05$ ). Nevertheless, $85^{\text {th }}$ percentile overall and mean deceleration rates were considered for further analysis. However, it should be noted that the overall deceleration rate was considered as a better indicator as it captures the whole distance and time taken for vehicle deceleration behaviour on SCL. 


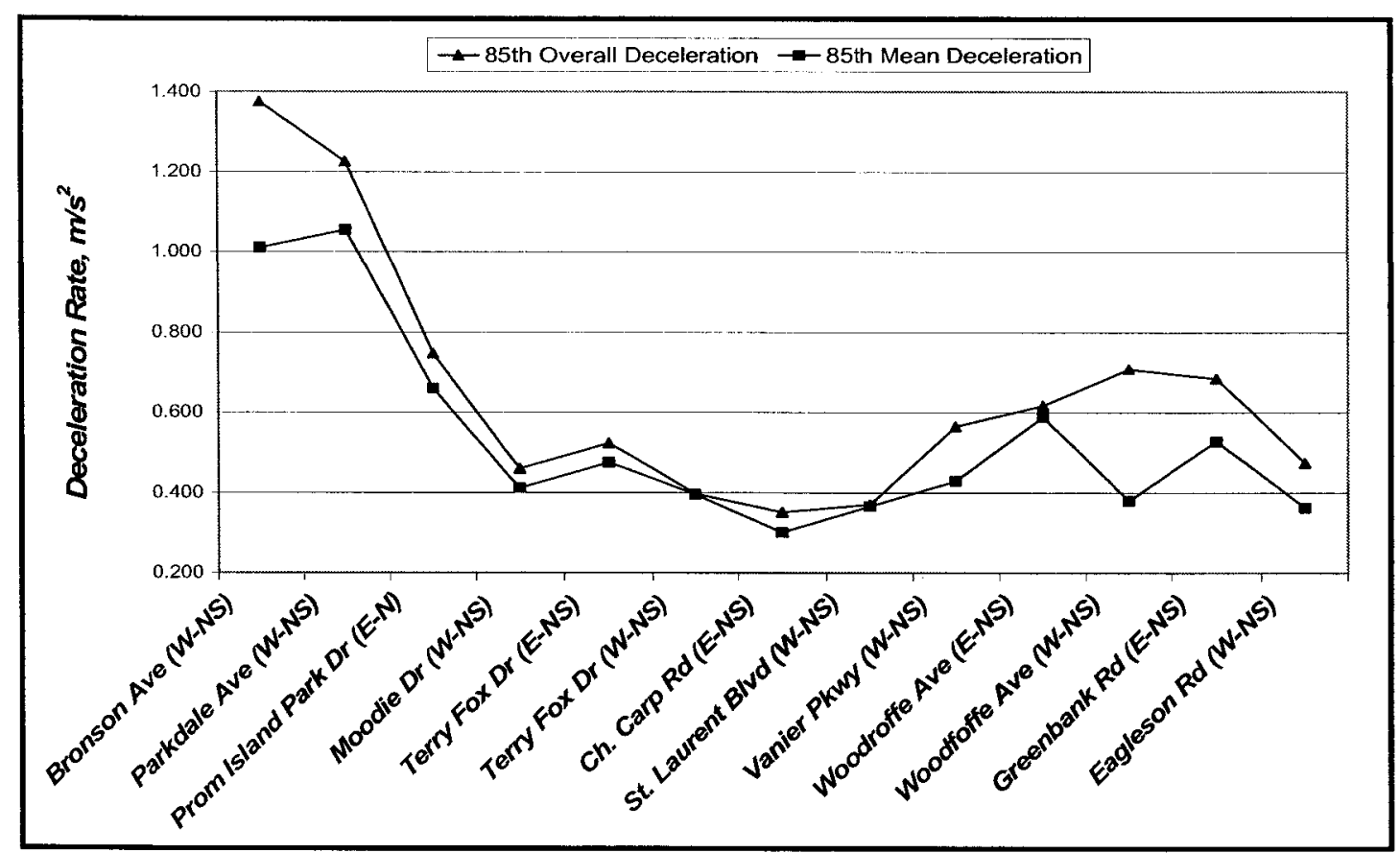

Figure 4.29: Overall and Mean $85^{\text {th }}$ Percentile Deceleration Rates

\subsection{7.c Maximum Deceleration Rate}

Figure 4.30 shows the distribution of mean and $85^{\text {th }}$ percentile maximum deceleration rates on all sites. The maximum deceleration rates among sites ranged from a lower maximum deceleration rate of $0.004 \mathrm{~m} / \mathrm{sec}^{2}$ to a higher maximum deceleration rate of $3.421 \mathrm{~m} / \mathrm{sec}^{2}$. The $85^{\text {th }}$ maximum deceleration rate among all sites in this study ranged from $1.688 \mathrm{~m} / \mathrm{sec}^{2}$ at both St. Laurent W-NS and Vanier W-NS to $2.815 \mathrm{~m} / \mathrm{sec}^{2}$ on Bronson W-NS with a mean maximum deceleration rate of $2.140 \mathrm{~m} / \mathrm{sec}^{2}$. This $85^{\text {th }}$ mean maximum deceleration rate can be used as the maximum comfortable deceleration rate. 


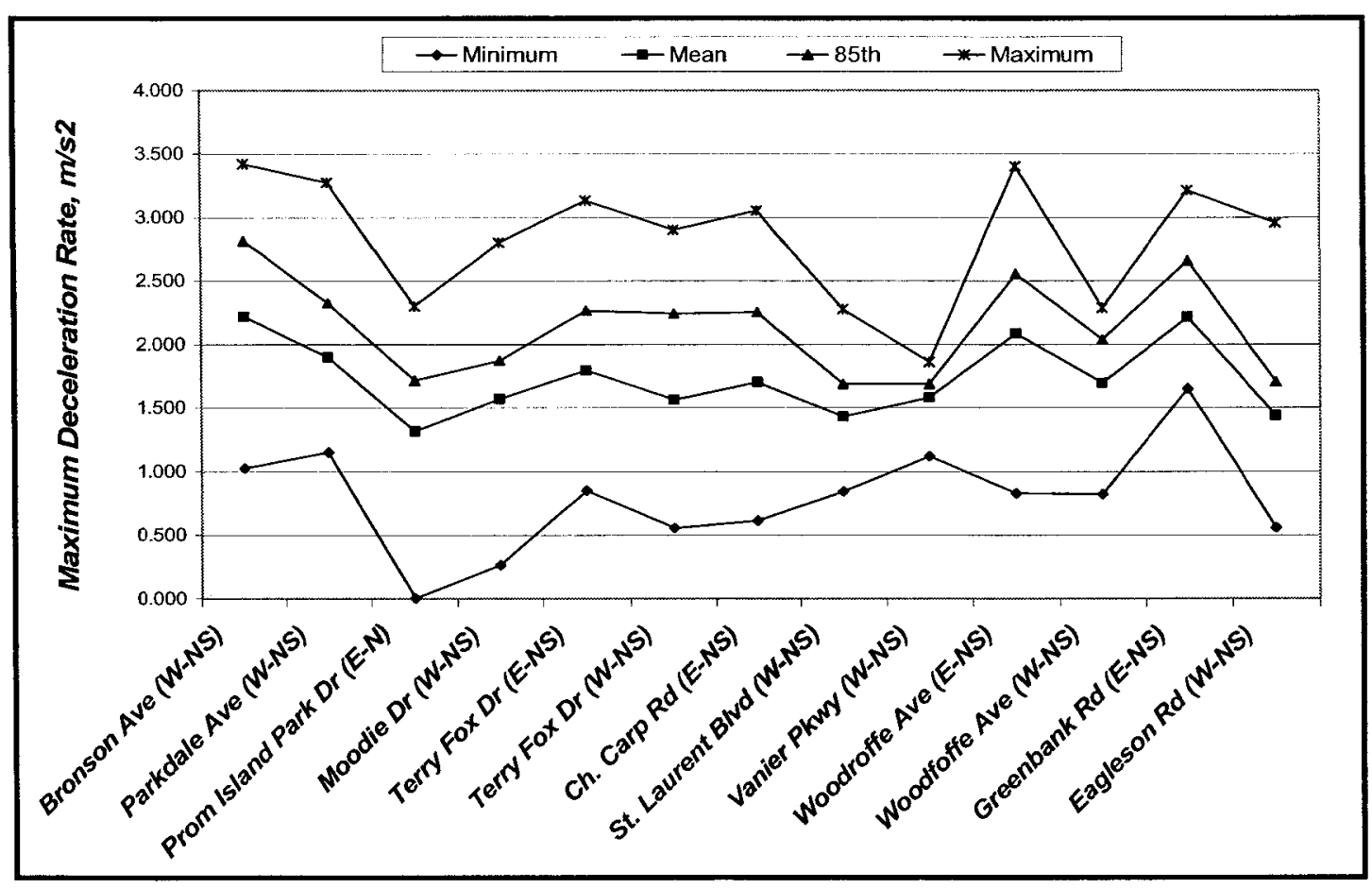

Figure 4.30: Distribution of Maximum Deceleration Rate on SCL

\subsubsection{Distribution of Effective Deceleration Distance}

The distribution of minimum, mean, $85^{\text {th }}$ percentile, and maximum effective deceleration distances used to decelerate on SCLs are shown in Figure 4.31. The effective deceleration distance among all sites in this study ranged from a shortest deceleration distance of $8.3 \mathrm{~m}$ at Island Park E-N to a longest deceleration distance of $576.8 \mathrm{~m}$ at Woodroffe E-NS. The $85^{\text {th }}$ and $95^{\text {th }}$ effective deceleration distances ranged from $39.1 \mathrm{~m}$ to $452.4 \mathrm{~m}$ with mean of $277.2 \mathrm{~m}$ and from $48.1 \mathrm{~m}$ to $514.3 \mathrm{~m}$ with a mean of $313.9 \mathrm{~m}$, respectively. Figure 4.32 shows a distribution comparison between $85^{\text {th }}$ percentile effective deceleration distance, $95^{\text {th }}$ percentile effective deceleration distance, mean effective deceleration distance, and length of limited type SCLs. 


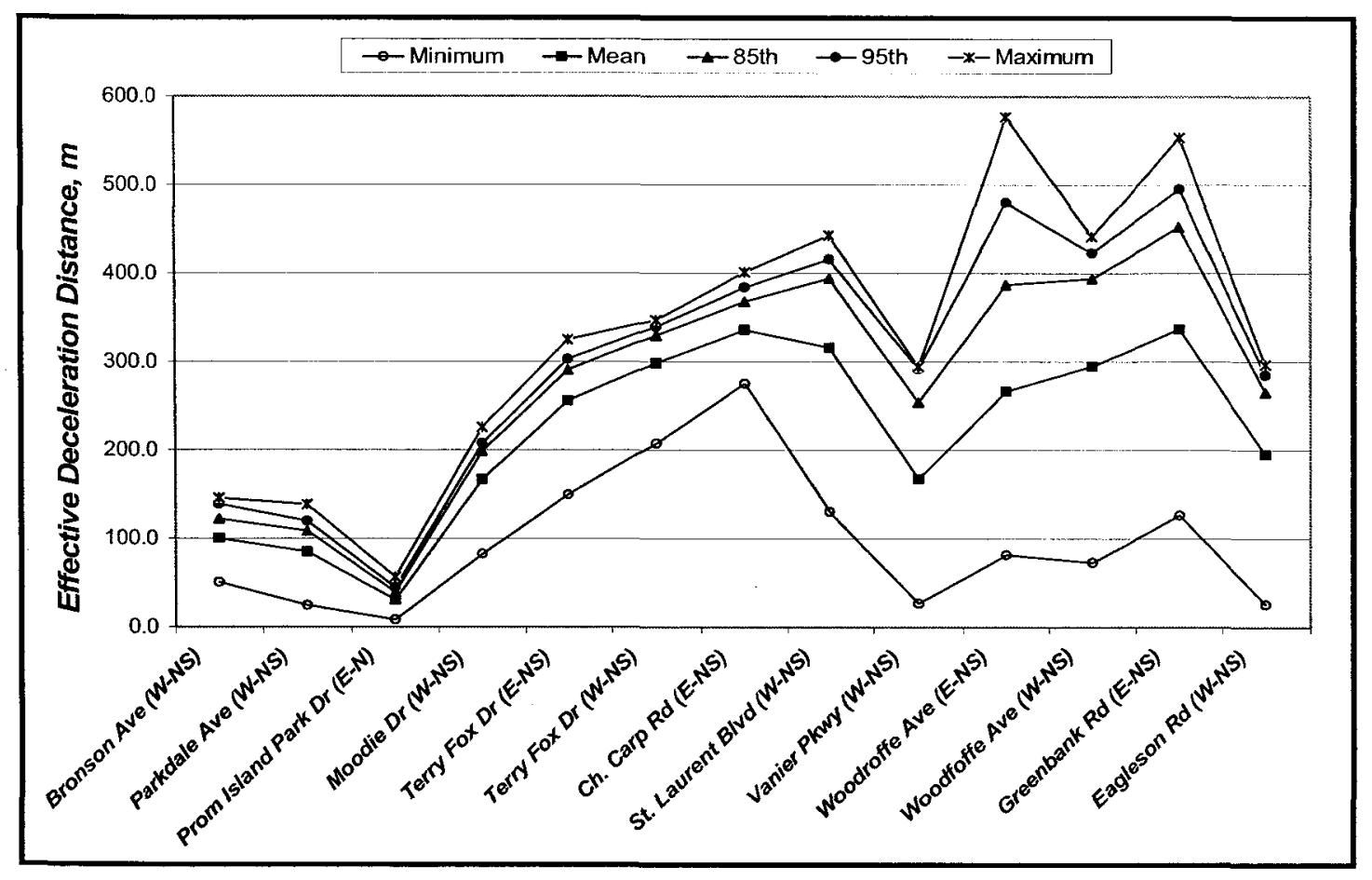

Figure 4.31: Distribution of Effective Deceleration Distance on SCL

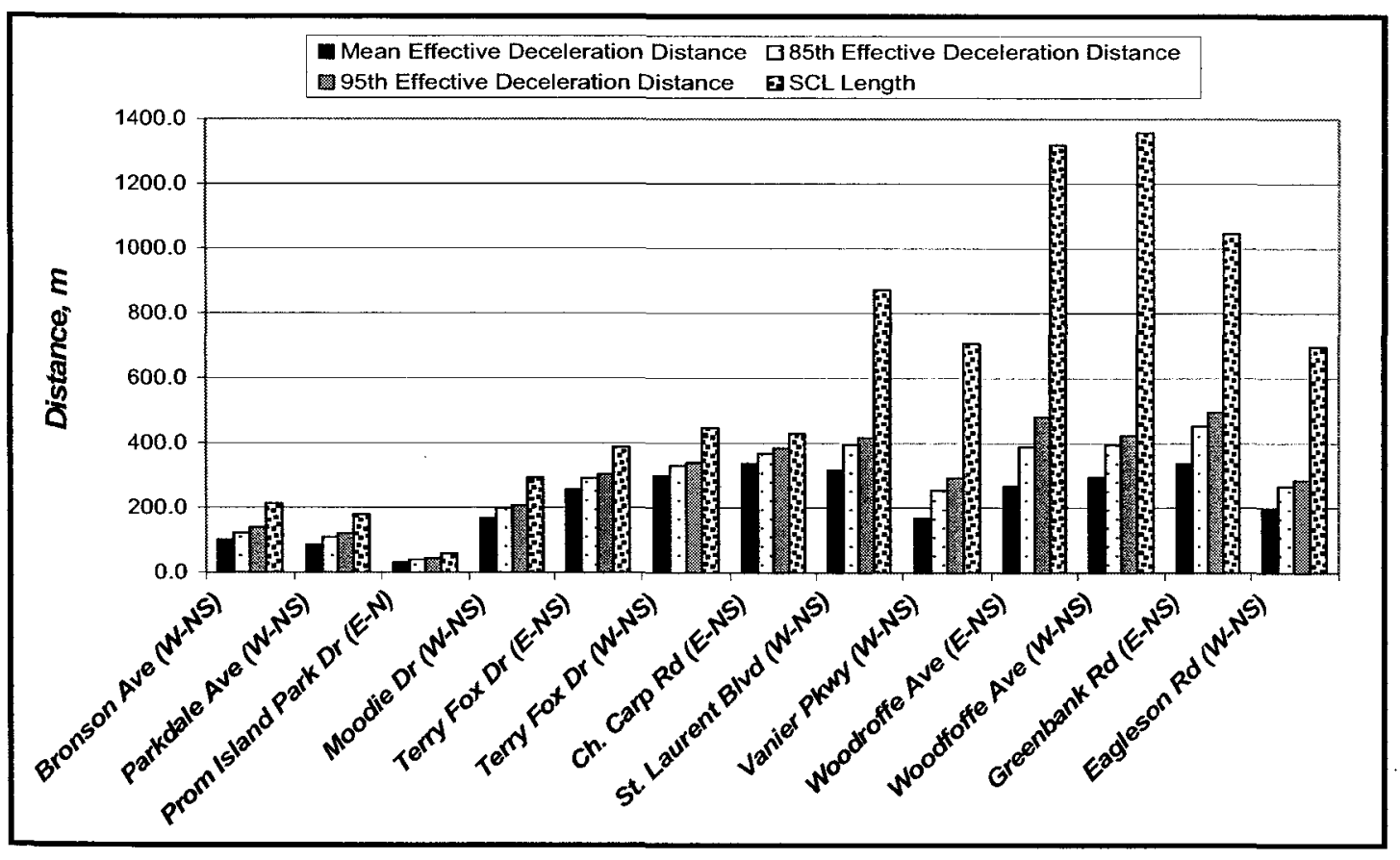

Figure 4.32: Frequency Distribution of $85^{\text {th }}, 95^{\text {th }}$, Mean Effective Deceleration Distance, and SCL Lengths 


\section{CHAPTER FIVE \\ 5.0 MODELLING AND DISCUSSION}

This chapter covers a description of the modelling attempts carried out in this study for developing models capable of predicting drivers' speeds, deceleration rates, and effective deceleration distances on freeway deceleration speed-change lanes (SCLs). In addition, the modelling attempts were performed to develop models capable of estimating the mean speed on the freeway adjacent right-most lanes (RLs). Several geometric and traffic parameters were modelled using multiple linear regression techniques to study their influence on seven driver behaviour measures (dependent variables) listed as follows:

- RL mean speed along the freeway diverge influence area, $V_{R L}$

- Diverge speed onto the deceleration SCL, $V_{\text {Diverge }}$

- Speed differential between RL mean speed and diverge speed, $\Delta V_{R L-D i v e r g e}$

- Gore speed at end of deceleration SCL $V_{\text {Gore }}$

- Overall deceleration rate on $\mathrm{SCL}, d_{\text {Overall }}$

- Maximum deceleration rate on SCL, $d_{M a x}$

- Effective deceleration distance used to decelerate from the diverge point to the end of the deceleration SCL, $D_{D e c}$

The driver behaviour parameters listed above were determined as the population's $85^{\text {th }}$ percentile value of targeted vehicles on selected study sites. In addition, the population's $95^{\text {th }}$ percentile effective deceleration distance was determined for modelling purposes. Modelling the $95^{\text {th }}$ percentile effective deceleration distance would help assess for the effective deceleration distance used by the larger portion of the population. Since 
length of the deceleration lane is the most important geometric element to evaluate driver behaviour on diverge areas, the $95^{\text {th }}$ percentile effective deceleration distance were modelled to help determine the adequate SCL length to be provided for deceleration behaviour as long as it is not constrained by right-of-way.

The modelling in this study was carried out using the SPSS 13.0 software. SPSS is a widely and commonly used statistics package for multiple linear regression analysis. The forward stepwise multiple regression modelling was the key method implemented for regression analysis in this study. The modelling procedure used were applied in a way that when the SPSS output shows more than one statistically significant predictor variable in modelling a dependant variable, the last predictor variable was removed to test for the relative significance of the other variables. This was repeated until the first predictor variable was left alone in the model. Afterwards, all the removed predictor variables, except for the first predictor, were re-entered to test for their significance with the same dependant variable. Repetitively, the whole procedure were completed to the point where no more predictor variables were left to be re-entered for modelling the same dependant variable (i.e. driver behaviour parameter).

The modelling phase included three different categories of length type of deceleration SCLs selected and used in this study. The first category considered only the limited length type SCLs and the second category considered the extended length type SCLs while the last category combined both limited and extended length type SCLs. The modelling attempts carried out to model for extended length type SCLs were not successful and no models were developed due to the limited number of data points used in the study for extended length type SCLs. On the other hand, the modelling attempts 
carried out for both the limited length type and combined SCLs were successful and resulted in 14 and 20 statistically significant predictive models, respectively.

In addition, the statistical package SAS 9.1 was used for the safety performance modelling attempts through the application of Negative Binomial (NB) regression analysis. Unfortunately, the modelling attempts performed to explicitly study the safety performance of freeway diverge areas using a five-year period (1998-2002) collision data were not successful and no models were developed. This was attributable to the limited number of data points (selected sites) used in this study. The dependent variables used in safety performance modelling were the two collision classes (safety parameters) prepared as: (1) the total number of collisions on freeway through segment in addition to the following deceleration SCL $\left(\mathrm{Col}_{T h D e c}\right)$, and (2) the number of collisions on freeway deceleration SCL only $\left(\mathrm{Col}_{D e c}\right)$. The safety modelling attempts considered the traffic data of the collision data period as well as the geometrics, speeds, and deceleration rates prepared for the selected sites in this study as the independent predictor variables.

Section 5.1 presents the modelling attempts for developing models capable of predicting freeway RL mean speed and Section 5.2 presents modelling attempts for diverge speed on freeway deceleration SCLs. Then, Section 5.3 presents the modelling attempts for speed differentials between the RL mean speed and diverge speed. Section 5.4 presents the modelling attempt for estimating the gore speed at the end of SCLs, whereas Section 5.5 covers the modelling attempt for predicting overall and maximum deceleration rates on freeway deceleration SCLs. Finally, Section 5.6 presents the modelling attempts for developing models capable of predicting the effective deceleration distance used on SCLs. 


\subsection{FREEWAY RIGHT-LANE MEAN SPEED MODELS}

The modelling attempts to develop significant models capable of predicting the freeway RL mean speed $\left(V_{R L}\right)$ along freeway deceleration SCL resulted in two significant models at $5 \%$ level of significance (based on the value of the F-statistic). These two models were associated with limited length type SCLs only. On the other hand, no models were developed for the combined (limited and extended length type) SCLs. The independent (explanatory) variables entered in the modelling were as follows:

- Length of deceleration SCL $\left(L_{D e c}\right.$ or $\left.L_{L i m}\right), \mathrm{m}$

- Transition length $\left(L_{t}\right), \mathrm{m}$

- SCL configuration code $\left(S C L_{C o n f}\right)$, taper type $=0$, parallel type $=1$

- $\quad$ SCL type code $\left(S C L_{\text {Type }}\right)$, limited type $=0$, extended type $=1$

- Ramp width $\left(\operatorname{Ramp}_{W}\right), \mathrm{m}$

- Ramp grade $\left(\operatorname{Ramp}_{G}\right)$, downgrade $=0$, upgrade $=1$

- Number of basic freeway lanes $\left(F_{W y_{B L}}\right)$

- Divergence angle $(\theta)$, degrees

- Ramp advisory speed $\left(A d v_{\text {Speed }}\right), \mathrm{km} / \mathrm{h}$

- $85^{\text {th }}$ percentile diverge speed $\left(V_{\text {Diverge }}\right), \mathrm{km} / \mathrm{h}$

- Ramp equivalent hourly traffic volume $\left(Q_{\text {Ramp }}\right), \mathrm{pc} / \mathrm{h} / \mathrm{ln}$

- RL-Upstream equivalent hourly traffic volume $\left(Q_{R L}\right), \mathrm{pc} / \mathrm{h} / \mathrm{ln}$

- RL-Gore equivalent hourly traffic volume $\left(Q_{R L-G o r e}\right), \mathrm{pc} / \mathrm{h} / \mathrm{ln}$

- $\quad \mathrm{RL}$-Average equivalent hourly traffic volume $\left(Q_{R L-A v e r a g e}\right), \mathrm{pc} / \mathrm{h} / \mathrm{ln}$

- Diverging equivalent hourly traffic volume $\left(Q_{\text {Diverging }}\right), \mathrm{pc} / \mathrm{h}$

- Ramp heavy vehicle percentage $\left(H V_{\text {Ramp }}\right), \%$ 
- $\quad$ RL-Upstream heavy vehicle percentage $\left(H V_{R L}\right), \%$

- $\quad$ RL-Gore heavy vehicle percentage $\left(H V_{R L-G o r e}\right), \%$

- RL-Average heavy vehicle percentage $\left(H V_{R L-A v e r a g e}\right), \%$

- Diverging heavy vehicle percentage $\left(H V_{\text {Diverging }}\right), \%$

In addition to those independent variables listed above, several transformations were considered in the modelling attempts. These transformations were performed on variables that did not satisfy the linearity test between the independent variable itself and the dependent variable. The transformation forms considered in the modelling attempts included inverse $(1 / X)$, base-10 logarithm $(\log X)$, square $\operatorname{root}(\sqrt{X})$, and power $\left(X^{2}\right)$ functions. Selection of the suitable transformation was decided when the transformed predictor variable showed better relationship with the dependant variable and has improved the predictive model in terms of statistical significance.

The SPSS was run after adding the diverge speed multiplied by the SCL configuration ( $\left.V_{\text {Diverge }} \times S C L_{C o n f}\right)$ variable to the predictor variables mentioned above to model for the RL mean Speed. Table 5.1 shows a summary for the two models developed for predicting the RL mean speed along limited length type SCLs with the $R^{2}$ values, $p$ values, t-statistics, and degrees of freedom $(d f)$. It should be noted that the data points for limited length type SCLs used in the modelling in this study were limited due to practical reasons as explained in Section 3.1.2. Therefore, the developed models for limited length type SCLs should be applied with caution. However, the limited type models that were developed in the study explained the drivers' diverging and deceleration behaviour limitations on freeway diverge areas with limited deceleration SCLs. 
Table 5.1: RL Mean Speed Predictive Models Summary for Limited SCLs

\begin{tabular}{|c|c|c|c|c|c|c|}
\hline Model & Independent Variables & Coefficients & $p$-value & $t$ & $d f$ & $R^{2}$ \\
\hline \multirow{2}{*}{1} & Constant & 97.762 & 0.000 & 68.175 & \multirow{2}{*}{5} & \multirow{2}{*}{0.953} \\
\cline { 2 - 6 } & $L_{\text {Lim }}$ & 0.046 & 0.000 & 10.115 & & \\
\hline \multirow{3}{*}{2} & Constant & 153.440 & 0.001 & 9.728 & \multirow{3}{*}{4} & \multirow{2}{*}{0.931} \\
\cline { 2 - 5 } & $V_{\text {Diverge }} S C L_{\text {Conf }}$ & 0.134 & 0.002 & 7.099 & & \\
\cline { 2 - 5 } & Log $Q_{R L \text {-Gore }}$ & -18.438 & 0.036 & -3.109 & & \\
\hline
\end{tabular}

The two models in Table 5.1 showed high coefficient of determination values $\left(R^{2}\right)$ of 0.953 and 0.931 , respectively. This means that the predictor variables explained most of the variability in the $85^{\text {th }}$ percentile RL mean speed. In the first model, the correlation showed that as the predictor variable (length of limited SCL including taper, $L_{\text {Lim }}$ ) increases, the RL mean speed increases. The length of the limited type SCL was the only predictor appeared in the model and which explained $95.3 \%$ of the variability in the dependant variable.

On the other hand, the second model revealed that the RL mean speed increases with increase in the diverge speed multiplied by the SCL configuration (V $V_{\text {Diverge }} \mathrm{x}$ $\left.S C L_{\text {Conf }}\right)$ and/or with decrease in the logarithm $(\log )$ function of RL traffic volume downstream the freeway diverge area $\left(Q_{R L-\text { Gore }}\right)$. The two predictor variables appeared in the model explained $93.1 \%$ of the variability in the freeway RL mean speed. The diverge speed multiplied by the SCL configuration variable has explained $76.3 \%$ of the variability in the dependant variable only by itself. Whereas, the $\log$ function of the RL traffic volume downstream the freeway diverge area explained $16.8 \%$ of the variability in the RL mean speed. 
The two predictive models developed for estimating the freeway RL mean speed along limited length type SCLs could be written as follows:

$$
\begin{aligned}
& V_{R L}=97.762+0.046 L_{\text {Lim }} \\
& V_{R L}=153.440+0.134 V_{\text {Diverge }} \times S C L_{\text {Conf }}-18.438 \log Q_{R L-\text { Gore }}
\end{aligned}
$$

In the first model (Equation 5.1), the increase in the available length of limited SCL would allow diverging vehicles to attain higher speeds on the freeway right lanes with high diverge speeds and hence, provide better operation on the freeway right lane. Short SCL lengths available for drivers to diverge from the freeway right lane onto the deceleration SCL perhaps would force diverging drivers to perform part of their deceleration behaviour in the freeway right lane. This would definitely influence the overall operational performance of the freeway and increases safety issues regarding the high speed disparities that might occur between drivers continuing through on the freeway right lane and those diverging off the freeway onto the deceleration SCL.

In Table 5.1, the second model (Equation 5.2) showed an important variable that captured the difference between taper and parallel SCLs with effect of diverge speed $\left(V_{\text {Diverge }} \mathbf{x} S C L_{\text {Conf }}\right)$. For instance, a good design would require zero difference between the freeway RL speed and the diverge speed. Therefore, concerning the second model (Equation 5.2), for a diverge speed equal to $100 \mathrm{~km} / \mathrm{h}$ (basic freeway speed limit) and a taper SCL, the estimated RL mean speed would be equal to $104.6 \mathrm{~km} / \mathrm{h}$. As the diverge speed decreases to be lower than the freeway RL speed for the same SCL configuration (taper), there will be a decrease in the estimated RL mean speed. Alternatively, with the same diverge speed $(100 \mathrm{~km} / \mathrm{h})$ but attained onto a parallel SCL, the estimated RL mean speed increase to be $114.3 \mathrm{~km} / \mathrm{h}$. This means that as the length of limited SCL increases 
the RL mean speed would also increase, as explained earlier regarding the first model in Table 5.1.

On the other hand, the RL traffic volume downstream the freeway diverge area variable, appeared in Equation 5.2, indicates the amount of traffic occupied the freeway right lane along the diverge area. As the $\mathrm{RL}$ traffic volume downstream the freeway diverge area increases, the high traffic on the freeway right lane will reduce the gaps between the travelling vehicles and hence, the freeway RL speed should decrease - as in rush hour traffic conditions. Finally, as shown and explained by the developed models, adequate length designs of limited SCLs with proper considerations of diverge speed and traffic volumes on the freeway right lane would enhance the operation at freeway diverge areas.

\subsection{DIVERGE SPEED MODELS}

Several significant models at $5 \%$ level of significance were developed to predict the diverge speed ( $V_{\text {Diverge }}$ ) for diverging vehicles onto freeway deceleration SCL. The independent variables entered in the modelling attempts were those listed in Section 5.1 combining to them the $85^{\text {th }}$ percentile RL mean speed and excluding traffic volumes downstream the freeway diverge area.

\subsubsection{Diverge Speed Models for Combined SCLs}

Five significant models were developed for predicting the diverge speed for combined (limited and extended length type) SCLs resulted. Table 5.2 shows a summary for two out of the five developed models with the $R^{2}$ values, $p$-values, t-statistics, and 
degrees of freedom $(d f)$. The other additional three models are available in Table D-1 in Appendix D.

Table 5.2: Diverge Speed Predictive Models Summary for Combined SCLs

\begin{tabular}{|c|c|c|c|c|c|c|}
\hline Model & Independent Variables & Coefficients & $p$-value & $t$ & $d f$ & $R^{2}$ \\
\hline \multirow{4}{*}{1} & Constant & -59.187 & 0.006 & -3.540 & & \\
\cline { 2 - 5 } & $V_{R L}$ & 1.486 & 0.000 & 7.292 & \multirow{3}{*}{9} & \multirow{2}{*}{0.953} \\
\cline { 2 - 5 } & $\log L_{\text {Dec }}$ & 10.151 & 0.015 & 3.016 & & \\
\cline { 2 - 5 } & $\log Q_{\text {Diverging }}$ & -10.029 & 0.048 & -2.293 & & \\
\hline \multirow{3}{*}{2} & Constant & 81.769 & 0.001 & 4.631 & \multirow{3}{*}{0.783} \\
\cline { 2 - 5 } & Log $L_{\text {Dec }}$ & 14.157 & 0.026 & 2.619 & \\
\cline { 2 - 5 } & $\theta$ & -1.347 & 0.028 & -2.565 & & \\
\hline
\end{tabular}

In Table 5.2, the two models are shown to be statistically significant at a 5\% level of significance with high $R^{2}$ value of 0.953 for the first model and a reasonable $R^{2}$ value of 0.783 for the second model. These two predictive models developed for estimating the diverge speed on combined SCLs could be written as follows:

$$
\begin{aligned}
& V_{\text {Diverge }}=-59.187+1.486 V_{R L}+10.151 \log L_{\text {Dec }}-10.029 \log Q_{\text {Diverging }} \\
& V_{\text {Diverge }}=81.769+14.157 \log L_{\text {Dec }}-1.347 \theta
\end{aligned}
$$

In Equation 5.3, three predictors; the freeway RL mean speed, log function of the deceleration SCL length (limited or extended), and log function of traffic volume diverging onto the deceleration SCL, explained $95.3 \%$ of the variability in the diverge speed. The first predictor has explained $67 \%$ of that variability only by itself and the second predictor explained $25.5 \%$ of the variability while the third and last predictor explained $2.7 \%$ of the variability in the diverge speed. The model showed that the diverge speed increases with the increase in both the freeway RL mean speed and log function of 
the deceleration SCL length, but increases with the decrease in the log function of the diverging traffic volumes from the freeway right lane onto the deceleration lane.

As the RL mean speed increases, the diverging vehicles are expected to follow the freeway flow speed and so their diverge speed should increase. However, the diverge speed is strongly influenced by the deceleration SCL length available for diverging vehicles. Hence, long deceleration SCL lengths should increase the diverge speed because of the enough available length for diverging drivers to use for deceleration. For instance, extended SCLs should not exhibit low diverge speeds, although sometimes, unfamiliar drivers misjudge the purpose of that lane as an extended deceleration lane and use it as the freeway right lane. This would indicate hazardous manoeuvres, as unfamiliar drivers, whom used the extended SCL as a right lane, would quickly merge left to their right lane as soon as they realize the SCL will terminate.

Nevertheless, the increase in the diverging traffic volumes would decrease the diverge speed due to the high capacity on the deceleration lane. During peak-hour periods, as the diverging traffic volume increases on the SCL the speed would decrease and hence, the traffic volume would control the diverging speed rather to be controlled by the freeway geometry. Adequate SCL lengths is therefore required to sustain a diverge speed close enough to the freeway RL mean speed at different traffic conditions for safe and efficient operation at the freeway diverge areas.

In Equation 5.4, the two predictors variables appeared to explain $78.3 \%$ of the variability in the diverge speed were the log function of the deceleration SCL length and the divergence angle at the physical nose of the exit ramp terminal. The length of the deceleration SCL (in the log transformation) explained $64 \%$ of that variability by itself, 
whereas the divergence angle explained $14.3 \%$ of the variability in the dependent variable $\left(V_{\text {Diverge }}\right)$. The second model shown in Table 5.2 revealed that good estimation of the diverge speed on deceleration SCLs (close as much as possible to the freeway flow speed) could be obtained by the coordination between the necessary length of deceleration SCL and the suitable divergence angle at the physical nose.

As explained earlier, the deceleration SCL length has strong influence on the diverge speed. On the other hand, the divergence angle is another key geometric element that has an influence on the diverge speed. For a certain SCL length, as the divergence angle increases the exiting vehicles would attain low speeds at the end of the deceleration SCL due to the large divergence at the gore area. Such low speeds at the end of the SCL would create diverging vehicles to queue on the deceleration SCL upstream the gore area and hence, influence the diverge speed of diverging vehicles. At the same time, the larger the divergence angle, the more the driver to expect tighter exit ramp geometry downstream the gore area.

\subsubsection{Diverge Speed Models for Limited SCLs}

Four significant models were developed for predicting the diverge speed for limited length type SCLs. Table 5.3 shows a summary of two out of the four developed models with the $R^{2}$ values, $p$-values, $\mathrm{t}$-statistics, and degrees of freedom $(d f)$. The additional two models are available in Table E-1 in Appendix E. 
Table 5.3: Diverge Speed Predictive Models Summary for Limited SCLs

\begin{tabular}{|c|c|c|c|c|c|c|}
\hline Model & Independent Variables & Coefficients & p-value & $t$ & $d f$ & $R^{2}$ \\
\hline \multirow{3}{*}{1} & Constant & 70.471 & 0.000 & 19.856 & \multirow{3}{*}{4} & \multirow{3}{*}{0.989} \\
\hline & $\sqrt{L_{\text {Lin }}}$ & 1.679 & 0.004 & 5.850 & & \\
\hline & $S C L_{\text {Conf }}$ & 11.050 & 0.013 & 4.230 & & \\
\hline \multirow{3}{*}{2} & Constant & 161.835 & 0.000 & 15.305 & \multirow{3}{*}{4} & \multirow{3}{*}{0.984} \\
\hline & $\theta$ & -2.860 & 0.000 & -15.500 & & \\
\hline & $\log Q_{\text {Ramp }}$ & -12.227 & 0.037 & -3.090 & & \\
\hline
\end{tabular}

In Table 5.2, the two models are shown to be statistically significant at a 5\% level of significance with high coefficient of determination values $\left(R^{2}\right)$. The predictor independent variables appeared in the two models explained more than $90 \%$ of the variability in the diverge speed on limited SCLs. These two predictive models could be written as follows:

$$
\begin{aligned}
& V_{\text {Diverge }}=70.471+1.679 \sqrt{L_{\text {Lim }}}+11.050 S C L_{\text {Conf }} \\
& V_{\text {Diverge }}=161.835-2.860 \theta-12.227 \log Q_{\text {Ramp }}
\end{aligned}
$$

In the first model (Equation 5.5), the square root function of limited SCL length was found to explain $94 \%$ of the variability in the diverge speed on limited deceleration SCLs. Although the normal form of the limited SCL length showed high correlation with the dependent variable explaining $93.8 \%$ of the variability, no mutual correlation was found to take place with another predictor variable. Alternatively, the SCL configuration appeared with the square root function of the limited SCL length to explain about $4.9 \%$ of the variability in the diverge speed.

The model in Equation 5.5 showed that the diverge speed increases with the increase in both the square root of limited SCL length and the SCL configuration. As explained earlier, the increase in the SCL length would provide more length for diverging 
vehicles to diverge off the freeway and this would allow them to attain higher diverge speed. Therefore, short SCL lengths would force diverging vehicles to perform part of the deceleration behaviour in the freeway right lane, indicating unsafe and inefficient operation at freeway diverge areas. A good design should adapt to the sense of the importance of adequate deceleration SCL lengths for increasing the safety of freeways.

Looking at the second model in Table 5.3 (Equation 5.6), two parameters; divergence angle and $\log$ traffic volume on the ramp (or the diverging traffic volume onto the limited SCL), explained $98.4 \%$ of variability in the diverge speed. The model showed that the diverge speed will increase with the decrease in both or any of the predictor variables. The divergence angles for taper length type SCLs selected in this study were higher than the angles associated with parallel length type SCLs. However, good design should provide flat divergence angles with taper length type SCLs, as recommended by the current design guides, to ease the diverging manoeuvre. High angles at the physical nose with short available deceleration length would definitely force drivers to deceleration on the freeway right lane, indicating hazardous locations. On the other hand, small diverge angles at the physical nose with adequate deceleration length would in return increase safety and operation at freeway diverge areas.

The second predictor variable in Equation 5.6 showed that as there is an increase in the ramp traffic volume (or the diverging traffic volume) of limited length type SCL, there would be a decrease in the diverge speed due to the high traffic diverging from the freeway right lane and occupying the limited length type deceleration lane. 


\subsection{SPEED DIFFERENTIAL MODELS}

Several significant models at $5 \%$ level of significance were developed to predict the speed differential ( $\Delta V_{R L-D i v e r g e}$ ) between the freeway $85^{\text {th }} \mathrm{RL}$ mean speed and the $85^{\text {th }}$ diverge speed. The independent variables used were those listed in Section 5.1 excluding the $85^{\text {th }}$ percentile diverge speed variable.

\subsubsection{Speed Differential Models for Combined SCLs}

Five significant models were developed to predict the speed differential for combined (limited and extended length type) SCLs. Table 5.4 shows a summary for one out of the five models with the $R^{2}$ value, $p$-value, $\mathrm{t}$-statistics, and degrees of freedom $(d f)$. The other four additional models are available in Table D-2 in Appendix D.

Table 5.4: Speed Differential Predictive Model Summary for Combined SCLs

\begin{tabular}{|c|c|c|c|c|c|c|}
\hline Model & Independent Variables & Coefficients & $p$-value & $t$ & $d f$ & $R^{2}$ \\
\hline \multirow{4}{*}{1} & Constant & 97.514 & 0.026 & 2.657 & & \\
\cline { 2 - 5 } & $L_{\text {Dec }}^{2}$ & $-7.1 \times 10^{-6}$ & 0.002 & -4.163 & \multirow{3}{*}{9} & \multirow{2}{*}{0.807} \\
\cline { 2 - 5 } & $L_{t}$ & -0.091 & 0.013 & -3.077 & & \\
\cline { 2 - 5 } & $\log Q_{R L}$ & -28.157 & 0.047 & -2.304 & & \\
\hline
\end{tabular}

In Table 5.4, the model has a good coefficient of determination value $\left(R^{2}\right)$ of 0.807. This predictive model developed for estimating the speed differential on combined SCLs may be written as follows:

$$
\Delta V_{R L-D i v e r g e}=97.514-7.1 \times 10^{-6} L_{D e c}^{2}-0.091 L_{t}-28.157 \log Q_{R L}
$$

From Equation 5.7, three predictor variables; power function of the length of the deceleration SCL, the length of transition (from end of SCL to controlling ramp curve), 
and $\log$ function of the freeway RL traffic volume upstream the diverge area, explained $80.7 \%$ of the variability in the speed differential for combined SCLs. The first predictor only explained $44.3 \%$ of that variability and the second predictor explained $25.1 \%$ of the variability while the third predictor explained $11.3 \%$ of the variability. The speed differential on deceleration SCL increases with the decrease in any of the there predictors appeared in the model.

As previously mentioned, the increase in the length of deceleration SCL attains high diverge speeds. High difference between freeway RL mean speed and the diverge speed is not preferable for good designs. Moreover, the length of the transition appeared in the model shows that the longer the available distance for the driver before approaching the controlling ramp curve, the higher the tendency of the driver to perform high diverge speeds close enough to the freeway flow speed. Similarly, as the traffic on the freeway RL upstream the diverge area increases, the traffic governs the driver behaviour and therefore, the flow speed of travelling vehicles on the freeway RL would decrease to reduce the chance of having speed differential. Hence, good design of deceleration SCLs could be applied using the model shown in Equation 5.7 as long as the speed differential is not greater then $\pm 10 \mathrm{~km} / \mathrm{h}$ - a consistency criterion that can be used based on Lamm et al. (1999). Additionally, speed differential greater than $\pm 10 \mathrm{~km} / \mathrm{h}$ and less than or equal $\pm 20 \mathrm{~km} / \mathrm{h}$ could be reflected as fair design, while speed differentials in excess of $\pm 20 \mathrm{~km} / \mathrm{h}$ indicate a poor design. 


\subsubsection{Speed Differential Models for Limited SCLs}

Two significant models were developed for estimating the speed differential for limited length type SCLs. Table 5.5 shows a summary of one of the two developed models with the $R^{2}$ value, $p$-value, t-statistics, and degrees of freedom $(d f)$. The other additional model is available in Table E-2 in Appendix E.

Table 5.5: Speed Differential Predictive Model Summary for Limited SCLs

\begin{tabular}{|c|c|c|c|c|c|c|}
\hline Model & Independent Variables & Coefficients & $p$-value & $t$ & $d f$ & $R^{2}$ \\
\hline \multirow{3}{*}{1} & Constant & 27.634 & 0.000 & 11.220 & \multirow{3}{*}{4} & \multirow{3}{*}{0.967} \\
\hline & $L_{\text {Lim }}$ & -0.041 & 0.001 & -8.914 & & \\
\hline & $Q_{R L \text {-Average }}$ & -0.011 & 0.017 & -3.929 & & \\
\hline
\end{tabular}

In Table 5.5, the model is shown to have a high coefficient of determination values $\left(R^{2}\right)$ of 0.967 . The predictive model developed for estimating the speed differential on limited SCLs could be written as follows:

$$
\Delta V_{R L-\text { Diverge }}=27.634-0.041 L_{\text {Lim }}-0.011 Q_{R L-\text { Average }}
$$

From Equation 5.8, the two variables; the length of the limited SCL and the freeway average $\mathrm{RL}$ traffic volume along the freeway diverge area, explained $96.7 \%$ of the variability in the speed differential on limited length type SCLs. The length of limited SCL explained $83.8 \%$ of that variability while the average RL traffic volume along the freeway diverge area explained the other $12.9 \%$ of the variability in the speed differential. The model showed similar relationship to the one obtained from Equation 5.7. The speed differential on limited length type SCLs increases with the decrease in both or any of the limited SCL length and the average RL traffic volume along the freeway diverge area. 
As previously mentioned, the increase in the length of the limited length type SCL would assist diverging drivers to attain high diverging speeds onto the deceleration SCLs. As the diverge speed increases, the speed differential between the freeway RL mean speed and that diverge speed will decrease, indicating good SCL length design. Concerning the second predictor in Equation 5.8, as the average RL traffic volume along the freeway diverge area increases the freeway flow speed will decrease to lessen the speed differential due to the high traffic on the roadway. The higher the traffic on the freeway right lane, the lesser the speed differential between the RL mean speed and the diverge speed - as the traffic would control the performance of travelling vehicles rather to be controlled by the freeway geometry.

\subsection{GORE SPEED MODELS}

Several significant models were developed for estimating the gore speed $\left(V_{\text {Gore }}\right)$ at the end of deceleration SCL. The explanatory variables entered in the modelling attempts for the gore speed were the variables listed in Section 5.1 excluding the freeway traffic volume and heavy vehicle percentages downstream the freeway diverge area.

\subsubsection{Gore Speed Models for Combined SCLs}

Two significant models were developed for estimating the gore speed at the end for deceleration SCL for combined (limited and extended length type) SCL. Table 5.6 shows the summary for one of the two developed models with the $R^{2}$ value, $p$-value, $\mathrm{t}$ statistics, and degrees of freedom $(d f)$. The other additional model is available in Table D-3 in Appendix D. 
Table 5.6: Gore Speed Predictive Model Summary for Combined SCLs

\begin{tabular}{|c|c|c|c|c|c|c|}
\hline Model & Independent Variables & Coefficients & $p$-value & $t$ & $d f$ & $R^{2}$ \\
\hline \multirow{3}{*}{1} & Constant & 120.298 & 0.000 & 26.484 & & \multirow{3}{*}{0.779} \\
\cline { 2 - 5 } & $\theta$ & -2.302 & 0.000 & -5.305 & \multirow{2}{*}{0.0 .35} \\
\cline { 2 - 5 } & $Q_{\text {Diverging }}^{2}$ & $-2.5 \times 10^{-5}$ & 0.043 & -2.351 & & \\
\hline
\end{tabular}

In Table 5.6, the model is shown to have a reasonable coefficient of determination value $\left(R^{2}\right)$ of 0.779 . This predictive model developed for estimating the gore speed at end of deceleration SCL could be written as follows:

$$
V_{\text {Gore }}=120.298-2.302 \theta-2.5 \times 10^{-5} Q_{\text {Diverging }}^{2}
$$

In the gore speed predictive model for combined SCLs (Equation 5.9), the divergence angle and the power function of diverging traffic volume from the freeway right lane onto the deceleration SCL together explained $77.9 \%$ of the variability in the dependant variable. The first predictor explained $64.3 \%$ of that variability by itself while the second predictor explained $13.6 \%$ of the variability. From Table 5.6, the gore speed at end of deceleration SCL increases with the decrease in both or any of the two predictors shown in the model. As the divergence angle at the end of the deceleration SCL at the physical nose increases, the gore speed of exiting vehicles decreases due to the high divergence in the geometry of the exit roadway. Moreover, as the diverging traffic volume increases onto the deceleration SCL, the speed on that relevant deceleration lane decreases and hence, influences the gore speed to decrease.

Good design of deceleration SCL should select a proper divergence angle at the physical nose in accordance to the geometry of the exit terminal downstream the gore area. In other words, the divergence angle at the physical nose of the exit ramp terminal should be selected to capture the geometrics of the transition and the ramp curve 
downstream the terminal nose to improve the traffic operations and safety at the freeway diverge areas. On the other hand, the increase in the diverging traffic volume would force exiting vehicles to slow their speed. Therefore, the gore speed at the end of the deceleration SCL would decreases due to the control of the traffic volume on the relevant SCL.

\subsubsection{Gore Speed Models for Limited SCLs}

Four significant models were developed to predict the gore speed $\left(V_{\text {Gore }}\right)$ at the end of deceleration SCL for limited length type. Table 5.7 shows a summary for two out of the four developed models with the $R^{2}$ values, $p$-values, t-statistics, and degrees of freedom $(d f)$. The other two additional models are available in Table E-3 in Appendix E.

Table 5.7: Gore Speed Predictive Models Summary for Limited SCLs

\begin{tabular}{|c|c|c|c|c|c|c|}
\hline Model & Independent Variables & Coefficients & p-value & $t$ & $d f$ & $R^{2}$ \\
\hline \multirow{3}{*}{1} & Constant & 67.023 & 0.000 & 17.093 & \multirow{3}{*}{4} & \multirow{3}{*}{0.945} \\
\hline & $L_{t}$ & 0.196 & 0.011 & 4.493 & & \\
\hline & $1 / \theta$ & 118.893 & 0.015 & 4.071 & & \\
\hline \multirow{3}{*}{2} & Constant & 80.398 & 0.000 & 15.190 & \multirow{3}{*}{4} & \multirow{3}{*}{0.933} \\
\hline & $\overline{L_{\text {Lim }}}$ & 0.091 & 0.002 & 7.102 & & \\
\hline & $Q_{\text {Ramp }}$ & -0.027 & 0.041 & -2.966 & & \\
\hline
\end{tabular}

The models listed above showed high $R^{2}$ with the predictor variables explaining more than $90 \%$ of the variability in $85^{\text {th }}$ percentile gore speed. In Table 5.7 , the first model showed that the gore speed at the end of the deceleration SCL increases with increase in the length of transition, and decreases with increase in the divergence angle. In the second model, it is evident that the gore speed is expected to increase with increase in the length of the limited length type SCL, and decrease with the increase in the traffic 
volume diverging, occupying, and exiting the freeway $\left(Q_{\text {Ramp }}\right)$. The two predictive models developed for estimating the SCL gore speed for limited length deceleration lanes could be written as follows:

$$
\begin{aligned}
& V_{\text {Gore }}=67.023+0.196 L_{t}+118.893 / \theta \\
& V_{\text {Gore }}=80.398+0.091 L_{\text {Lim }}-0.027 Q_{\text {Ramp }}
\end{aligned}
$$

In Equation 5.10, the length of the transition measured from the end of the deceleration SCL to the beginning of the controlling ramp curve explained $71.6 \%$ of the variability in the gore speed. In addition, the inverse function of the divergence angle correlated with the gore speed to explain $22.9 \%$ of the variability. From Equation 5.10 , the longer the transition length, the more distance is available for the driver to travel until reaching the ramp curve. Such long distance enables the driver to attain higher gore speeds at the end of deceleration SCL. For instance, fairly large radius of ramp curves would provide more distances for exiting vehicles to continue their exiting manoeuvre via exit ramp with an acceleration behaviour where applicable. On the other hand, the lager the divergence angle, the lower the gore speed. The divergence angle should indicate to exiting drivers the nature of the ramp geometry downstream the gore area of the exit terminal. Large divergence angle should indicate a sharp ramp curve is expected to be ahead with limited distances while flat divergence angle should reflect a fairly straight distances available after the end of the deceleration SCL.

In Equation 5.11, the length of limited SCL and ramp traffic volume explained $93.3 \%$ of the variability in the gore speed. The length of the deceleration SCL only explained $78.6 \%$ of that variability on its own, whereas the ramp traffic volume explained $14.7 \%$ of the variability in the dependent variable $\left(V_{\text {Gore }}\right)$. As explained earlier, the SCL 
length has a great effect on the diverge speed, which will in return affect the gore speed at the end of the SCL in response to the available distance for the driver to use for deceleration behaviour. Longer deceleration SCLs should increase the expected gore speed at the end of the SCL as there is enough distance for the driver to change his/her behaviour from deceleration to acceleration and vice visa while approaching the end of the SCL. This would allow the driver to perform most, if not all, of his/her deceleration behaviour on the available length of the SCL before its end and hence, the driver will attain higher gore speed. Again, as the traffic on the SCL increases, the flow speed will decrease and as a result, lower gore speeds are expected at the end of deceleration SCL due to the heavy traffic.

\subsection{DECELERATION RATE MODELS}

Several significant models were developed to estimate the overall deceleration rates ( $\left.d_{\text {Overall }}\right)$ on SCLs. On the other hand, one significant model was developed for predicting maximum deceleration rate $\left(d_{M a x}\right)$ for combined SCLs only. This indicates that the maximum deceleration rate on limited length type SCLs was more or less constant with an average maximum deceleration rate of $2.235 \mathrm{~m} / \mathrm{sec}^{2}$ for the limited SCLs selected in this study. This deceleration rate could be used as the maximum comfortable deceleration rate on limited length type SCLs. Nevertheless, the independent variables used were the same independent variables listed in Section 5.1 excluding the RL traffic volume and HV $\%$ downstream the diverge area as well as the $85^{\text {th }}$ percentile diverge speed. 


\subsubsection{Overall Deceleration Models for Combined SCLs}

Six significant models were developed to predict the overall deceleration rate $\left(d_{\text {Overall }}\right)$ on combined (limited and extended length type) SCLs. Table 5.8 summarises two out of the six developed models with the $R^{2}$ values, $p$-values, t-statistics, and degrees of freedom $(d f)$. The four additional developed models are available in Table D-4 in Appendix D.

Table 5.8: Overall Deceleration Predictive Models Summary for Combined SCLs

\begin{tabular}{|c|c|c|c|c|c|c|}
\hline Model & Independent Variables & Coefficients & $p$-value & $t$ & $d f$ & $R^{2}$ \\
\hline \multirow{3}{*}{1} & Constant & 6.050 & 0.003 & 4.120 & \multirow{3}{*}{9} & \multirow{3}{*}{0.730} \\
\hline & $\log Q_{R L \text {-Average }}$ & -1.691 & 0.008 & -3.361 & & \\
\hline & $1 / \theta$ & -3.145 & 0.016 & -2.945 & & \\
\hline \multirow{3}{*}{2} & Constant & 1.721 & 0.001 & 5.271 & \multirow{3}{*}{9} & \multirow{3}{*}{0.637} \\
\hline & $L_{t}$ & -0.005 & 0.015 & -3.004 & & \\
\hline & $Q_{R L}$ & -0.001 & 0.043 & -2.361 & & \\
\hline
\end{tabular}

The overall deceleration rate predictive models listed above showed reasonable $R^{2}$. In the first model, the correlation showed that the overall deceleration rate on SCLs increases with decrease in the RL average traffic volume along the freeway diverge area and/or with increase in the divergence angle at the physical nose. In the second model, the overall deceleration rate is expected to increase with decrease in the transition length and/or the RL traffic volume upstream the freeway diverge area. The two predictive models developed for estimating the overall deceleration rate on combined SCLs could be written as follows:

$$
\begin{aligned}
& d_{\text {Overall }}=6.05-1.691 \log Q_{R L-\text { Average }}-3.145 / \theta \\
& d_{\text {Overall }}=1.721-0.005 L_{t}-0.001 Q_{R L}
\end{aligned}
$$


In Equation 5.12, the log function of the RL average traffic volume and the inverse function of the divergence angle explained $73 \%$ of the variability in the overall deceleration rate. The first predictor explained $47 \%$ of that variability, while the second predictor explained the other $26 \%$ of the variability in the dependant variable. As explained earlier, the increase in the $\mathrm{RL}$ average traffic volume along the freeway diverge area will affect the freeway flow speed to decrease. Subsequently, the diverge speed would decrease and therefore, the overall deceleration rate will decrease on the SCL. Hence, during heavy traffic conditions, the freeway geometry is no longer controlling the driver behaviour rather than the traffic itself. On the other hand, as the divergence angle increases, the change in geometry at the exit gore area will force drivers to attain low gore speeds. This means higher overall deceleration rates. Hence, a good design of deceleration SCLs should consider the effect of both traffic and freeway geometry in order to improve the overall deceleration behaviour of drivers on SCLs for better operation at freeway diverge areas.

In Equation 5.13, the transition length and the traffic volume on freeway RL upstream the diverge area explained $63.7 \%$ of the variability in the overall deceleration rate, distributed as $41.2 \%$ and $22.5 \%$ for the two variables, respectively. The increase in the transition length would provide the drivers with more distance for the deceleration behaviour before travelling on the controlling ramp curve. As a result, the overall deceleration rate should decrease on the SCL. In other words, longer transition lengths would aid drivers to attain higher gore speeds and thus, the overall deceleration rate will decrease. Again, the increase in the traffic volume on the freeway RL upstream the diverge area would decrease the overall deceleration rate on SCLs. Therefore, the longer 
the distance available for the drivers and the higher the traffic on the freeway right lane, the lower the overall deceleration rate on the SCL.

From the two models shown in Table 5.8, a good design of deceleration SCLs should consider the effect of exit terminal geometry downstream the gore area in addition to the influence of traffic on overall deceleration behaviour of exiting vehicles to improve the operation and safety at freeway diverge areas.

\subsubsection{Overall Deceleration Models for Limited SCLs}

One significant model was developed for estimating the overall deceleration rate ( $\left.d_{\text {Overall }}\right)$ on limited length type SCLs. This model is shown in Table 5.9 with the $R^{2}$ value, $p$-value, t-statistics, and degrees of freedom $(d f)$.

Table 5.9: Overall Deceleration Predictive Model Summary for Limited SCLs

\begin{tabular}{|c|c|c|c|c|c|c|}
\hline Model & Independent Variables & Coefficients & $p$-value & $t$ & $d f$ & $R^{2}$ \\
\hline \multirow{3}{*}{1} & Constant & 7.220 & 0.009 & 4.761 & & \multirow{3}{*}{4} \\
\cline { 2 - 6 } & $L_{\text {Lim }}^{2}$ & $-3.3 \times 10^{-6}$ & 0.015 & -4.077 & \multirow{2}{*}{0.919} \\
\cline { 2 - 5 } & $\log Q_{R L \text {-Average }}$ & -2.113 & 0.016 & -3.998 & & \\
\hline
\end{tabular}

The model in Table 5.9 showed a high $R^{2}$ value with its predictor variables explaining more than $90 \%$ of the variability in overall deceleration rate for limited length type SCLs. The first predictor - power function of the length of limited SCL - explained only $59.6 \%$ of the variability, whereas the other predictor variable $-\log$ function of the average RL traffic volume along the freeway diverge area - explained $32.3 \%$ of the variability in the overall deceleration rate. The model showed that as the length of the limited SCL increases, the overall deceleration rate decreases. Similarly, as the traffic 
volume along the freeway diverge area increases, the overall deceleration rate on the limited length type SCL decreases. This predictive model developed for estimating the overall deceleration rate on limited SCLs could be written as follows:

$$
d_{\text {Overall }}=7.220-3.3 \times 10^{-6} L_{\text {Lim }}^{2}-2.113 \log Q_{R L-\text { Average }}
$$

In Equation 5.14, the increase in the length of the limited SCL would provide diverging drivers with more distance for deceleration behaviour from the point where they diverge onto the SCL to the end of the SCL. The longer the distance available for the drivers, the more time they have to decelerate comfortably and safely on the SCL. On the other hand, as the average RL traffic volume along the freeway diverge area increases, the freeway flow speed decreases and therefore, the diverge speed would decrease. Having an individual driver diverging with a low diverge speed (during heavy traffic conditions) onto the SCL, at that instant the distance available for the driver is more than enough to decelerate safely and comfortably on the SCL. Moreover, when the occupying traffic on the relevant SCL is lower than the freeway mainline, the driver will certainly perform acceleration behaviour until arriving to the end of the SCL.

\subsubsection{Maximum Deceleration Models for Combined SCLs}

One significant model was developed to predict the maximum deceleration rate $\left(d_{M a x}\right)$ on combined SCLs. Table 5.10 shows the developed model with the $R^{2}$ value, $p$ value, t-statistics, and degrees of freedom $(d f)$. 
Table 5.10: Maximum Deceleration Predictive Model Summary for Combined SCLs

\begin{tabular}{|c|c|c|c|c|c|c|}
\hline Model & Independent Variables & Coefficients & $p$-value & $t$ & $d f$ & $R^{2}$ \\
\hline \multirow{5}{*}{1} & Constant & 8.397 & 0.000 & 6.279 & \multirow{5}{*}{8} & \multirow{5}{*}{0.887} \\
\hline & $\log Q_{R L \text {-Average }}$ & -1.840 & 0.005 & -3.815 & & \\
\hline & $\Delta V_{R L-\text { Diverge }}$ & -0.044 & 0.002 & -4.597 & & \\
\hline & $H V_{R L \text {-Average }}$ & -0.045 & 0.005 & -3.838 & & \\
\hline & $S C L_{\text {Type }}$ & -0.318 & 0.049 & -2.325 & & \\
\hline
\end{tabular}

The maximum deceleration rate predictive model tabulated above showed a good coefficient of determination $\left(R^{2}\right)$ of 0.887 . In Table 5.10, the model showed that the maximum deceleration rate increases with the decrease in all or any of the four predictor variables appeared in the model; namely: (1) log function of the RL average traffic volume along the freeway diverge area, (2) speed differential, (3) average HV \% along the freeway diverge area, and (4) type of the deceleration SCL (limited or extended). The four predictor variables explained $88.7 \%$ of the variability in the maximum deceleration rate distributed as $48.7 \%, 19 \%, 13.4 \%$, and $7.6 \%$, respectively.

The model to estimate the maximum deceleration rate on combined SCLs could be written as follows:

$$
d_{\text {Max }}=8.4-1.84 \log Q_{R L-A v e r a g e}-0.04 \Delta V_{R L-D i v e r g e}-0.05 H V_{R L-A v e r a g e}-0.32 S C L_{\text {Type }}
$$

In Equation 5.15, the log function of the RL average traffic volume along the freeway diverge area, speed differential, average $\mathrm{HV} \%$ along the freeway diverge area, and the SCL type have the same effect on the maximum deceleration rate. The increase in the $\mathrm{RL}$ average traffic volume and/or the average $\mathrm{HV} \%$ along the freeway diverge area will decrease the freeway flow speed as well as the diverge speed and therefore, the maximum deceleration rate will be reduced. 
Similarly, as the speed differential increases with a positive sign, this means that the diverge speed is lower than the freeway RL mean speed and hence, the maximum deceleration rate will decrease on the SCL. On the other hand, when the diverge speed is higher than the freeway RL mean speed (negative speed differential), the maximum deceleration rate is expected to increase.

Finally, the longer the deceleration SCL, the more distance available for deceleration behaviour and so the maximum deceleration rate should decrease. This is illustrated through the SCL type variable with " 0 " code for limited length type SCL and "1" for extended length type SLC. Hence, this model should be in assistance to highway designers to estimate the maximum deceleration rate on SCLs. This model considers the effect of traffic (in terms of traffic volume and HV \%), speed, and freeway geometry to help in improving the deceleration behaviour of drivers on SCLs for better operation at freeway diverge areas.

\subsection{EFFECTIVE DECELERATION DISTANCE MODELS}

Two statistically significant models were developed for predicting the effective deceleration distances $\left(D_{D e c}\right)$ on SCLs. The independent variables listed in Section 5.1 excluding $85^{\text {th }}$ percentile diverge speed as well as the traffic volume and HV \% downstream the freeway diverge area were used for modelling attempts. As mentioned earlier, $85^{\text {th }}$ and $95^{\text {th }}$ percentile effective deceleration distances were determined and used in modelling attempts. In fact, $95^{\text {th }}$ percentile effective deceleration distances showed better correlation than the $85^{\text {th }}$ percentile effective deceleration distances in terms of the statistical significance ( $p$-value) and coefficient of determination $\left(R^{2}\right)$. 


\subsubsection{Effective Deceleration Distance Models for Combined SCLs}

One significant model was developed to predict the effective deceleration distance for combined SCLs. Table 5.11 summarises the developed model with the $R^{2}$ value, $p$ value, t-statistics, and degrees of freedom $(d f)$.

Table 5.11: Effective Dec. Distance Predictive Model Summary for Combined SCLs

\begin{tabular}{|c|c|c|c|c|c|c|}
\hline Model & Independent Variables & Coefficients & $p$-value & $t$ & $d f$ & $R^{2}$ \\
\hline \multirow{3}{*}{1} & Constant & 30.050 & 0.612 & 0.524 & & \multirow{3}{*}{0.814} \\
\cline { 2 - 6 } & $L_{\text {Dec }}$ & 0.306 & 0.000 & 6.393 & \multirow{2}{*}{0.00} & \\
\cline { 2 - 6 } & $H V_{R L \text {-Average }}$ & 8.558 & 0.050 & 2.232 & & \\
\hline
\end{tabular}

In Table 5.11, the predictive model for estimating the effective deceleration distance on limited and extended SCLs showed a good coefficient of determination $\left(R^{2}\right)$ of 0.814 , although the model's intercept was not statistically significant. The two predictor variables; length of the deceleration SCL and the RL average $\mathrm{HV} \%$ along the freeway diverge area, explained $81.4 \%$ of the variability in the dependant variable $\left(D_{D e c}\right)$. The length of the deceleration SCL variable explained $72.1 \%$ of the variability in, while RL average HV \% along the freeway diverge area explained the other $9.3 \%$ of the variability. The predictive model developed for estimating the effective deceleration distance on combined SCLs could be written as follows:

$$
D_{D e c}=30.05+0.306 L_{D e c}+8.558 H V_{R L-\text { Average }}
$$

The model in Equation 5.16 showed that the effective deceleration distance increases with the increase in both or any of the length of deceleration SCL and the RL average HV \% along the freeway diverge. The increase in the SCL length would provide more distance for diverging vehicles to use for deceleration operations. Such effective 
deceleration distance to be used by the diverging vehicles is required to be off the freeway mainstream. Hence, short SCLs would not offer adequate deceleration distances for safe and efficient operation at freeway diverge areas, allowing diverging vehicles to perform part of their deceleration behaviour in the freeway RL. On the other hand, as the percentage of HVs on right lane along the freeway diverge area increases, the freeway flow speed is expected to decrease and hence, diverging vehicles would favour an early diverge manoeuvre. Therefore, a good SCL length design that considers the effect of traffic composition is expected to improve the operation at freeway diverge areas.

\subsubsection{Effective Deceleration Distance Models for Limited SCLs}

One significant model was developed to estimate the effective deceleration distance on limited length type SCLs. The developed model is shown in Table 5.12 with the $R^{2}$ values, $p$-values, t-statistics, and degrees of freedom $(d f)$.

Table 5.12: Effective Dec. Distance Predictive Model Summary for Length SCLs

\begin{tabular}{|c|c|c|c|c|c|c|}
\hline Model & Independent Variables & Coefficients & $p$-value & $t$ & $d f$ & $R^{2}$ \\
\hline \multirow{2}{*}{1} & Constant & 51.793 & 0.004 & 5.045 & \multirow{2}{*}{5} & \multirow{2}{*}{0.989} \\
\cline { 2 - 5 } & $L_{\text {Lim }}^{2}$ & 0.002 & 0.000 & 20.889 & & \\
\hline
\end{tabular}

The effective deceleration distance predictive model shown in Table 5.12 had a high coefficient of determination $\left(R^{2}\right)$ of 0.989 . The effective deceleration distance on limited SCLs increases with increase in the power function of length of limited SCL, which explained all of the variability by itself. This predictive model could be written as follows:

$$
D_{D e c}=51.793+0.002 L_{\text {Lim }}^{2}
$$


In Equation 5.17, the power function of the limited length of SCL showed to have a strong effect on the effective deceleration distance expected to be utilized by diverging vehicles. As the length of limited length type SCL increases, the distance available for diverging vehicles to use for deceleration increases. The model in Equation 5.17 is applicable for limited length SCL (including taper) $\geq 60 \mathrm{~m}$ and $\leq 440 \mathrm{~m}-$ the limits of the limited length type SCLs collected in this study. It should be noted that, SCL lengths outer the range of 60 to $440 \mathrm{~m}$ would predict larger effective deceleration distances than the available length. However, AASHTO (2004) recommends a minimum deceleration length of $240 \mathrm{~m}$ for parallel length type SCLs as a desirable design that is expected to operate efficiently. The maximum $95^{\text {th }}$ percentile effective deceleration distance on limited SCLs was observed to be $386.6 \mathrm{~m}$ in this study. Adding a 90 meter taper area (as recommended by TAC 1999) to the maximum effective deceleration distance observed on limited sites would give SCL length of $476.6 \mathrm{~m}$. Accordingly, a $475 \mathrm{~m}$ limited SCL should be adequately enough for completing the deceleration behaviour in a comfortable and safe manner. 


\section{CHAPTER SIX}

\subsection{APPLICATION OF DEVELOPED MODELS}

This chapter presents application examples of the developed models. The developed models were useful tools to be used by highway designers and professionals in selecting proper geometry of deceleration SCLs and improve the diverging performance of freeways. However, it is worthy and necessary to mention that the developed models should be applied for values that lie within the limits of the geometrics and traffic volumes that were used to develop the models.

Six models were selected for application illustrations to predict the driver behaviour measures prepared in this study. These six models were for combined SCLs only. The rationale for not selecting any of the developed models for limited length type SCLs was because of the small values of the degrees of freedom associated with the models. The models selected for application illustrations had special characteristic that at least one of the predictor variables appeared in the model(s) is a design element that could be controlled by highway designers. The following sections present the application of each of the six selected models in the same order they are listed below.

- $V_{\text {Diverge }}=-59.187+1.486 V_{R L}+10.151 \log L_{\text {Dec }}-10.029 \log Q_{\text {Diverging }}$

- $\Delta V_{R L-\text { Diverge }}=97.514-7.1 \times 10^{-6} L_{D e c}^{2}-0.091 L_{t}-28.157 \log Q_{R L}$

- $V_{\text {Gore }}=120.298-2.302 \theta-2.5 \times 10^{-5} Q_{\text {Diverging }}^{2}$

- $d_{\text {Overall }}=6.05-1.691 \log Q_{R L-\text { Average }}-3.145 / \theta$

- $d_{\text {Max }}=8.4-1.84 \log Q_{R L-A v e r a g e}-0.04 \Delta V_{R L-D i v e r g e}-0.05 H V_{R L-A v e r a g e}-0.32 S C L_{\text {Type }}$

- $D_{\text {Dec }}=30.05+0.306 L_{D e c}+8.558 H V_{R L-A v e r a g e}$ 


\subsection{APPLICATION OF DIVERGE SPEED}

The application illustration of the selected model for estimating the diverge speed $\left(V_{\text {Diverge }}\right)$ on deceleration SCLs resulted in creation of several graphs as potential design aids for highway designers and professional. The model was developed to estimate the diverge speed or the deceleration SCL length $\left(L_{D e c}\right)$ to be provided while attaining the desired speed on the freeway right lane $\left(V_{R L}\right)$ and knowing the diverging traffic volume $\left(Q_{\text {Diverging }}\right)$. Figure 6.1 shows a series of graphs of the estimated diverge speed plotted against the length of the deceleration SCL for different diverging traffic volumes. Five graphs were created for different $\mathrm{RL}$ speeds on the freeway ranged from 80 to $120 \mathrm{~km} / \mathrm{h}$, and are presented next.

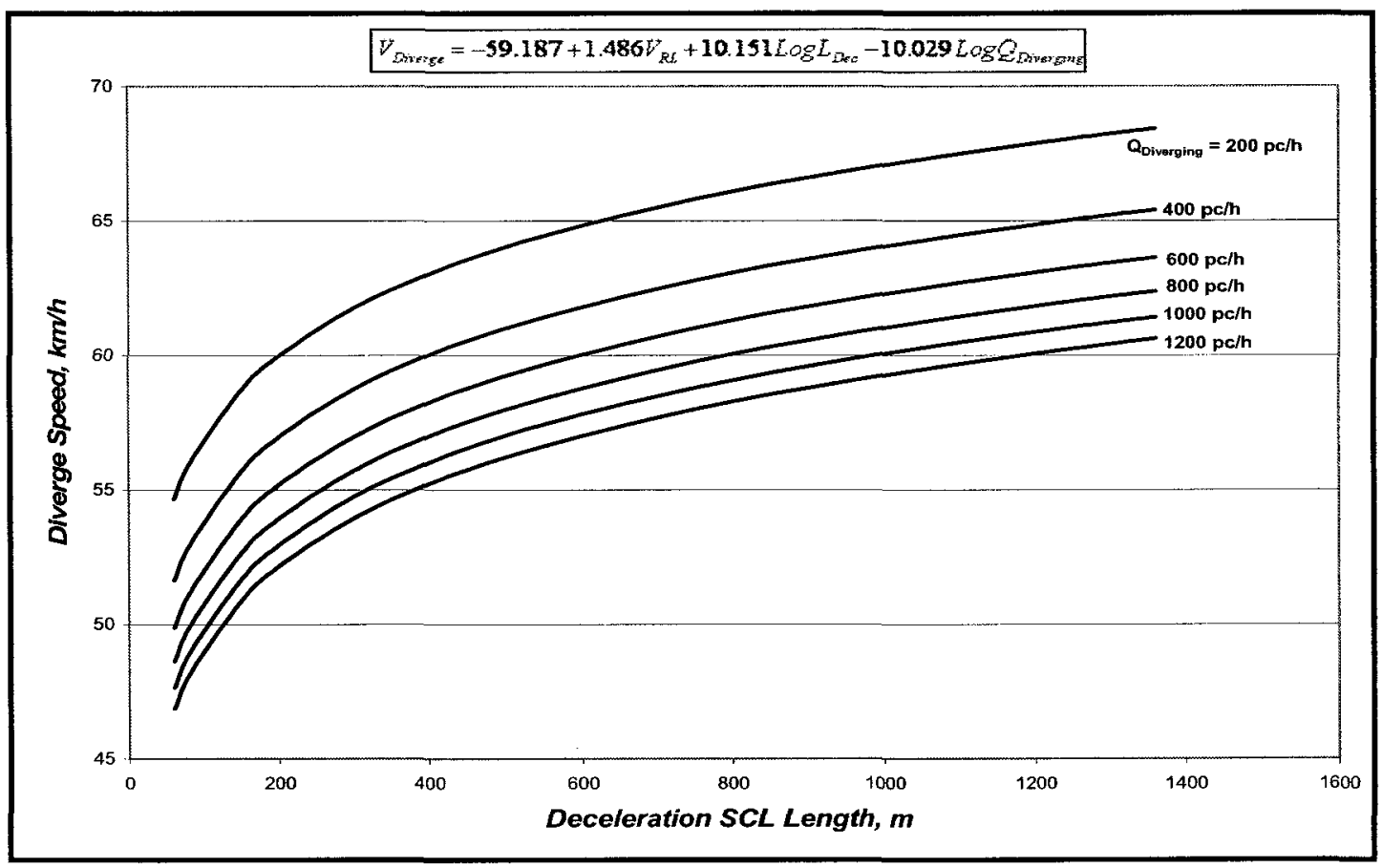

Figure 6.1.a: Application of Diverge Speed Based on Length of Deceleration SCL and Diverging Traffic Volume $\left(V_{R L}=80 \mathrm{~km} / \mathrm{h}\right)$ 


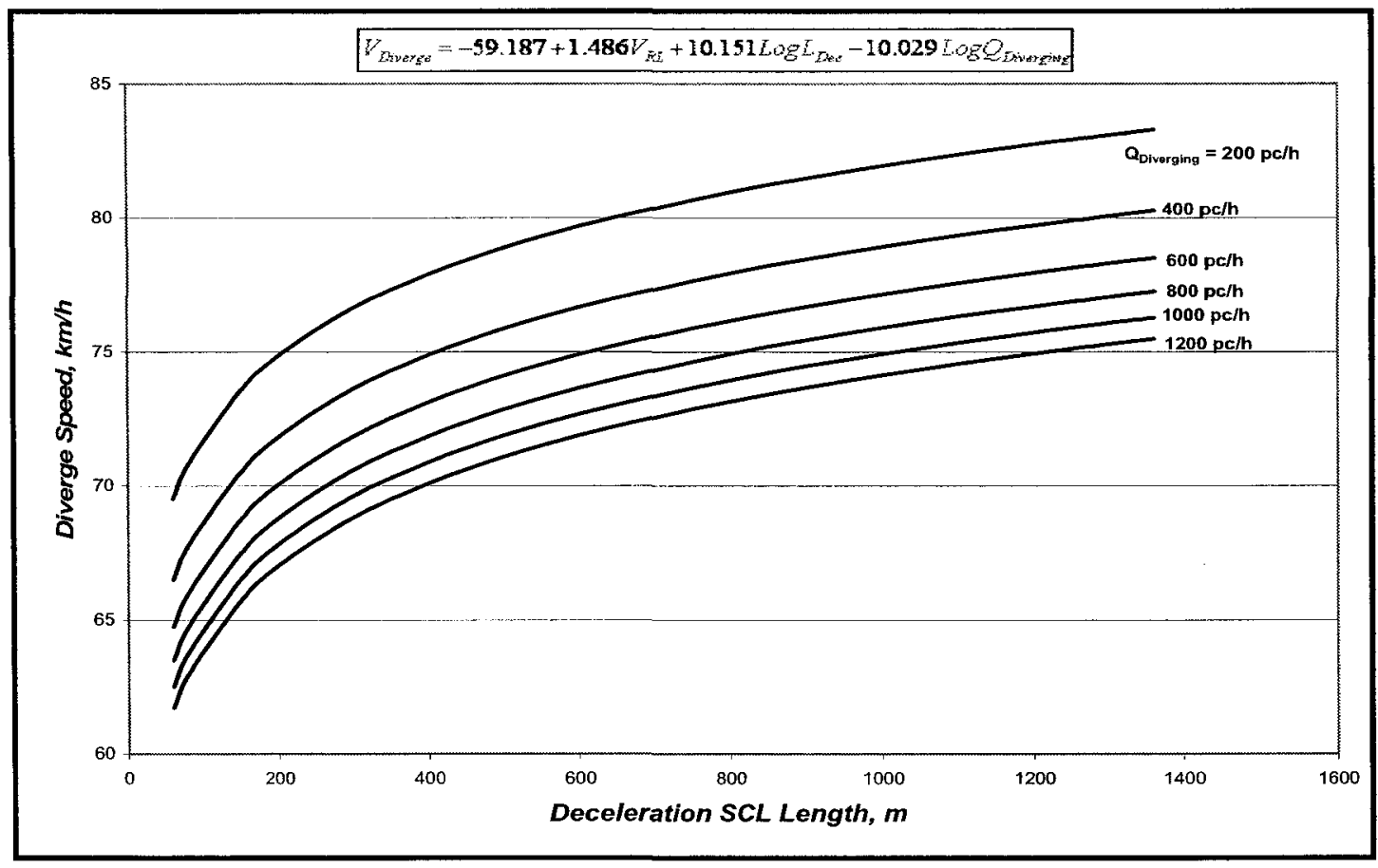

Figure 6.1.b: Application of Diverge Speed Based on Length of Deceleration SCL and Diverging Traffic Volume $\left(V_{R L}=90 \mathrm{~km} / \mathrm{h}\right)$

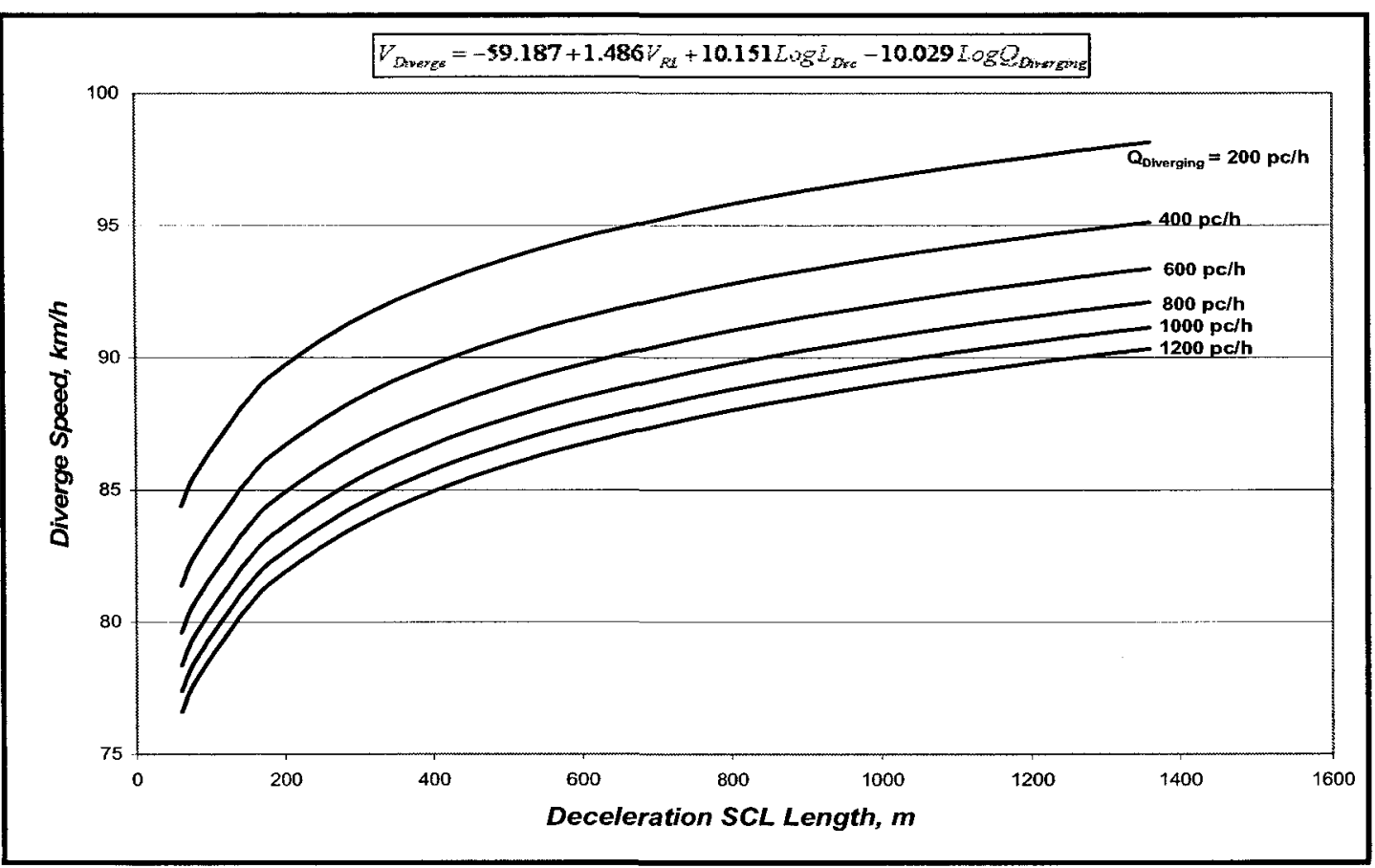

Figure 6.1.c: Application of Diverge Speed Based on Length of Deceleration SCL and Diverging Traffic Volume $\left(V_{R L}=100 \mathrm{~km} / \mathrm{h}\right)$ 


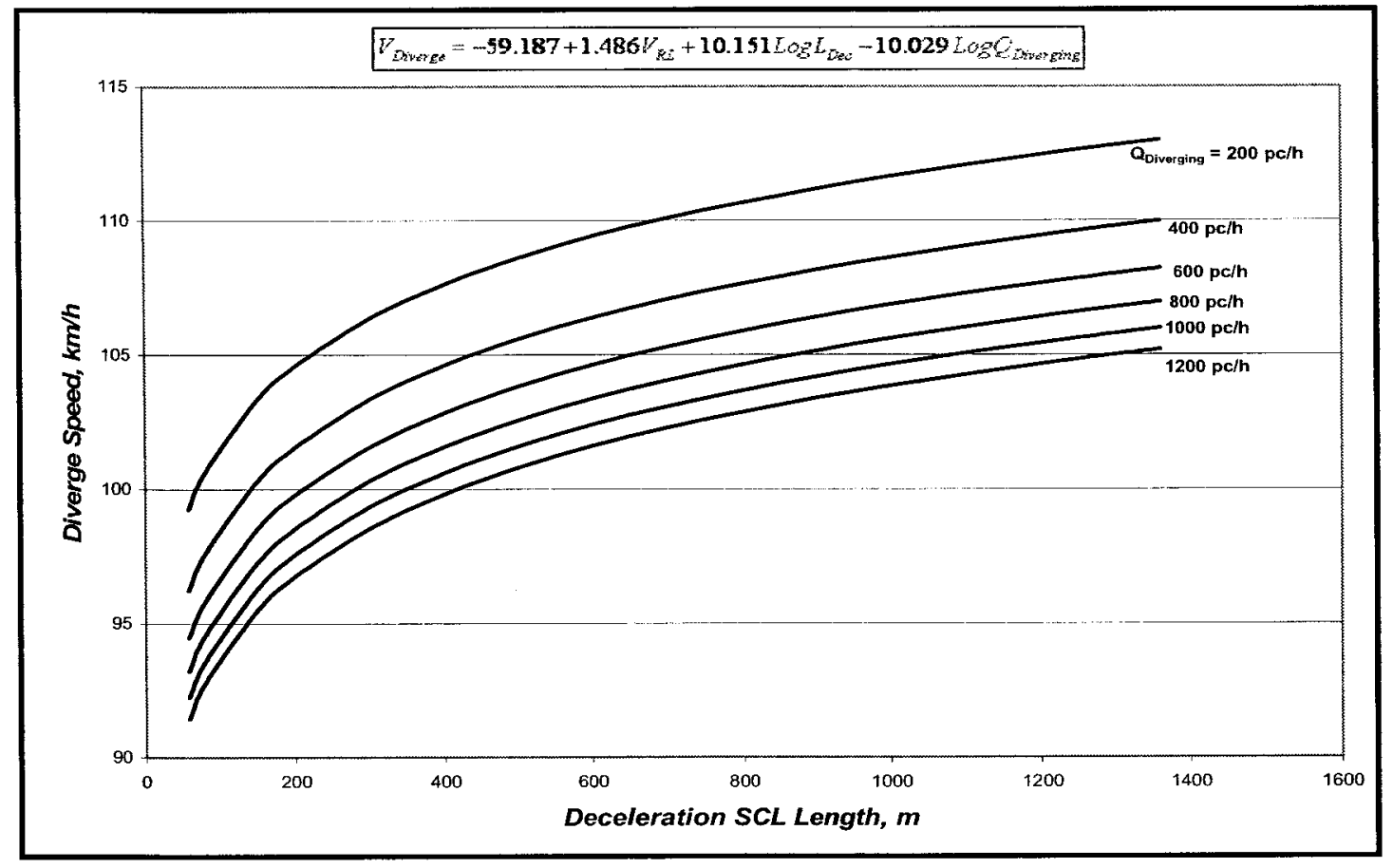

Figure 6.1.d: Application of Diverge Speed Based on Length of Deceleration SCL and Diverging Traffic Volume $\left(V_{R L}=110 \mathrm{~km} / \mathrm{h}\right)$

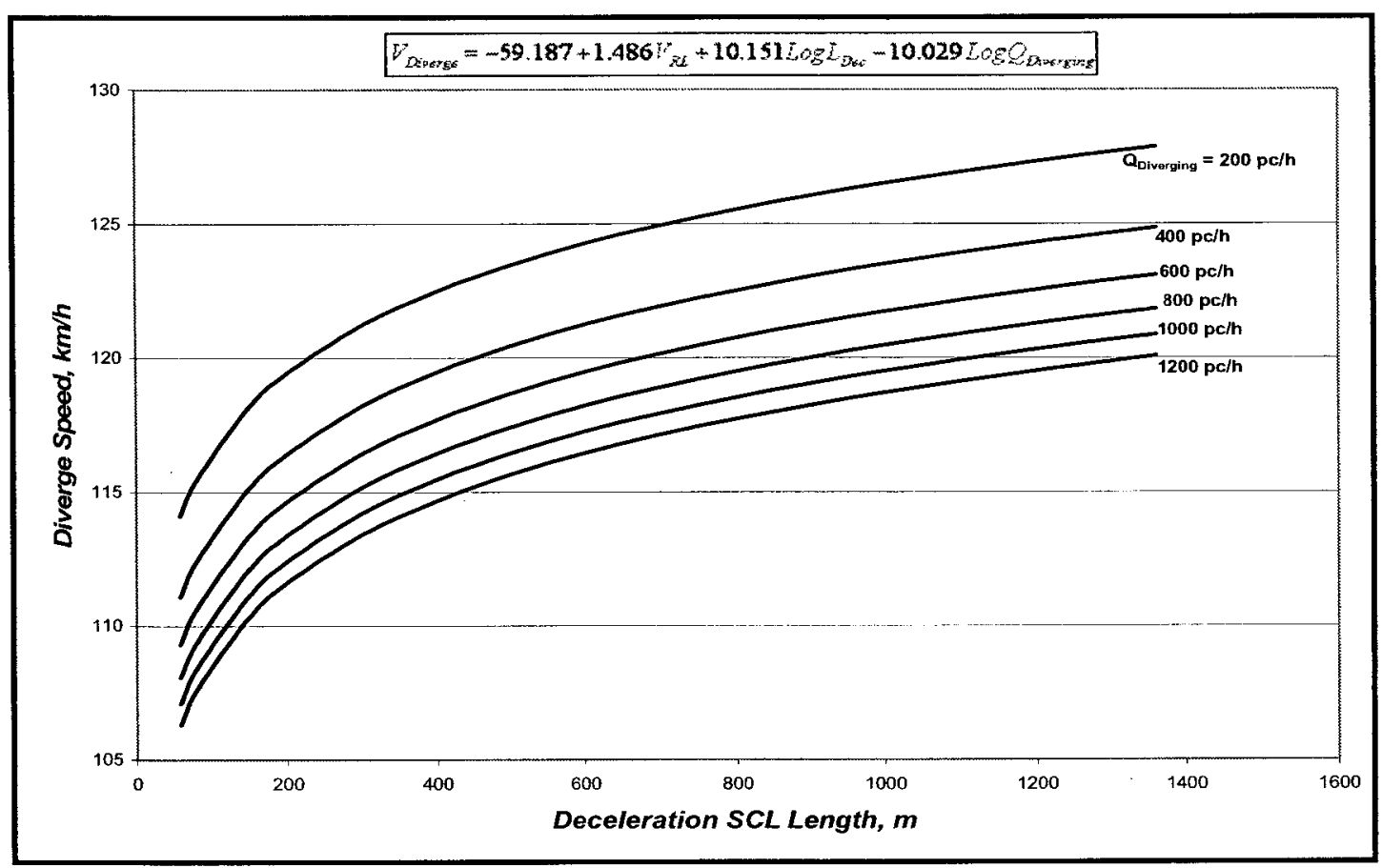

Figure 6.1.e: Application of Diverge Speed Based on Length of Deceleration SCL and Diverging Traffic Volume $\left(V_{R L}=120 \mathrm{~km} / \mathrm{h}\right)$ 
From the above graphs, knowing the freeway speed and the diverging traffic volume, the highway designer may estimate a desired diverge speed for a certain deceleration SCL length or estimate the necessary deceleration SCL length to attain a desired diverge speed. For instance, in Figure 6.1.c, while designing a deceleration SCL with a length of $475 \mathrm{~m}$, the expected diverge speed would be about $85.7 \mathrm{~km} / \mathrm{h}$ for a freeway RL mean speed of $100 \mathrm{~km} / \mathrm{h}$ and a diverging traffic volume of $1200 \mathrm{pc} / \mathrm{h}$. The expected diverge speed would decrease from 85.7 to about $82.7 \mathrm{~km} / \mathrm{h}$ while designing a shorter SCL of $240 \mathrm{~m}$ for the same RL speed of $100 \mathrm{~km} / \mathrm{h}$ and a diverging traffic volume of $1200 \mathrm{pc} / \mathrm{h}$. On the other hand, the increase in the freeway RL mean speed and/or decrease in the diverging traffic volume should increase the expected diverge speed for the same SCL length. Hence, the selection of a proper deceleration SCL length to attain a desired high diverge speed close enough to the RL mean speed for efficient traffic operations on freeway diverge areas could be executed through the application of the developed model (Figures 6.1.a through 6.1.e).

\subsection{APPLICATION OF SPEED DIFFERENTIAL}

The application illustration of the selected model for estimating the speed differential between freeway $\mathrm{RL}$ mean speed and the diverging speed ( $\Delta V_{R L \text {-Diverge }}$ ) on deceleration SCLs resulted in creation of several graphs. The created graphs are considered as potential design aids for highway designers and professional in predicting the speed differential. The developed model could be used to estimate the speed differential or the deceleration SCL length $\left(L_{D e c}\right)$ to be provided while knowing the transition length $\left(L_{t}\right)$ and the RL traffic volume upstream the freeway diverge area $\left(Q_{R L}\right)$. 
Figure 6.2 shows a series of graphs of the estimated speed differential plotted against the length of the deceleration SCL for different upstream RL traffic volumes. Four graphs were created for different transition lengths of $10,45,80$, and $115 \mathrm{~m}$.

Generally, although the SCL length provided is intended for deceleration behaviour, sometimes the diverging vehicles use the transition length in their deceleration performance. Such transition length was considered part of the deceleration SCL length in AASHTO (2004). However, the transition lengths in this study where not measured within the deceleration SCL lengths. Additionally, the increase in the transition length will provide more sight distance for the diverging vehicles from the point they diverge to the beginning of the controlling ramp curve at the physical nose of the exit terminal.

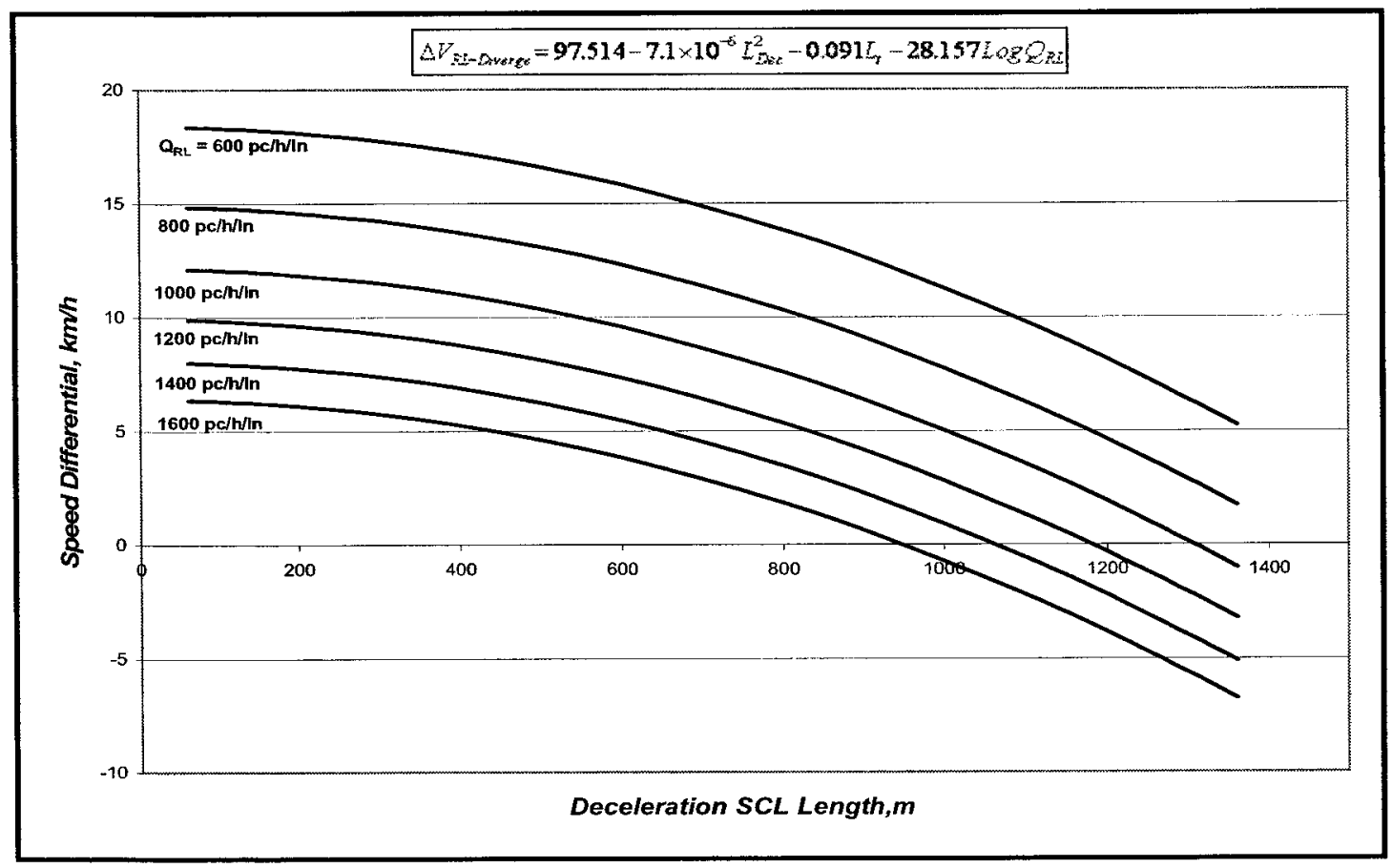

Figure 6.2.a: Application of Speed Differential Based on Length of Deceleration SCL and RL Average Traffic Volume $\left(L_{t}=10 \mathrm{~m}\right)$ 


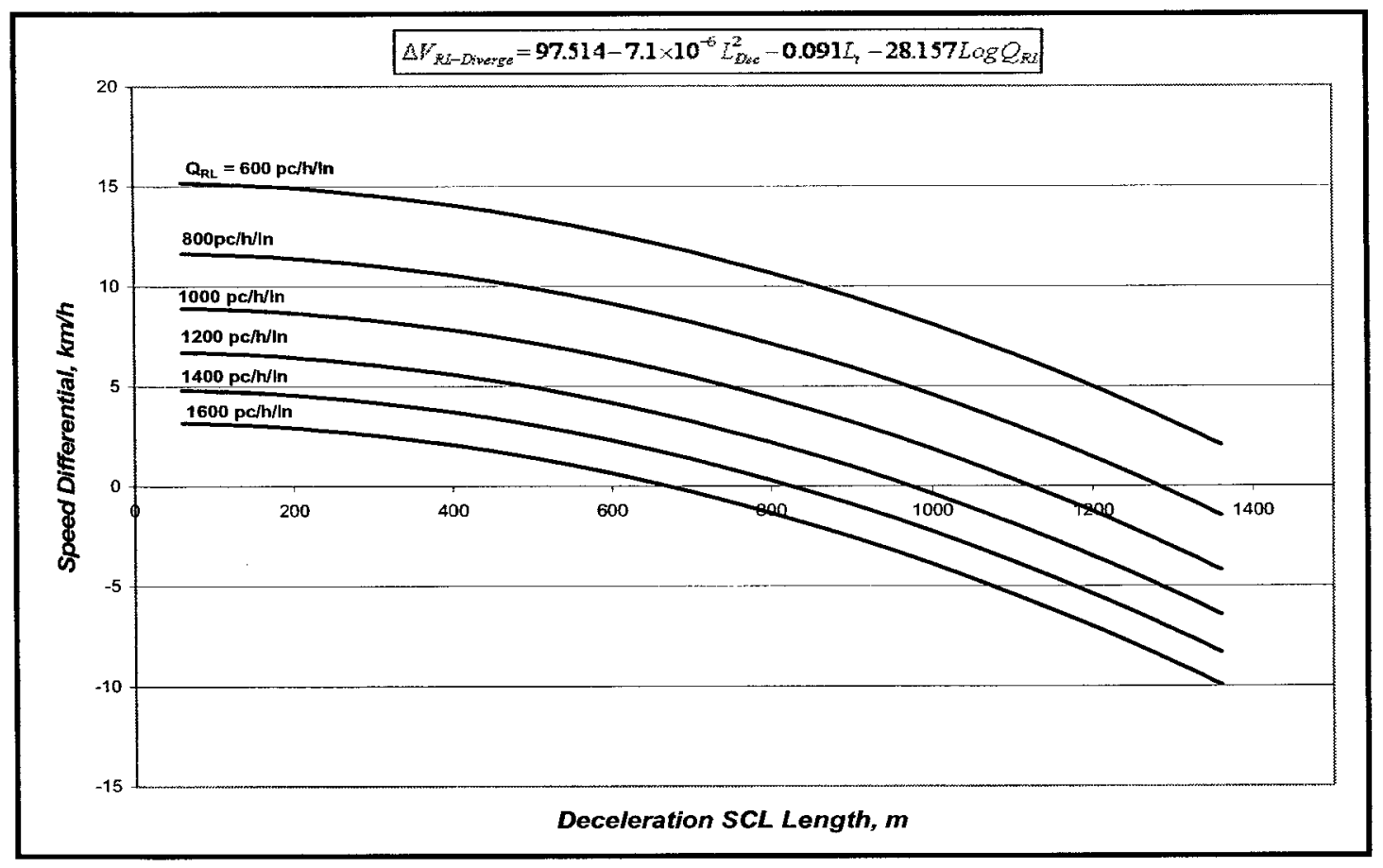

Figure 6.2.b: Application of Speed Differential Based on Length of Deceleration SCL and RL Average Traffic Volume $\left(L_{t}=45 \mathrm{~m}\right)$

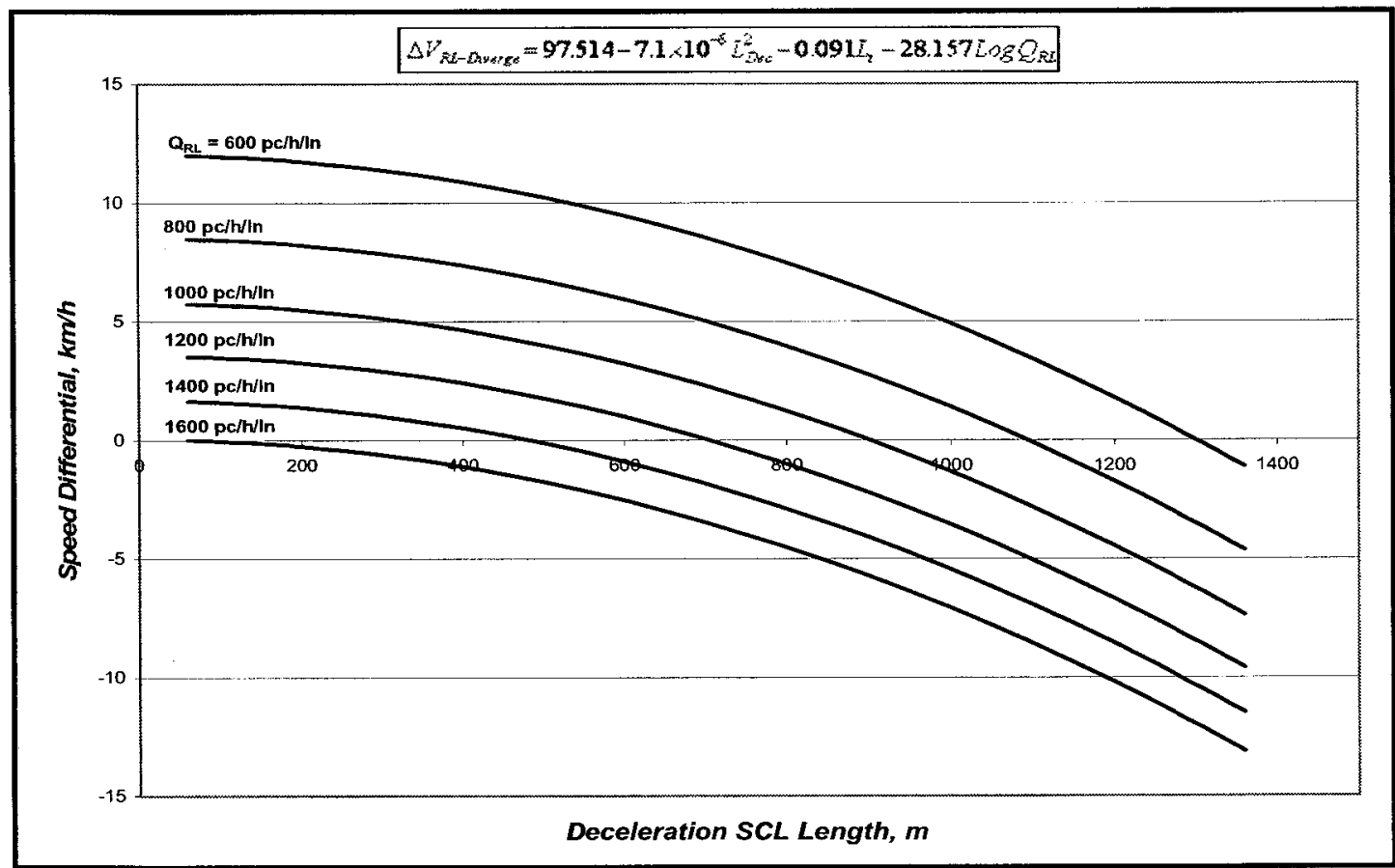

Figure 6.2.c: Application of Speed Differential Based on Length of Deceleration SCL and RL Average Traffic Volume $\left(L_{t}=80 \mathrm{~m}\right)$ 


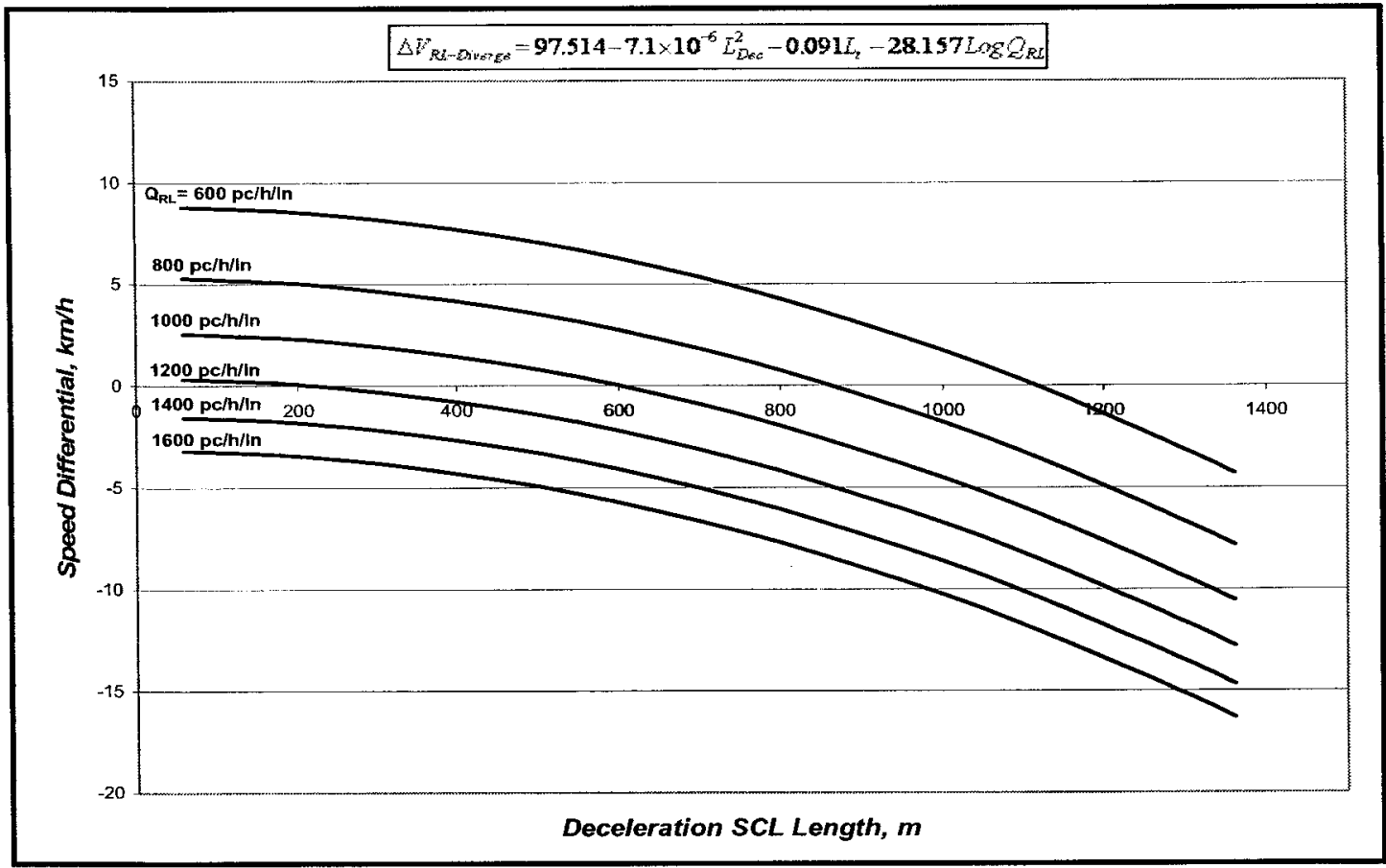

Figure 6.2.d: Application of Speed Differential Based on Length of Deceleration SCL and RL Average Traffic Volume $\left(L_{t}=115 \mathrm{~m}\right)$

In Figures 6.2.a through 6.2.d, the highway designer could predict the speed differential between the freeway RL mean speed and the diverge speed while knowing the RL traffic volume upstream the freeway diverge area, deceleration SCL length, and the transition length. Designing deceleration SCLs that would produce high speed differentials between the freeway RL mean speed and the diverge speed should be neglected. Such poor design reflects hazardous location due to the speed disparities between diverging and freeway through vehicles.

Knowing the transition length to be provided and the RL traffic volume upstream the freeway diverge area, the highway designer could predict the speed differential for different deceleration SCL lengths. Alternatively, the designer could still use this model to estimate the necessary deceleration SCL length to produce a desired speed differential. 
For example, in Figure 6.2.b, the expected speed differential for a transition length of 45 $\mathrm{m}$ and a RL traffic volume upstream the diverge area of $1400 \mathrm{pc} / \mathrm{h} / \mathrm{ln}$ would be $3.2 \mathrm{~km} / \mathrm{h}$ while designing a deceleration limited SCL of $475 \mathrm{~m}$ in length. Whereas, this speed differential would decrease to be $-5.4 \mathrm{~km} / \mathrm{h}$ when designing an extended deceleration SCL of $1200 \mathrm{~m}$ in length for the same transition length of $45 \mathrm{~m}$ and same upstream RL traffic volume of $1400 \mathrm{pc} / \mathrm{h} / \mathrm{ln}$. On the other hand, the speed differential would increase with decrease in the transition length and/or the RL traffic volume upstream the freeway diverge area. To provide longer transition length on the compensation of short deceleration SCLs might not be in good assistance to diverging vehicles to decelerate on the relatively short deceleration SCL provided. Rather, they might perform part of the deceleration behaviour on the freeway right lane that would lower the operation efficiency and safety on freeway diverge areas. Hence, the designer should harmonize between the length to be provided for both the deceleration SCL and transition to improve the traffic operation and safety on freeway diverge area.

\subsection{APPLICATION OF GORE SPEED}

The application illustration of the selected model for estimating the gore speed $\left(V_{G o r e}\right)$ at the end of deceleration SCLs related the divergence angle $(\theta)$ at the physical nose and the diverging traffic volumes $\left(Q_{\text {Diverging }}\right)$ as shown in Figure 6.3. The model was plotted to be used as a tool by highway designers and professional for estimating the gore speed while knowing the divergence angle and the diverging traffic volume on the deceleration SCLs. 


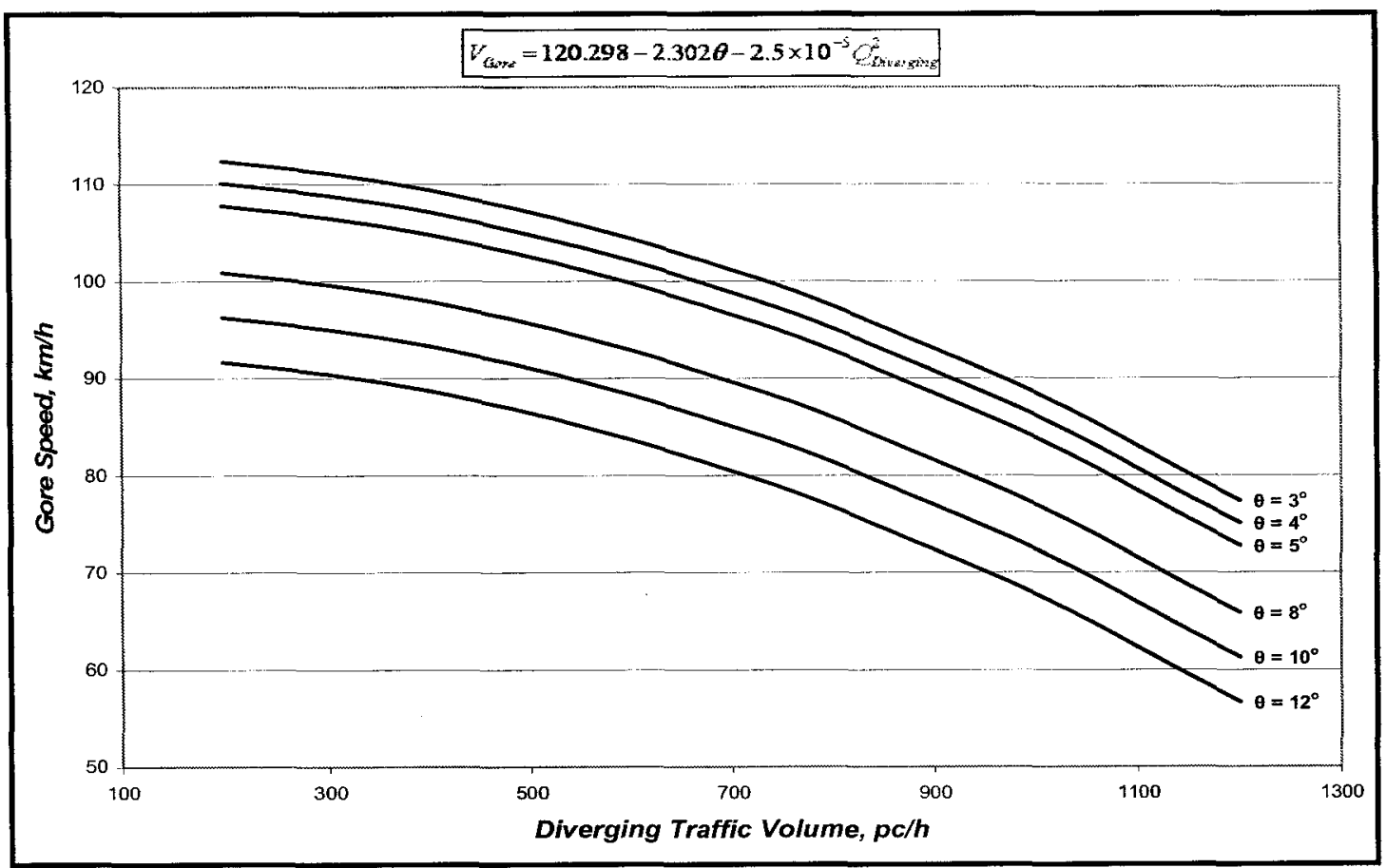

Figure 6.3: Application of Gore Speed Based on Diverging Traffic Volume and Divergence Angle

The gore speed at the end of the deceleration SCL is highly dependant on the diverge speed. As shown in Section 5.7.1, there is a relationship between the diverge speed and the deceleration SCL length. However, the SCL length showed impractical correlation with the gore speed during the modelling attempts. Nevertheless, in Figure 6.3 and for a particular deceleration SCL length, the expected gore speed at the end of the SCL would be $88.4 \mathrm{~km} / \mathrm{h}$ for a diverging traffic volume of $1000 \mathrm{pc} / \mathrm{h}$ and designing a divergence angle of $3^{\circ}$ at the physical nose of the exit terminal. The gore speed is expected to decrease from 88.4 to $72.3 \mathrm{~km} / \mathrm{h}$ when the divergence angle increased to $10^{\circ}$ but for the same diverging traffic volume of $1000 \mathrm{pc} / \mathrm{h}$. The divergence angle should not be enlarged when providing long and flat ramp curves following the deceleration SCL as this would reduce the gore speed at the end of SCL, which is not necessary in such 
case(s). On the other hand, large divergence angle would be preferred while providing short and flat or sharp ramp curves. However, this would necessitate the design of adequate deceleration SCL length to allow for efficient traffic operations and proper deceleration behaviour before approaching the ramp curve. Therefore, consistency between exit ramp different geometrics (SCL length, divergence angle, and ramp curve) that allow the diverging vehicles to attain suitable gore speed would improve traffic operations on freeway diverge areas.

Additionally, the model illustrated in Figure 6.3 showed the effect of traffic volume on gore speed at end of SCL. As the diverging traffic volume increases, the gore speed is expected to increase. For instance, the expected gore speed would be $75.1 \mathrm{~km} / \mathrm{h}$ for designing a deceleration SCL with a divergence angle of $4^{\circ}$ with $1200 \mathrm{pc} / \mathrm{h}$ diverging traffic volume onto the relevant SCL. For the same divergence angle, the estimated gore speed at the end of the SCL will increase to be about $110.1 \mathrm{~km} / \mathrm{h}$ when the diverging traffic decline to $200 \mathrm{pc} / \mathrm{h}$.

\subsection{APPLICATION OF OVERALL DECELERATION RATE}

The application illustration of the selected model for estimating the overall deceleration rate $\left(d_{\text {Overall }}\right)$ on deceleration SCLs related the divergence angle $(\theta)$ at the physical nose and the RL average traffic volumes $\left(Q_{R L \text {-Average }}\right)$ as shown in Figure 6.4. The model was plotted as a potential design aid for estimating the overall deceleration rate while knowing the divergence angle and the $\mathrm{RL}$ average traffic volume along the freeway diverge area. 


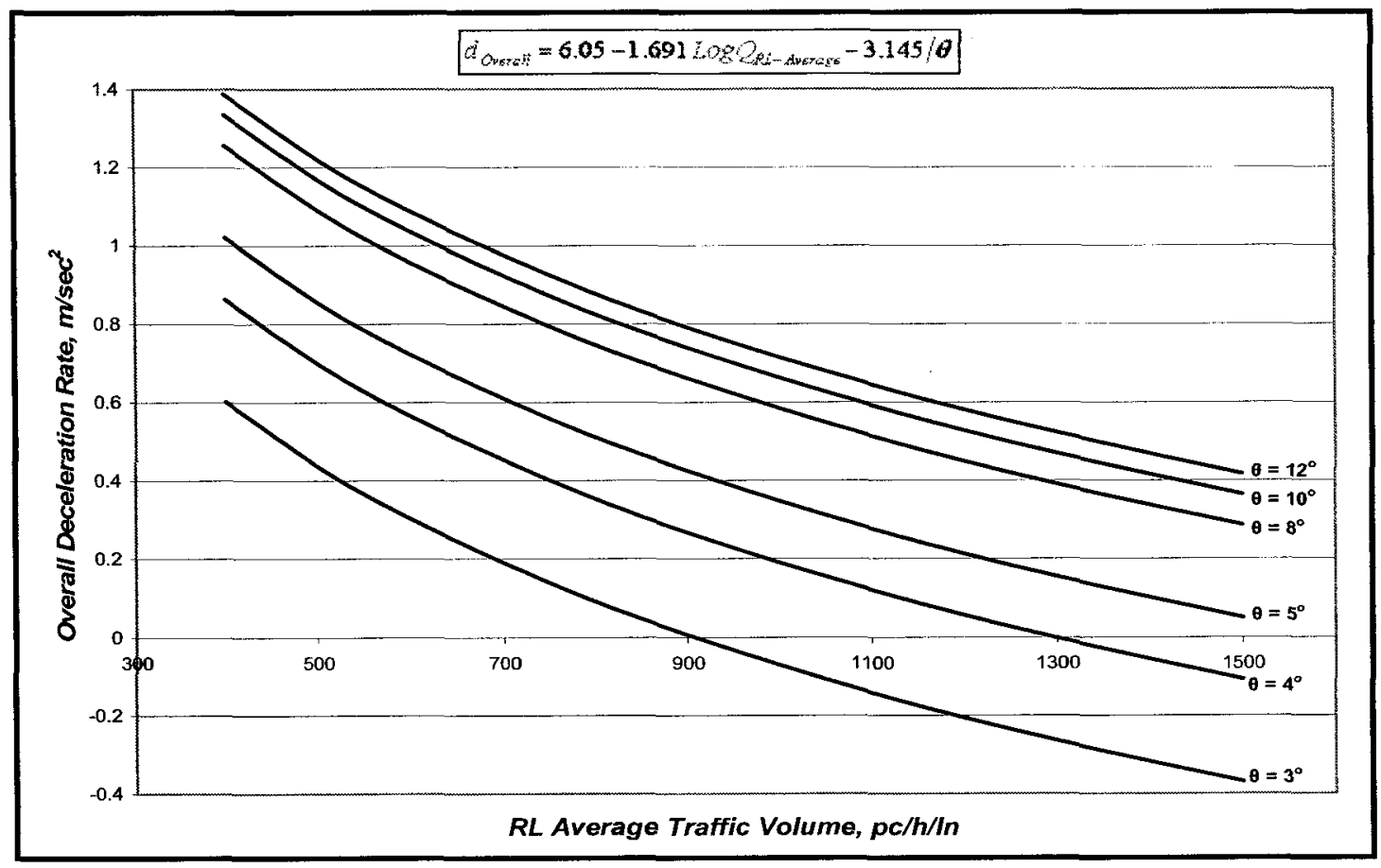

Figure 6.4: Application of Overall Deceleration Rate Based on RL Average Traffic Volume and Divergence Angle

As shown in Figure 6.4, the overall deceleration rate on SCLs is expected to increase with increase in the divergence angle and/or decrease in the RL average traffic volume along the freeway diverge area. For example, the overall deceleration rate on a particular deceleration SCL length is expected to be $1.021 \mathrm{~m} / \mathrm{sec}^{2}$ while designing a divergence angle of $5^{\circ}$ for a RL average traffic volume of $400 \mathrm{pc} / \mathrm{h} / \mathrm{ln}$ along the diverge area. The increase in the RL average traffic volume along the freeway diverge area from 400 to $1400 \mathrm{pc} / \mathrm{h} / \mathrm{ln}$ for the same divergence angle of $5^{\circ}$ would reduce the overall deceleration rate to $0.101 \mathrm{~m} / \mathrm{sec}^{2}$. On the other hand, as the divergence angle at the physical nose increases to be $10^{\circ}$ for the same traffic volume, the estimated overall deceleration rate would increase from 1.021 to be about $1.335 \mathrm{~m} / \mathrm{sec}^{2}$. As mentioned earlier, consistency between the upstream and downstream geometry of the gore area of 
the exit terminal is necessary to allow the diverging vehicles to operate effectively on freeway diverge areas.

\subsection{APPLICATION OF MAXIMUM DECELERATION RATE}

The application illustration of the selected model for estimating the maximum deceleration rate $\left(d_{M a x}\right)$ on deceleration SCLs resulted in creation of several graphs as potential design aids for highway designers and professional. The developed model is a successful tool to estimate the maximum deceleration rate on SCLs while knowing the $\mathrm{RL}$ average traffic volume ( $\left.Q_{R L \text {-Average }}\right)$, speed differential $\left(\Delta V_{R L-D i v e r g e}\right)$, and the SCL type (limited or extended). Figure 6.5 shows a series of graphs of the estimated maximum deceleration rate plotted against RL average traffic volume for different speed differentials between the freeway RL mean speed and the diverge speed as well as the SCL type. Four graphs were created for different average heavy vehicle percentages along the freeway diverge area of $6,10,14$, and $20 \%$.

As previously mentioned, the modelling attempts to develop predictive models capable of estimating the maximum deceleration rate on limited length type SCLs only were not successful and no models were developed. The maximum deceleration rate on limited length type SCLs was fairly constant among limited SCL sites selected in the study and took an average rate of $2.235 \mathrm{~m} / \mathrm{sec}^{2}$. Therefore, this maximum deceleration rate could be used as the maximum comfortable deceleration rate on limited length type SCLs. 


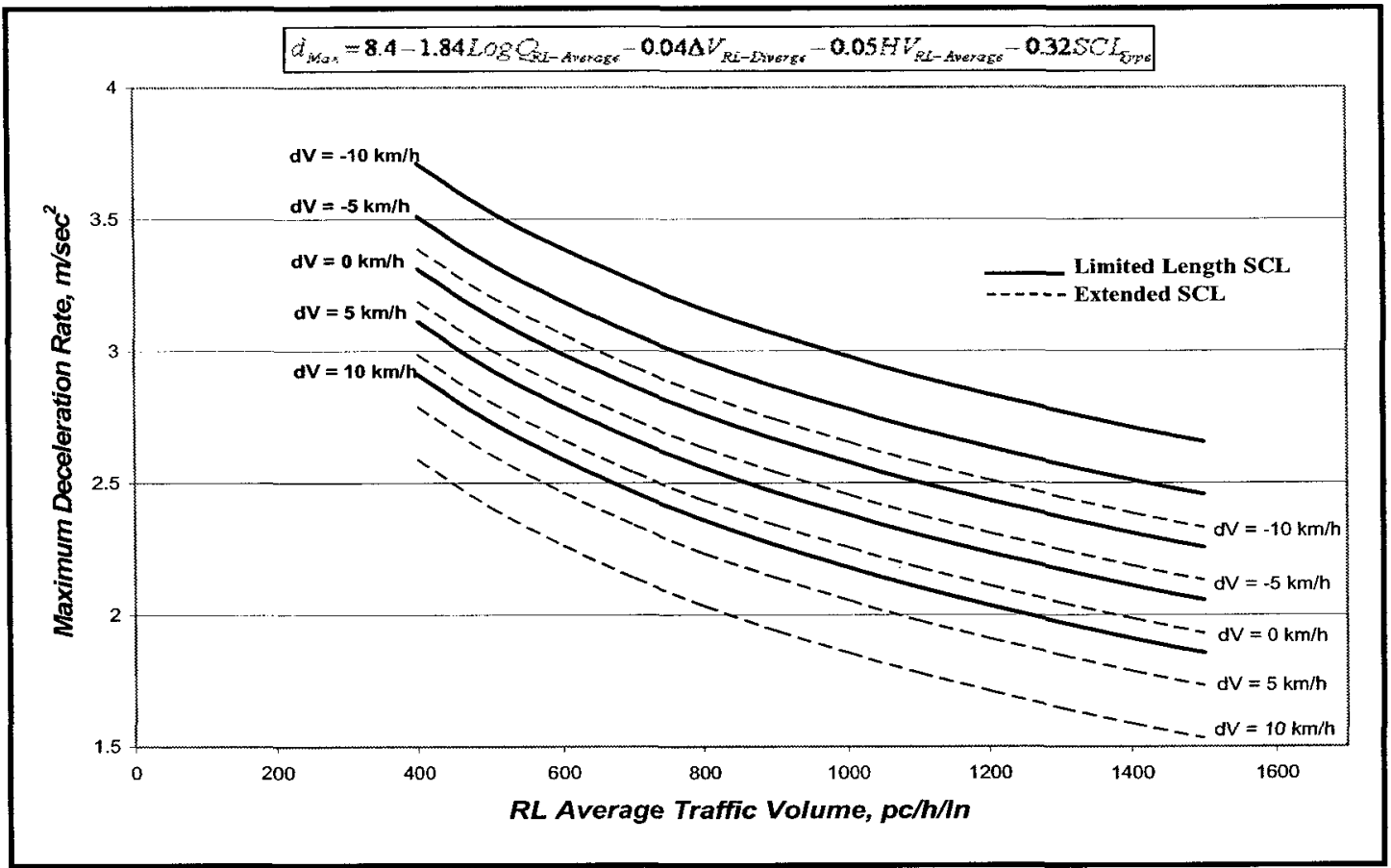

Figure 6.5.a: Application of Maximum Deceleration Rate Based on RL Average Traffic Volume, Speed Differential, and SCL Type $(\mathrm{HV} \%=6)$

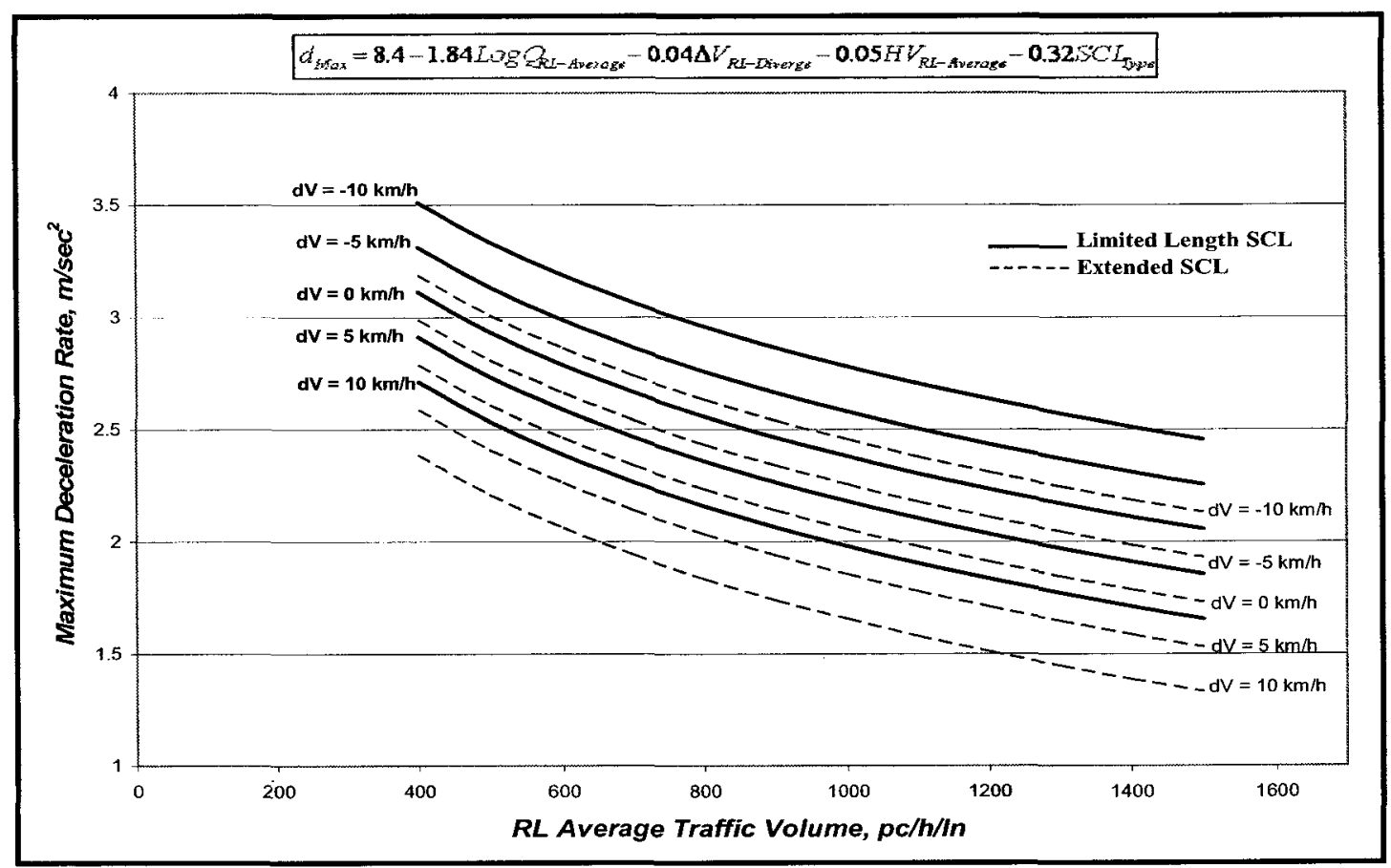

Figure 6.5.b: Application of Maximum Deceleration Rate Based on RL Average Traffic Volume, Speed Differential, and SCL Type $($ HV \% $=10)$ 


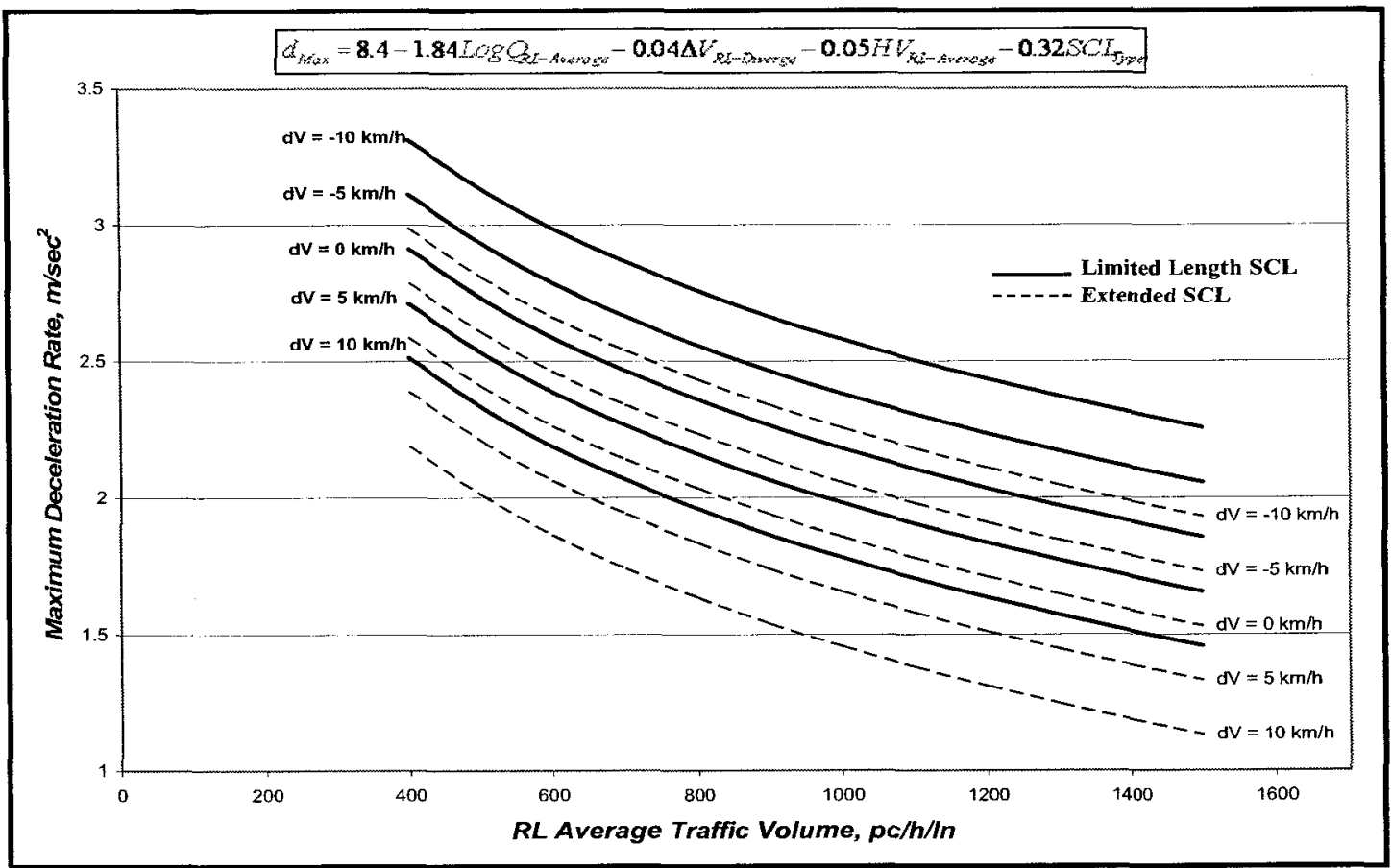

Figure 6.5.c: Application of Maximum Deceleration Rate Based on RL Average Traffic Volume, Speed Differential, and SCL Type $(H V \%=14)$

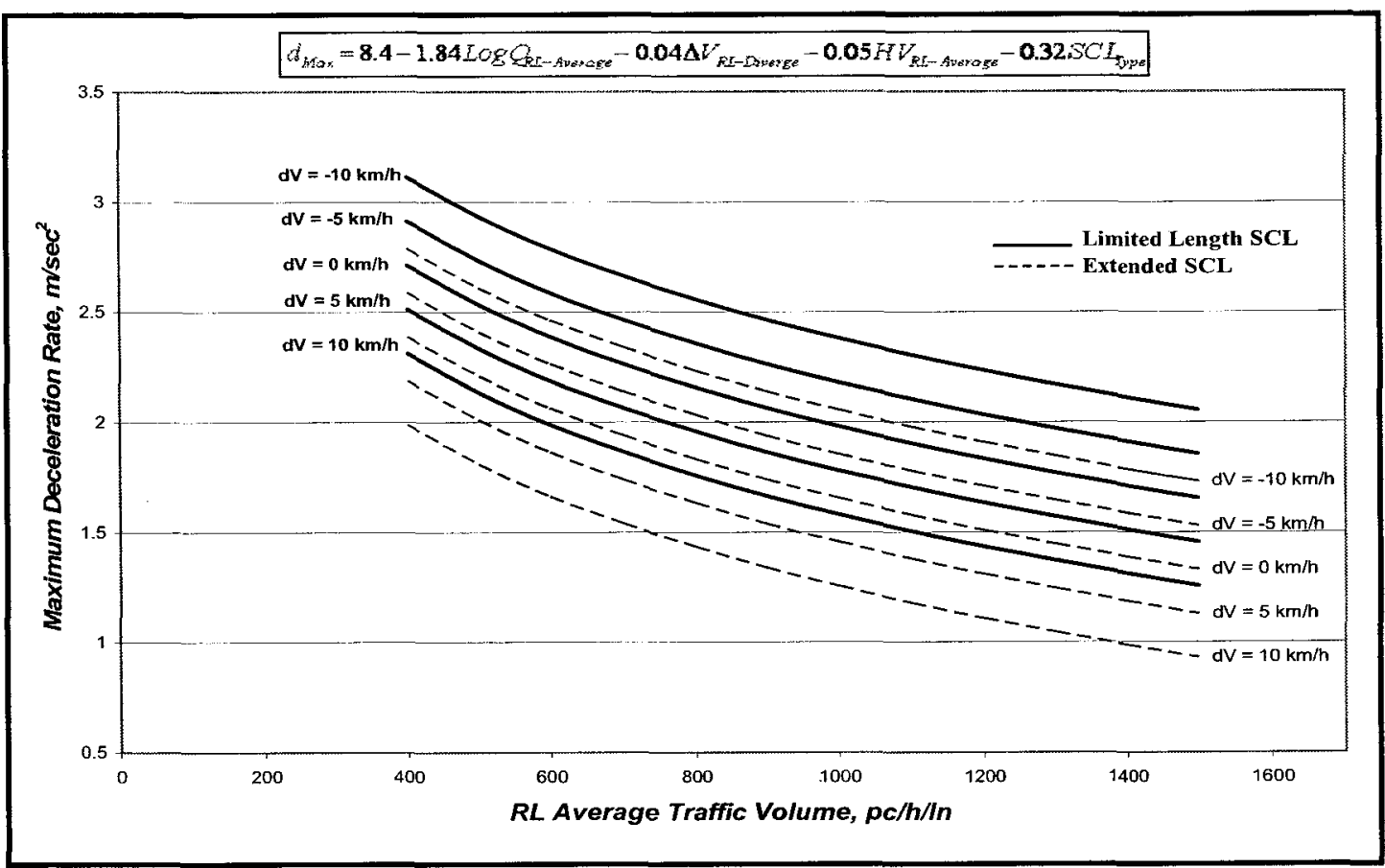

Figure 6.5.d: Application of Maximum Deceleration Rate Based on RL Average Traffic Volume, Speed Differential, and SCL Type $(H V \%=20)$ 
In Figures 6.5.a through 6.5.d, the highway designer could predict the maximum deceleration rate while knowing the RL average traffic volume and RL average HV \% along the freeway diverge area as well as the SCL type and a desired speed differential. As shown earlier in the speed differential model application (Section 5.7.2), the speed differential between he freeway RL mean speed and the diverge speed depend mainly on the geometry of the deceleration SCL and ramp of the exit terminal. Therefore, exiting vehicles that diverge with large speed disparities would endanger travelling vehicles on freeway diverge areas.

The application of the illustrations shown in Figure 6.5 would help designers to estimate the maximum deceleration rate on deceleration SCLs. For example, in Figure 6.5.c, the estimated maximum deceleration rate is expected to be $1.911 \mathrm{~m} / \mathrm{sec}^{2}$ for a limited SCL, RL average traffic volume of $1400 \mathrm{pc} / \mathrm{h} / \mathrm{ln}$, and RL average heavy vehicle percentage of $14 \%$ for a desired speed differential of $0.0 \mathrm{~km} / \mathrm{h}$. The estimated maximum deceleration rate would decrease to be $2.912 \mathrm{~m} / \mathrm{sec}^{2}$ as the RL average traffic volume along the diverge area decrease to $400 \mathrm{pc} / \mathrm{h} / \mathrm{ln}$ for the same type of SCL (limited), RL average $\mathrm{HV} \%$, and the desired speed differential. For the same RL average traffic volume and HV \% along the freeway diverge area and the same desired speed differential, increasing the length of the deceleration SCL would reduce the maximum deceleration rate. However, as speed differential increases the maximum deceleration rate is expected to decrease on the SCL. Nevertheless, the designer could use the maximum deceleration rate model to reduce the stress on drivers while performing deceleration behaviour on a particular deceleration SCL length. 


\subsection{APPLICATION OF EFFECTIVE DECELERATION DISTANCE}

The application illustration of the selected model for estimating the effective deceleration distance $\left(D_{D e c}\right)$ on deceleration SCLs related the SCL length $\left(L_{D e c}\right)$ and the RL average HV \% along the diverge area as shown in Figure 6.6. The model was plotted as a potential design aid for estimating the effective deceleration distance to be used by diverging vehicles from the available deceleration SCL length for different RL average HV \% along the freeway diverge area.

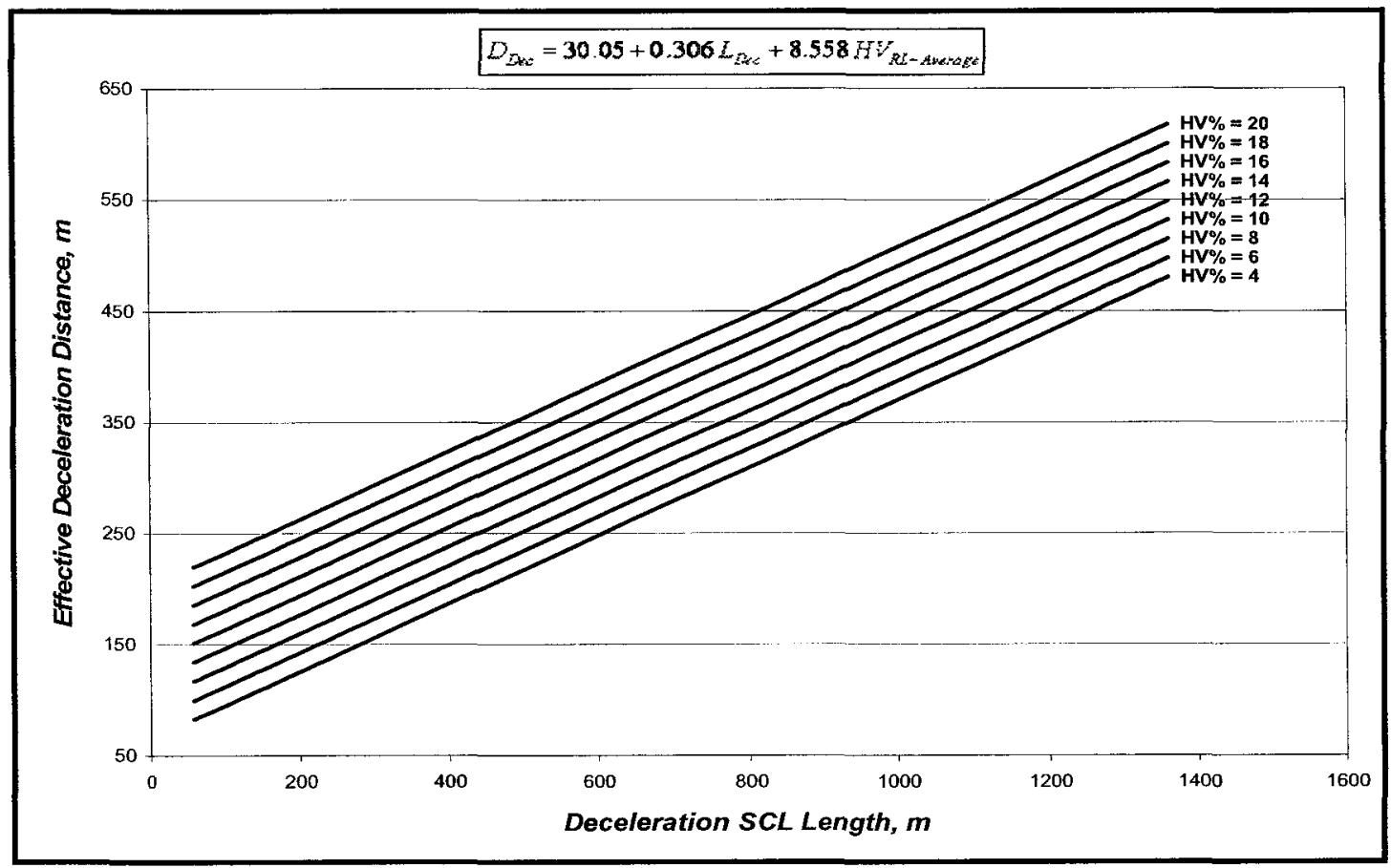

Figure 6.6: Application of Effective Deceleration Distance Based on SCL Length and Heavy Vehicles Percentage (HV\%)

In Figure 6.6, the highway designer could estimate the effective deceleration distance from a deceleration SCL of known length for different RL average $\mathrm{HV} \%$ along the freeway diverge area. For example, the effective deceleration distance is estimated to 
be of about $295.2 \mathrm{~m}$ on a deceleration SCL length of $475 \mathrm{~m}$ and RL average HV percentage of $14 \%$. As the length of the deceleration SCL increase to $1200 \mathrm{~m}$ (an extended length type SCL) and for the same percentage of HV on freeway right lane along the diverge area of $14 \%$, the estimated effective deceleration distance is expected to increase to $517.1 \mathrm{~m}$. On the other hand, the designer may estimate a length of deceleration SCL to be provided consequent to a desired effective deceleration distance for different RL average $\mathrm{HV} \%$ along the diverge area. This plotted model in Figure 6.6 is valid for limited and extended deceleration length type SCLs ranging from 58 to $1358 \mathrm{~m}$. 


\section{CHAPTER SEVEN \\ 7.0 CONCLUSIONS AND RECOMMENDATIONS}

\subsection{CONCLUSIONS}

Safe and efficient operations of freeway diverge areas are highly responsive to the geometry of exit terminal and traffic volumes on such designated areas. In this study, efforts were carried out to study the effect of geometric features and traffic conditions on operational diverging behaviour in freeway diverge areas. Hence, 13 diverge areas from Highway 417 in the City of Ottawa, Canada, were selected and used to collect speed data for 3,098 vehicles on both the freeway deceleration speed change lanes (SCL) and the adjacent right lanes (RL) during off-peak hour traffic conditions using two SpeedLaser ${ }^{\circledR}$ guns. The prevailing traffic on selected sites were recorded using a video camera that was mounted on a tripod and adjusted to record the traffic streams and vehicle movements on freeway diverge areas. The geometric data related to the selected study sites were gathered and several geometric elements were extracted for each site. Moreover, the collision data associated with selected sites were also collected to be part of the database prepared for the purpose of this study and specifically for safety performance purposes.

The driver behaviour at freeway diverge areas is generally affected by many factors. Hence, modelling driver behaviour on freeway diverge areas was the main objective of this research for capturing the variation in many aspects of diverging behaviour of exiting vehicles. The modelling attempts for developing models capable of predicting the driver behaviour at freeway diverge areas were successful and resulted in creation of 34 statistically significant predictive models at $5 \%$ level of significance through application of multiple linear regression analysis. The development of such 
statistically significant behavioural models was the most important conclusion of this study. The developed models related geometric features and traffic volumes to several driver behaviour measures. Operational performance models developed in the study for diverging vehicles comprehended the following driving behaviour parameters:

- Freeway RL mean speed along freeway diverge area $\left(V_{R L}\right)$

- Diverge speed onto SCL ( $\left.V_{\text {Diverge }}\right)$

- Speed differential between RL mean speed and diverge speed $\left(\Delta V_{R L-D i v e r g e}\right)$

- Gore speed at end of SCL $\left(V_{\text {Gore }}\right)$

- Overall deceleration rate on SCL $\left(d_{\text {Overall }}\right)$

- Maximum deceleration rate on $\operatorname{SCL}\left(d_{M a x}\right)$

- Effective deceleration distance used from diverge point to end of SCL ( $\left.D_{D e c}\right)$

Unfortunately, the modelling attempts for creating safety performance modes to predict collision frequencies on freeway diverge areas were not successful and no models were developed. Nevertheless, the developed models would be of assistance to highway designers and professionals as well as decision makers in selecting the proper geometry of exit terminals (particularly the deceleration SCL length) for better operations and safety on freeway diverge areas. The main conclusions resulting from the regression analyses could be summarized as follows:

- The targeted heavy vehicles on selected freeway deceleration SCLs and the adjacent RLs were found not to have any significant effect on the aggregated driver behaviour measures. Consequently, modelling attempts in this study considered passenger cars only. 
- Freeway RL mean speed, determined as the population's $85^{\text {th }}$ percentile value of vehicle mean speed, along the diverge area ranged from $99.4 \mathrm{~km} / \mathrm{h}$ to 117.9 $\mathrm{km} / \mathrm{h}$ for all vehicle movements (through vehicles, merged left vehicles onto a freeway inner lane, and diverged vehicles onto SCL). Freeway RL mean speed was found to be influenced by deceleration SCL length and RL traffic volume downstream the freeway diverge area for limited length type SCLs only. Two predictive models were developed for estimating the freeway RL mean speed along limited length type SCLs.

- Diverge speed, determined as the population's $85^{\text {th }}$ percentile value of vehicle diverge speed, on deceleration SCL ranged from $80.4 \mathrm{~km} / \mathrm{h}$ to $120.1 \mathrm{~km} / \mathrm{h}$ among the study sites. Diverge speed was observed to highly depend on the freeway RL mean speed, deceleration SCL length, divergence angle at the physical nose, and diverging traffic. Nine models were developed for predicting the diverge speed on deceleration SCLs. There were four models associated with limited length type SCLs and five models were capable of estimating diverge speed on both limited and extended length type SCLs.

- Speed differentials between $85^{\text {th }}$ percentile RL mean speeds and $85^{\text {th }}$ percentile diverge speeds ranged between $-8.2 \mathrm{~km} / \mathrm{h}$ and $16.1 \mathrm{~km} / \mathrm{h}$ among the study sites. The speed differential was shown to be influenced by the deceleration SCL length, transition length, RL traffic volume upstream the freeway diverge area, and the traffic composition. The modelling attempts in this study succeeded in developing seven significant predictive models capable of estimating the speed differential on freeway diverge areas. Two 
models were related to limited length type SCLs while the other five models were useful for both deceleration SCL types.

- The gore speed, determined as the population's $85^{\text {th }}$ percentile value of vehicle gore speed, at end of SCL (1.25 m marking separation) was observed to range from $78.7 \mathrm{~km} / \mathrm{h}$ to $109.4 \mathrm{~km} / \mathrm{h}$ among the study sites. The gore speed was shown to be influenced by the diverge speed, deceleration SCL length, transition length, divergence angle, and diverging traffic. Six predictive models were developed for estimating the gore speed with four models related to limited length type SCLs and two models related to combined (limited and extended) SCLs.

- The overall deceleration rate, determined as the population's $85^{\text {th }}$ percentile value of vehicle overall deceleration rate, on selected SCLs ranged from 0.351 $\mathrm{m} / \mathrm{sec}^{2}$ to $1.375 \mathrm{~m} / \mathrm{sec}^{2}$. The overall deceleration rate was shown to be influenced by the freeway geometry and freeway RL traffic volume along the diverge area. Seven significant models were developed for predicting the overall deceleration rate on SCLs. There was one model associated with limited SCL length type and the six other models were practical for combined SCLs.

- The maximum deceleration rate, determined as the population's $85^{\text {th }}$ percentile value of vehicle maximum deceleration rate, on SCLs ranged from $1.688 \mathrm{~m} / \mathrm{sec}^{2}$ to $2.815 \mathrm{~m} / \mathrm{sec}^{2}$ among study sites. The maximum deceleration rate was shown to be influenced by the freeway $R L$ traffic volume along the diverge area, traffic composition, and the speed differential between RL mean 
speed and diverge speed. No models were develop for estimating the maximum deceleration rate on limited SCLs as the $85^{\text {th }}$ percentile maximum deceleration rates on limited length type SCLs in this study were fairly constant. This indicated a maximum comfortable deceleration rate of 2.235 $\mathrm{m} / \mathrm{sec}^{2}$ on limited SCLs. However, one model was developed for estimating the maximum deceleration rate for combined SCLs.

- The effective deceleration distance determined as the population's $85^{\text {th }}$ percentile value of vehicle effective deceleration distance for all selected sites and measured from the diverge point to the end of the SCL ranged from 41.8 $\mathrm{m}$ to $448.7 \mathrm{~m}$. The effective deceleration distance determined as the population's $95^{\text {th }}$ percentile value of vehicle effective deceleration distance ranged from $48.1 \mathrm{~m}$ to $514.3 \mathrm{~m}$ among study sites. The effective deceleration distance was found to be influenced by the SCL length to be provided as well as the percentage of $\mathrm{HV}$ on freeway right lane along the relevant SCL. Two models were developed for estimating the effective deceleration distance in this study with one model for limited length type SCLs and other model for combined SCLs.

- A length of $475 \mathrm{~m}$ for limited length type SCLs was shown to be adequate for completing the deceleration behaviour in a comfortable and safe manner, serving $95 \%$ of the population.

- The models developed in this study are considered an important achievement and have provided promising results concerning driver diverging behaviour, in 
contribution to the limited research related to evaluation of freeway diverge areas.

\subsection{RECOMMENDATIONS FOR FUTURE RESEARCH}

Based on the results and findings of this study, the following are recommended as future work in the area of evaluating freeway diverge areas:

- The use of more data and increasing the number of sites would provide more strength to the developed models and improve their prediction capabilities.

- Validation of the developed models using wider range of data from different freeway diverge areas at different freeways and interchanges would enhance the models' reliability.

- Collecting speed data on exit ramp curves and inclusion of ramp radius with a wide range of curvatures would be cooperative in evaluating the driver behaviour downstream the gore area of exit terminals.

- Collecting speed data for heavy vehicles and modelling for their behaviour would help capture the differences in diverging behaviour of different types of exiting vehicles at freeway diverge areas.

- Intended for a wider scope of research, criteria to evaluate the driver diverging behaviour in terms of the differences between the operational speed and design speed on freeway diverge areas should be introduced in North American guidelines, if possible. 


\section{REFERENCES}

AASHTO (2001) "A Policy on Geometric Design of Highways and Streets", American Association of state Highway and Transportation Officials, Washington, D.C.

Ahammed, M. A. (2005) "Freeway Merging Behavior and Safety of Acceleration Lanes: Field Study", M.A.Sc. Thesis - Department of Civil and Environmental Engineering, Carleton University, Ottawa, Ontario, Canada.

Al-Kaisy, A., Stewart, J. A., and Van Aerde M. (1999) “A simulation approach for examining capacity and operational performance at freeway diverge areas", Canadian Journal of Civil Engineering, Vol. 26 (6): 760-770.

Al-Masaeid, H. R., Hamed, M., Aboul-Ela, M. T., and Ghannam, A. G. (1995) "Consistency of Horizontal Alignment for Different Vehicle Classes", Transportation Research Record 1500: 178-183.

Anderson, I. B, Bauer K. M., Harwood D. W., and Fitzpatrick K. (1999) "Relationship to Safety of Geometric Design Consistency Measures for Rural Two-Lane Highways", Transportation Research Record 1658: 43-51.

Awad, W. H., and Janson B. N. (1998) "Prediction Models for Truck Accidents at Freeway Ramps in Washington State Using Regression and Intelligence Techniques", Transportation Research Record 1635: 30-36.

Awatta, M. (2003) "Highway Design Consistency and Safety: Individual and Overall Evaluation Criteria”, M.A.Sc. Thesis - Department of Civil and Environmental Engineering, Carleton University, Ottawa, Ontario, Canada. 
Baker, M. Jr. (1980) "Improving the Traffic Operations and Safety of Ramps and Speed Change Lanes - A Review of State Design Practices", Federal Highway Administration, Washington D.C. USA. Report No. FHWA-RD-79-127, Interim Report-September: 1-43.

Bared, J., Giering, G. L., and Warren, D. L. (1999) "Safety Evaluation of Acceleration and Deceleration Lane Lengths", ITE Journal - Institute of Transportation Engineers, Vol. 69 (5): 50-54.

Batenhorst, R. A., and Gerken, J. G. (2000) "Operational Analysis of Terminating Freeway Auxiliary Lanes with One-Lane and Two-Lane Exit Ramps: A Case Study", Proceeding of Mid-Continent Transportation Symposium: 72-76.

Bauer, K.M., and Harwood, D. W. (199'7) "Statistical Models of Accidents on Interchange Ramps and Speed-Change Lanes”, Federal Highway Administration, Washington, D.C., Report No. FHWA-RD-97-106.

Bennett, C. R., and Dunn, R.C. M. (1995) "Driver Deceleration Behaviour on a Freeway in New Zealand”, Transportation Research Record 1510: 70-83.

Berry, D. S., Ross, G. L., and Pfefer, R. C. (1963) “A Study of Left-Hand Exit Ramps on Freeways", Highway Research Board, Highway Research Record 21: 1-16.

Brown, B., and Baass, K. (1997) "Seasonal Variation in Frequencies and Rates of Highway Accidents as Function of Severity", Transportation Research Record 1581: 59-65.

Cirillo, J.A. (1970) "The Relationship of Accidents to Length of Speed-Change Lanes and Weaving Areas on Interstate Highways", Highway Research Board, Highway Research Record 312: 17-32. 
Conklin, R. D. (1959) “A Comparison of Vehicle Operating Characteristics between Parallel Lane and Direct Taper Types of Freeway Off-Ramps", Traffic Engineering, Vol. 30 (3): 13-17.

Davis, M. M., and Williams, K. M. (1968) "Vehicle Operating Characteristics on outer Loop Deceleration Lanes of Interchanges", Department of Civil Engineering, University of Toronto. In corporation with the Ontario Department of Highways, Ontario Joint Highway Research Programme, Report No. 43.

Dixon, K. K., Wu, C. H., Sarasua, W., and Daniel J. (1999) "Estimating Free-Flow Speeds for Rural Multilane Highways", Transportation Research Record 1678: 73-82.

Dutta, A., Carpenter R., Noyce D. A., Duffy S. A., and Fisher, D. L. (2002) 'Drivers' Understanding of Overhead Freeway Exit Guide Signs: Evaluating for Alternatives with an Advanced Fixed-Base Driving Simulator", Transportation Research Record 1803: $102-109.8$

Fazio, J., Michaels, R. M., Reilly, W. R., Schoen, J., and Poulis, A. (1990) "Behavioral Model of Freeway Exiting", Transportation Research Record 1281: 16-27.

Fisher, R. L. (1948) "Traffic Performance on Acceleration and Deceleration Lanes", Abstracts, Highway Research Board, Vol. 18 (4).

Fukutome, I., and Moskowitz, K. (1963) "Traffic Behaviour and Off-Ramp Design", Highway Research Board, Highway Research Record 21: 17-32.

Garber, N. J. and Hoel, L. A. (2002) "Traffic and Highway Engineering”, Third Edition, Thomson Learning Inc., California, USA. 
Garcia, A. and Romero, M. A. (2005) "Tracking Vehicle Behavior in a Deceleration Lane with Different Lengths", Transportation Research Board, 3rd International Symposium on Highway Geometric Design, Chicago, Illinois: 1-17.

Golob, T. F., and Recker, W. W. (2003) "Relationships Among Urban Freeway Accidents, Traffic, Flow, Weather, and Lighting Conditions", Journal of Transportation Engineering 129 (4): 342-353.

Golob, T. F., Recker, W. W., and Alvarez, V. M. (2004) "Safety Aspects of Freeway Weaving Sections", Transportation Research. Elsevier Ltd. Part A (38): 35-51.

Grafen, A. and Hails, R. (2002) "Modern Statistics for the Life Sciences", Oxford University Press Inc., New York, USA.

Harwood, D. W. (1995) "Relationships Between Operational and Safety Considerations in Geometric Design Improvements", Transportation Research Record 1512: 1-6.

Harwood, D. W., and Mason J. M. Jr. (1993) “Ramp/Mainline Speed Relationships and Design Considerations", Transportation Research Record 1385: 121-125.

Hauer, E. (2001) "Computing and Interpreting Accident Rates for Vehicle Types or Driver Groups", Transportation Research Record 1746: 69-73.

HCM (2000) "Highway Capacity Manual", Transportation Research Board, National Research Council. Washington, D.C.

Hunter, M., Machemehl, R., and Tsyganov, A. (2001) "Operational Evaluation of Freeway Ramp Design", Transportation Research Record 1751: 90-100.

Hutabarat, R. S. (2004) "Remedies for Driver Error", Arizona Department of Transportation, In Cooperation with U.S. Department of Transportation, Federal Highway Administration, Final Report 567. 
Janson, B. N., Awad, W., Robles, J., Kononov, J., and Pikerton, B. (1998) "Truck Accidents at Freeway Ramps: Data Analysis and High-Risk Site Identifications", Journal of Transportation and Statistics, Bureau of Transportation Statistics, US Department of Transportation, Vol. 1 (1):77-82.

Khan, S., Shanmugam, R., and Hoeschen, B. (1999) "Injury, Fatal, and Property Damage Accident Models for Highway Corridors", Transportation Research Record, 1665: 84-92.

Khorashadi, A. (1998) "Effect of Ramp Type and Geometry on Accidents", Callfornia Department of transportation, Federal Highway Administration, Washington, D.C., Report No. FHWA/CA/TE-98/13.

Kleinbaum, D. G. and Kupper, L. L. (1978) "Applied Regression Analysis and Other Multivariable Methods", Duxbury Press, California, USA.

Kockelman, K. M., and Ma, J. (2004) "Freeway Speeds and Speed Variation Preceding Crashes, Within and Across Lanes", Department of Civil Engineering, University of Texas, Austin, Annual Meeting of the Transportation Research Board: 1-19.

Koepke, F. J. (1993) "Ramp Exit/Entrance Design - Taper Verses Parallel and Critical Dimensions", Transportation Research Record 1385: 126-132.

ORSAR (2004) "Ontario Road Safety Annual Report”, Ontario Ministry of Transportation, Road Safety Program Office, Toronto, Ontario, Canada.

Lamm, R., Psarianos, B., Choueiri, E. A., and Mailaender, T. (1993) "Interchange Planning and Design - An International Perspective", Transportation Research Record 1385: 69-83. 
Lamm, R., Psarianos, B., and Mailaender, T. (1999) “Highway Design and Traffic Safety Engineering Handbook”, McGraw-Hill Companies, New York, USA.

Leisch, J. P. (1993) "Freeway and Interchange Design: A Historical Perspective", Transportation Research Record 1385: 60-68.

Leisch, J. P. (1993) "Operational Considerations for Systems interchanges", Transportation Research Record 1385: 106-111.

Leisch, J. P. (1998) “Comparison of Worldwide Practice in Interchange Design, International Symposium on Highway Geometric Design Practices, Boston, Massachusetts, Transportation Research Circular E - C003 (27): 1-9.

Liang, W. L., Kyte, M., Kitchener, F., and Shannon, P. (1998) “Effect of Environmental Factors on Driver Speed", Transportation Research Record 1635: 155-161.

Liapis, E. D., Psarianos, B., and Kasapi, E. (2001) "Speed Behaviour Analysis at Curved Ramp Sections of Minor Interchanges”, Transportation Research Record, 1751: 35-43.

Livneh, M., Polus, A., and Factor, J. (1988) "Vehicle Behaviour on Deceleration Lanes" Journal of Transportation Engineering, American Society of Civil Engineers (ASCE), 114 (6): 706-717.

Lum, H., and Reagan J. A. (1995) "Interactive Highway Safety Design Model: Accident Predictive Module", Public Roads, 58 (3).

Lundy, R.A. (1965) "The Effect of Ramp Type and Geometry on Accidents", Highway Research Record, Washington D.C., Report HRR 163

Lunenfeld, H. (1993) "Human Factors Associated with Interchange Design Features", Transportation Research Record 1385: 84-89. 
Martin, D. B., Newman, L., and Johnson, R. R. (1973) "Evaluation of Freeway Traffic Flow at Ramps, Collector Roads, and Lane Drops", Highway Research Record 432: $25-31$.

Maze, T. H., Preston, H., Storm, R. J., Neal, H., and Burchett, G. (2005) "Safety Performance of Divided Expressways, ITE Journal - Institute of Transportation Engineers, Vol. 75 (5): 48-53.

McCartt, A. T., Northrup, V. S., and Retting, R. A. (2004) “Types and Characteristics of Ramp Related Motor Vehicle Crashes on Urban Interstate Roadways in Northern Virginia”, Journal of Safety Research, Vol. 35: 107-114.

Mensah, A., and Hauer, E. (1998) “Two Problems of Averaging Arising in the Estimation of the Relationship between Accidents and Traffic Flow", Transportation Research Record 1635: 37-43.

Messer, C. J. (1980) "Methodology for Evaluating Geometric Design Consistency", Transportation Research Record 757: 7-14.

Miller, J.V. Jr. (1951) “A Deceleration Lane Study”, Traffic Engineering, Vol. 21 (11): $17-26$.

Ng, J., and Sayed, T. (2004) "Effect of Geometric Design Consistency on Road Safety", Canadian Journal of Civil Engineering, Vol. 31 (2): 218-227.

Ni, D., and Leonard, J. D. II. (2003) "Simulation of Freeway Merging and Diverging Behaviour", Proceeding of the 2003 Winter Simulation Conference: 1693-1700.

Pahl J. (1972) "Lane-Change Frequencies in Freeway Traffic Flow", Highway Research Record, Highway Research Board, Vol. 409: 17-25. 
Park, K. S., Lee, A. J., and Koh, B. K. (2001) "Drivers' Characteristics in the Perception of a Lead Vehicle's Deceleration Level", International Journal of Cognitive Ergonomics, 5 (2): 125-136.

Pedhazur, E. J. (1997) "Multiple Regression in Behavioral Research: Explanation and Prediction", Third Edition, Thomson Learning Inc., USA.

Persuad, B. and Dzbik, L. (1993) "Accident Prediction Models for Freeways", Transportation Research Record 1401: 55-60.

Retting, R. A., McGee, H. W., and Farmer, C. M. (2000) "Influence of Experimental Pavement Markings on Urban Freeway Exit-Ramp Traffic Speeds", Transportation Research Record 1705: 116-121.

Sarhan, M. E. A. (2004) "Safety Performance of Freeway Merge and Diverge Areas", M.A.Sc. Thesis - Department of Civil and Environmental Engineering, Carleton University, Ottawa, Ontario, Canada.

Sleet, D. A., and Branche, C. M. (2004) "Traffic Safety Is No Accident", Journal of Safety Research, Vol. 35: 173-174.

TAC (1999) "Geometric Design Guide for Canadian Roads", Transportation Association of Canada, Ottawa, Ontario, Canada.

Transport Canada (2004) "Canadian Motor Vehicle Traffic Collision Statistics", Road Safety and Motor Vehicle Regulation, Ottawa. Ontario, Canada.

Twomey, J. M., Heckman, M. L., Hayward, J. C., and Zuk, R. J. (1993) "Accidents and Safety Associated with Interchanges", Transportation Research Record 1385: 100-105. 
Twomey, J. M., Heckman, M. L., and Hayward, J. C. (1991) "Safety Effectiveness of Highway Design Features: Volume IV: Interchanges", Federal Highway Administration, Report No. FHWA-RD-91-047.

Walpole, R. E., Myers, R. H., Myers, S. L., and Ye, K. (2002) "Probability and Statistics for Engineers and Scientists", Seventh Edition, Prentice Hall Inc., New Jersey, USA.

Wortman, R. H. and Fox, T. C. (1994) “An Evaluation of Vehicle Deceleration Profiles”, Journal of Advanced Transportation, Vol. 28 (3): 203-215.

Zhou, M., and Sisiopiku, V. P. (1997) "Relationship between Volume-To-Capacity Ratios and Accident Rates", Transportation Research Record 1581: 47-52. 


\section{APPENDIX A: VEHICLE MOVEMENT DISTRIBUTION ON SELECTED STUDY SITES}




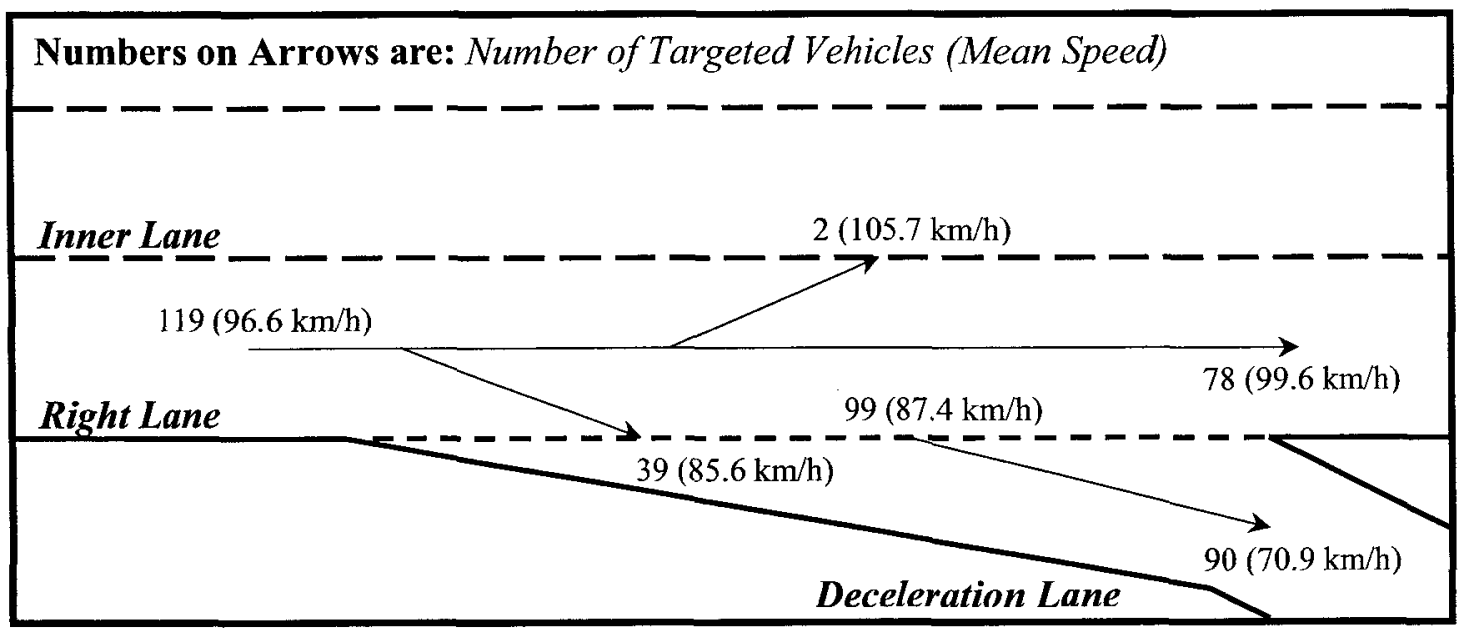

Figure A-1: Bronson Ave (W-NS) - Taper

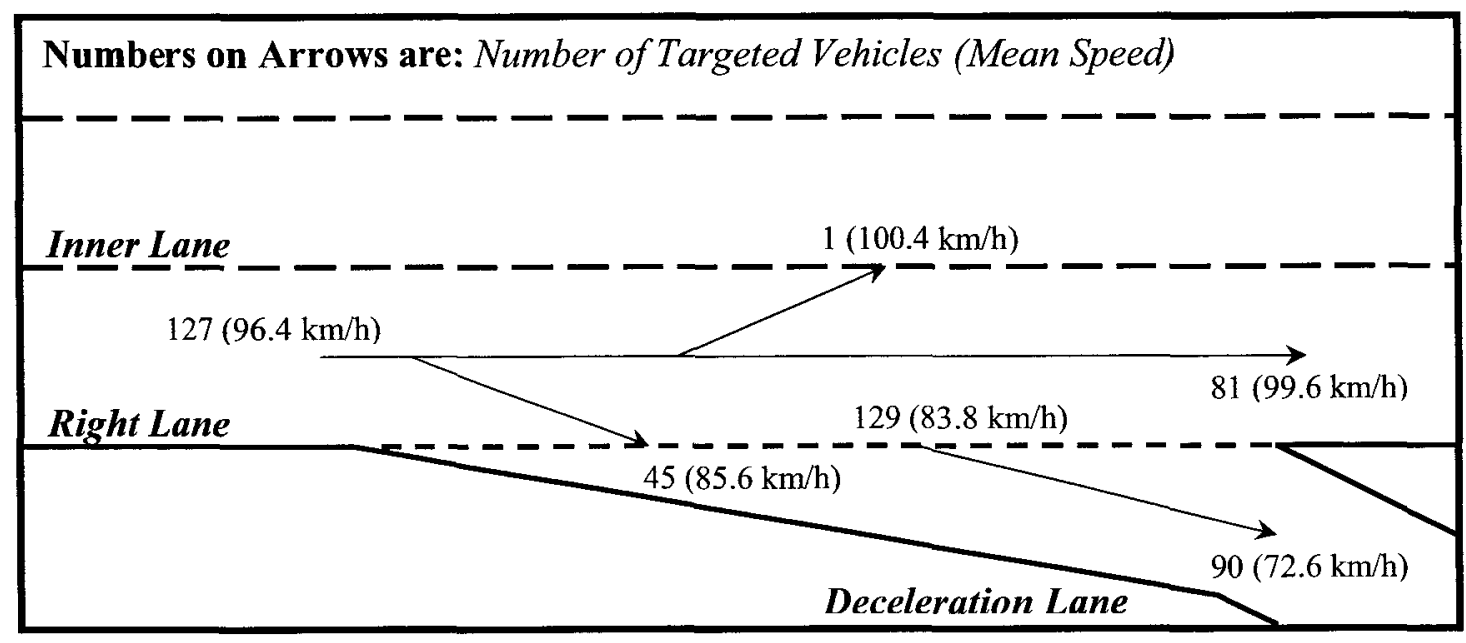

Figure A-2: Parkdale Ave (W-NS) - Taper 


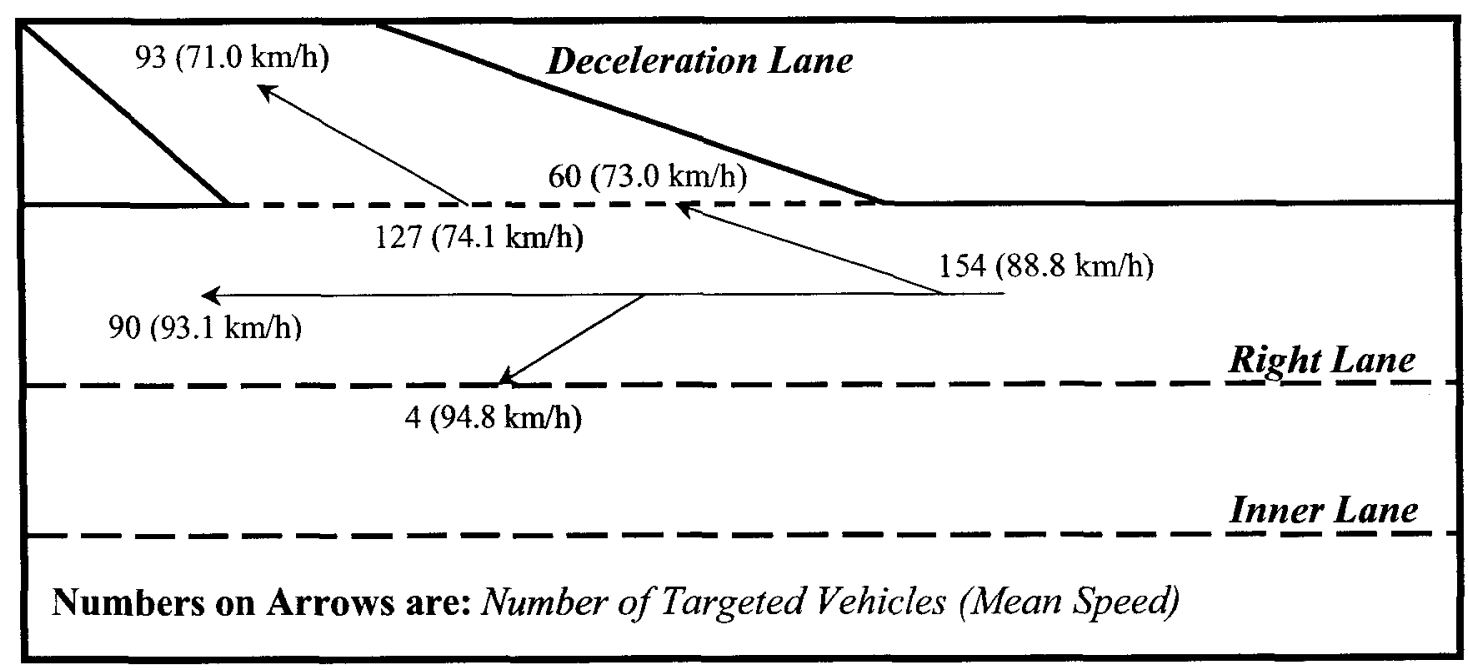

Figure A-3: Prom Island Park Dr (E-N) - Taper

Numbers on Arrows are: Number of Targeted Vehicles (Mean Speed)

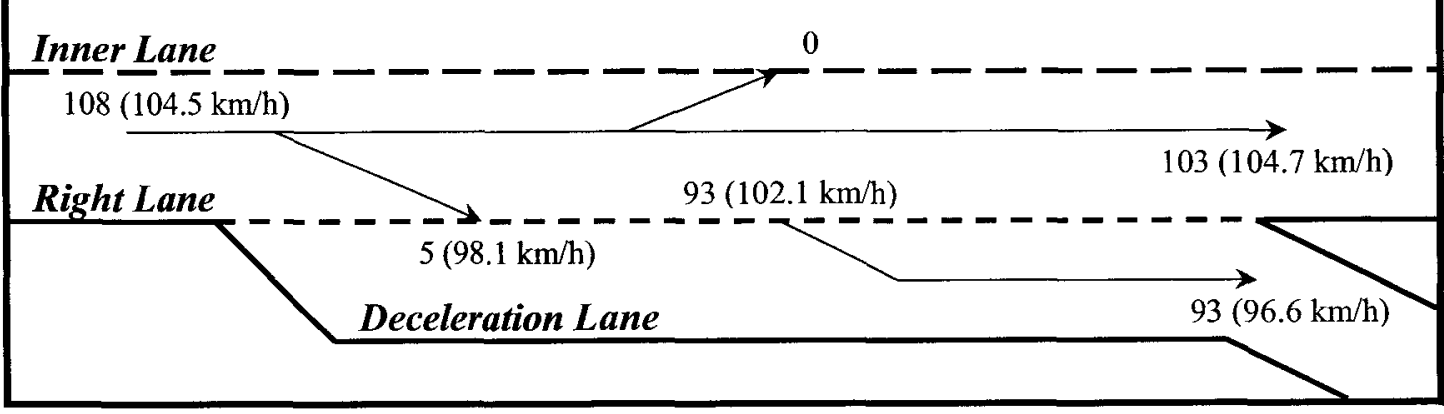

Figure A-4: Moodie Dr (W-NS) - Parallel 


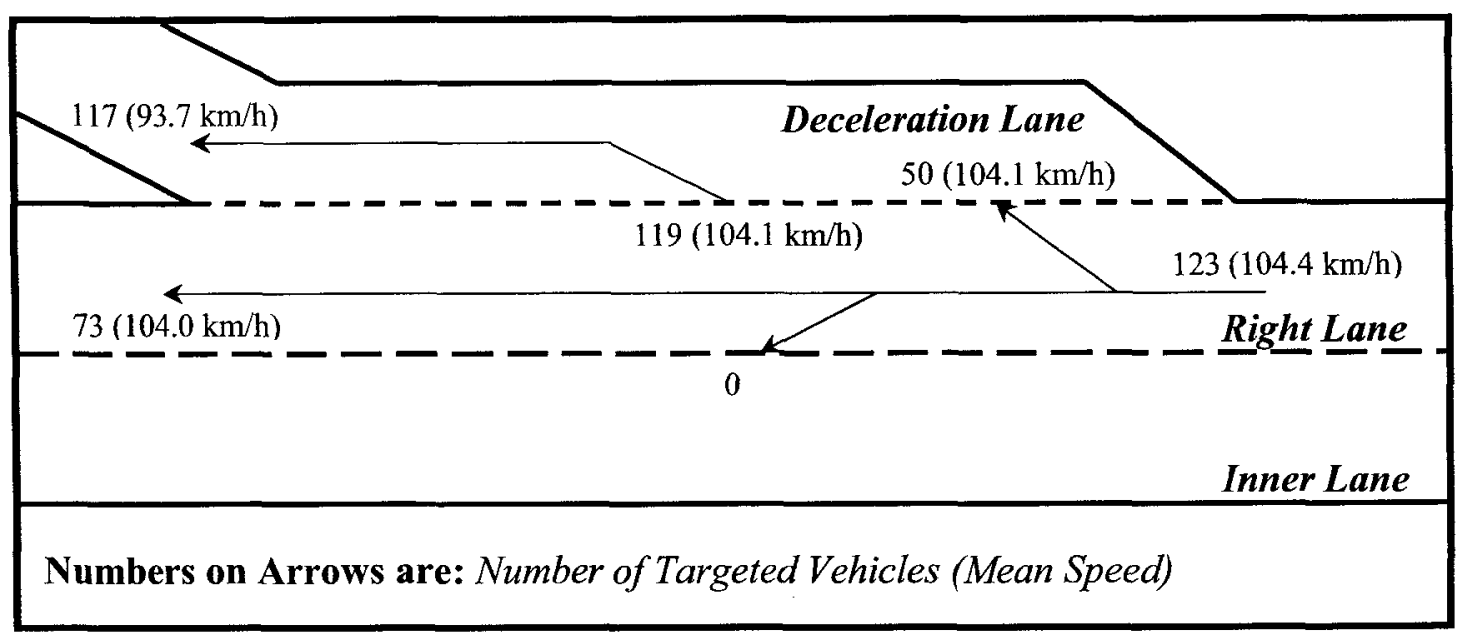

Figure A-5: Terry Fox Dr (E-NS) - Parallel

Numbers on Arrows are: Number of Targeted Vehicles (Mean Speed)

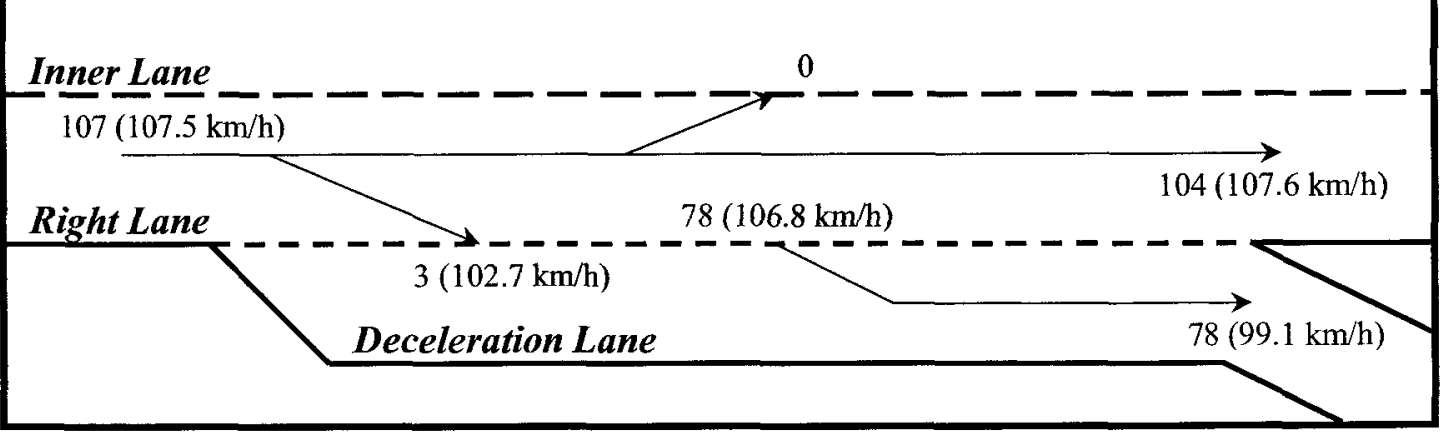

Figure A-6: Terry Fox Dr (W-NS) - Parallel 


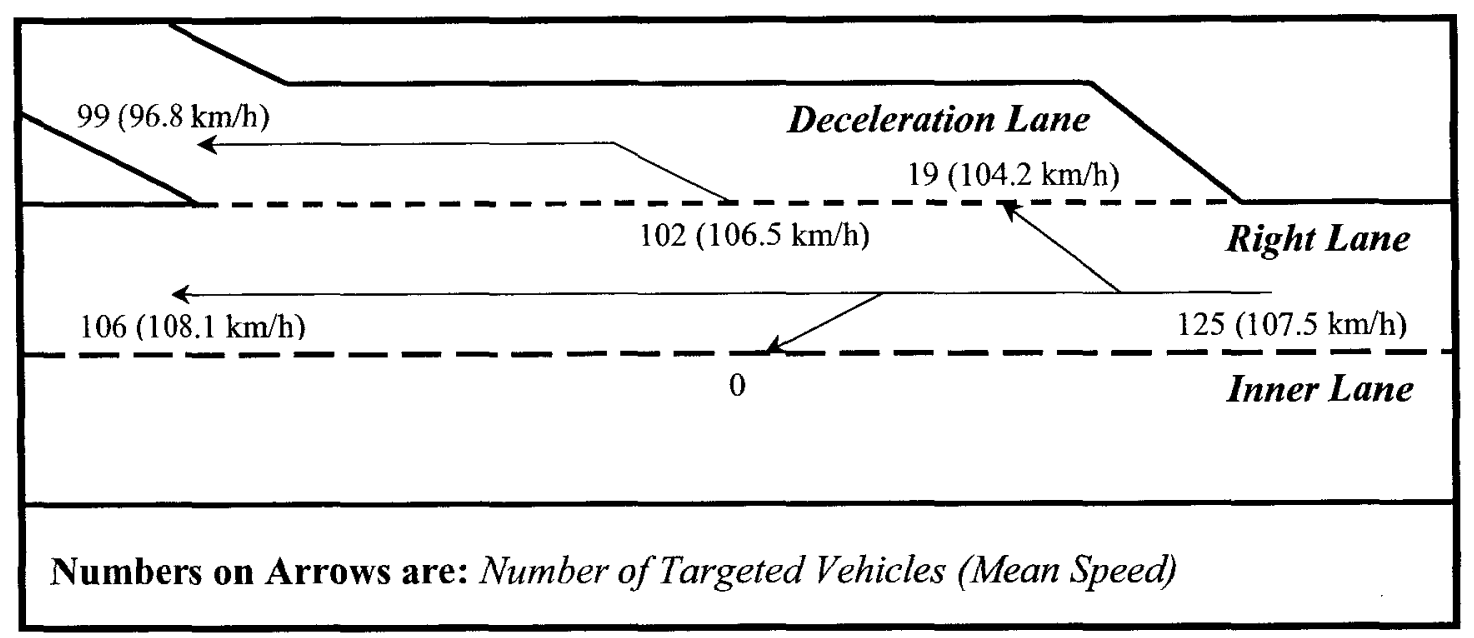

Figure A-7: Ch. Carp Rd (E-NS) - Parallel

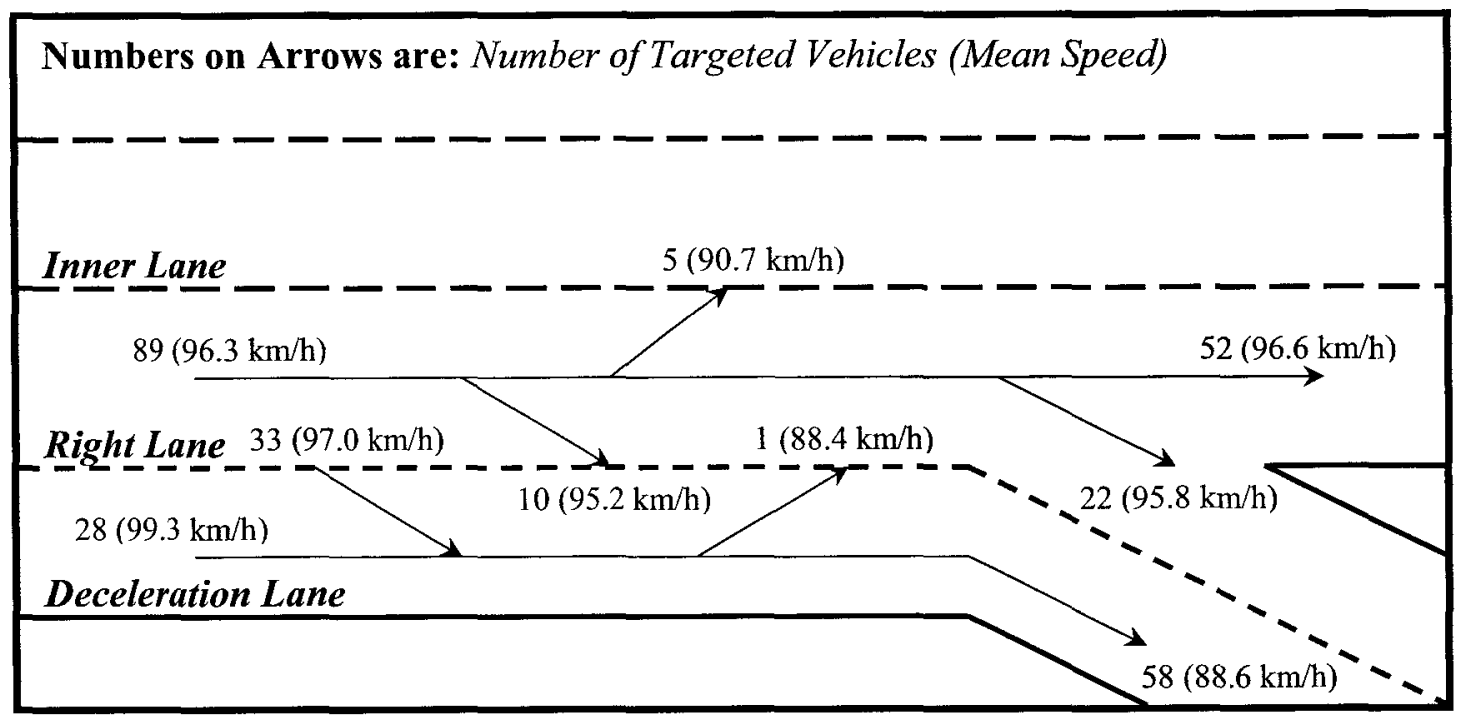

Figure A-8: St. Laurent Blvd (W-NS) - Extended 


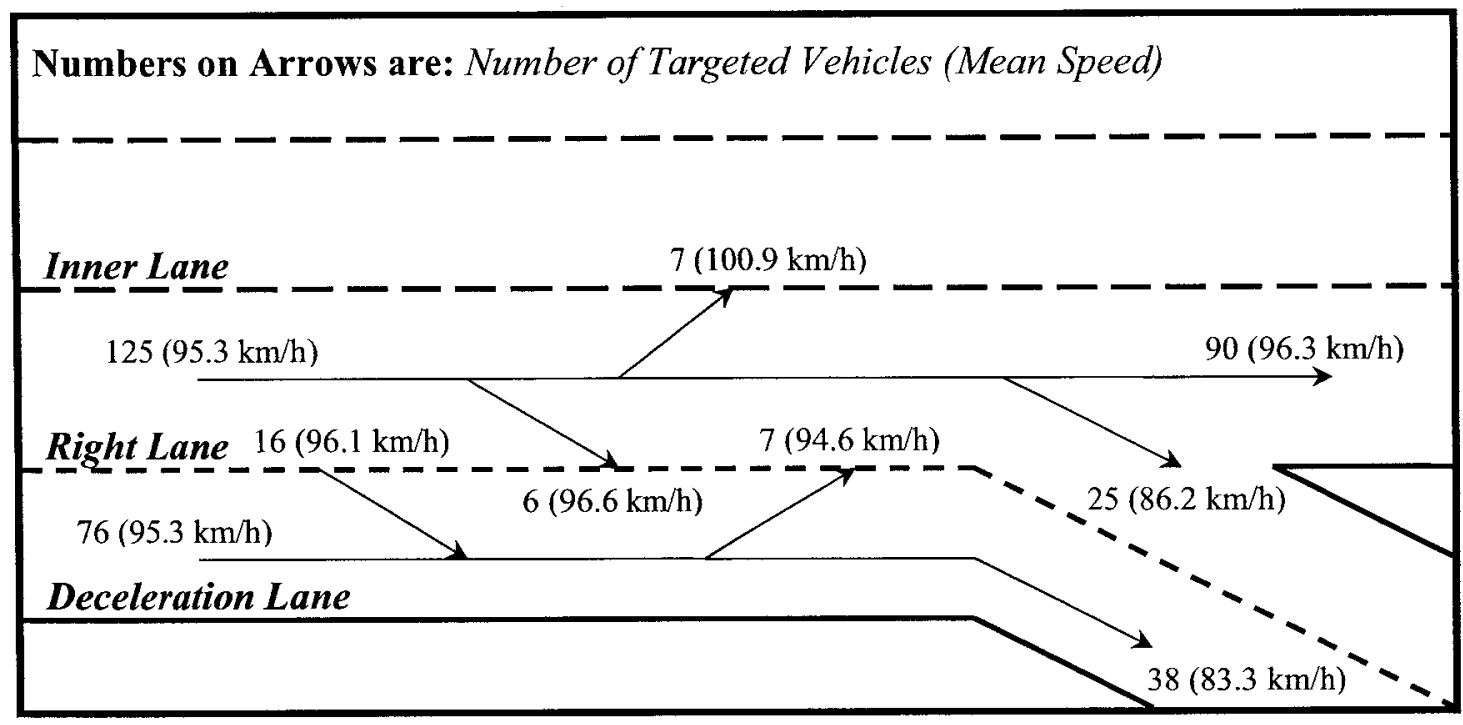

Figure A-9: Vanier Pkwy (W-NS) - Extended

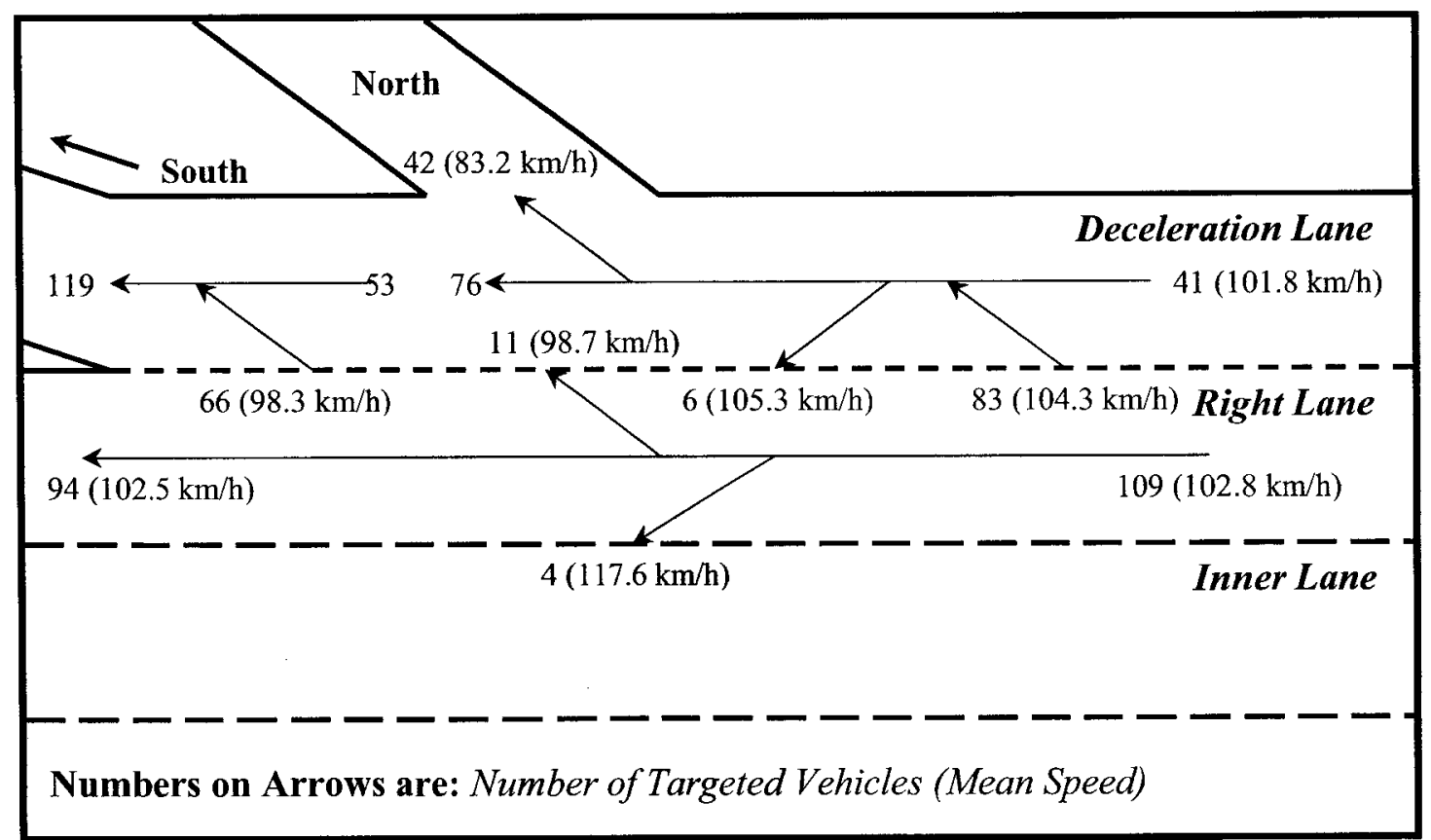

Figure A-10: Woodroffe Ave (E-NS) - Extended 


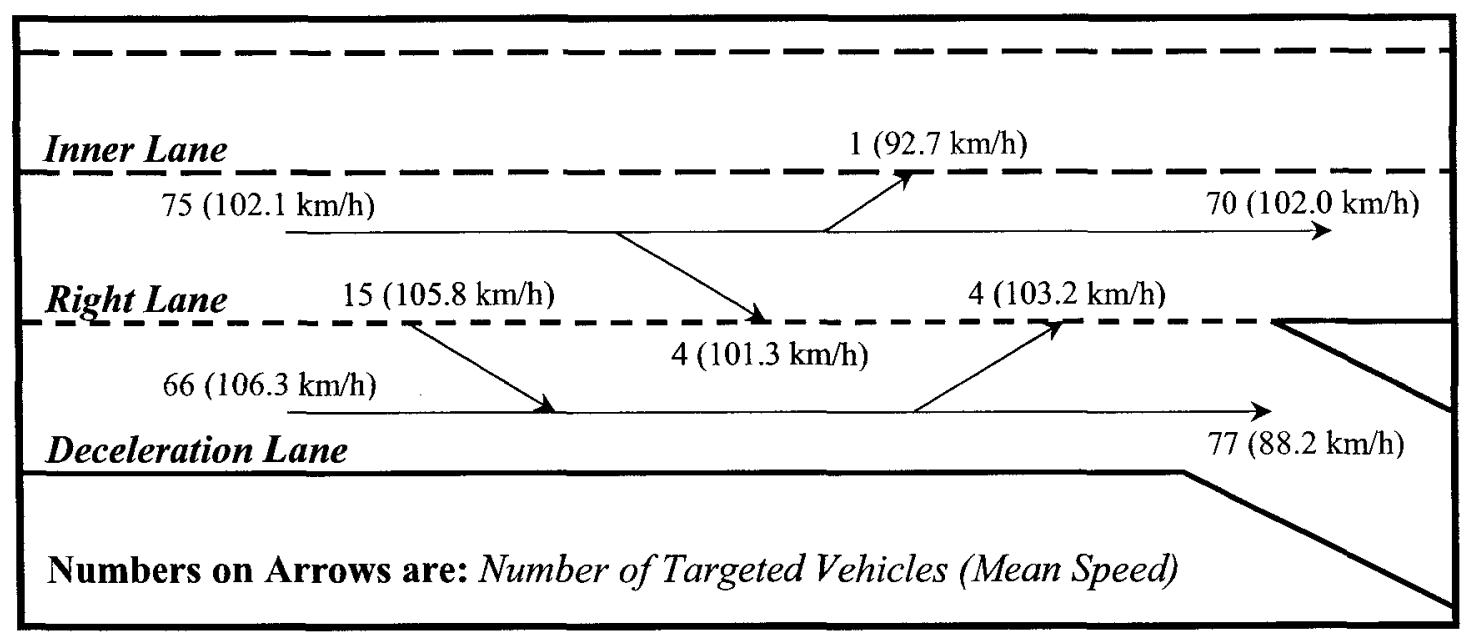

Figure A-11: Woodroffe Ave (W-NS) - Extended

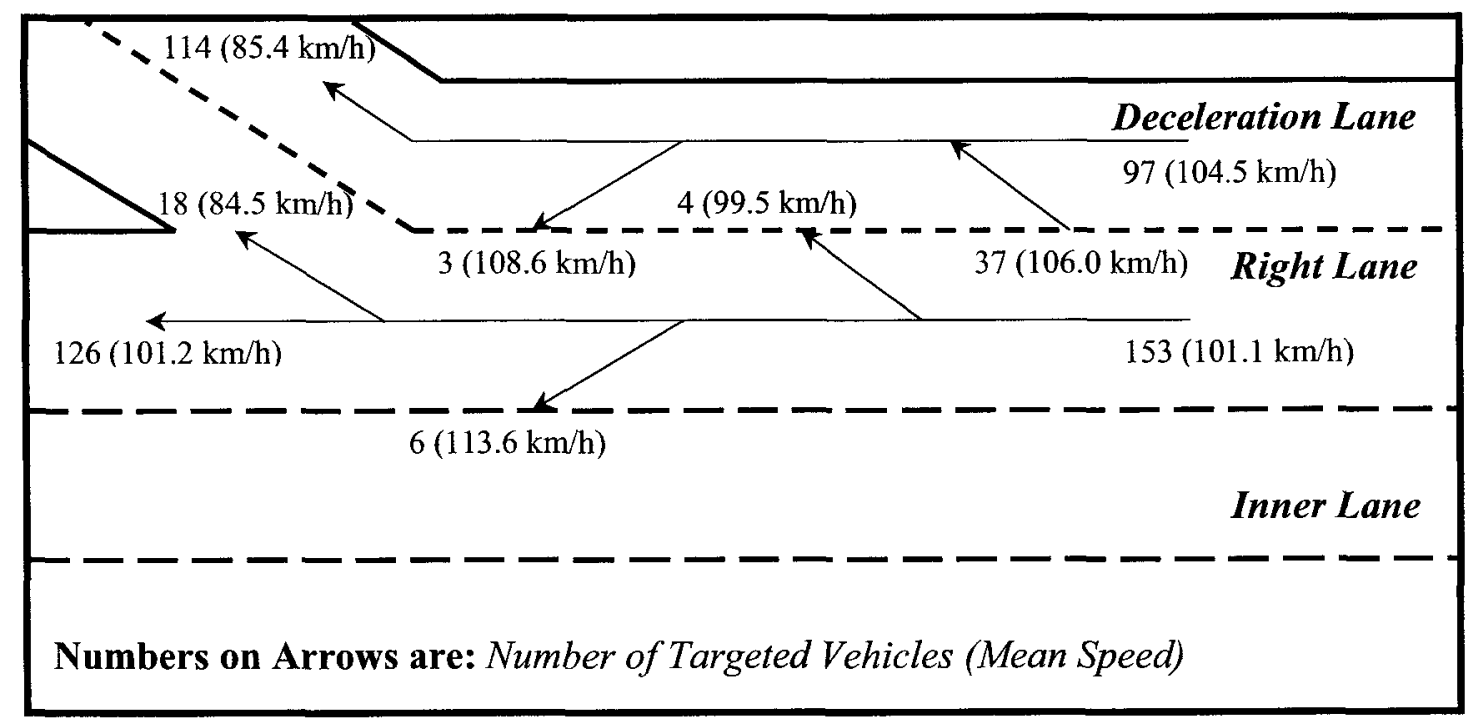

Figure A-12: Greenbank Rd /Pinecrest Rd (E-NS) - Extended 


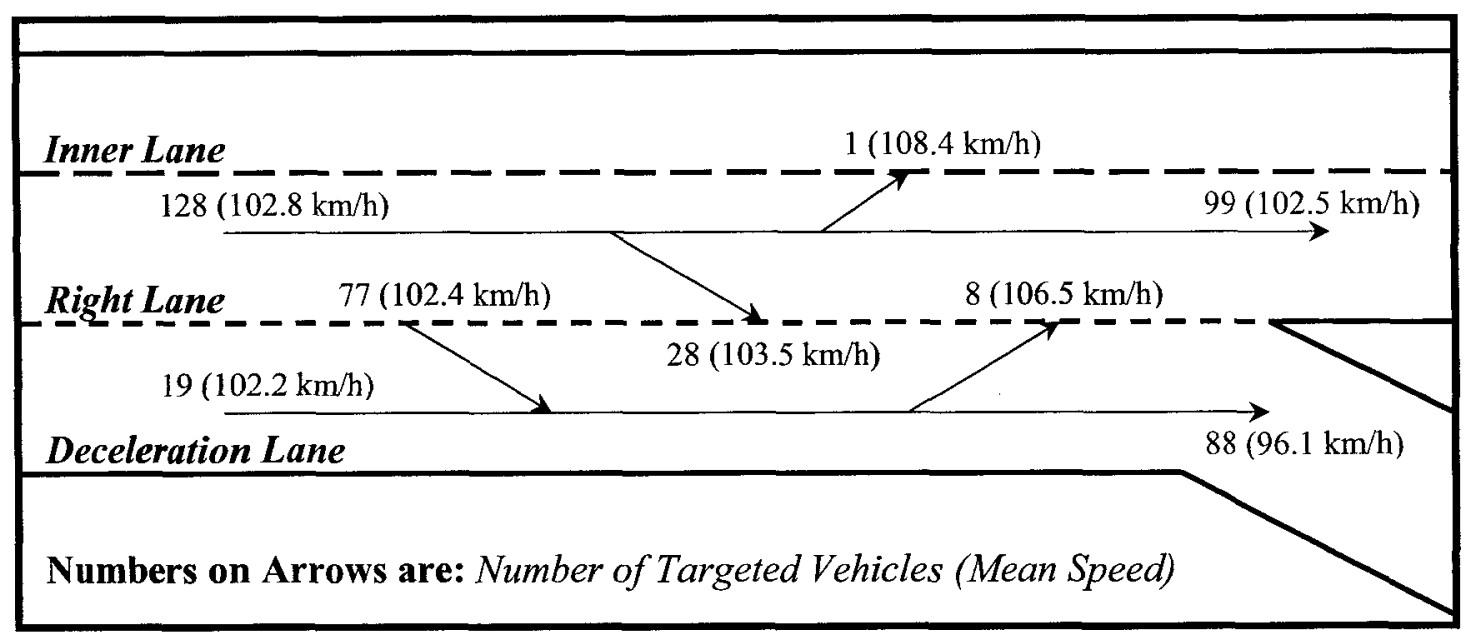

Figure A-13: Eagleson Rd (W-NS) - Extended 


\section{APPENDIX B: VEHICLE SPEED PROFILES ON DECELERATION SPEED CHANGE LANES}




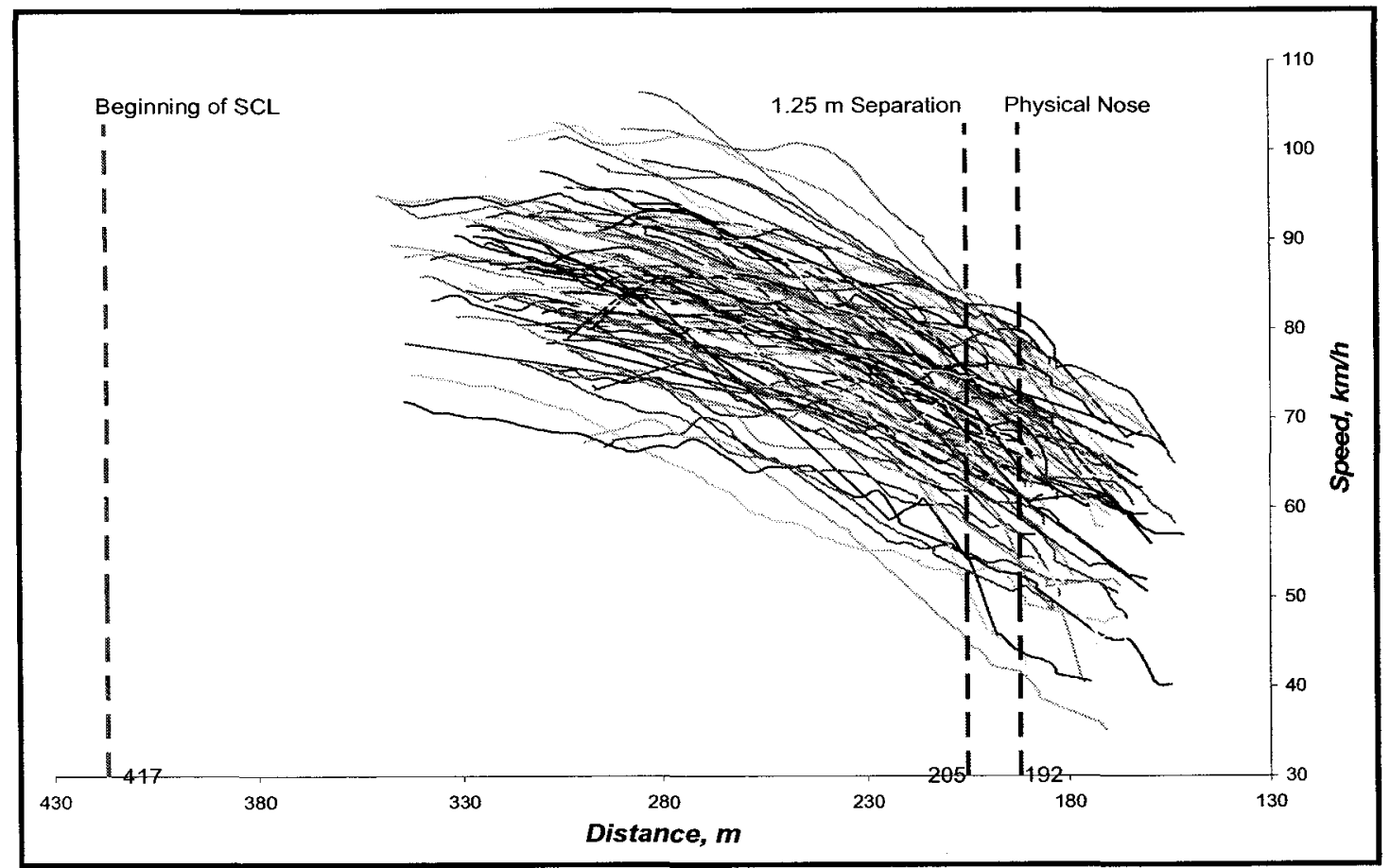

Figure B-1: Bronson Ave (W-NS) - Taper

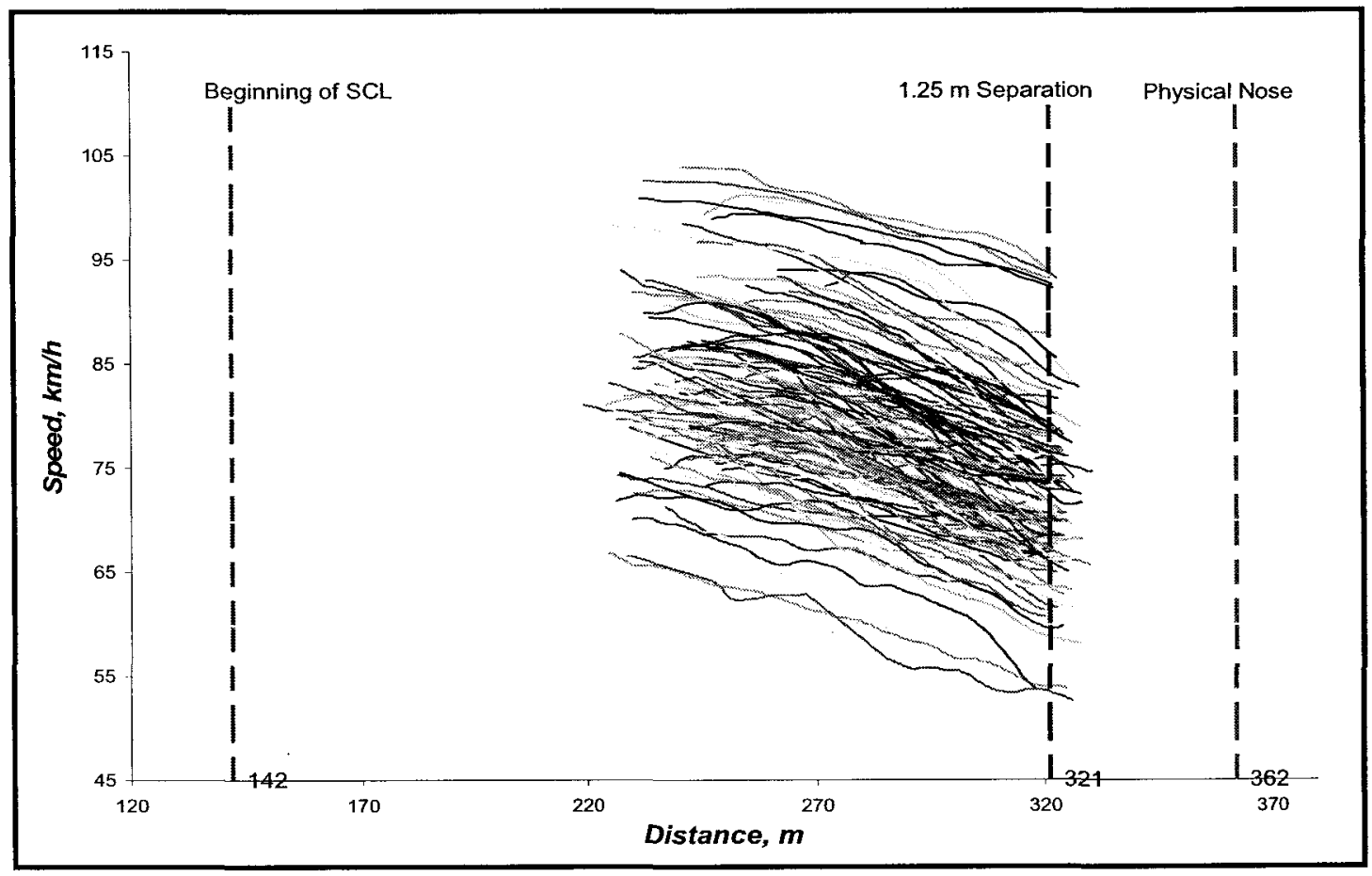

Figure B-2: Parkdale Ave (W-NS) - Taper 


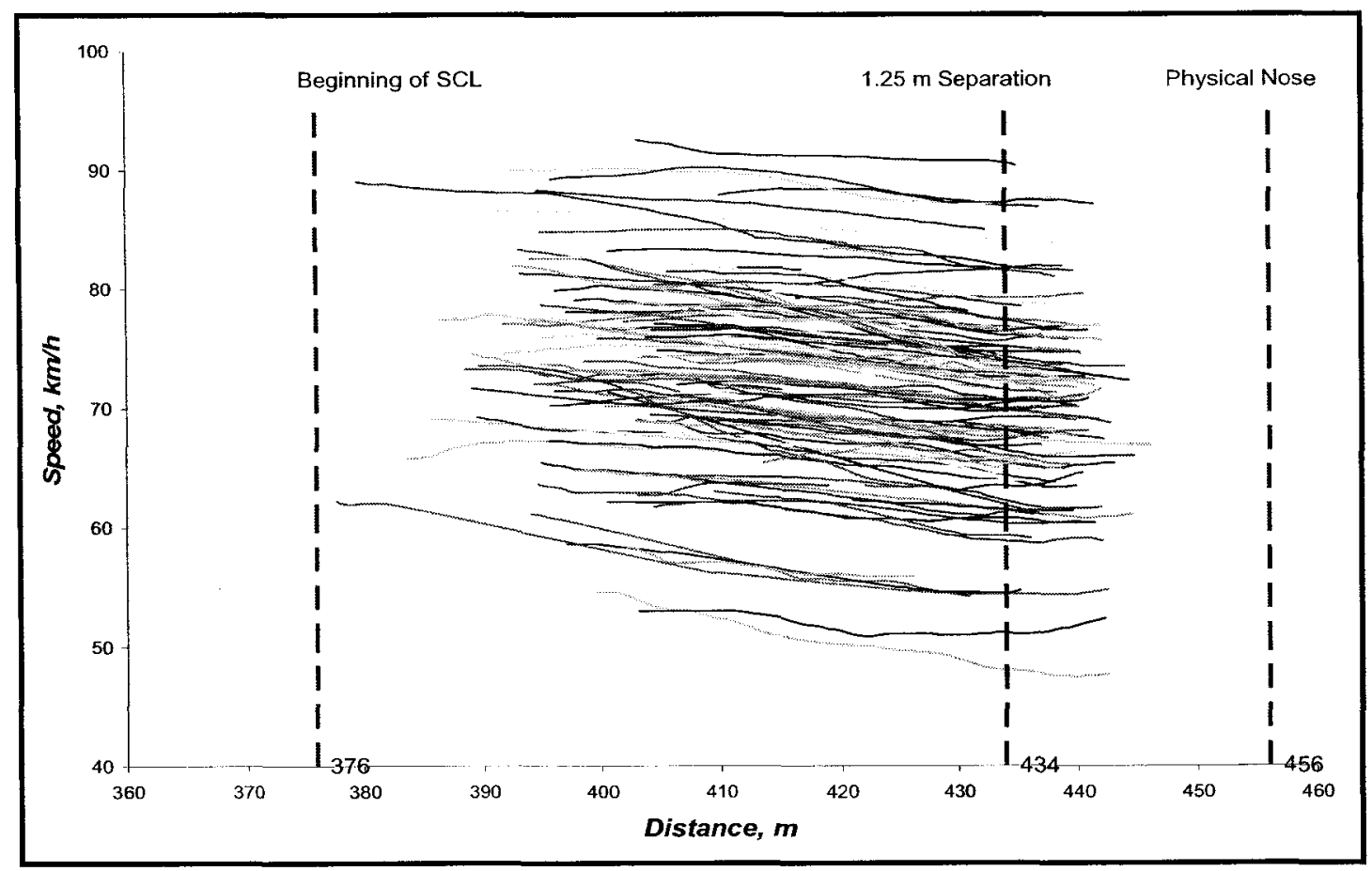

Figure B-3: Prom Island Park Dr (E-N) - Taper

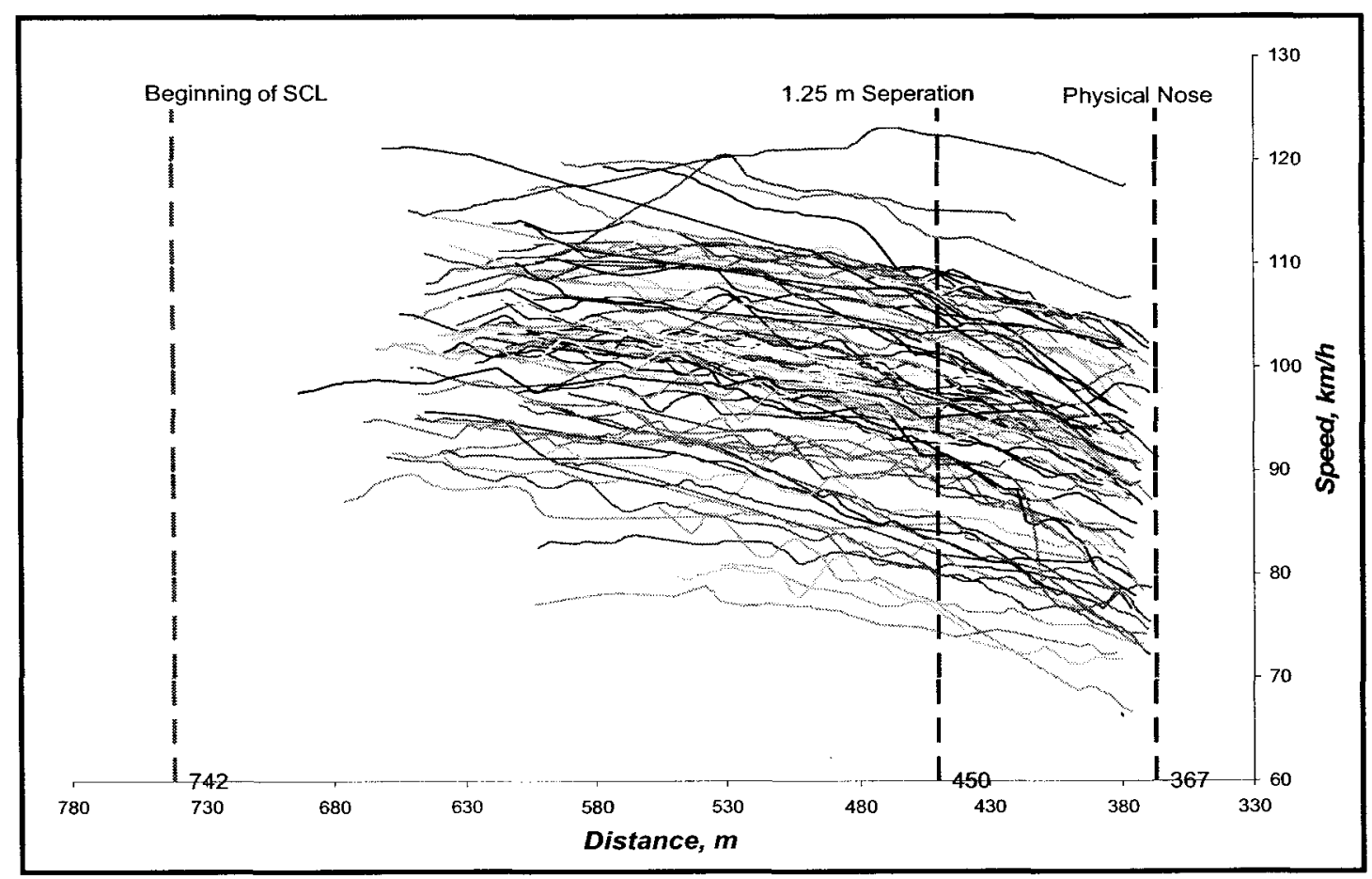

Figure B-4: Moodie Dr (W-NS) - Parallel 


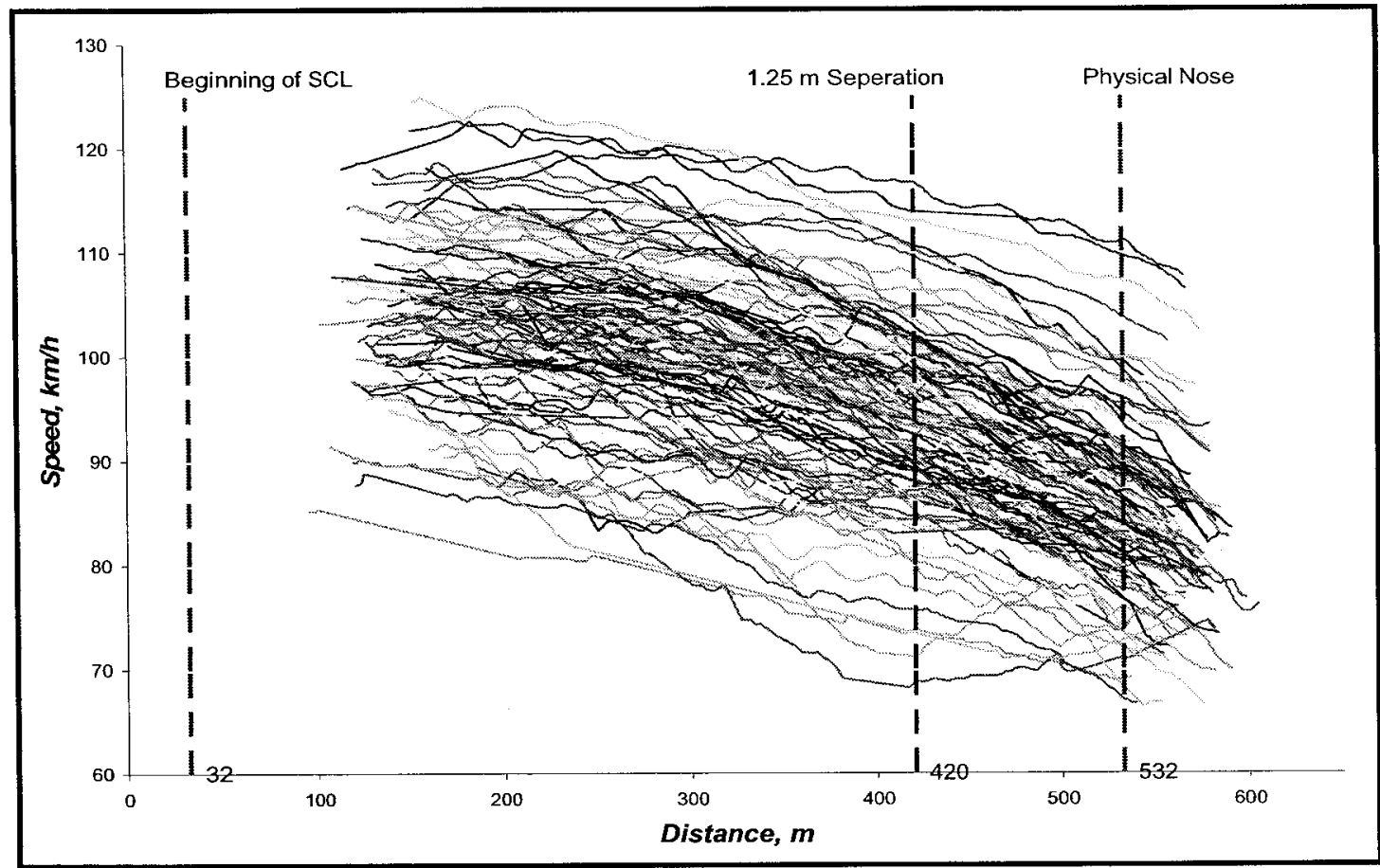

Figure B-5: Terry Fox Dr (E-NS) - Parallel

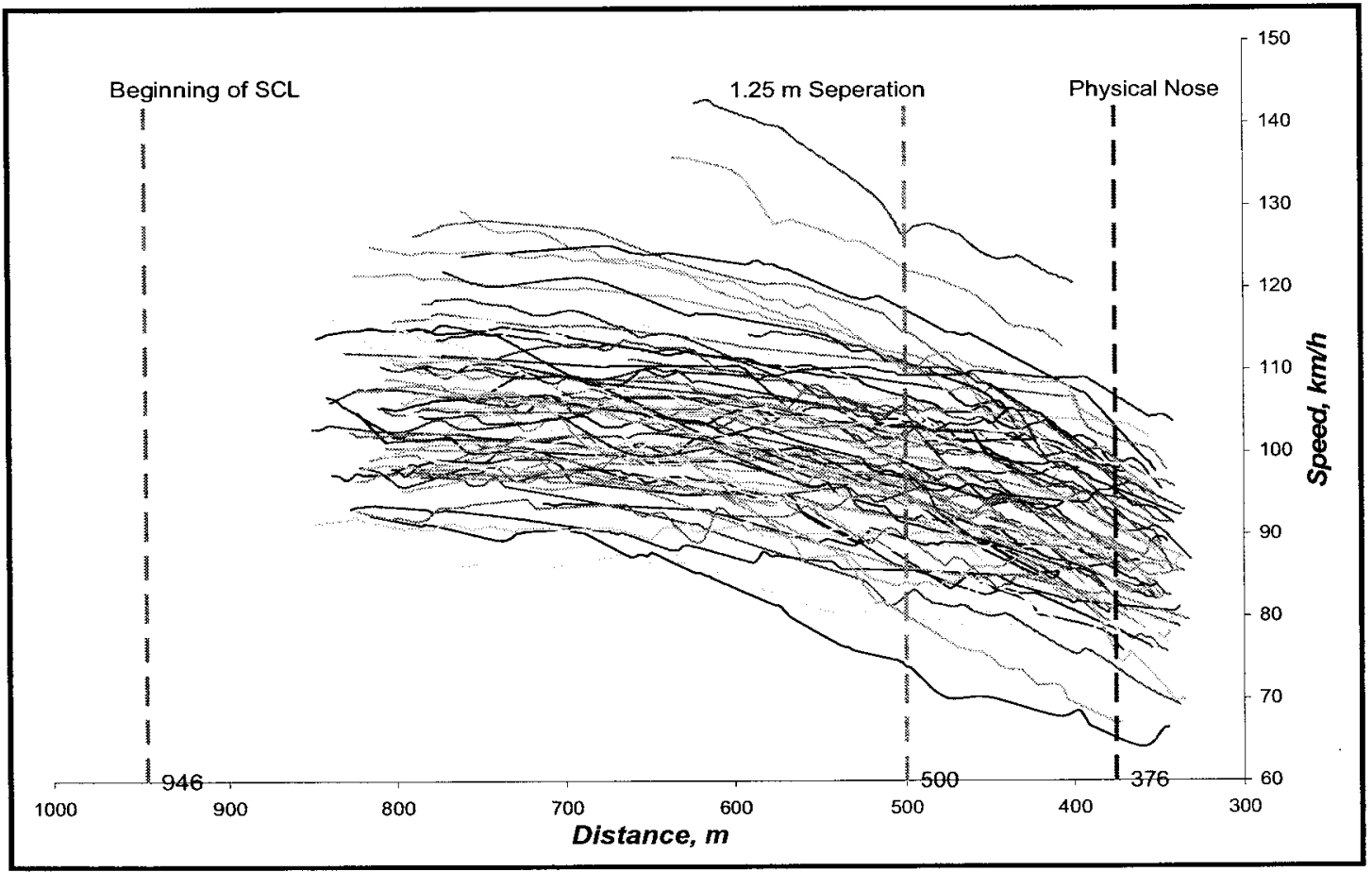

Figure B-6: Terry Fox Dr (W-NS) - Parallel 


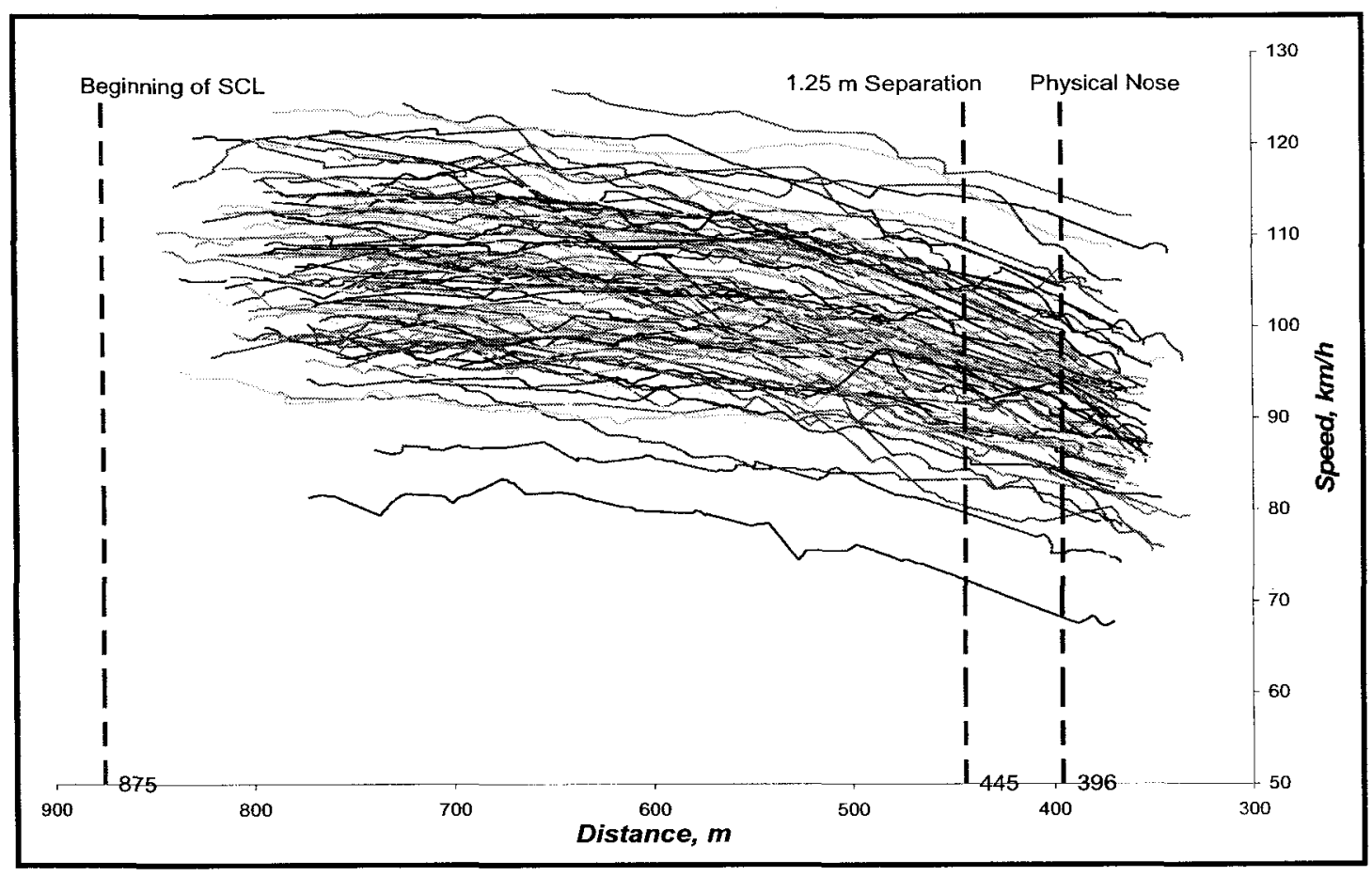

Figure B-7: Ch. Carp Rd (E-NS) - Parallel

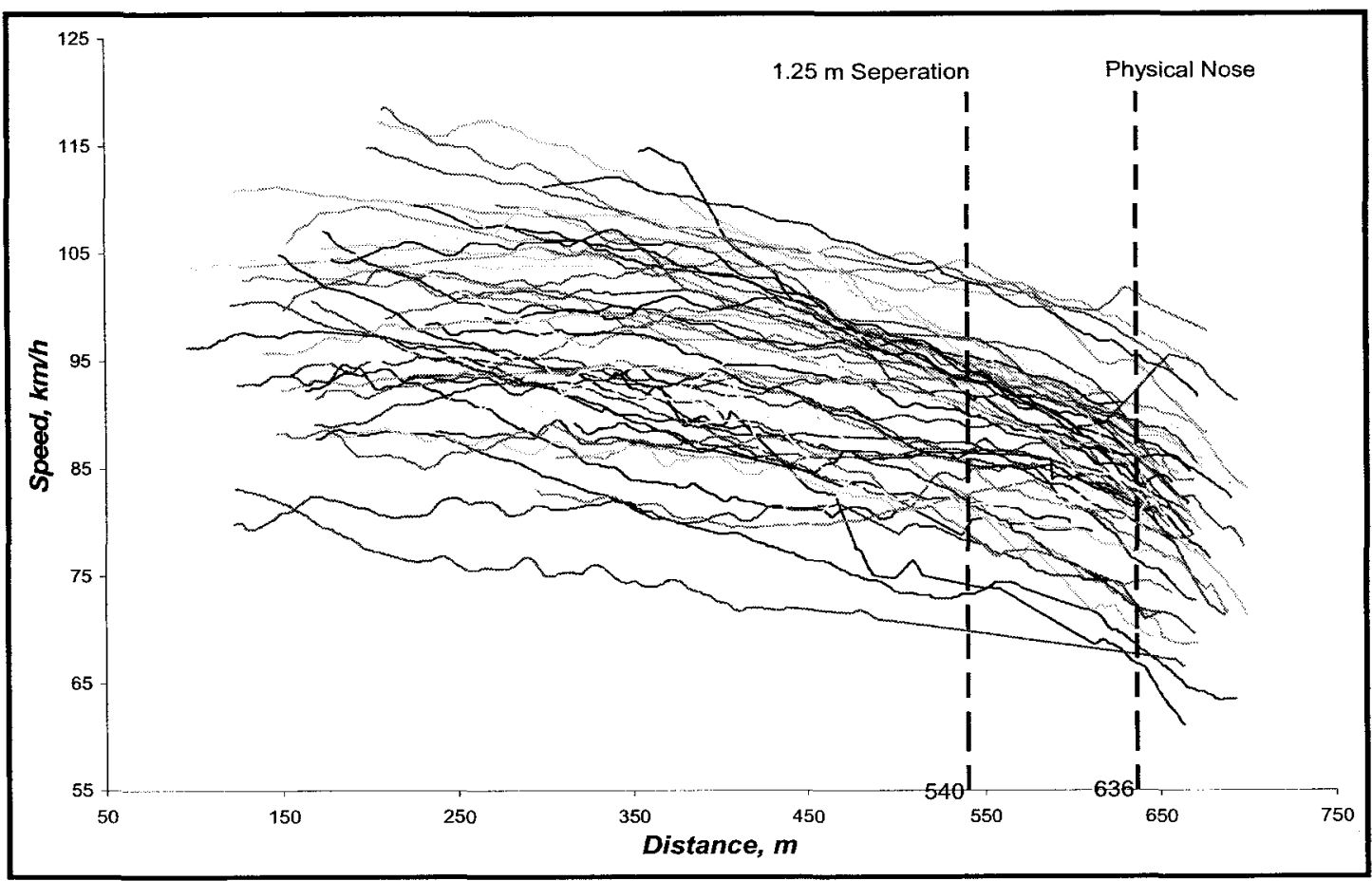

Figure B-8: St. Laurent Blvd (W-NS) - Extended 


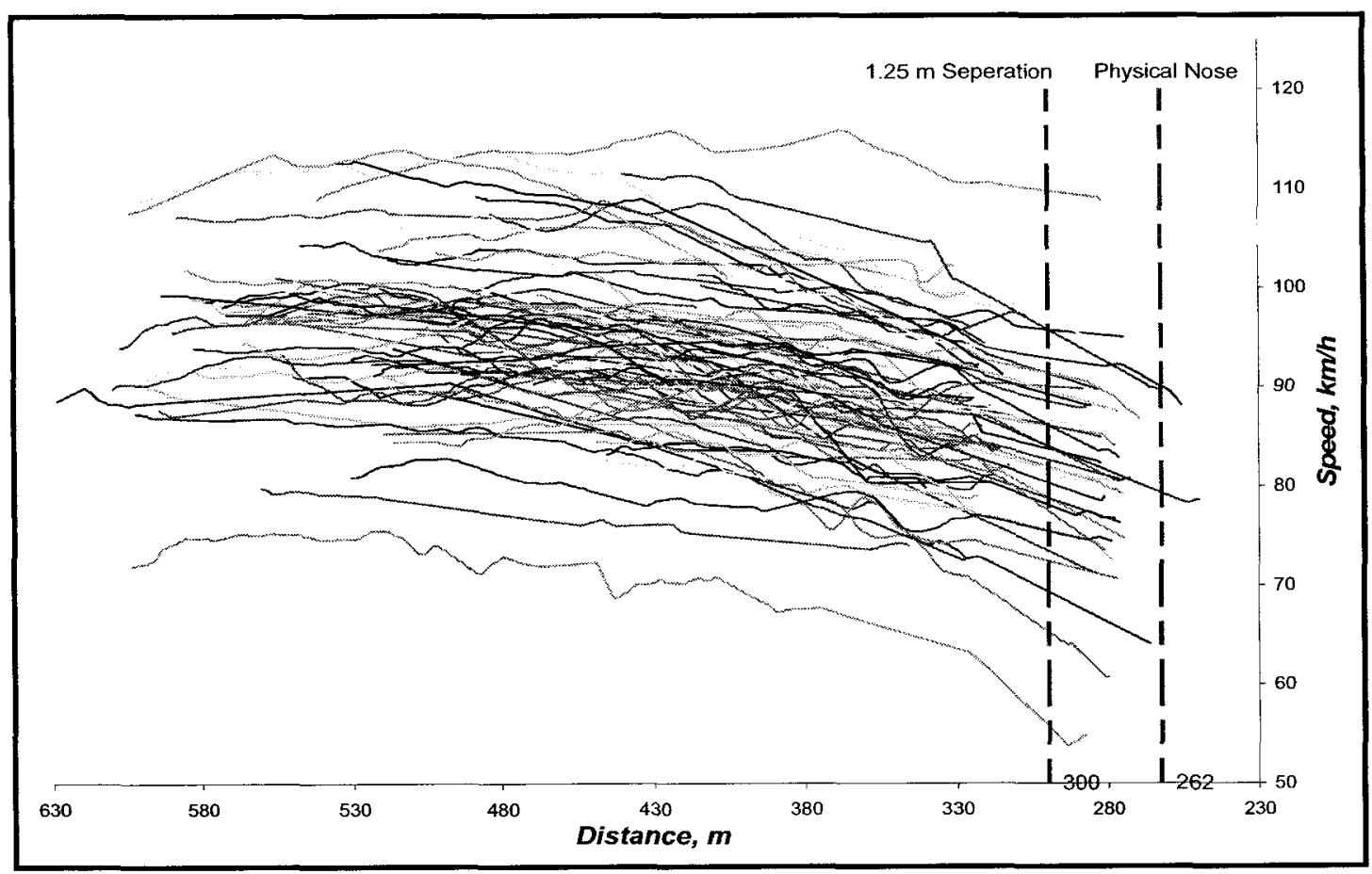

Figure B-9: Vanier Pkwy (W-NS) - Extended

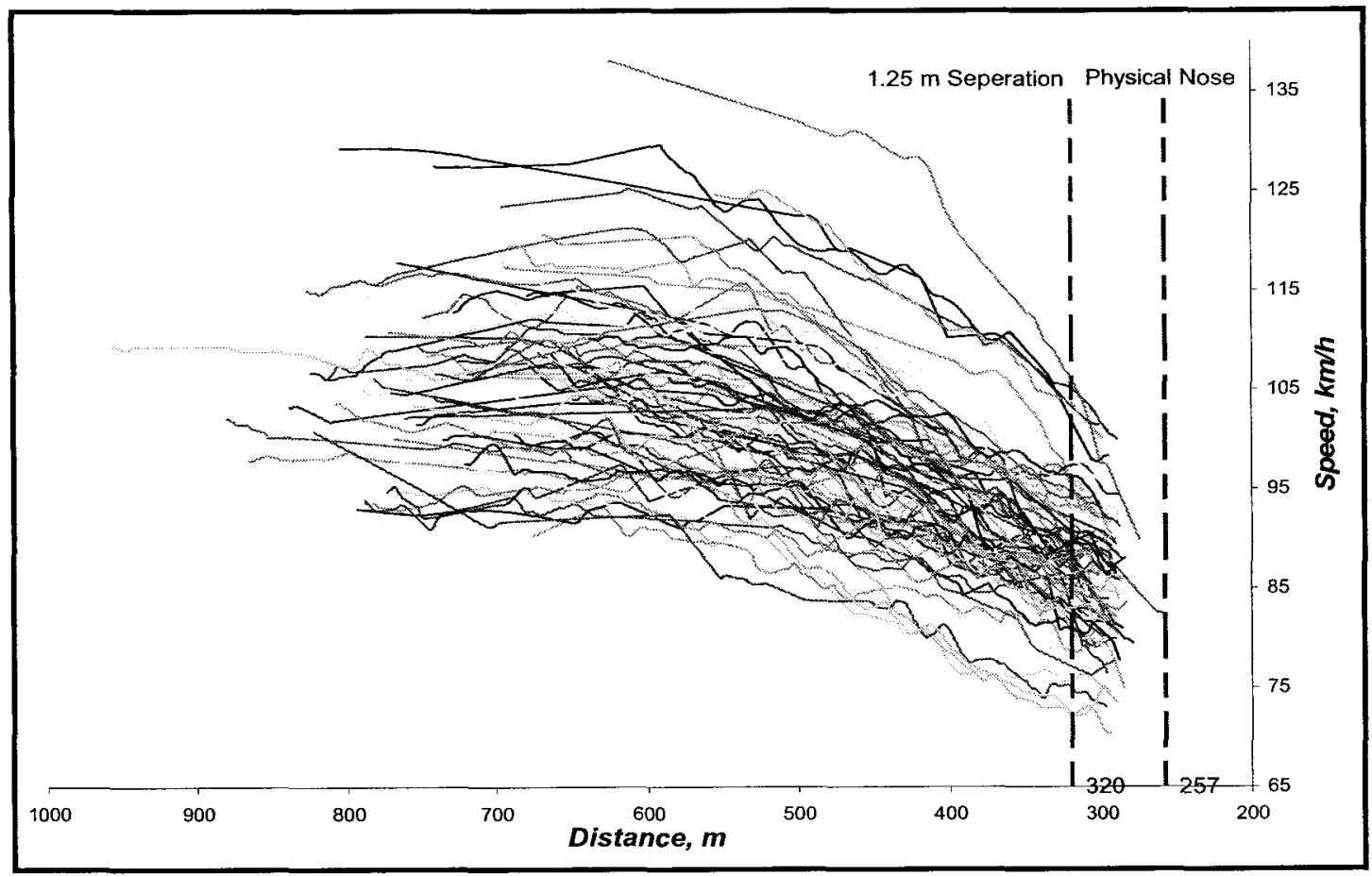

Figure B-11: Woodroffe Ave (W-NS) - Extended 


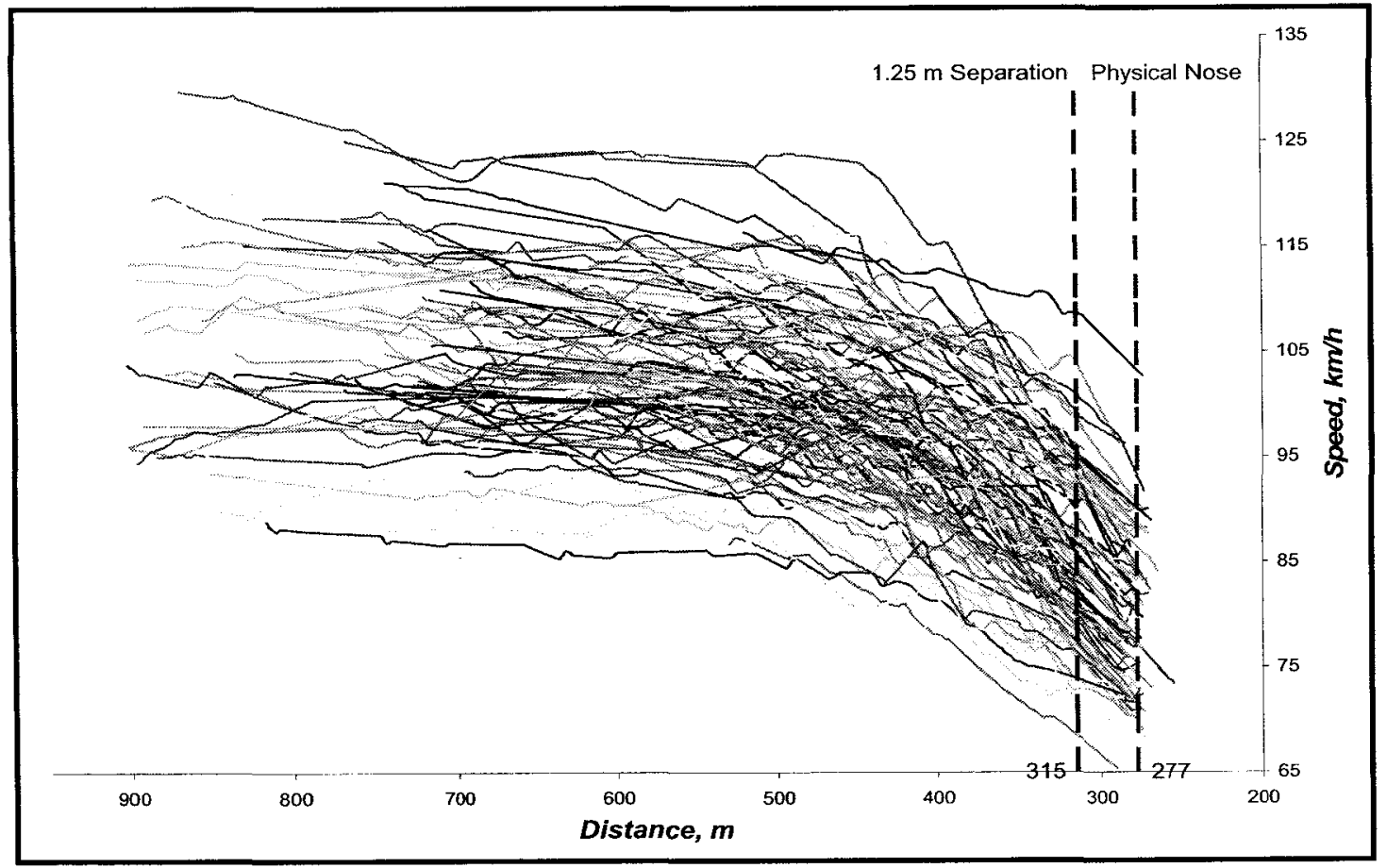

Figure B-12: Greenbank Rd /Pinecrest Rd (E-NS) - Extended

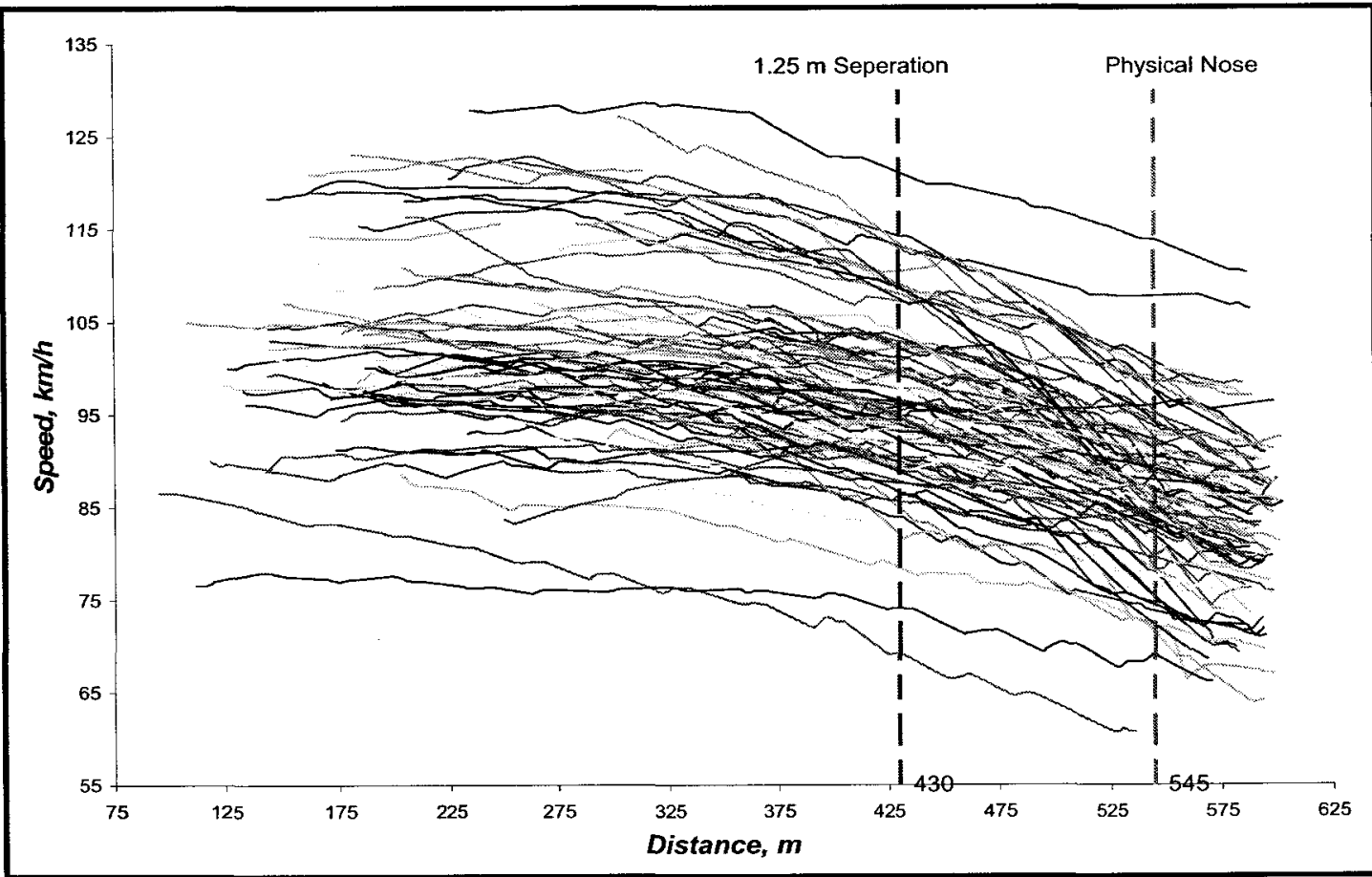

Figure B-13: Eagleson Rd (W-NS) - Extended 
APPENDIX C: DESCRIPTIVE SUMMARY OF SPEEDS, DECELERATION RATES, AND EFFECTIVE DECELERATION DISTANCES ON SELECTED STUDY SITES 
Table C-1: DESCRIPTIVE SUMMARY FOF SPEEDS ON LIMITED SCLS

\begin{tabular}{|c|c|c|c|c|c|c|c|c|c|c|}
\hline \multirow{3}{*}{ SCL Name } & \multirow{3}{*}{\multicolumn{2}{|c|}{ Statistics }} & \multicolumn{4}{|c|}{ Combined PC \& HV } & \multicolumn{4}{|c|}{ PC Only } \\
\hline & & & \multicolumn{8}{|c|}{ Speed $(\mathrm{km} / \mathrm{h})$} \\
\hline & & & Diverge & Maximum & Mean & Gore & Diverge & Maximum & Mean & Gore \\
\hline \multirow{8}{*}{$\begin{array}{l}\text { Bronson Ave } \\
\text { (W-NS) }\end{array}$} & \multirow{6}{*}{ Sample } & $\mathbf{N}$ & 138 & 90 & 90 & 88 & 124 & 85 & 85 & 84 \\
\hline & & 85th & 94.3 & 96.8 & 90.5 & 78.6 & 94.7 & 97.3 & 90.7 & 78.7 \\
\hline & & Min. & 65.1 & 69.9 & 62.0 & 52.6 & 65.1 & 69.9 & 62.0 & 52.6 \\
\hline & & Max. & 110.0 & 109.8 & 100.6 & 87.8 & 110.0 & 109.8 & 100.6 & 87.8 \\
\hline & & Mean & 86.9 & 88.0 & 81.3 & 70.5 & 87.8 & 88.3 & 81.6 & 70.9 \\
\hline & & SD & 8.54 & 8.28 & 8.49 & 7.69 & 8.14 & 8.29 & 8.40 & 7.51 \\
\hline & \multirow{2}{*}{ Population } & 85th & 95.7 & 96.6 & 90.1 & 78.4 & 96.6 & 96.9 & 90.3 & 78.7 \\
\hline & & 95th & 100.9 & 101.7 & 95.3 & 83.1 & 101.8 & 101.9 & 95.5 & 83.2 \\
\hline \multirow{8}{*}{$\begin{array}{l}\text { Parkdale Ave } \\
\qquad(W-N S)\end{array}$} & \multirow{6}{*}{ Sample } & $\mathbf{N}$ & 173 & 90 & 90 & 90 & 173 & 90 & 90 & 90 \\
\hline & & 85th & 92.5 & 93.1 & 89.4 & 82.1 & 92.5 & 93.1 & 89.4 & 82.1 \\
\hline & & Min. & 66.5 & 66.5 & 57.7 & 52.4 & 66.5 & 66.5 & 57.7 & 52.4 \\
\hline & & Max. & 108.9 & 103.9 & 99.3 & 93.9 & 108.9 & 103.9 & 99.3 & 93.9 \\
\hline & & Mean & 84.0 & 83.8 & 79.7 & 72.6 & 84.0 & 83.8 & 79.7 & 72.6 \\
\hline & & SD & 8.25 & 8.69 & 9.10 & 9.28 & 8.25 & 8.69 & 9.10 & 9.28 \\
\hline & \multirow{2}{*}{ Population } & 85th & 92.6 & 92.8 & 89.2 & 82.3 & 91.8 & 92.8 & 89.2 & 82.3 \\
\hline & & 95th & 97.6 & 98.1 & 94.7 & 87.9 & 96.7 & 98.1 & 94.7 & 87.9 \\
\hline \multirow{8}{*}{$\begin{array}{l}\text { Prom Island Park Dr } \\
\text { (E-N) }\end{array}$} & \multirow{6}{*}{ Sample } & $\mathbf{N}$ & 187 & 93 & 93 & 93 & 187 & 93 & 93 & 93 \\
\hline & & 85th & 80.4 & 81.9 & 80.0 & 78.8 & 80.4 & 81.9 & 80.0 & 78.8 \\
\hline & & Min. & 53.0 & 53.0 & 50.9 & 48.1 & 53.0 & 53.0 & 50.9 & 48.1 \\
\hline & & Max. & 94.3 & 92.5 & 91.3 & 90.5 & 94.3 & 92.5 & 91.3 & 90.5 \\
\hline & & Mean & 73.8 & 74.4 & 72.4 & 71.0 & 73.8 & 74.4 & 72.4 & 71.0 \\
\hline & & SD & 7.61 & 7.86 & 8.37 & 8.36 & 7.61 & 7.86 & 8.37 & 8.36 \\
\hline & \multirow{2}{*}{ Population } & 85th & 81.6 & 82.5 & 81.1 & 79.7 & 82.7 & 82.5 & 81.1 & 79.7 \\
\hline & & 95th & 86.3 & 87.3 & 86.2 & 84.8 & 87.7 & 87.3 & 86.2 & 84.8 \\
\hline & & $\mathbf{N}$ & 98 & 92 & 92 & 92 & 90 & 85 & 85 & 85 \\
\hline & & 85th & 111.0 & 111.9 & 109.8 & 106.4 & 111.1 & 112.1 & 110.0 & 106.5 \\
\hline & & Min. & 77.1 & 78.5 & 77.1 & 74.4 & 77.1 & 78.5 & 77.1 & 74.4 \\
\hline Moodie Dr & Sample & Max. & 121.2 & 123.1 & 118.7 & 122.4 & 121.2 & 123.1 & 118.7 & 122.4 \\
\hline (W-NS) & & Mean & 101.9 & 102.8 & 99.9 & 96.6 & 102.6 & 103.6 & 100.7 & 97.3 \\
\hline & & SD & 8.87 & 9.24 & 9.56 & 9.60 & 8.60 & 8.91 & 9.01 & 9.29 \\
\hline & & 85th & 111.1 & 112.3 & 109.8 & 106.5 & 112.2 & 112.8 & 110.1 & 107.0 \\
\hline & pulation & 95th & 116.5 & 118.0 & 115.6 & 112.4 & 117.6 & 118.2 & 115.6 & 112.6 \\
\hline & & $\mathbf{N}$ & 169 & 117 & 117 & 117 & 157 & 107 & 107 & 107 \\
\hline & & 85th & 114.2 & 111.8 & 107.9 & 102.4 & 114.2 & 114.1 & 108.2 & 102.9 \\
\hline & Samnle & Min. & 81.6 & 85.4 & 77.0 & 68.8 & 81.6 & 85.4 & 77.0 & 68.8 \\
\hline Terry Fox Dr & Sample & Max. & 124.6 & 124.9 & 119.7 & 116.7 & 124.6 & 124.9 & 119.7 & 116.7 \\
\hline (E-NS) & & Mean & 104.1 & 104.0 & 99.3 & 93.7 & 104.5 & 104.5 & 99.8 & 94.2 \\
\hline & & SD & 8.65 & 8.55 & 9.16 & 9.76 & 8.76 & 8.64 & 9.18 & 9.91 \\
\hline & Pomulation & 85th & 113.1 & 112.9 & 108.8 & 103.8 & 112.6 & 113.5 & 109.3 & 104.5 \\
\hline & ropulation & 95th & 118.3 & 118.1 & 114.4 & 109.8 & 117.6 & 118.7 & 114.9 & 110.5 \\
\hline & & $\mathbf{N}$ & 79 & 77 & 77 & 78 & 76 & 73 & 73 & 74 \\
\hline & & 85th & 114.0 & 115.9 & 112.5 & 109.0 & 114.7 & 116.3 & 112.9 & 109.4 \\
\hline & Sample & Min. & 84.7 & 87.6 & 84.4 & 73.9 & 84.7 & 87.6 & 84.4 & 73.9 \\
\hline Terry Fox Dr & sampie & Max. & 129.3 & 135.8 & 129.2 & 126.6 & 135.8 & 135.8 & 129.2 & 126.6 \\
\hline (W-NS) & & Mean & 105.8 & 107.1 & 103.2 & 99.1 & 106.5 & 107.4 & 103.5 & 99.4 \\
\hline & & SD & 9.09 & 9.55 & 9.18 & 9.88 & 9.73 & 9.67 & 9.26 & 10.00 \\
\hline & Ponulation & 85th & 115.2 & 117.0 & 112.7 & 109.3 & 117.0 & 117.4 & 113.0 & 109.7 \\
\hline & Population & 95th & 120.8 & 122.8 & 118.3 & 115.3 & 123.0 & 123.3 & 118.7 & 115.8 \\
\hline & & $\mathbf{N}$ & 121 & 99 & 99 & 99 & 101 & 85 & 85 & 85 \\
\hline & & 85th & 114.8 & 116.3 & 111.8 & 104.6 & 115.7 & 116.5 & 112.0 & 105.6 \\
\hline & & Min. & 81.4 & 83.4 & 79.6 & 72.3 & 86.5 & 87.4 & 84.8 & 79.6 \\
\hline Ch. Carp Rd & Sample & Max. & 125.9 & 125.9 & 122.4 & 117.5 & 125.9 & 125.9 & 122.4 & 117.5 \\
\hline (E-NS) & & Mean & 106.2 & 107.7 & 103.4 & 96.8 & 107.9 & 109.0 & 104.4 & 97.8 \\
\hline & & SD & 8.43 & 8.32 & 8.28 & 8.52 & 7.77 & 7.77 & 7.82 & 7.91 \\
\hline & & 85th & 114.9 & 116.4 & 112.0 & 105.6 & 116.3 & 117.0 & 112.5 & 106.0 \\
\hline & & 95th & 120.0 & 121.4 & 117.0 & 110.8 & 121.2 & 121.8 & 117.3 & 110.8 \\
\hline
\end{tabular}


Table C-2: DESCRIPTIVE SUMMARY FOR DECELERATION RATES AND EFFECTIVE DECELERATION DISTANCE ON LIMITED SCLS

\begin{tabular}{|c|c|c|c|c|c|c|c|c|c|c|}
\hline \multirow{3}{*}{ SCL Name } & \multirow{3}{*}{\multicolumn{2}{|c|}{ Statistics }} & \multicolumn{4}{|c|}{ Combined PC \& HV } & \multicolumn{4}{|c|}{ PC Only } \\
\hline & & & \multicolumn{3}{|c|}{ Deceleration Rate $\left(\mathrm{m} / \mathrm{s}^{2}\right)$} & \multirow{2}{*}{$\begin{array}{c}\text { Dec. } \\
\text { Dist. (m) }\end{array}$} & \multicolumn{3}{|c|}{ Deceleration Rate $\left(\mathrm{m} / \mathrm{s}^{2}\right)$} & \multirow{2}{*}{$\begin{array}{c}\text { Dec. } \\
\text { Dist. (m) }\end{array}$} \\
\hline & & & Overall & Maximum & Mean & & Overall & Maximum & Mean & \\
\hline \multirow{7}{*}{$\begin{array}{l}\text { Bronson Ave } \\
\text { (W-NS) }\end{array}$} & \multirow{5}{*}{ Sample } & $\mathbf{N}$ & 90 & 90 & 88 & 135 & 85 & 85 & 83 & 121 \\
\hline & & Min. & 0.050 & 1.025 & -0.466 & 50.6 & 0.050 & 1.025 & -0.466 & 50.6 \\
\hline & & Max. & 2.143 & 3.421 & 1.579 & 145.5 & 2.143 & 3.421 & 1.579 & 145.5 \\
\hline & & Mean & 0.998 & 2.223 & 0.671 & 99.9 & 0.991 & 2.219 & 0.661 & 100.3 \\
\hline & & SD & 0.41 & 0.50 & 0.38 & 20.79 & 0.41 & 0.51 & 0.39 & 20.73 \\
\hline & \multirow{2}{*}{ Population } & 85th & 1.417 & 2.738 & 1.067 & 121.4 & 1.415 & 2.747 & 1.064 & 123.0 \\
\hline & & 95th & 1.664 & 3.040 & 1.299 & 134.1 & 1.664 & 3.057 & 1.301 & 134.9 \\
\hline \multirow{8}{*}{$\begin{array}{c}\text { Parkdale Ave } \\
\text { (W-NS) }\end{array}$} & \multirow{6}{*}{ Sample } & $\mathbf{N}$ & 89 & 90 & 90 & 174 & 89 & 90 & 90 & 174 \\
\hline & & 85th & 1.226 & 2.326 & 1.055 & 108.4 & 1.226 & 2.326 & 1.055 & 108.4 \\
\hline & & Min. & 0.272 & 1.152 & -0.035 & 24.5 & 0.272 & 1.152 & -0.035 & 24.5 \\
\hline & & Max. & 1.685 & 3.275 & 1.651 & 138.4 & 1.685 & 3.275 & 1.651 & 138.4 \\
\hline & & Mean & 0.876 & 1.898 & 0.741 & 84.8 & 0.876 & 1.898 & 0.741 & 84.8 \\
\hline & & SD & 0.34 & 0.47 & 0.30 & 20.79 & 0.34 & 0.47 & 0.30 & 20.79 \\
\hline & \multirow{2}{*}{ Population } & 85th & 1.232 & 2.381 & 1.057 & 106.3 & 1.232 & 2.381 & 1.057 & 91.8 \\
\hline & & 95th & 1.441 & 2.665 & 1.242 & 119.0 & 1.441 & 2.665 & 1.242 & 100.3 \\
\hline & & $\mathbf{N}$ & 92 & 93 & 91 & 187 & 92 & 93 & 91 & 187 \\
\hline & & 85th & 0.747 & 1.715 & 0.660 & 39.1 & 0.747 & 1.715 & 0.660 & 39.1 \\
\hline & Sample & Min. & -0.509 & 0.004 & -0.525 & 8.3 & -0.509 & 0.004 & -0.525 & 8.3 \\
\hline Prom Island Park Dr & sample & Max. & 1.462 & 2.302 & 1.141 & 56.2 & 1.462 & 2.302 & 1.141 & 56.2 \\
\hline$(\mathrm{E}-\mathrm{N})$ & & Mean & 0.484 & 1.317 & 0.394 & 30.5 & 0.484 & 1.317 & 0.394 & 30.5 \\
\hline & & SD & 0.35 & 0.49 & 0.30 & 8.61 & 0.35 & 0.49 & 0.30 & 8.61 \\
\hline & Population & 85th & 0.843 & 1.824 & 0.709 & 39.4 & 0.843 & 1.824 & 0.709 & 41.8 \\
\hline & ropuanton & 95th & 1.053 & 2.122 & 0.895 & 44.6 & 1.053 & 2.122 & 0.895 & 48.1 \\
\hline & & $\mathbf{N}$ & 90 & 91 & 91 & 95 & 83 & 85 & 84 & 86 \\
\hline & & 85th & 0.465 & 1.876 & 0.410 & 199.7 & 0.460 & 1.871 & 0.411 & 198.8 \\
\hline & Sample & Min. & -0.330 & 0.408 & -0.309 & 66.4 & -0.330 & 0.265 & -0.309 & 82.5 \\
\hline Moodie Dr & & Max. & 0.711 & 2.799 & 0.698 & 243.6 & 0.711 & 2.799 & 0.698 & 225.6 \\
\hline (W-NS) & & Mean & 0.278 & 1.594 & 0.230 & 165.9 & 0.272 & 1.570 & 0.230 & 166.9 \\
\hline & & SD & 0.21 & 0.41 & 0.18 & 33.26 & 0.21 & 0.45 & 0.18 & 29.35 \\
\hline & Ponulation & 85th & 0.491 & 2.019 & 0.418 & 200.4 & 0.485 & 2.033 & 0.421 & 196.8 \\
\hline & Popration & 95th & 0.616 & 2.269 & 0.528 & 220.6 & 0.610 & 2.306 & 0.534 & 214.8 \\
\hline & & $\mathbf{N}$ & 116 & 115 & 117 & 169 & 106 & 105 & 107 & 157 \\
\hline & & 85th & 0.517 & 2.267 & 0.467 & 290.8 & 0.523 & 2.267 & 0.475 & 290.3 \\
\hline & Samole & Min. & -0.067 & 0.850 & -0.232 & 149.7 & -0.067 & 0.850 & -0.232 & 149.7 \\
\hline Terry Fox Dr & Dample & Max. & 0.720 & 3.133 & 0.827 & 324.7 & 0.720 & 3.133 & 0.827 & 324.7 \\
\hline (E-NS) & & Mean & 0.317 & 1.780 & 0.284 & 256.0 & 0.318 & 1.794 & 0.285 & 255.4 \\
\hline & & SD & 0.17 & 0.47 & 0.20 & 38.23 & 0.17 & 0.46 & 0.20 & 38.75 \\
\hline & Population & 85th & 0.491 & 2.266 & 0.489 & 295.6 & 0.496 & 2.272 & 0.495 & 296.7 \\
\hline & ropulamon & 95th & 0.594 & 2.552 & 0.609 & 318.9 & 0.601 & 2.553 & 0.619 & 321.3 \\
\hline & & $\mathbf{N}$ & 75 & 78 & 78 & 72 & 71 & 74 & 74 & 68 \\
\hline & & 85th & 0.393 & 2.236 & 0.387 & 329.8 & 0.397 & 2.241 & 0.395 & 329.7 \\
\hline & Sample & Min. & -0.099 & 0.556 & -0.275 & 207.7 & -0.099 & 0.556 & -0.275 & 207.7 \\
\hline Terry Fox Dr & Sampie & Max. & 0.646 & 2.900 & 0.795 & 349.4 & 0.646 & 2.900 & 0.795 & 346.8 \\
\hline$(\mathrm{W}-\mathrm{NS})$ & & Mean & 0.239 & 1.565 & 0.209 & 299.5 & 0.242 & 1.563 & 0.212 & 298.1 \\
\hline & & SD & 0.16 & 0.57 & 0.20 & 31.96 & 0.16 & 0.58 & 0.20 & 32.05 \\
\hline & Population & 85th & 0.403 & 2.156 & 0.415 & 332.7 & 0.409 & 2.167 & 0.423 & 339.5 \\
\hline & roputalton & 95th & 0.500 & 2.503 & 0.536 & 352.1 & 0.508 & 2.522 & 0.547 & 372.2 \\
\hline & & $\mathbf{N}$ & 98 & 99 & 97 & 116 & 84 & 85 & 84 & 96 \\
\hline & & 85th & 0.339 & 2.260 & 0.291 & 367.2 & 0.351 & 2.253 & 0.301 & 367.9 \\
\hline & Samole & Min. & -0.024 & 0.615 & -0.173 & 271.3 & -0.024 & 0.615 & -0.273 & 275.3 \\
\hline Ch. Carp Rd & sampre & Max. & 0.533 & 3.053 & 0.487 & 401.1 & 0.533 & 3.053 & 0.487 & 401.1 \\
\hline (E-NS) & & Mean & 0.226 & 1.687 & 0.154 & 334.5 & 0.240 & 1.701 & 0.157 & 336.3 \\
\hline & & SD & 0.12 & 0.51 & 0.13 & 29.24 & 0.11 & 0.50 & 0.15 & 28.99 \\
\hline & P & 85th & 0.346 & 2.216 & 0.293 & 364.8 & 0.356 & 2.221 & 0.309 & 368.1 \\
\hline & $10 n$ & 95th & 0.416 & 2,526 & 0.375 & 382.6 & 0.423 & 2.527 & 0.398 & 386.6 \\
\hline
\end{tabular}




\begin{tabular}{|c|c|c|c|c|c|c|c|c|c|c|c|c|c|c|c|c|c|c|c|}
\hline & Table C-3 (a): D & ESCRIPTIVE & SUMM & RY FOR & SPEEDS & N EXTEND & ED SCI & (COMB & NED PC & HV) & & & & & & & & & \\
\hline & \multirow{3}{*}{ SCL Name } & \multirow{3}{*}{\multicolumn{2}{|c|}{ Statistics }} & \multicolumn{16}{|c|}{ Speed $(\mathrm{km} / \mathrm{h})$} \\
\hline & & & & \multicolumn{4}{|c|}{ Initial } & \multicolumn{4}{|c|}{ Maximum } & \multicolumn{4}{|c|}{ Mean } & \multicolumn{4}{|c|}{ Gore } \\
\hline & & & & \multicolumn{2}{|c|}{ Occupying SCL } & Diverging & All & \multicolumn{2}{|c|}{ Occupying SCL } & Diverging & \multirow{2}{*}{ All } & \multicolumn{2}{|c|}{ Occupying SCL } & \multirow{2}{*}{ Diverging } & \multirow{2}{*}{ All } & \multicolumn{2}{|c|}{ Occupying SCL } & Diverging & All \\
\hline & & & $\mathbf{N}$ & $\frac{\text { Depart }}{27}$ & $\frac{\text { Merged }}{1}$ & 43 & 70 & $\frac{\text { Depart }}{27}$ & $\frac{\text { Merged }}{1}$ & 31 & & $\frac{\text { Depart }}{27}$ & $\frac{\text { Merged }}{1}$ & & & $\frac{\text { Depart }}{27}$ & $\frac{\text { Merged }}{1}$ & 31 & 58 \\
\hline & & & 85th & 105.6 & & 107.6 & 106.7 & 107.0 & & 109.5 & 109.4 & 103.2 & & 104.1 & 103.8 & 97.8 & & 95.4 & 96.9 \\
\hline & & & Min. & 83.5 & 95.3 & 72.1 & 72.1 & 83.5 & 95.3 & 79.2 & 79.2 & 78.8 & 91.5 & 73.2 & 73.2 & 69.1 & 88.4 & 62.9 & 62.9 \\
\hline & St. Laurent Blvd & Sample & Max. & 117.2 & 95.3 & 118.5 & 118.5 & 117.3 & 95.3 & 118.6 & 118.6 & 109.2 & 91.5 & 108.9 & 109.2 & 104.2 & 88.4 & 102.8 & 104.2 \\
\hline & (W-NS) & & Mean & 99.3 & 95.3 & 96.6 & 97.7 & 100.9 & 95.3 & 98.9 & 99.9 & 96.1 & 91.5 & 94.0 & 95.2 & 89.8 & 88.4 & 87.7 & 88.6 \\
\hline & & & SD & 8.54 & & 9.83 & 9.36 & 8.46 & & 10.35 & 9.54 & 8.01 & & 9.40 & 8.68 & 9.04 & & 9.00 & 9.21 \\
\hline & & & 85th & 108.3 & & 106.8 & 107.4 & 109.8 & & 109.7 & 109.7 & 104.5 & & 103.8 & 104.2 & 99.4 & & 97.1 & 98.2 \\
\hline & & Population & 95th & 113.9 & & 112.7 & 113.1 & 115.3 & & 116.0 & 115.6 & 109.7 & & 109.5 & 109.5 & 105.3 & & 102.5 & 103.8 \\
\hline & & & $\mathbf{N}$ & 69 & 7 & 22 & 91 & 34 & 7 & 4 & 38 & 34 & 7 & 4 & 38 & 34 & 7 & 4 & 38 \\
\hline & & & 85th & 104.2 & & 101.4 & 103.7 & 101.7 & & & 101.6 & 98.6 & & & 98.2 & 92.3 & & & 91.8 \\
\hline & & Sample & Min. & 72.0 & 68.5 & 85.8 & 72.0 & 75.5 & 70.0 & 94.5 & 75.5 & 72.1 & 69.0 & 90.2 & 72.1 & 55.6 & 70.0 & 80.5 & 55.6 \\
\hline & Vanier Pkwy & Dampie & Max. & 111.5 & 122.7 & 112.6 & 112.6 & 115.8 & 122.7 & 101.4 & 115.8 & 113.3 & 120.1 & 96.3 & 113.3 & 109.8 & 119.3 & 89.9 & 109.8 \\
\hline & (W-NS) & & Mean & 95.7 & 95.4 & 96.3 & 95.5 & 95.7 & 99.3 & 98.3 & 96.0 & 91.9 & 96.2 & 93.3 & 92.0 & 82.8 & 94.6 & 87.3 & 83.3 \\
\hline & & & SD & 8.57 & 17.19 & 6.66 & 7.95 & 8.62 & 18.47 & 3.14 & 8.21 & 8.88 & 16.91 & 2.48 & 8.49 & 10.23 & 15.03 & 4.58 & 9.84 \\
\hline & & Ponulation & 85th & 104.5 & & 103.4 & 103.7 & 104.6 & & & 104.5 & 101.1 & & & 100.8 & 93.4 & & & 93.5 \\
\hline & & Population & 95th & 109.7 & & 107.7 & 108.6 & 109.9 & & & 109.5 & 106.5 & & & 106.0 & 99.6 & & & 99.5 \\
\hline & & & $\mathbf{N}$ & 62 & 4 & 19 & 80 & 62 & 4 & 15 & 76 & 62 & 4 & 15 & 76 & 62 & 4 & 15 & 77 \\
\hline & & & 85th & 114.6 & & 120.1 & 114.8 & 115.6 & & 119.4 & 116.4 & 106.7 & & 109.3 & 107.2 & 95.4 & & 97.9 & 95.7 \\
\hline & & Sammle & Min. & 90.3 & 100.0 & 78.0 & 78.0 & 93.1 & 100.4 & 91.2 & 91.2 & 78.4 & 94,7 & 80.7 & 78.4 & 72.2 & 86.4 & 72.1 & 72.1 \\
\hline & Woodroffe Ave & Sample & Max. & 129.3 & 111.9 & 138.0 & 129.3 & 129.6 & 111.9 & 138.0 & 129.6 & 118.1 & 111.5 & 128.6 & 118.1 & 105.2 & 111.5 & 105.8 & 105.8 \\
\hline & (W-NS) & & Mean & 106.3 & 107.0 & 104.9 & 105.5 & 107,6 & 107.8 & 106.6 & 106.9 & 98.6 & 105.6 & 98.7 & 98.2 & 88.1 & 103.2 & 88.6 & $\mathbf{8 8 . 2}$ \\
\hline & & & SD & 8.57 & 5.51 & 14.57 & 9.45 & 8.61 & 5.19 & 13.82 & 9.22 & 8.01 & 7.55 & 13.81 & 8.69 & 6.92 & 11.40 & 10.01 & 7.67 \\
\hline & & Ponulation & 85th & 115.1 & & 120.4 & 115.3 & 116.5 & & 121.5 & 116.5 & 106.9 & & 113.5 & 107.2 & 95.2 & & 99.4 & 96.2 \\
\hline & & Toputanon & 95th & 120.4 & & 130.1 & 121.1 & 121.7 & & 131.0 & 122.1 & 111.7 & & 123.0 & 112.5 & 99.4 & & 106.2 & 100.8 \\
\hline & & & $\mathbf{N}$ & 93 & 3 & 41 & 134 & 81 & 3 & 32 & 113 & 81 & 3 & 32 & 113 & 82 & 3 & 32 & 114 \\
\hline & & & 85th & 114.6 & & 115.8 & 115.0 & 115.9 & & 116.1 & 116.2 & 108.3 & & 105.2 & 108.3 & 93.8 & & 94.4 & 94.0 \\
\hline & & Sample & Min. & 84.8 & 93.3 & 88.7 & 84.8 & 84.8 & 98.2 & 88.7 & 84.8 & 79.4 & 95.9 & 77.5 & 77.5 & 60.4 & 97.6 & 72.1 & 60.4 \\
\hline & $\begin{array}{l}\text { Greenbank Rd } \\
\text { (E-NS) }\end{array}$ & & Max. & 123.6 & 130.0 & 129.8 & 129.8 & 124.2 & 130.0 & 129.8 & 129.8 & 116.7 & 129.5 & 120.4 & 120.4 & 106.3 & 128.7 & 108.6 & 108.6 \\
\hline & & & SD & $\frac{104.8}{8.92}$ & $\frac{108.4}{1920}$ & $\begin{array}{l}105.3 \\
9.37\end{array}$ & $\frac{104.9}{8.97}$ & $\frac{105.6}{934}$ & $\begin{array}{l}110.0 \\
1739 \\
1739\end{array}$ & $\begin{array}{l}106.4 \\
8.94\end{array}$ & $\begin{array}{l}106.1 \\
936\end{array}$ & $\begin{array}{l}98.0 \\
8.99\end{array}$ & $\begin{array}{r}107.9 \\
1076\end{array}$ & 97.8 & $\begin{array}{l}98.0 \\
91\end{array}$ & $\frac{85.2}{9.5}$ & 108.6 & 85.7 & 85.4 \\
\hline & & Ponulation & 85th & 114.0 & & 115.0 & 114.2 & 115.3 & & 115.7 & 115.8 & 107.3 & 10.10 & $\frac{0.90}{107.0}$ & $\frac{9.15}{107.5}$ & 94.6 & 11.40 & 94.6 & 9.91 \\
\hline & & Population & 95th & 119.5 & & 120.7 & 119.7 & 121.0 & & 121.1 & 121.5 & 112.7 & & 112.4 & 113,1 & 100.1 & & 99.8 & 100.1 \\
\hline & & & $\mathbf{N}$ & 11 & 8 & 105 & 116 & 11 & 8 & 77 & 88 & 11 & 8 & 77 & 88 & 11 & 8 & 77 & 88 \\
\hline & & & 85th & 104.6 & & 115.9 & 115.4 & 105.0 & & 116.3 & 116.0 & 100.8 & & 112.6 & 112.1 & 100.6 & & 107.9 & 107.6 \\
\hline & & & Min. & 88.9 & 73.5 & 76.6 & 76.6 & 900 & 79.8 & 78.0 & 78.0 & 86.9 & 77.2 & 76.4 & 76.4 & 82.5 & 79.7 & 69.2 & 69.2 \\
\hline & Eagleson Rd & Sample & Max. & 106.1 & 135.9 & 127.9 & 127.9 & 115.9 & 141.7 & 128.6 & 128.6 & 111.0 & 139.1 & 126.6 & 126.6 & 114.1 & 141.4 & 121.1 & 121.1 \\
\hline & (W-NS) & & Mean & 99.3 & 106.2 & 102.7 & 102.4 & 101.4 & 109.0 & 103.4 & 103.3 & 97.6 & 106.4 & 100.7 & 100.4 & 93.9 & 106.5 & 96.5 & 96.1 \\
\hline & & & SD & 5.51 & 19.29 & 10.06 & 9.75 & 7.07 & 19.64 & 10.59 & 10.32 & 6.47 & 20.01 & 10.33 & 10.05 & 9.28 & 21.54 & 10.18 & 10.10 \\
\hline & & & 85th & 105.3 & & 113.1 & 112.5 & 109.1 & & 114.4 & 114.0 & 104.7 & & 111.4 & 110.8 & 104.0 & & 107.1 & 106.6 \\
\hline & & Population & 95th & 109.3 & & 119.3 & 118.4 & 114.2 & & 120.8 & 120.3 & 109.3 & & 117.7 & 116.9 & 110.7 & & 113.3 & 112.7 \\
\hline
\end{tabular}




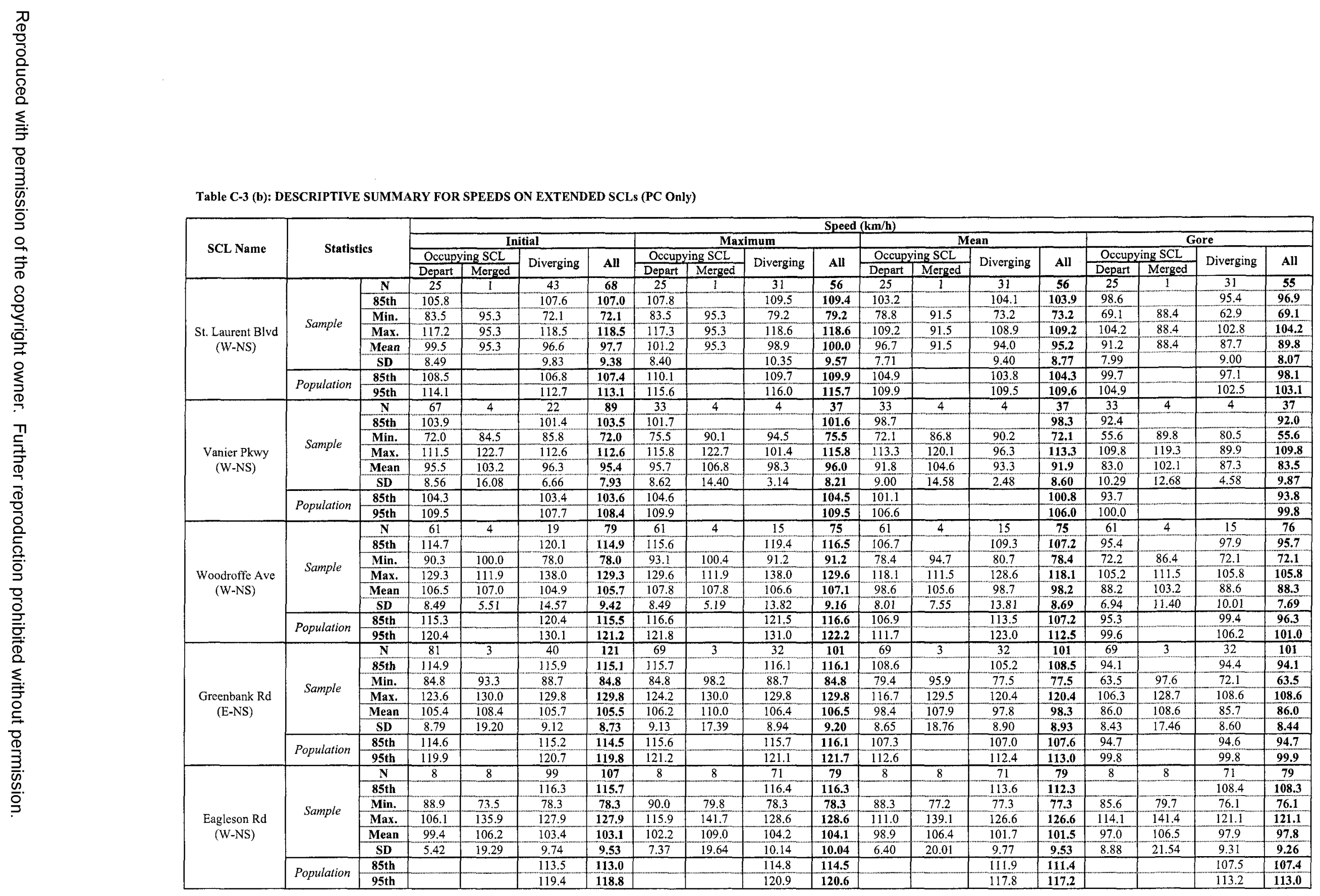


Table C-3 (c): DESCRIPTIVE SUMMARY FOR SPEEDS ON WOODROFFE (E-NS) SCL - (COMBINED PC \& HV)

\begin{tabular}{|c|c|c|c|c|c|c|c|c|c|c|c|c|c|c|c|c|c|c|}
\hline \multirow{3}{*}{$\begin{array}{c}\text { Section } \\
\text { (Depart) }\end{array}$} & \multirow{3}{*}{\multicolumn{2}{|c|}{ Statistics }} & \multicolumn{16}{|c|}{ Speed $(\mathrm{km} / \mathrm{h})$} \\
\hline & & & \multicolumn{4}{|c|}{ Initial } & \multicolumn{4}{|c|}{ Maximum } & \multicolumn{4}{|c|}{ Mean } & \multicolumn{4}{|c|}{ Exit/Final } \\
\hline & & & \multicolumn{2}{|c|}{\begin{tabular}{c|cc}
\multicolumn{2}{c|}{ Occupying SCL } \\
Depart & Merged
\end{tabular}} & \multirow{2}{*}{ Diverging } & All & \multicolumn{2}{|c|}{ Occupying SCL } & Diverging & All & \multicolumn{2}{|c|}{ Occupying SCL } & Diverging & All & \multicolumn{2}{|c|}{ Occupying SCL } & \multirow{2}{*}{ Diverging } & \multirow{2}{*}{ All } \\
\hline \multirow{8}{*}{$\begin{array}{l}\text { Before North-Exit } \\
\text { (North) }\end{array}$} & \multirow{6}{*}{ Sample } & $\mathbf{N}$ & $\frac{\text { Depart }}{14}$ & $\frac{\text { Merged }}{6}$ & & 41 & $\frac{\text { Depart }}{14}$ & $\frac{\text { Merged }}{6}$ & 27 & 41 & $\frac{\text { Depart }}{14}$ & $\frac{\text { Merged }}{6}$ & 28 & 42 & $\frac{\text { Depart }}{14}$ & $\frac{\text { Merged }}{6}$ & & \\
\hline & & $85 \mathrm{th}$ & 107.8 & & 106.6 & 107.4 & 110.1 & & 106.8 & 107.6 & 100.5 & & 100.5 & 100.5 & 88.2 & & 90.6 & 90.3 \\
\hline & & Min. & 85.1 & 97.4 & 85.2 & 85.1 & 85.1 & 98.0 & 85.2 & 85.1 & 79.1 & 96.4 & 78.7 & 78.7 & 72.2 & 95.9 & 73.6 & 72.2 \\
\hline & & Max. & 119.0 & 113.8 & 119.2 & 119.2 & 120.0 & 117.3 & 120.9 & 120.9 & 114.1 & 115.3 & 121.5 & 121.5 & 99.6 & 1116.4 & 110.3 & 100.8 \\
\hline & & Mean & 99.5 & 105.4 & 99.7 & 99.6 & 100.1 & 106.7 & 100.1 & 100.1 & 92.6 & 105.0 & 93.7 & 93.4 & 82.9 & 105.3 & 84.3 & 83.2 \\
\hline & & SD & 10.47 & 7.68 & 8.11 & 8.85 & 10.45 & 9.02 & 8.34 & 8.98 & 9.18 & 9.06 & 9.55 & 9.33 & 7.92 & 9.64 & 8.56 & 7.24 \\
\hline & \multirow{2}{*}{ Population } & 85th & 110.8 & & 108.2 & 108.8 & 111.4 & & 108.9 & 109.4 & 102.5 & & 103.8 & 103.0 & 91.4 & & 93.4 & 90.7 \\
\hline & & 95th & 118.0 & & 113.5 & 114.2 & 118,6 & & 114.3 & 114.8 & 108.9 & & 110.0 & 108.7 & 96.9 & & 98.9 & 95.1 \\
\hline & & $\mathrm{N}$ & 2 & & 55 & 75 & & 1 & 55 & 74 & 2 & & 55 & 74 & 2 & 11 & 55 & 76 \\
\hline & & 85 th & 11 & & 116.0 & 116.0 & & 4.7 & 116.3 & 115.6 & 10 & 3.7 & 112.0 & 111.3 & & 5.3 & 106.2 & 106.1 \\
\hline & & Min. & 73 & & 87.5 & 87.5 & & .0 & 87.5 & 87.5 & 69 & & 83.9 & 83.9 & & 3.6 & 79.5 & 70.6 \\
\hline Before North-Exit & Sample & Max. & 12 & & 130.4 & 130.4 & & 5.8 & 135.4 & 130.2 & 111 & 3.7 & 131.2 & 121.5 & & 3.5 & 123.1 & 123.1 \\
\hline (South) & & Mean & 10 & & 106.0 & 105.4 & & 3.3 & 106.8 & 105.9 & 99 & 6 & 102.3 & 101.6 & & 5.9 & 98.2 & 97.5 \\
\hline & & SD & 10 & & 9.50 & 9.32 & & 30 & 9.69 & 8.96 & 10 & 61 & 9.50 & 8.56 & & 72 & 8.68 & 8.97 \\
\hline & & 85th & $\overline{11}$ & 3.8 & 115.9 & 115.1 & & 5.3 & 116.8 & 115.2 & 11 & 0.9 & 112.1 & 110.4 & & 6.2 & 107.1 & 106.8 \\
\hline & Population & 95th & 12 & 1.0 & 121.6 & 120.7 & & 2.8 & 122.8 & 120.6 & 11 & 7.9 & 117.9 & 115.7 & & 2.7 & 112.4 & 112.3 \\
\hline & & $\mathrm{N}$ & 5 & 3 & 66 & 119 & & 3 & 66 & 118 & 5 & 2 & 66 & 118 & 5 & 3 & 66 & 119 \\
\hline & & 85 th & 10 & 3.0 & 108.5 & 108.4 & & 8.6 & 108.8 & 108.7 & 10 & 3.1 & 103.7 & 103.3 & & 3.5 & 99.6 & 98.8 \\
\hline & & Min. & 72 & .5 & 74.4 & 72.5 & & 5 & 81.3 & 72.5 & 70 & .4 & 75.9 & 70.4 & & 5.4 & 71.0 & 65.4 \\
\hline After North-Exit & Sample & Max. & 12 & 5.7 & 125.8 & 125.8 & & 9.6 & 125.8 & 125.8 & 11 & 0.3 & 121.1 & 121.1 & & 9.8 & 115.4 & 119.8 \\
\hline (South) & & Mean & 9 & .0 & 98.3 & 97.7 & & 2 & 99.6 & 98.7 & & 19 & 94.7 & 93.9 & & 8.0 & 89.4 & 88.8 \\
\hline & & SD & 11 & 71 & 9.55 & 10.54 & & 05 & 9.12 & 9.64 & 9. & 32 & 8.85 & 9.07 & & 83 & 9.86 & 10.28 \\
\hline & Ponulation & 85 th & 10 & 9.1 & 108.2 & 108.6 & & 9.6 & 109.1 & 108.7 & 10 & 2.5 & 103.9 & 103.3 & & 9.2 & 99.7 & 99.5 \\
\hline & Population & $95 \mathrm{th}$ & 11 & 5.2 & 114.0 & 115.1 & & 6.4 & 114.6 & 114.6 & 10 & 8.2 & 109.3 & 108.8 & & 5.8 & 105.7 & 105.7 \\
\hline & & $\mathbf{N}$ & & & 132 & 206 & & 4 & 120 & 194 & 7 & 4 & 120 & 194 & & $\overline{74}$ & 121 & 195 \\
\hline & & 85th & & 0.4 & 111.4 & 111.0 & & 0.4 & 111.8 & 111.7 & 10 & 4.1 & 107.3 & 106.3 & & 0.2 & 103.8 & 103.5 \\
\hline & & Min. & 72 & 5 & 74.4 & 72.5 & & 2.5 & 81.3 & 72.5 & 69 & .8 & 75.9 & 69.8 & & 5.4 & 71.0 & 65.4 \\
\hline & Sample & Max. & 12 & 5.7 & 130.4 & 130.4 & & 9.6 & 130.2 & 130.2 & 12 & 6.2 & 121.5 & 126.2 & & 9.8 & 123.1 & 123.1 \\
\hline (South) & & Mean & 98 & 5 & 101.6 & 100.5 & & 9.6 & 102.6 & 101.5 & 95 & 2 & 97.9 & 96.9 & & 0.2 & 93.4 & 92.2 \\
\hline & & SD & 11 & 66 & 10.57 & 11.05 & & .28 & 9.60 & 10.35 & 10 & 68 & 9.43 & 9.98 & & .06 & 10.27 & 10.66 \\
\hline & & 85th & 11 & 0.6 & 112.5 & 111.9 & & 1.3 & 112.6 & 112.2 & 10 & 6.3 & 107.7 & 107.2 & & 11.7 & 104.0 & 103.2 \\
\hline & Population & 95th & & 7.7 & 119.0 & 118.6 & & 8.2 & 118.4 & 118.5 & & 2.8 & 113.4 & 113.3 & & 8.4 & 110.3 & 109.7 \\
\hline
\end{tabular}


Table C-3 (d): DESCRIPTIVE SUMMARY FOR SPEEDS ON WOODROFFE (E-NS) SCL - (PC ONLY)

\begin{tabular}{|c|c|c|c|c|c|c|c|c|c|c|c|c|c|c|c|c|c|c|}
\hline \multirow{4}{*}{$\begin{array}{c}\text { Section } \\
\text { (Depart) }\end{array}$} & \multirow{4}{*}{\multicolumn{2}{|c|}{ Statistics }} & \multicolumn{16}{|c|}{ Speed $(\mathrm{km} / \mathrm{h})$} \\
\hline & & & \multicolumn{4}{|c|}{ Initial } & \multicolumn{4}{|c|}{ Maximum } & \multicolumn{4}{|c|}{ Mean } & \multicolumn{4}{|c|}{ Exit/Final } \\
\hline & & & \multirow{2}{*}{\multicolumn{2}{|c|}{ Occupying SCL }} & \multirow[b]{2}{*}{ Diverging } & \multirow[b]{2}{*}{ All } & \multicolumn{2}{|c|}{ Occupying SCL } & \multirow{2}{*}{ Diverging } & \multirow[b]{2}{*}{ All } & \multicolumn{2}{|c|}{ Occupying SCL } & \multirow{2}{*}{ Diverging } & \multirow{2}{*}{ All } & \multicolumn{2}{|c|}{ Occupying SCL } & \multirow{3}{*}{$\frac{\text { Diverging }}{28}$} & \multirow{2}{*}{ All } \\
\hline & & & Depart & & & & Depart & Merged & & & Depart & Merged & & & \begin{tabular}{l|l|} 
Depart \\
\end{tabular} & Merged & & \\
\hline \multirow{8}{*}{$\begin{array}{l}\text { Before North-Exit } \\
\text { (North) }\end{array}$} & \multirow{6}{*}{ Sample } & $\mathbf{N}$ & 12 & 5 & 27 & 39 & 12 & 5 & 27 & 39 & 12 & 5 & 28 & 40 & 12 & 5 & & 39 \\
\hline & & 85th & 109.0 & & 106.6 & 107.0 & 109.0 & & 106.8 & 107.3 & 97.8 & & 100.5 & 100.5 & 89.7 & & 90.6 & 90.4 \\
\hline & & Min. & 85.1 & 97.4 & 85.2 & 85.1 & 85.1 & 98.0 & 85.2 & 85.1 & 79.1 & 96.8 & 78.7 & 78.7 & 72.2 & 95.9 & 73.6 & 72.2 \\
\hline & & Max. & 119.0 & 113.8 & 119.2 & 119.2 & 120.0 & 117.3 & 120.9 & 120.9 & 114.1 & 115.3 & 121.5 & 121.5 & 99.6 & 116.4 & 110.3 & 100.8 \\
\hline & & Mean & 99.1 & 106.8 & 99.7 & 99.5 & 99.6 & 108.4 & 100.1 & 99.9 & 91.9 & 106.7 & 93.7 & 93.2 & 83.1 & 106.8 & 84.3 & 83.3 \\
\hline & & SD & 11.07 & 7.67 & 8.11 & 8.98 & 10.88 & 8.98 & 8.34 & 9.05 & 9.67 & 8.95 & 9.55 & 9.50 & 8.49 & 9.99 & 8.56 & 7.39 \\
\hline & \multirow{2}{*}{ Population } & 85th & 111.2 & & 108.2 & 108.8 & 111.4 & & 108.9 & 109.3 & 102.5 & & 103.8 & 103.0 & 92.4 & & 93.4 & 91.0 \\
\hline & & 95th & 119.0 & & 113.5 & 114.3 & 119.1 & & 114.3 & 114.8 & 109.3 & & 110.0 & 108.8 & 98.4 & & 98.9 & 95.5 \\
\hline & & $\mathrm{N}$ & & & 55 & 73 & & 8 & 55 & 72 & & & 55 & 72 & 1 & 8 & 55 & 73 \\
\hline & & 85th & & & 116.0 & 116.1 & & 6.1 & 116.3 & 115.8 & & & 112.0 & 111.5 & 10 & 5.0 & 106.2 & 106.3 \\
\hline & & Min. & & & 87.5 & 87.5 & & 5 & 87.5 & 87.5 & & & 83.9 & 83.9 & 87 & 3 & 79.5 & 79.5 \\
\hline Before North-Exit & Sample & Max. & & & 130.4 & 130.4 & & 5.8 & 135.4 & 130.2 & & & 131.2 & 121.5 & 113 & 3.5 & 123.1 & 123.1 \\
\hline (South) & & Mean & & & 106,0 & 105.5 & & 5.2 & 106.8 & 106.0 & & & 102.3 & 101.7 & 97 & 4 & 98.2 & 98.0 \\
\hline & & SD & & & 9.50 & 9.39 & & 45 & 9.69 & 9.02 & & & 9.50 & 8.58 & 7.6 & 69 & 8.68 & 8.40 \\
\hline & & 85th & & & 115.9 & 115.2 & & $\overline{5.3}$ & 116.8 & 115.3 & & & 112.1 & 110.6 & 10 & 5.6 & 107.1 & 106.7 \\
\hline & Population & $95 \mathrm{th}$ & & & 121,6 & 121.0 & & 1.6 & 122.8 & 120.8 & & 5.0 & 117.9 & 115.8 & 111 & 0.8 & 112.4 & 111.8 \\
\hline & & $\mathbf{N}$ & & & 66 & 117 & & 1 & 66 & 116 & & & 66 & 115 & 5 & 1 & 66 & 116 \\
\hline & & 85th & & & 108.5 & 108.4 & & 8.9 & 108.8 & 108.7 & & & 103.7 & 103.1 & 98 & 6.6 & 99.6 & 98.6 \\
\hline & & Min. & & & 74.4 & 74.3 & & .6 & 81.3 & 81.3 & & & 75.9 & 75.9 & 65 & 4 & 71.0 & 65.4 \\
\hline After North-Exit & Sample & Max. & & & 125.8 & 125.8 & & 9.6 & 125.8 & 125.8 & & & 121.1 & 112.2 & 115 & 9.8 & 115.4 & 115.4 \\
\hline (South) & & Mean & & & 98.3 & 98.1 & & 8.1 & 99.6 & 99.1 & & 7 & 94.7 & 94.1 & 88 & 8 & 89.4 & 88.9 \\
\hline & & SD & & & 9.55 & 10.13 & & .10 & 9.12 & 9.14 & & & 8.85 & 8.31 & 10. & 31 & 9.86 & 9.64 \\
\hline & & 85th & & & 108.2 & 108.6 & & 9.6 & 109.1 & 108.6 & & & 103.9 & 102.7 & 99 & 1.4 & 99.7 & 98.9 \\
\hline & Population & 95th & & & 114.0 & 114.8 & & 5.8 & 114.6 & 114.2 & & 7.6 & 109.3 & 107.7 & 10 & 5.7 & 105.7 & 104.7 \\
\hline & & $\mathbf{N}$ & & & 131 & 201 & & 9 & 120 & 189 & & & 120 & 189 & 6 & 9 & 121 & 190 \\
\hline & & 85 th & & & 111.5 & 111.1 & & 1.4 & 111.8 & 111.7 & & 4.0 & 107.3 & 106.6 & 10 & 0.1 & 103.8 & 103.5 \\
\hline & & Min. & & 3 & 74.4 & 74.3 & & .6 & 81.3 & 81.3 & & .1 & 75.9 & 75.9 & 65 & .4 & 71.0 & 65.4 \\
\hline & Sample & Max. & & 5.7 & 130.4 & 130.4 & & 9.6 & 130.2 & 130.2 & & 8.7 & 121.5 & 126.2 & 11 & 9.8 & 123.1 & 123.1 \\
\hline (South) & & Mean & & & 101.6 & 100.9 & & 0.7 & 102.6 & 101.9 & & & 97.9 & 97.3 & 91 & .0 & 93.4 & 92.5 \\
\hline & & SD & & 78 & 10.61 & 10.66 & & .22 & 9.60 & 9.85 & & & 9.43 & 9.53 & 10. & 37 & 10.27 & 10.35 \\
\hline & & 85 th & & 1.7 & 112.6 & 111.9 & & 1.3 & 112.6 & 112.1 & & & 107.7 & 107.1 & 10 & $\frac{1.8}{1.8}$ & 104.0 & 103.2 \\
\hline & Population & 95th & & 7.2 & 119.0 & 118.4 & & 7.5 & 118.4 & 118.1 & & 0.6 & 113.4 & 113.0 & 108 & 8.1 & 110.3 & 109.6 \\
\hline
\end{tabular}




\begin{tabular}{|c|c|c|c|c|c|c|c|c|c|c|c|c|c|c|c|c|c|}
\hline \multirow{4}{*}{ SCL Name } & \multirow{4}{*}{\multicolumn{2}{|c|}{ Statistics }} & \multicolumn{12}{|c|}{ Deceleration Rate $\left(\mathrm{m} / \mathrm{s}^{2}\right)$} & \multirow{2}{*}{\multicolumn{3}{|c|}{ Deceleration Distance (m) }} \\
\hline & & & \multicolumn{4}{|c|}{ Overall } & \multicolumn{4}{|c|}{ Maximum } & \multicolumn{4}{|c|}{ Mean } & & & \\
\hline & & & \multirow{2}{*}{\multicolumn{2}{|c|}{\begin{tabular}{c|c}
\multicolumn{2}{|c|}{ Occupying SCL } \\
Depart & Merged
\end{tabular}}} & \multirow{2}{*}{ Diverging } & \multirow{2}{*}{ All } & \multicolumn{2}{|c|}{ Occupying SCL } & \multirow{2}{*}{ Diverging } & \multirow{2}{*}{ All } & \multicolumn{2}{|c|}{ Occupying SCL } & \multirow{2}{*}{ Diverging } & \multirow{2}{*}{ All } & \multirow{2}{*}{$\mathrm{SCL}$} & \multirow{2}{*}{$\frac{\text { Diverging }}{42}$} & \multirow{2}{*}{$\begin{array}{l}\text { All } \\
69\end{array}$} \\
\hline & & & & & & & Depart & Merged & & & Depart & Merged & & & & & \\
\hline & & $\frac{N}{85 \text { th }}$ & $\frac{26}{0.262}$ & 1 & $\frac{31}{0.370}$ & $\frac{57}{0.362}$ & $\begin{array}{l}\frac{27}{2.157} \\
\end{array}$ & 1 & $\frac{31}{1.688}$ & $\frac{58}{1.702}$ & $\begin{array}{c}26 \\
0.269\end{array}$ & 1 & $\frac{31}{0.366}$ & 0.355 & $\frac{27}{412.4}$ & 394,3 & 409.4 \\
\hline & & Min. & -0.080 & 0.350 & 0.005 & -0.080 & 1.114 & 1.124 & 0.843 & 0.843 & -0.090 & 0.330 & -0.003 & -0.090 & 158.3 & 130.5 & 130.5 \\
\hline St. Laurent Blvd & Sample & Max. & 0.550 & 0.350 & 0.636 & 0.636 & 2.540 & 1.124 & 2.276 & 2.540 & 0.446 & 0.330 & 0.615 & 0.706 & 439.8 & 442.9 & 442.9 \\
\hline$(W-N S)$ & & Mean & 0.193 & 0.350 & 0.240 & 0.219 & 1.521 & 1.124 & 1.433 & 1.496 & 0.183 & 0.330 & 0.236 & 0.221 & 329.6 & 315.8 & 321.8 \\
\hline & & SD & 0.14 & & 0.16 & 0.15 & 0.46 & & 0.35 & 0.42 & 0.13 & & 0.17 & 0.16 & 84.71 & 82.53 & 82.61 \\
\hline & & 85th & 0.343 & & 0.408 & 0.379 & 2.012 & & 1.799 & 1.930 & 0.317 & & 0.407 & 0.391 & 419.265 & 401.3 & 407.4 \\
\hline & Population & 95th & 0.435 & & 0.507 & 0.473 & 2.314 & & 2.014 & 2.185 & 0.400 & & 0.508 & 0.491 & 474.156 & 451.6 & 457.7 \\
\hline & & $\mathbf{N}$ & 33 & 7 & 4 & 37 & 34 & 7 & 4 & 38 & 33 & 7 & 4 & 37 & 69 & 22 & 91 \\
\hline & & 85th & 0.556 & & 0.565 & 0.562 & 1.689 & & 1.779 & 1.710 & 0.421 & & 0.508 & 0.429 & 287.3 & 253.9 & 285.1 \\
\hline & & Min. & -0.033 & -0.457 & 0.059 & -0.033 & 1.102 & 0.565 & 1.119 & 1.102 & -0.063 & -0.407 & 0.022 & -0.063 & 92.8 & 26.8 & 26.8 \\
\hline Vanier Pkwy & Sample & Max. & 0.851 & 0.295 & 0.582 & $\mathbf{0 . 8 5 1}$ & 1.832 & 1.678 & 1.862 & 1.862 & 0.529 & 0.193 & 0.567 & 0.567 & 328.3 & 294.1 & 328.3 \\
\hline (W-NS) & & Mean & 0.351 & 0.012 & 0.375 & 0.354 & 1.514 & 1.256 & 1.582 & 1.535 & 0.230 & -0.038 & 0.326 & 0.240 & 227.2 & 167.6 & 212.8 \\
\hline & & SD & 0.20 & 0.29 & 0.24 & 0.20 & 0.23 & 0.39 & 0.32 & 0.23 & 0.17 & 0.22 & 0.23 & 0.18 & 57.43 & 81.92 & 68.67 \\
\hline & & 85th & 0.561 & & 0.677 & 0.565 & 1.752 & & 1.983 & 1.773 & 0.408 & & 0.619 & 0.425 & 286.7 & 254.7 & 283.9 \\
\hline & Population & 95th & 0.685 & & 0.943 & 0.689 & 1.893 & & 2.337 & 1,913 & 0.513 & & 0.877 & 0.534 & 321.6 & 308.6 & 325.7 \\
\hline & & $\mathbf{N}$ & 62 & 4 & 15 & 76 & 62 & 4 & 15 & 77 & 62 & 4 & 15 & 77 & 62 & 19 & 80 \\
\hline & & 85th & 0.487 & & 0.708 & 0.516 & 2.140 & & 2.038 & 2.219 & 0.373 & & 0.379 & 0.376 & 480.9 & 393.9 & 469.4 \\
\hline & & Min. & 0.126 & -0.022 & 0.186 & 0.126 & 1.167 & 0.286 & 0.820 & 0.820 & -0.058 & -0.064 & 0.033 & -0.058 & 229.2 & 73.2 & 168.3 \\
\hline Woodroffe Ave & Sample & Max. & 0.744 & 0.256 & 1.011 & 0.785 & 2.946 & 1.283 & 2.289 & 2.946 & 0.657 & 0.109 & 0.512 & 0.657 & 636.6 & 441.5 & 636.6 \\
\hline (W-NS) & & Mean & 0.352 & 0.100 & 0.459 & 0.363 & 1.754 & 0.915 & 1.695 & 1.746 & 0.255 & 0.040 & 0.293 & 0.266 & 413.2 & 294.9 & 388.5 \\
\hline & & SD & 0.14 & 0.12 & 0.24 & 0.15 & 0.41 & 0.44 & 0.37 & 0.42 & 0.13 & 0.08 & 0.13 & 0.13 & 76.50 & 100.19 & 89.90 \\
\hline & & 85 th & 0.498 & & 0.719 & 0.522 & 2.184 & & 2.097 & 2.177 & 0.394 & & 0.437 & 0.405 & 492.5 & 401.8 & 481.6 \\
\hline & Population & 95th & 0.584 & & 0.884 & 0.616 & 2.436 & & 2,353 & $\mathbf{2 . 4 3 0}$ & 0.476 & & 0.529 & 0.487 & 539.1 & 468.6 & 536.4 \\
\hline & & $\mathbf{N}$ & 82 & 3 & 32 & 113 & 82 & 3 & 32 & 114 & 81 & 3 & 32 & 113 & 94 & 41 & 135 \\
\hline & & 85th & 0.447 & & 0.684 & 0.509 & 2.611 & & 2.658 & 2.706 & 0.490 & & 0.527 & 0.510 & 555.7 & 453.4 & 543.8 \\
\hline & Sample & Min. & 0.029 & -0.116 & 0.107 & 0.029 & 1.127 & 1.135 & 1.650 & 1.127 & -0.051 & 0.018 & -0.056 & -0.056 & 208.4 & 126.7 & 126.7 \\
\hline Greenbank Rd & Sample & Max. & 0.611 & 0.074 & 0.851 & 0.790 & 3.381 & 2.241 & 3.213 & $\mathbf{3 . 3 8 1}$ & 0.721 & 0.321 & 0.656 & 0.721 & 587.5 & 553.3 & 587.5 \\
\hline (E-NS) & & Mean & 0.328 & 0.007 & 0.471 & 0.363 & 2.194 & 1.687 & 2.216 & 2.206 & 0.320 & 0.196 & 0.309 & 0.320 & 428.5 & 340.2 & 404.3 \\
\hline & & SD & 0.12 & 0.11 & 0.18 & 0.15 & 0.46 & 0.55 & 0.44 & 0.46 & 0.17 & 0.16 & 0.19 & 0.17 & 107.98 & 108.46 & 116.37 \\
\hline & Ponulation & 85th & 0.454 & & 0.654 & 0.517 & 2.668 & & 2.673 & 2.686 & 0.494 & & 0.501 & 0.499 & 540.4 & 452.6 & 524.8 \\
\hline & Population & 95th & 0.528 & & 0.762 & 0.607 & 2.947 & & 2.942 & 2.968 & 0.596 & & 0.614 & 0.605 & 606.1 & 518.6 & 595.7 \\
\hline & & $\mathbf{N}$ & 11 & 8 & 76 & 87 & 11 & 8 & 77 & 88 & 11 & 8 & 76 & 87 & 11 & 105 & 116 \\
\hline & & 85 th & 0.327 & & 0.450 & 0.431 & 1.699 & & 1.704 & 1.708 & 0.277 & & 0.348 & 0.349 & 304.5 & 271.3 & 283.9 \\
\hline & Sample & Min. & -0.237 & -0.584 & -0.253 & -0.253 & 0.867 & 0.568 & 0.559 & 0.559 & -0.195 & -0.181 & -0.421 & -0.421 & 192.1 & 25.9 & 25.9 \\
\hline Eagleson Rd & & Max. & 0.466 & 1.116 & 0.777 & 0.777 & 2.237 & 1.761 & 2.958 & 2.958 & 0.381 & 0.670 & 0.590 & 0.590 & 321.7 & 334.8 & 334.8 \\
\hline$(\mathrm{W}-\mathrm{NS})$ & & Mean & 0.146 & 0.020 & 0.259 & 0.244 & 1.427 & 1.008 & 1.416 & 1.432 & 0.108 & 0.010 & 0.112 & 0.111 & 279.7 & 200.1 & 207.6 \\
\hline & & SD & 0.22 & 0.54 & 0.20 & 0.20 & 0.40 & 0.41 & 0.52 & 0.52 & 0.17 & 0.28 & 0.20 & 0.20 & 35.56 & 71.11 & 72.33 \\
\hline & & 85th & 0.384 & & 0.465 & 0.455 & 1.865 & & 1.959 & 1.968 & 0.296 & & 0.323 & 0.319 & 318.6 & 273.7 & 282.5 \\
\hline & Population & 95th & 0.541 & & 0.586 & 0.579 & 2.153 & & 2.279 & 2.283 & 0.421 & & 0.447 & 0.442 & 344.2 & 317.0 & 326.6 \\
\hline
\end{tabular}


Table C-4 (b): DESCRIPTIVE SUMMARY FOR DECELERATION RATES AND EFFECTIVE DECELERATION DISTANCE ON EXTENDED SCLs (PC Only)

\begin{tabular}{|c|c|c|c|c|c|c|c|c|c|c|c|c|c|c|c|c|c|}
\hline \multirow{4}{*}{ SCL Name } & \multirow{4}{*}{\multicolumn{2}{|c|}{ Statistics }} & \multicolumn{12}{|c|}{ Deceleration Rate $\left(\mathrm{m} / \mathrm{s}^{2}\right)$} & \multirow{2}{*}{\multicolumn{3}{|c|}{ Deceleration Distance (m) }} \\
\hline & & & \multicolumn{4}{|c|}{ Overall } & \multicolumn{4}{|c|}{ Maximum } & \multicolumn{4}{|c|}{ Mean } & & & \\
\hline & & & \multirow{2}{*}{\multicolumn{2}{|c|}{\begin{tabular}{l|l}
\multicolumn{2}{c}{ Occupying SCL } \\
Depart & Merged
\end{tabular}}} & \multirow[t]{2}{*}{ Diverging } & \multirow[t]{2}{*}{ All } & \multicolumn{2}{|c|}{ Occupying SCL } & \multirow[t]{2}{*}{ Diverging } & \multirow[t]{2}{*}{ All } & \multicolumn{2}{|c|}{ Occupying SCL } & \multirow{2}{*}{ Diverging } & \multirow{2}{*}{$\overline{\text { All }}$} & \multirow[t]{2}{*}{ SCL } & \multirow[t]{2}{*}{ Diverging } & \multirow{2}{*}{ All } \\
\hline & & & & & & & Depart & Merged & & & Depart & Merged & & & & & \\
\hline & & $\mathrm{N}$ & 24 & & 31 & 55 & 25 & 1 & 31 & 56 & 24 & 1 & 31 & $\frac{56}{0.348}$ & $\frac{25}{4126}$ & $\frac{42}{3943}$ & $\frac{67}{411.8}$ \\
\hline & Sample & 85th & $\begin{array}{l}0.235 \\
-0.080\end{array}$ & 0.350 & $\begin{array}{l}0.370 \\
0.005\end{array}$ & $\begin{array}{r}0.333 \\
-0.080\end{array}$ & $\frac{1.786}{1.114}$ & 1.124 & $\begin{array}{l}1.688 \\
0.843\end{array}$ & $\begin{array}{l}1.696 \\
0.843\end{array}$ & $\begin{array}{l}0.264 \\
-0.090\end{array}$ & 0.330 & $\begin{array}{l}0.366 \\
-0.003\end{array}$ & $\begin{array}{r}0.348 \\
-0.090\end{array}$ & $\begin{array}{l}4.42 .0 \\
158.3 \\
\end{array}$ & 130.5 & $\begin{array}{l}411.8 \\
1130.5 \\
\end{array}$ \\
\hline St. Laurent Blvd & Dimpic & Max. & 0.497 & 0.350 & 0.636 & 0.636 & 2.540 & 1.124 & 2.276 & 2.540 & 0.446 & 0.330 & 0.615 & 0.706 & 439.8 & 442.9 & 442.9 \\
\hline (W-NS) & & Mean & 0.174 & 0.350 & 0.240 & 0.212 & 1.505 & 1.124 & 1.433 & 1.487 & 0.171 & 0.330 & 0.236 & 0.217 & 327.18 & 315.8 & 320.1 \\
\hline & & SD & 0.12 & & 0.16 & 0.15 & 0.45 & & 0.35 & 0.41 & 0.12 & & 0.17 & 0.17 & 85.50 & 82.53 & 83.18 \\
\hline & Population & 85th & 0.306 & & 0.408 & 0.368 & 1.982 & & 1.799 & 1.911 & 0.300 & & 0.407 & 0.388 & 417.7 & 401.3 & 406.2 \\
\hline & & 95th & 0.387 & & 0.507 & 0.459 & 2.276 & & 2.014 & 2.159 & 0.380 & & 0.508 & 0.489 & 473.5 & 451.6 & 456.9 \\
\hline & & $\mathrm{N}$ & 33 & 4 & 4 & 37 & 33 & 4 & 4 & 37 & 33 & 4 & 4 & 37 & 67 & 22 & 89 \\
\hline & & 85th & 0.556 & & 0.565 & 0.562 & 1.688 & & 1.779 & 1.688 & 0.421 & & 0.508 & 0.429 & 287.8 & 253.9 & 285.3 \\
\hline & Sample & Min..... & -0.033 & -0.318 & 0.059 & -0.033 & 1.102 & 0.565 & 1.119 & 1.102 & -0.063 & -0.212 & 0.022 & -0.063 & 92.8 & 26.8 & 26.8 \\
\hline Vanier Pkwy & & Max. & 0.851 & 0.295 & 0.582 & 0.851 & 1.832 & 1.675 & 1.862 & 1.862 & 0.529 & 0.182 & 0.567 & 0.567 & 328.3 & 294.1 & 328.3 \\
\hline (W-NS) & & Mean & 0.351 & 0.089 & 0.375 & 0.354 & 1.506 & 1.224 & 1.582 & 1.528 & 0.230 & 0.003 & 0.326 & 0.240 & 226.9 & 167.6 & 212.3 \\
\hline & & SD & 0.20 & 0.28 & 0.24 & 0.20 & 0.23 & 0.47 & 0.32 & 0.23 & 0.17 & 0.18 & 0.23 & 0.18 & 58.02 & 81.92 & 69.20 \\
\hline & Population & $85 \mathrm{th}$ & 0.561 & & 0.677 & 0.565 & 1.744 & & 1.983 & 1.767 & 0.408 & & 0.619 & 0.425 & 287.1 & 254.7 & 284.0 \\
\hline & & 95th & 0.685 & & 0.943 & 0.689 & 1.883 & & 2.337 & 1.907 & 0.513 & & 0.877 & 0.534 & 322.4 & 308.6 & 326.1 \\
\hline & & $\mathbf{N}$ & 61 & 4 & 15 & 75 & 61 & 4 & 15 & 76 & 61 & 4 & 15 & 76 & 61 & 19 & 79 \\
\hline & & 85 th & 0.490 & & 0.708 & 0.519 & 2.000 & & 2.038 & 2.064 & 0.373 & & 0.379 & 0.376 & 481.3 & 393.9 & 469.3 \\
\hline & Sample & Min. & 0.126 & -0.022 & 0.186 & 0.126 & 1.167 & 0.286 & 0.820 & 0.820 & -0.058 & -0.064 & 0.033 & -0.058 & 229.2 & 73.2 & 168.3 \\
\hline Woodroffe Ave & & Max. & 0.744 & 0.256 & 1.011 & 0.785 & 2.862 & 1.283 & 2.289 & 2.862 & 0.657 & 0.109 & 0.512 & 0.657 & 636.6 & 441.5 & 636.6 \\
\hline W & & Mean & 0.355 & 0.100 & 0.459 & 0.366 & 1.733 & 0.915 & 1.695 & 1.728 & 0.255 & 0.040 & 0.293 & 0.267 & 412.3 & 294.9 & 387.5 \\
\hline & & SD & 0.14 & 0.12 & 0.24 & 0.15 & 0.39 & 0.44 & 0.37 & 0.39 & 0.14 & 0.08 & 0.13 & 0.14 & 76.80 & 100.19 & 90.01 \\
\hline & Population & 85th & 0.500 & & 0.719 & 0.524 & 2.133 & & 2.097 & 2.134 & 0.396 & & 0.437 & 0.407 & 491.9 & 401.8 & 480.7 \\
\hline & & 95th & 0.585 & & 0.884 & 0.618 & 2.367 & & 2.353 & 2.372 & 0.478 & & 0.529 & 0.489 & 538.6 & 468.6 & 535.5 \\
\hline & & $\mathbf{N}$ & 69 & 3 & 32 & 100 & 69 & 3 & 32 & 101 & 69 & 3 & 32 & 101 & 81 & 40 & 121 \\
\hline & & 85 th & 0.449 & & 0.684 & 0.510 & 2.612 & & 2.658 & 2.743 & 0.518 & & 0.527 & 0.525 & 557.3 & 452.4 & 540.2 \\
\hline & Sample & Min. & 0.029 & -0.116 & 0.107 & 0.029 & 1.127 & 1.135 & 1.650 & 1.127 & -0.051 & 0.018 & -0.056 & -0.056 & 214.2 & 126.7 & 126.7 \\
\hline Greenbank Rd & & Max. & 0.611 & 0.074 & 0.851 & 0.790 & 3.381 & 2.241 & 3.213 & 3.3 & 0.721 & 0.321 & 0.656 & 0.721 & 587.5 & 553.3 & $\mathbf{5 8 7 . 5}$ \\
\hline (E-) & & Mean & 0.334 & 0.007 & 0.471 & 0.3 & 2.202 & 1.687 & 2.216 & 2.206 & 0.339 & 0.196 & 0.309 & & & 337.0 & 401.6 \\
\hline & & SD & 0.12 & 0.11 & 0.18 & 0.15 & 0.46 & 0.55 & 0.44 & 0.47 & 0.17 & 0.16 & 0.19 & 0.17 & 107.01 & 107.81 & 116.61 \\
\hline & Population & 85 th & 0.459 & & 0.654 & 0.525 & 2.679 & & 2.673 & 2.690 & 0.512 & & 0.501 & 0.513 & 541.1 & 448.7 & 522.4 \\
\hline & & 95th & 0.532 & & 0.762 & 0.615 & 2.960 & & 2.942 & 2.975 & 0.613 & & 0.614 & 0.618 & 606.3 & 514.3 & 593.5 \\
\hline & & $\mathbf{N}$ & 8 & 8 & 70 & 78 & 8 & 8 & 71 & 79 & 8 & 8 & 70 & 78 & 8 & 99 & 107 \\
\hline & & 85th & & & 0.474 & 0.43 & & & 1.705 & 1.710 & & & 0.363 & 0.349 & & 265.4 & 278.2 \\
\hline & Sample & Min. & -0.237 & -0.584 & -0.253 & -0.253 & 0.867 & 0.568 & 0.559 & 0.559 & -0.195 & -0.181 & -0.357 & -0.357 & 261.5 & 25.9 & 25.9 \\
\hline Eagleson Rd & & Max... & 0.240 & 1.116 & 0.777 & 0.77 & 2.237 & 1.761 & 2.958 & 2.958 & 0.349 & 0.670 & 0.590 & 0.590 & 305.7 & 297.0 & 305.7 \\
\hline (W-NS) & & Mean & 0.061 & 0.020 & 0.267 & 0.244 & 1.398 & 1.008 & 1.437 & 1.438 & 0.065 & 0.010 & & 0.1 & 289.4 & 195.4 & 202.4 \\
\hline & & SD & 0.19 & 0.54 & 0.20 & 0.21 & 0.44 & 0.41 & 0.54 & 0.53 & 0.17 & 0.28 & 0.20 & 0.20 & 14.26 & 68.77 & 70.73 \\
\hline & Population & 85th & & & 0.478 & 0.463 & & & 1.993 & 1.989 & & & 0.329 & 0.322 & & 266.6 & 275.7 \\
\hline & & 95th & & & 0.602 & 0.592 & & & 2.320 & 2.313 & & & 0.451 & 0.443 & & 308.5 & 318.8 \\
\hline
\end{tabular}


Table C-4 (c): DESCRIPTIVE SUMMARY FOR DECELERATION RATES AND EFFECTIVE DECELERATION DISTANCE ON WOODROFFE (E-NS) SCL (COMBINED PC \& HV)

\begin{tabular}{|c|c|c|c|c|c|c|c|c|c|c|c|c|c|c|c|c|c|}
\hline \multirow{4}{*}{$\begin{array}{c}\text { Section } \\
\text { (Depart) }\end{array}$} & \multirow{4}{*}{\multicolumn{2}{|c|}{ Statistics }} & \multicolumn{12}{|c|}{ Deceleration Rate $\left(\mathrm{m} / \mathrm{s}^{2}\right)$} & \multirow{2}{*}{\multicolumn{3}{|c|}{ Deceleration Distance $(\mathrm{m})$}} \\
\hline & & & \multicolumn{4}{|c|}{ Overall } & \multicolumn{4}{|c|}{ Maximum } & \multicolumn{4}{|c|}{ Mean } & & & \\
\hline & & & \multirow{2}{*}{\multicolumn{2}{|c|}{ Occupying SCL }} & \multirow{2}{*}{ Diverging } & \multirow[b]{2}{*}{ All } & \multicolumn{2}{|c|}{ Occupying SCL } & \multirow{2}{*}{ Diverging } & \multirow{2}{*}{ All } & \multicolumn{2}{|c|}{ Occupying SCL } & \multirow{3}{*}{$\frac{\text { Diverging }}{28}$} & \multirow{3}{*}{$\frac{\text { All }}{42}$} & \multirow{3}{*}{$\frac{\mathrm{SCL}}{14}$} & \multirow{3}{*}{$\begin{array}{c}\text { Diverging } \\
28 \\
\end{array}$} & \multirow{3}{*}{ All } \\
\hline & & & Depart & & & & Depart & Merged & & & Depart & Merged & & & & & \\
\hline \multirow{8}{*}{$\begin{array}{l}\text { Before North-Exit } \\
\text { (North) }\end{array}$} & \multirow{6}{*}{ Sample } & $\mathbf{N}$ & 14 & 6 & 28 & 42 & 14 & 6 & 28 & 42 & 14 & 6 & & & & & \\
\hline & & 85 th & 0.975 & & 1.014 & 1.014 & 3.065 & & 2.836 & 2.846 & 0.747 & & 0.908 & 0.836 & 258.5 & 239.1 & 255.5 \\
\hline & & Min. & 0.123 & -0.251 & 0.417 & 0.123 & 1.672 & 1.116 & 1.700 & 1.672 & 0.000 & -0.219 & 0.183 & 0.000 & 120.1 & 29.8 & 29.8 \\
\hline & & Max. & 1.101 & 0.125 & 1.549 & 1.549 & 3.247 & 2.324 & 3.510 & 3.510 & 0.808 & 0.176 & 1.193 & 1.193 & 326.1 & 308.9 & 326.1 \\
\hline & & Mean & 0.610 & -0.024 & 0.798 & 0.735 & 2.494 & 1.508 & 2.471 & 2.479 & 0.471 & -0.001 & 0.641 & 0.585 & 195.3 & 156.9 & 169.7 \\
\hline & & SD & 0.32 & 0.15 & 0.25 & 0.29 & 0.49 & 0.48 & 0.48 & 0.48 & 0.26 & 0.17 & 0.22 & 0.24 & 58.05 & 70.95 & 68.70 \\
\hline & \multirow{2}{*}{ Population } & 85th & 0.952 & & 1.066 & 1.032 & 3.021 & & 2.980 & 2.974 & 0.748 & & 0.876 & 0.838 & 257.951 & 231.9 & 240.9 \\
\hline & & 95th & 1.172 & & 1.229 & 1.207 & 3.359 & & 3.292 & 3.265 & 0.925 & & 1.019 & 0.987 & 298.124 & 277.7 & 282.7 \\
\hline & & $N$ & 2 & & 55 & 76 & & & 55 & 76 & 2 & 1 & 55 & 76 & 21 & 55 & 76 \\
\hline & & 85 th & 0.4. & & 0.384 & 0.399 & 2.8 & & 2.407 & 2.594 & & 22 & 0.384 & 0.384 & 434.9 & 463.4 & 455.0 \\
\hline & & Min. & & & 0.017 & -0.015 & & & 0.828 & 0.828 & & 215 & -0.171 & -0.215 & 256.2 & 242.8 & 242.8 \\
\hline Before North-Exit & Sample & Max. & 0.2 & & 0.685 & 0.711 & & & 3.405 & 3.405 & 0. & 88 & 0.611 & 0.611 & 513.3 & 576.8 & 576.8 \\
\hline (South) & & Mean & 0.2 & & 0.291 & 0.283 & 2. & & 2.100 & 2.083 & 0. & 74 & 0.233 & 0.217 & 347.0 & 368.9 & 362.8 \\
\hline & & SD & 0. & & 0.15 & 0.16 & & 60 & 0.51 & 0.53 & & 20 & 0.15 & 0.17 & 74.07 & 86.47 & 83.32 \\
\hline & & 85th & 0.4. & & 0.444 & 0.453 & 2. & 77 & 2.628 & 2.635 & 0. & 89 & 0.386 & 0.388 & 425.8 & 458.4 & 449.2 \\
\hline & Poputartion & 95th & 0.6 & & 0.534 & 0.553 & & 74 & 2.938 & 2.959 & & 23 & 0.477 & 0.489 & 474.8 & 511.1 & 499.9 \\
\hline & & $\mathrm{N}$ & 4 & & 66 & 114 & 5 & 3 & 66 & 118 & 5 & 3 & 65 & 118 & 51 & 66 & 118 \\
\hline & & 85th & & & 0.882 & 0.706 & & 92 & 2.569 & 2.593 & & 14 & 0.697 & 0.636 & 233.6 & 234.1 & 234.9 \\
\hline & Sample & Min. & -0 . & & -0.850 & -0.435 & 0. & 48 & 1.043 & 1.043 & -0 . & 309 & -0.519 & -0.519 & 126.1 & 81.7 & 81.7 \\
\hline After North-Exit & & Max. & 0.5 & & 1.576 & 1.342 & 2.8 & 40 & 2.897 & 2.897 & 0. & 64 & 1.175 & 1.175 & 306.5 & 301.8 & 335.6 \\
\hline South 2 \& & & Mean & 0. & & 0.479 & 0.409 & & 154 & 2.077 & 2.079 & 0. & 102 & 0.358 & 0.333 & 213.1 & 186.2 & 199.1 \\
\hline & & SD & 0. & & 0.44 & 0.31 & 0. & 52 & 0.48 & 0.48 & & 26 & 0.34 & 0.31 & 32.62 & 48.18 & 45.64 \\
\hline & Population & 85th & 0.6 & & 0.932 & 0.728 & 2. & 190 & 2.579 & 2.576 & 0. & 71 & 0.715 & 0.653 & 246.9 & 236.1 & 246.4 \\
\hline & Popurantion & 95th & 0.7 & & 1.198 & 0.916 & 2. & 06 & 2.874 & 2.868 & & 29 & 0.925 & 0.841 & 266.8 & 265.5 & 274.2 \\
\hline & & $\mathbf{N}$ & 7 & 2 & 116 & 186 & 7 & 4 & 121 & 195 & 7 & 4 & 119 & 192 & 72 & 132 & 204 \\
\hline & & 85 th & 0.5 & 35 & 0.617 & 0.607 & 2.8 & 49 & 2.556 & 2.600 & 0. & 99 & 0.588 & 0.530 & 328.2 & 386.0 & 356.1 \\
\hline & Sample & Min. & & 183 & -0.435 & -0.205 & & 48 & 0.828 & 0.548 & & 309 & -0.519 & -0.438 & 126.1 & 81.7 & 81.7 \\
\hline Total & sample & Max. & 0.5 & & 1.140 & 1.019 & 3. & 08 & 3.405 & 3.405 & 0. & 64 & 1.053 & 1.053 & 443.7 & 576.8 & 546.5 \\
\hline (South) & & Mean & 0.3 & 40 & 0.367 & 0.357 & 2.8 & 49 & 2.087 & 2.073 & 0. & 65 & 0.294 & 0.287 & 248.3 & 265.5 & 258.7 \\
\hline & & SD & 0. & & 0.27 & 0.24 & 0. & 54 & 0.49 & 0.51 & 0. & 25 & 0.27 & 0.25 & 68.15 & 111.95 & 96.87 \\
\hline & who & 85 th & 0.5 & 69 & 0.648 & 0.606 & & 07 & 2.599 & 2.602 & 0. & 24 & 0.571 & 0.551 & 318.9 & 381.5 & 359.1 \\
\hline & Fopuliantion & 95th & 0.7 & 04 & 0.813 & 0.753 & 2.5 & 35 & 2.900 & 2.912 & 0. & 77 & 0.734 & 0.706 & 360.4 & 449.7 & 418.1 \\
\hline
\end{tabular}


Table C-4 (d): DESCRIPTIVE SUMMARY FOR DECELERATION RATES AND EFFECTIVE DECELERATION DISTANCE ON WOODROFFE (E-NS) SCL (PC ONLY)

\begin{tabular}{|c|c|c|c|c|c|c|c|c|c|c|c|c|c|c|c|c|c|}
\hline \multirow{4}{*}{$\begin{array}{c}\text { Section } \\
\text { (Depart) }\end{array}$} & \multirow{4}{*}{\multicolumn{2}{|c|}{ Statistics }} & \multicolumn{12}{|c|}{ Deceleration Rate $\left(\mathrm{m} / \mathrm{s}^{2}\right)$} & \multirow{2}{*}{\multicolumn{3}{|c|}{ Deceleration Distance (m) }} \\
\hline & & & \multicolumn{4}{|c|}{ Overall } & \multicolumn{4}{|c|}{ Maximum } & \multicolumn{4}{|c|}{ Mean } & & & \\
\hline & & & \multirow{2}{*}{\multicolumn{2}{|c|}{ Occupying SCL }} & \multirow{2}{*}{ Diverging } & \multirow{2}{*}{ All } & \multicolumn{2}{|c|}{ Occupying SCL } & \multirow{2}{*}{ Diverging } & \multirow{2}{*}{ All } & \multicolumn{2}{|c|}{ Occupying SCL } & \multirow{3}{*}{$\begin{array}{c}\text { Diverging } \\
28\end{array}$} & \multirow{2}{*}{ All } & \multirow{2}{*}{$\mathrm{SCL}$} & \multirow{2}{*}{ Diverging } & \multirow{2}{*}{ All } \\
\hline & & & & & & & Depart & Merged & & & Depart & Merged & & & & & \\
\hline \multirow{8}{*}{$\begin{array}{l}\text { Before North-Exit } \\
\text { (North) }\end{array}$} & \multirow{6}{*}{ Sample } & $\mathbf{N}$ & 12 & 5 & 28 & 40 & 12 & 5 & 28 & 40 & 12 & 5 & & 40 & 12 & 28 & 40 \\
\hline & & 85 th & 0.958 & & 1.014 & 1.016 & 3.075 & & 2.836 & 2.864 & 0.735 & & 0.908 & 0.851 & 227.6 & 239.1 & 242.6 \\
\hline & & Min. & 0.123 & -0.251 & 0.417 & 0.123 & 1.672 & 1.116 & 1.700 & 1.672 & 0.000 & -0.219 & 0.183 & 0.000 & 120.1 & 29.8 & 29.8 \\
\hline & & Max. & 1.101 & 0.125 & 1.549 & 1.549 & 3.247 & 2.324 & 3.510 & 3.510 & 0.793 & 0.176 & 1.193 & 1.193 & 326.1 & 308.9 & 326.1 \\
\hline & & Mean & 0.604 & -0.034 & 0.798 & 0.739 & 2.571 & 1.585 & 2.471 & 2.501 & 0.455 & -0.002 & 0.641 & 0.586 & 187.8 & 156.9 & 166.1 \\
\hline & & SD & 0.32 & 0.17 & 0.25 & 0.28 & 0.47 & 0.50 & 0.48 & 0.47 & 0.25 & 0.19 & 0.22 & 0.24 & 58.83 & 70.95 & 68.31 \\
\hline & \multirow{2}{*}{ Population } & 85 th & 0.947 & & 1.066 & 1.034 & 3.079 & & 2.980 & 2.992 & 0.733 & & 0.876 & 0.839 & 251.8 & 231.9 & 236.9 \\
\hline & & 95 th & 1.170 & & 1.229 & 1.207 & 3.409 & & 3.292 & 3.280 & 0.913 & & 1.019 & 0.988 & 293.4 & 277.7 & 278.5 \\
\hline & & $\mathbf{N}$ & & 8 & 55 & 73 & & & 55 & 73 & 1 & & 55 & 73 & 18 & 55 & 73 \\
\hline & & 85 th & & 50 & 0.384 & 0.405 & & & 2.407 & 2.641 & 0.3 & 70 & 0.384 & 0.384 & 415.9 & 463.4 & 457.9 \\
\hline & & Min. & & 04 & 0.017 & 0.004 & & & 0.828 & 0.828 & -0. & 15 & -0.171 & -0.215 & 256.2 & 242.8 & 242.8 \\
\hline & Sample & Max. & & 11 & 0.685 & 0.711 & & & 3.405 & 3.405 & 0.5 & 88 & 0.611 & 0.611 & 513.3 & 576.8 & 576.8 \\
\hline (South) & & Mean & & & 0.291 & 0.289 & & & 2.100 & 2.107 & 0.1 & 84 & 0.233 & 0.221 & 344.0 & 368.9 & 362.7 \\
\hline & & SD & & 20 & 0.15 & 0.16 & & & 0.51 & 0.52 & 0. & 21 & 0.15 & 0.16 & 73.01 & 86.47 & 83.56 \\
\hline & & 85 th & & 00 & 0.444 & 0.457 & & 24 & 2.628 & 2.644 & 0.4 & 07 & 0.386 & 0.392 & 422.0 & 458.4 & 449.3 \\
\hline & Population & 95th & & 35 & 0.534 & 0.555 & & & 2.938 & 2.959 & 0.5 & 47 & 0.477 & 0.492 & 471.0 & 511.1 & 500.2 \\
\hline & & $\mathbf{N}$ & & & 66 & 112 & & & 66 & 116 & 5 & & 65 & 116 & 48 & 66 & 116 \\
\hline & & 85 th & & & 0.882 & 0.707 & & 11 & 2.569 & 2.602 & 0.5 & & 0.697 & 0.640 & 232.4 & 234.1 & 235.0 \\
\hline & & Min. & & 83 & -0.850 & -0.435 & & 48 & 1.043 & 1.043 & -0. & 309 & -0.519 & -0.519 & 149.5 & 81.7 & 81.7 \\
\hline After North-Exit & Sample & Max. & & 13 & 1.576 & 1.342 & & 40 & 2.897 & 2.897 & 0.9 & 64 & 1.175 & 1.175 & 283.6 & 301.8 & 335.6 \\
\hline (South) & & Mean & & 77 & 0.479 & 0.411 & & 70 & 2.077 & 2.087 & 0.3 & 08 & 0.358 & 0.336 & 214.6 & 186.2 & 200.3 \\
\hline & & SD & & & 0.44 & 0.31 & & 52 & 0.48 & 0.48 & 0. & & 0.34 & 0.31 & 25.64 & 48.18 & 45.13 \\
\hline & Ponulation & 85th & & 10 & 0.932 & 0.733 & & 09 & 2.579 & 2.584 & 0. & 79 & 0.715 & 0.658 & 241.1 & 236.1 & 247.0 \\
\hline & Population & 95th & & 47 & 1.198 & 0.922 & & 27 & 2.874 & 2.877 & 0.7 & 39 & 0.925 & 0.847 & 256.8 & 265.5 & 274.5 \\
\hline & & $\mathbf{N}$ & & & 116 & 181 & & & 121 & 190 & 6 & & 119 & 187 & 66 & 131 & 199 \\
\hline & & 85 th & & 56 & 0.617 & 0.608 & & 36 & 2.556 & 2.607 & 0. & 10 & 0.588 & 0.532 & 315.5 & 387.1 & 354.2 \\
\hline & & Min. & & 183 & -0.435 & -0.205 & & 48 & 0.828 & 0.548 & -0. & 309 & -0.519 & -0.438 & 149.5 & 81.7 & 81.7 \\
\hline & Sample & Max. & & 13 & 1.140 & 1.019 & & 08 & 3.405 & 3.405 & 0. & 64 & 1.053 & 1.053 & 400.4 & 576.8 & 546.5 \\
\hline (Soutb) & & Mean & & 53 & 0.367 & 0.362 & & 85 & 2.087 & 2.086 & 0.2 & 76 & 0.294 & 0.291 & 243.7 & 266.3 & 258.4 \\
\hline & & SD & & 22 & 0.27 & 0.24 & & 53 & 0.49 & 0.51 & 0 & 25 & 0.27 & 0.26 & 56.44 & 112.01 & 95.89 \\
\hline & & 85th & & 82 & 0.648 & 0.612 & & 31 & 2.599 & 2.610 & 0.5 & 38 & 0.571 & 0.557 & 302.2 & 382.4 & 357.8 \\
\hline & Population & 95th & & 17 & 0.813 & 0.758 & & 53 & 2.900 & 2.917 & 0.6 & 93 & 0.734 & 0.713 & 336.5 & 450.6 & 416.1 \\
\hline
\end{tabular}


Table C-5: DESCRIPTIVE SUMMARY FOR MEAN SPEEDS ON RIGHT LANES ADJACENT TO LIMITED SCLS

\begin{tabular}{|c|c|c|c|c|c|c|c|c|c|c|}
\hline \multirow{3}{*}{ SCL Name } & \multirow{3}{*}{\multicolumn{2}{|c|}{ Statistics }} & \multicolumn{4}{|c|}{ Combined PC \& HV } & \multicolumn{4}{|c|}{ PC Only } \\
\hline & & & \multicolumn{8}{|c|}{ Mean Speed km/h } \\
\hline & & & Through & Diverged & ML & All & Through & Diverged & ML & All \\
\hline \multirow{8}{*}{$\begin{array}{l}\text { Bronson Ave } \\
\quad(\mathrm{W}-\mathrm{NS})\end{array}$} & \multirow{6}{*}{ Sample } & $\mathbf{N}$ & 78 & 39 & 2 & 119 & 76 & 31 & 2 & 109 \\
\hline & & 85th & 109.7 & 98.3 & & 107.0 & 109.8 & 98.3 & & 108.4 \\
\hline & & Min. & 72.8 & 70.9 & 100.5 & 70.9 & 72.8 & 73.9 & 100.5 & 72.8 \\
\hline & & Max. & 125.9 & 114.5 & 110.8 & 125.9 & 125.9 & 114.5 & 110.8 & 125.9 \\
\hline & & Mean & 99.6 & 90.3 & 105.7 & 96.6 & 99.6 & 92.5 & 105.7 & 97.7 \\
\hline & & SD & 9.84 & 9.27 & 7.27 & 10.58 & 9.97 & 7.75 & 7.27 & 9.88 \\
\hline & \multirow{2}{*}{ Population } & 85th & 109.8 & 99.9 & & 107.6 & 109.9 & 100.5 & & 107.9 \\
\hline & & 95th & 115.8 & 105.5 & & 114.0 & 116.0 & 105.2 & & 113.9 \\
\hline \multirow{8}{*}{$\begin{array}{l}\text { Parkdale Ave } \\
\text { (W-NS) }\end{array}$} & \multirow{6}{*}{ Sample } & $\mathbf{N}$ & 81 & 45 & 1 & 127 & 72 & 45 & 1 & 118 \\
\hline & & 85th & 109.1 & 98.9 & & 107.2 & 110.8 & 98.9 & & 107.4 \\
\hline & & Min. & 75.1 & 72.6 & 100.4 & 72.6 & 75.1 & 72.6 & 100.4 & 72.6 \\
\hline & & Max. & 119.3 & 115.4 & 100.4 & 119.3 & 119.3 & 115.4 & 100.4 & 119.3 \\
\hline & & Mean & 99.6 & 90.5 & 100.4 & 96.4 & 100.2 & 90.5 & 100.4 & 96.5 \\
\hline & & SD & 9.28 & 8.87 & & 10.07 & 9.39 & 8.87 & & 10.28 \\
\hline & \multirow{2}{*}{ Population } & 85th & 109.2 & 99.7 & & 106.8 & 109.9 & 99.7 & & 107.1 \\
\hline & & 95th & 114.9 & 105.1 & & 113.0 & 115.7 & 105.1 & & 113.4 \\
\hline \multirow{8}{*}{$\begin{array}{l}\text { Prom Island Park Dr } \\
\qquad(\mathrm{E}-\mathrm{N})\end{array}$} & \multirow{6}{*}{ Sample } & $\mathbf{N}$ & 90 & 60 & 4 & 154 & 77 & 60 & 4 & 141 \\
\hline & & 85th & 101.2 & 90.8 & & 99.4 & 101.2 & 90.8 & & 99.4 \\
\hline & & Min. & 74.5 & 62.1 & 78.3 & 62.1 & 74.5 & 62.1 & 78.3 & 62.1 \\
\hline & & Max. & 111.5 & 97.7 & 101.2 & 111.5 & 111.5 & 97.7 & 101.2 & 111.5 \\
\hline & & Mean & 93.1 & 82.0 & 94.9 & 88.8 & 93.1 & 82.0 & 94.9 & 88.4 \\
\hline & & SD & 8.42 & 7.87 & 11.09 & 9.87 & 8.60 & 7.87 & 11.09 & 9.98 \\
\hline & \multirow{2}{*}{ Population } & 85th & 101.8 & 90.2 & & 99.1 & 102.0 & 90.2 & & 98.8 \\
\hline & & 95th & 107.0 & 95.0 & & 105.1 & 107.2 & 95.0 & & 104.8 \\
\hline & & $\mathbf{N}$ & 103 & 5 & & 108 & 90 & 4 & & 94 \\
\hline & & 85 th & 112.4 & & & 112.1 & 113.1 & & & 113.0 \\
\hline & Samole & Min. & 81.6 & 89.1 & & 81.6 & 90.9 & 89.1 & & 89.1 \\
\hline Moodie Dr & Sample & Max. & 126.9 & 104.9 & & 126.9 & 126.9 & 103.9 & & 126.9 \\
\hline (W-NS) & & Mean & 104.7 & 99.2 & & 104.5 & 105.9 & 97.8 & & 105.5 \\
\hline & & SD & 7.80 & 6.56 & & 7.81 & 7.27 & 6.60 & & 7.39 \\
\hline & Panulation & 85th & 112.8 & & & 112.6 & 113.4 & & & 113.2 \\
\hline & Population & 95th & 117.6 & & & 117.3 & 117.8 & & & 117.7 \\
\hline & & $\mathbf{N}$ & 73 & 50 & & 123 & 62 & 48 & & 110 \\
\hline & & 85th & 111.6 & 117.4 & & 114.1 & 112.2 & 117.5 & & 114.7 \\
\hline & Samole & Min. & 86.3 & 81.5 & & 81.5 & 86.3 & 81.5 & & 81.5 \\
\hline Terry Fox Dr & Sample & Max. & 122.0 & 124.9 & & 124.9 & 122.0 & 124.9 & & 124.9 \\
\hline (E-NS) & & Mean & 104.0 & 105.0 & & 104.4 & 104.8 & 105.2 & & 105.0 \\
\hline & & SD & 7.25 & 9.91 & & 8.41 & 7.27 & 10.08 & & 8.57 \\
\hline & Ponul & 85th & 111.5 & 115.3 & & 113.1 & 112.4 & 115.6 & & 113.9 \\
\hline & Population & 95th & 116.0 & 121.3 & & 118.3 & 116.8 & 121.8 & & 119.1 \\
\hline & & $\mathbf{N}$ & 104 & 3 & & 107 & 93 & 3 & & 96 \\
\hline & & 85th & 116.1 & & & 115.9 & 117.0 & & & 116.8 \\
\hline & Sample & Min. & 86.2 & 86.1 & & 86.1 & 86.2 & 86.1 & & 86.1 \\
\hline Terry Fox Dr & Sample & Max. & 130.8 & 111.7 & & 130.8 & 130.8 & 111.7 & & 130.8 \\
\hline (W-NS) & & Mean & 107.6 & 103.1 & & 107.5 & 108.1 & 103.1 & & 108.0 \\
\hline & & SD & 8.51 & 14.75 & & 8.66 & 8.63 & 14.75 & & 8.80 \\
\hline & Panulation & 85th & 116.4 & & & 116.4 & 117.1 & & & 117.1 \\
\hline & Population & 95th & 121.6 & & & 121.7 & 122.3 & & & 122.5 \\
\hline & & $\mathbf{N}$ & 106 & 19 & & 125 & 87 & 14 & & 101 \\
\hline & & 85th & 117.6 & 112.8 & & 117.3 & 118.6 & 114.4 & & 117.9 \\
\hline & Samnla & Min. & 87.1 & 89.2 & & 87.1 & 88.5 & 89.2 & & 88.5 \\
\hline Ch. Carp Rd & Sample & Max. & 127.4 & 117.3 & & 127.4 & 127.4 & 117.3 & & 127.4 \\
\hline (E-NS) & & Mean & 108.1 & 104.4 & & 107.5 & 109.8 & 106.0 & & 109.3 \\
\hline . & & SD & 9.02 & 8.03 & & 8.94 & 8.71 & 8.31 & & 8.71 \\
\hline & & 85th & 117.4 & 113.0 & & 116.8 & 118.8 & 114.9 & & 118.3 \\
\hline & Population & 95th & 122.9 & 118.4 & & 122.2 & 124.1 & 120.7 & & 123.6 \\
\hline
\end{tabular}


Table C-6: DESCRIPTIVE SUMMARY FOR MEAN SPEEDS ON RIGHT LANES ADJACENT TO EXTENDED SCLS

\begin{tabular}{|c|c|c|c|c|c|c|c|c|c|c|c|c|}
\hline \multirow{3}{*}{ SCL Name } & \multirow{3}{*}{\multicolumn{2}{|c|}{ Statistics }} & \multicolumn{5}{|c|}{ Combined PC \& HV } & \multicolumn{5}{|c|}{ PC Only } \\
\hline & & & \multicolumn{10}{|c|}{ Mean Speed km/h } \\
\hline & & & Through & Diverged & ML & Exit & All & Through & Diverged & ML & Exit & All \\
\hline \multirow{8}{*}{$\begin{array}{l}\text { St. Laurent Blvd } \\
\text { (W-NS) }\end{array}$} & \multirow{6}{*}{ Sample } & $\mathbf{N}$ & 52 & 10 & 5 & 22 & 89 & 49 & 10 & 5 & 21 & 85 \\
\hline & & 85th & 107.1 & 100.9 & & 105.5 & 105.7 & 107.5 & 100.9 & & 105.7 & 105.8 \\
\hline & & Min. & 73.5 & 89.3 & 79.1 & 85.2 & 73.5 & 82.9 & 89.3 & 79.1 & 85.2 & 79.1 \\
\hline & & Max. & 114.6 & 102.2 & 96.2 & 113.8 & 114.6 & 114.6 & 102.2 & 96.2 & 113.8 & 114.6 \\
\hline & & Mean & 96.6 & 96.6 & 88.4 & 97.3 & 96.3 & 97.5 & 96.6 & 88.4 & 97.8 & 96.9 \\
\hline & & SD & 8.87 & 4.12 & 7.01 & 8.22 & 8.34 & 8.16 & 4.12 & 7.01 & 8.05 & 7.90 \\
\hline & \multirow{2}{*}{ Population } & 85th & 105.8 & 101.1 & & 106.0 & 104.9 & 105.9 & 101.1 & & 106.3 & 105.1 \\
\hline & & 95th & 111.2 & 104.1 & & 111.4 & 110.0 & 110.9 & 104.1 & & 111.7 & 109.9 \\
\hline \multirow{8}{*}{$\begin{array}{c}\text { Vanier Pkwy } \\
\text { (W-NS) }\end{array}$} & \multirow{6}{*}{ Sample } & $\mathbf{N}$ & 90 & 6 & 7 & 24 & 125 & 76 & 6 & 6 & 24 & 110 \\
\hline & & 85th & 104.4 & & & 101.7 & 104.3 & 104.6 & & & 101.7 & 104.5 \\
\hline & & Min. & 78.4 & 93.8 & 87.1 & 75.2 & 75.2 & 83.6 & 93.8 & 87.1 & 75.2 & 75.2 \\
\hline & & Max. & 110.6 & 104.3 & 128.0 & 120.7 & 115.8 & 110.6 & 104.3 & 128.0 & 120.7 & 115.8 \\
\hline & & Mean & 96.3 & 97.9 & 100.1 & 91.9 & 95.3 & 97.3 & 97.9 & 100.9 & 91.9 & 95.9 \\
\hline & & SD & 6.90 & 4.35 & 14.11 & 11.19 & 7.65 & 6.51 & 4.35 & 15.28 & 11.19 & 7.66 \\
\hline & \multirow{2}{*}{ Population } & 85th & 103.4 & & & 103.7 & 103.2 & 104.1 & & & 103.7 & 103.8 \\
\hline & & 95th & 107.6 & & & 111.0 & 107.9 & 108.0 & & & 111.0 & 108.5 \\
\hline \multirow{8}{*}{$\begin{array}{l}\text { Woodroffe Ave } \\
\text { (E-NS) }\end{array}$} & \multirow{6}{*}{ Sample } & $\mathbf{N}$ & 94 & 11 & 4 & & 107 & 89 & 11 & 4 & & 103 \\
\hline & & 85th & 111.3 & 116.9 & & & 112.3 & 111.4 & 116.9 & & & 113.9 \\
\hline & & Min. & 87.3 & 72.5 & 108.6 & & 81.3 & 87.3 & 72.5 & 108.6 & & 81.3 \\
\hline & & Max. & 125.6 & 122.6 & 130.3 & & 125.6 & 125.6 & 122.6 & 130.3 & & 130.3 \\
\hline & & Mean & 102.5 & 99.8 & 118.0 & & 102.8 & 102.6 & 99.8 & 118.0 & & 103.2 \\
\hline & & SD & 7.65 & 15.28 & 9.55 & & 8.36 & 7.83 & 15.28 & 9.55 & & 8.91 \\
\hline & \multirow{2}{*}{ Population } & 85th & 110.4 & 116.5 & & & 111.5 & 110.8 & 116.5 & & & 112.5 \\
\hline & & 95th & 115.1 & 127.5 & & & 116.6 & 115.5 & 127.5 & & & 117.9 \\
\hline \multirow{8}{*}{$\begin{array}{l}\text { Woodroffe Ave } \\
\text { (W-NS) }\end{array}$} & \multirow{6}{*}{ Sample } & $\mathbf{N}$ & 70 & 4 & 1 & & 75 & 63 & 4 & 1 & & 68 \\
\hline & & 85th & 110.9 & & & & 110.9 & 111.2 & & & & 111.3 \\
\hline & & Min. & 79.6 & 91.3 & 93.1 & & 79.6 & 79.6 & 91.3 & 93.1 & & 79.6 \\
\hline & & Max. & 123.0 & 123.6 & 93.1 & & 123.6 & 123.0 & 123.6 & 93.1 & & 123.6 \\
\hline & & Mean & 102.0 & 105.3 & 93.1 & & 102.1 & 102.7 & 105.3 & 93.1 & & 102.7 \\
\hline & & SD & 8.80 & 14.11 & & & 9.05 & 8.93 & 14.11 & & & 9.19 \\
\hline & \multirow{2}{*}{ Population } & 85th & 111.1 & & & & 111.5 & 112.0 & & & & 112.2 \\
\hline & & 95th & 116.5 & & & & 117.0 & 117.4 & & & & 117.8 \\
\hline & & $\mathbf{N}$ & 126 & 4 & 6 & 18 & 153 & 108 & 3 & 5 & 17 & 132 \\
\hline & & 85th & 110.7 & & & 106.8 & 110.7 & 110.9 & & & 106.9 & 110.9 \\
\hline & Sample & Min. & 78.7 & 91.7 & 98.1 & 81.6 & 78.7 & 84.5 & 93.9 & 98.1 & 81.6 & 81.6 \\
\hline Grecnbank Rd & & Max. & 121.4 & 120.2 & 130.8 & 110.8 & 121.4 & 121.4 & 120.2 & 130.8 & 110.8 & 121.4 \\
\hline (E-NS) & & Mean & 101.2 & 101.1 & 112.2 & 98.0 & 101.1 & 102.0 & 104.2 & 112.7 & 98.8 & 101.9 \\
\hline & & SD & 8.14 & 13.06 & 10.61 & 8.43 & 8.35 & 7.93 & 14.06 & 11.80 & 7.92 & 8.11 \\
\hline & Population & 85th & 109.7 & & & 107.0 & 109.7 & 110.3 & & & 107.3 & 110.3 \\
\hline & Topatalion & 95th & 114.6 & & & 112.7 & 114.8 & 115.1 & & & 112.6 & 115.2 \\
\hline & & $\mathbf{N}$ & 99 & 28 & 1 & & 128 & 88 & 28 & 1 & & 117 \\
\hline & & 85th & 109.2 & 117.4 & & & 110.0 & 109.8 & 117.4 & & & 110.3 \\
\hline & Samnle & Min. & 86.8 & 87.5 & 112.9 & & 86.8 & 86.8 & 87.5 & 112.9 & & 86.8 \\
\hline Eagleson Rd & & Max. & 119.0 & 122.0 & 112.9 & & 122.0 & 119.0 & 122.0 & 112.9 & & 122.0 \\
\hline (W-NS) & & Mean & 102.5 & 103.3 & 112.9 & & 102.8 & 102.7 & 103.3 & 112.9 & & 102.9 \\
\hline & & SD & 7.19 & 9.26 & & & 7.69 & 7.51 & 9.26 & & & 7.95 \\
\hline & Ponulation & 85th & 110.0 & 113.1 & & & 110.7 & 110.5 & 113.1 & & & 111.1 \\
\hline & ropatation & 95th & 114.3 & 119.0 & & & 115.4 & 115.0 & 119.0 & & & 116.0 \\
\hline
\end{tabular}




\section{APPENDIX D: SUMMARY OF ADDITIONAL SIGNIFICANT MODELS FOR COMBINED SPEED CHANGE LANES}


Table D-1: Additional Diverge Speed Predictive Models for Combined SCLS

\begin{tabular}{|c|c|c|c|c|c|c|}
\hline Model & Independent Variables & Coefficients & $p$-value & $t$ & $d f$ & $R^{2}$ \\
\hline \multirow{3}{*}{1} & Constant & -63.587 & 0.023 & -2.673 & \multirow{3}{*}{10} & \multirow{3}{*}{0.891} \\
\hline & $V_{R L}$ & 1.487 & 0.000 & 6.810 & & \\
\hline & $L_{D e c}$ & 0.012 & 0.001 & 4.482 & & \\
\hline \multirow{4}{*}{2} & Constant & -121.056 & 0.001 & -4.976 & \multirow{4}{*}{9} & \multirow{4}{*}{0.921} \\
\hline & $V_{R L}$ & 2.050 & 0.000 & 9.854 & & \\
\hline & $Q_{\text {Diverging }}$ & -0.020 & 0.007 & -3.462 & & \\
\hline & $\operatorname{Ramp}_{N L}$ & 7.046 & 0.032 & 2.539 & & \\
\hline \multirow{3}{*}{3} & Constant & 88.713 & 0.000 & 17.705 & \multirow{3}{*}{10} & \multirow{3}{*}{0.640} \\
\hline & $L_{D e c}$ & 0.014 & 0.016 & 2.899 & & \\
\hline & $L_{t}$ & 0.155 & 0.024 & 2.667 & & \\
\hline
\end{tabular}

Table D-2: Additional Speed Differential Predictive Models for Combined SCLs

\begin{tabular}{|c|c|c|c|c|c|c|}
\hline Model & Independent Variables & Coefficients & $p$-value & $t$ & $d f$ & $R^{2}$ \\
\hline \multirow{3}{*}{1} & Constant & 20.019 & 0.000 & 10.538 & \multirow{3}{*}{10} & \multirow{3}{*}{0.921} \\
\hline & $L_{D e c}$ & -0.014 & 0.000 & -9.370 & & \\
\hline & $H V_{R L}$ & -0.864 & 0.000 & -6.095 & & \\
\hline \multirow{3}{*}{2} & Constant & 19.304 & 0.000 & 9.186 & \multirow{3}{*}{10} & \multirow{3}{*}{0.898} \\
\hline & $L_{D e c}$ & -0.014 & 0.000 & -8.244 & & \\
\hline & $H V_{R L-A \text { verage }}$ & -0.725 & 0.000 & -5.160 & & \\
\hline \multirow{3}{*}{3} & Constant & 15.574 & 0.000 & 6.156 & \multirow{3}{*}{10} & \multirow{3}{*}{0.791} \\
\hline & $L_{D e c}$ & -0.013 & 0.000 & -5.071 & & \\
\hline & $L_{t}$ & -0.082 & 0.019 & -2.798 & & \\
\hline \multirow{3}{*}{4} & Constant & 32.649 & 0.001 & 4.530 & \multirow{3}{*}{10} & \multirow{3}{*}{0.901} \\
\hline & $\log L_{D e c}$ & -14.538 & 0.000 & -7.079 & & \\
\hline & $F w y_{N L}$ & 3.029 & 0.013 & 30.17 & & \\
\hline
\end{tabular}

Table D-3: Additional Gore Speed Predictive Model for Combined SCLS

\begin{tabular}{|c|c|c|c|c|c|c|}
\hline Model & Independent Variables & Coefficients & $p$-value & $t$ & $d f$ & $R^{2}$ \\
\hline \multirow{3}{*}{1} & Constant & 31.086 & 0.056 & 2.192 & & \multirow{3}{*}{0.867} \\
\cline { 2 - 6 } & $V_{\text {Diverge }}$ & 0.519 & 0.006 & 3.563 & \multirow{2}{*}{0.867} \\
\cline { 2 - 6 } & $L_{t}$ & 0.146 & 0.009 & 3.338 & & \\
\hline
\end{tabular}


Table D-4: Additional Overall Deceleration Rate Predictive Models for Combined SCLs

\begin{tabular}{|c|c|c|c|c|c|c|}
\hline Model & Independent Variables & Coefficients & $p$-value & $t$ & $d f$ & $R^{2}$ \\
\hline \multirow{3}{*}{1} & Constant & -0.192 & 0.353 & -0.979 & \multirow{3}{*}{9} & \multirow{3}{*}{0.782} \\
\hline & $F w y_{N L}$ & 0.329 & 0.001 & 5.113 & & \\
\hline & $S C L_{\text {Type }}$ & -0.240 & 0.047 & -2.304 & & \\
\hline \multirow{3}{*}{2} & Constant & 0.135 & 0.628 & 0.501 & \multirow{3}{*}{9} & \multirow{3}{*}{0.782} \\
\hline & $F w y_{N L}$ & 0.442 & 0.000 & 5.552 & & \\
\hline & $\operatorname{Ramp}_{W}$ & -0.128 & 0.047 & -2.302 & & \\
\hline \multirow{2}{*}{3} & Constant & 1.336 & 0.000 & 7.691 & \multirow{2}{*}{10} & \multirow{2}{*}{0.648} \\
\hline & $H V_{R L \text {-Average }}$ & -0.058 & 0.002 & -4.288 & & \\
\hline \multirow{2}{*}{4} & Constant & 2.026 & 0.008 & 3.312 & \multirow{2}{*}{10} & \multirow{2}{*}{0.343} \\
\hline & $\log L_{D e c}$ & -0.529 & 0.045 & -2.87 & & \\
\hline
\end{tabular}


APPENDIX E: SUMMARY OF ADDITIONAL SIGNIFICANT MODELS FOR LIMITED LENGTH TYPE SPEED CHANGE LANES 
Table E-1: Additional Diverge Speed Predictive Models for Limited Length SCLS

\begin{tabular}{|c|c|c|c|c|c|c|}
\hline Model & Independent Variables & Coefficients & p-value & $t$ & $d f$ & $R^{2}$ \\
\hline \multirow{3}{*}{1} & Constant & -40.032 & 0.127 & -1.924 & \multirow{3}{*}{4} & \multirow{3}{*}{0.990} \\
\hline & $V_{R L}$ & 1.247 & 0.003 & 6.274 & & \\
\hline & $S C L_{\text {Conf }}$ & 10.414 & 0.015 & 4.121 & & \\
\hline \multirow{3}{*}{2} & Constant & 114.213 & 0.000 & 24.381 & \multirow{3}{*}{4} & \multirow{3}{*}{0.987} \\
\hline & $\theta$ & -1.745 & 0.006 & -5.242 & & \\
\hline & $S C L_{\text {Conf }}$ & 10.563 & 0.024 & 3.538 & & \\
\hline
\end{tabular}

Table E-2: Additional Speed Differential Predictive Model for Limited Length SCLs

\begin{tabular}{|c|c|c|c|c|c|c|}
\hline Model & Independent Variables & Coefficients & $p$-value & $t$ & $d f$ & $R^{2}$ \\
\hline \multirow{3}{*}{1} & Constant & 27.634 & 0.000 & 11.220 & \multirow{3}{*}{4} & \multirow{3}{*}{0.967} \\
\hline & $L_{\text {Lim }}$ & -0.041 & 0.001 & -8.914 & & \\
\hline & $H V_{R L-A v e r a g e}$ & -0.011 & 0.017 & -3.929 & & \\
\hline
\end{tabular}

Table E-3: Additional Gore Speed Predictive Models for Limited Length SCLs

\begin{tabular}{|c|c|c|c|c|c|c|}
\hline Model & Independent Variables & Coefficients & $p$-value & $t$ & $d f$ & $R^{2}$ \\
\hline \multirow{3}{*}{1} & Constant & 1.007 & 0.920 & 0.107 & \multirow{3}{*}{4} & \multirow{3}{*}{0.973} \\
\hline & $V_{\text {Diverge }}$ & 0.986 & 0.000 & 11.456 & & \\
\hline & $Q_{\text {Ramp }}$ & -0.020 & 0.025 & -3.503 & & \\
\hline \multirow{3}{*}{2} & Constant & 135.079 & 0.000 & 29.180 & \multirow{3}{*}{4} & \multirow{3}{*}{0.959} \\
\hline & $\theta$ & -2.832 & 0.001 & -9.264 & & \\
\hline & $Q_{\text {Ramp }}$ & -0.033 & 0.010 & -4.593 & & \\
\hline
\end{tabular}

